AL. $2.1987-14 S_{\text {L.d }}$

$\$ 5.00$

\title{
Synopsis of the
}

Parashes of
Vertebrates
of Canada

Eotoparasites of

Terrestrial Mamnals

Murray J. Kennedy

Robert A. Newman 
Copies of this publication are available from the Queen's Printer, 11510 Kingsway Avenue, Edmonton T5G 2Y5 Orders must be accompanied by a cheque for $\$ 5.00$ made out to the Provincial Treasurer. 


\section{Synopsis of the}

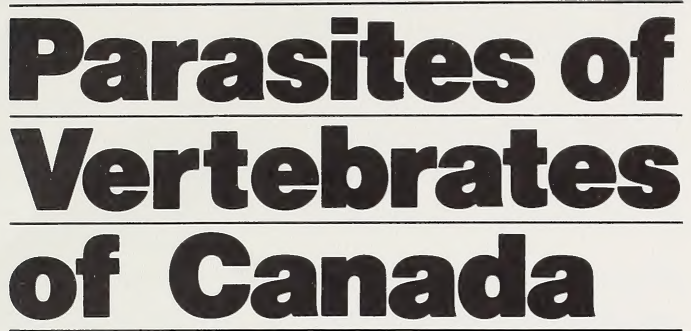

Ectoparasites of

Terrestrial Mammals

Murray J. Kennedy

Robert A. Newman

Alberta Agriculture

Veterinary Laboratory

6909 - 116 Street,

Edmonton, Alberta

Canada

T6H 4P2 
189 A + 477 


\section{Abstract}

This synopsis contains Parasite-Host and Host-Parasite lists of 323 species of ectoparasites from 122 species of terrestrial mammals. The 323 species of ectoparasites are distributed among higher taxa as follows: Acari - 122; Coleoptera - 4; Diptera - 19; Hemiptera - 1; Mallophaga - 19; Siphonaptera - 134; and Siphunculata - 23. 
Digitized by the Internet Archive in 2015 


\section{PARASITE-HOST LIST}




\section{Introduction}

The compiled information covers the period from 1876 to December, 1985 , and was extracted from numerous publications in the primary scientific literature. Journal series such as The Canadian Entomologist, Canadian Journal of Zoology, Journal of Wildlife Diseases and the Journal of Parasitology were searched in their entirety. Abstracting series such as Zoological Record and Entomology Abstracts were scanned for additional references. Theses and reports of federal and provincial governments, and of other institutions, were not searched extensively, hence some existing records may have been missed.

In providing references to the parasite-host-distribution data, we included only reports based on original records, but screening all reports to ensure complete exclusion of repetitive records based on the same material was not possible. Publications that do not provide original parasite-hostdistribution records but deal with some aspect of Canadian ectoparasites are compiled in a supplementary list of references. References dealing only with experimental infections, or with specimens collected from the ground, nests or burrows, or from dead carcasses are generally not included. If they were, a note was added in the remarks section.

The synopsis indicates that up to December, 1985, at least 322 species of ectoparasites have been reported from 122 species of terrestrial mammals. Parasites not identified to the species level are not included in the total number. The 322 species are divided among seven major taxa as follows: Acari - 122; Coleoptera - 4; Diptera - 19; Hemiptera - 1; Mallophaga - 19; Siphonaptera - 134; and Siphunculata - 23.

The taxonomic arrangement adopted for each major taxonomic group of ectoparasite included in Canadian records, in most cases, is as follows:

Acari - Krantz 1978
Coleoptera - Arnett 1968
Diptera - Arnett et al. 1980

Mallophaga - Emerson 1972

Siphonaptera - Holland 1984

Siphunculata - Stojanovich and Pratt 1965

All scientific names of North American mammals are given in the form used by Hall (1981). The entries in the synopsis are organized in accordance with the format used by Margolis and Arthur (1979) in their Synopsis of the Parasites of Fishes of Canada.

The Parasite-Host List is organized by higher taxa of ectoparasite in the following order: Acari, Coleoptera, Diptera, Hemiptera, Mallophaga, Siphonaptera, and Siphunculata. Species, genera, and family names are listed in alphabetical order within their respective next higher taxon, which in the case of a family is either a suborder or an order, depending on the group in question and the usefulness of including the suborder or order category.

For each parasite species listed, the following information is provided:

1) The current scientific name, including author(s) and date(s), followed by any recognized synonyms that were used in establishing the Canadian record(s). No attempt was made to evaluate systematically the validity of pub- lished records, but attention is drawn to obvious errors.

2) The site of occurrence of the ectoparasite on its host(s) is assumed to be the skin for the Acari, Coleoptera, Hemiptera, Mallophaga, Siphonaptera, and Siphunculata. The larval stages of the Diptera are usually found in subcutaneous tissues or air passages of their hosts. Comments on additional sites, or aberrant sites are given in the remarks section.

3) The hosts. Numbers in parentheses after each host name correspond with the numbers assigned to the references establishing the particular parasite-host records. Holland (1984) corrected some host identifications for the Siphonaptera made by previous authors. For the most part we have used Holland's corrections. For example, Wagner (1936a) recorded Foxella ignota recula from Mustela suturata. Holland's correction for this host is Mustela frenata nevadensis.

4) Distribution ( Dist.) Species distribution is indicated by provincial or territory boundaries listed in alphabetical order as follows: Alta (Alberta), BC (British Columbia), Man(Manitoba), NB(New Brunswick), Nfld (Newfoundland), NS (Nova Scotia), NWT (Northwest Territories), Ont (Ontario), PEI (Prince Edward Island), Que (Quebec), Sask (Saskatchewan), and YT (Yukon Territory).

5) Authors responsible for the published records are listed in chronological order. To enable the reader to grasp at a glance the author(s) responsible for particular parasitehost-locality records, we numbered the references listed under "Records" and placed the corresponding number(s) after the host name, and each reference is followed by the locality or localities from which the parasite was (were) reported. When only one host is listed for a particular parasite, the references are not numbered, and when all records are from the same locality, the latter was not listed after the authors' names.

6) Under remarks, explanatory comments, as required, are offered on systematics, nomenclature, misidentifications, and synonyms of parasites; and on the hosts, sites on or within hosts, and validity of previous records.

In the Host-Parasite List, within each order, the families, genera, and species are listed alphabetically. After the name of each parasite species, its geographic distribution on the host in question is given in parentheses.

The REFERENCES are given in two parts, the first includes those directly referred to in the text, and the second, SUPPLEMENTARY REFERENCES, contains a list of publications dealing with various aspects of parasites of wild mammals of Canada but which do not contain original parasite-host records.

To facilitate access to information in the parasite-host and host-parasite lists, the publication concludes with an INDEX to parasite and host scientific names.

Obviously a book of this sort is bound to have errors and ambiguities, and certainly such faults are our responsibility. We hope readers will bring them to our attention. We also hope that colleagues will send us reprints of their current work or papers we missed so that these additions or correc- 
tions can be included in future revisions of the synopsis.

In a sense, this book constitutes a plea and encouragement for more work on our poorly known parasite fauna. We hope this synopsis will kindle interest and raise questions in the minds of future investigators in parasitology. 


\section{PHYLUM CHELICERATA}

CLASS ARACHNIDA

SUBCLASS ACARI

\section{ORDER ACARIDIDA}

\section{Family GLYCYPHAGIDAE}

Dermacarus hylandi Fain, 1969

Syn.: Radfordia hylandi

Hosts: Microtus chrotorrhinus

$M$. pennsylvanicus

Synaptomys borealis

S. cooperi

Tamias striatus

Dist.: NB

Record: Whitaker and French 1982

Dermacarus hypudaei (Koch, 1941)

Host: Napaeozapus insignis

Dist.: NS

Record: Wright 1979

Dermacarus hypudaei septentrionalis (Koch, 1941)

Hosts: Clethrionomys gapperi

Tamiasciurus hudsonicus

Dist.: Que

Record: Gyorkos and Hilton 1982

Dermacarus jonesi Fain and Whitaker, 1976

Host: Peromyscus maniculatus

Dist.: BC

Record: Fain and Whitaker 1976

Dermacarus newyorkensis Fain, 1969

Hosts: Napaeozapus insignis $(2,3)$

Tamiasciurus hudsonicus (1)

Zapus hudsonius $(2,3)$

Dist.: NB, NS, PEI, Que

Records: 1. Gyorkos and Hilton 1982 (Que); 2.

Jones and Thomas 1982 (NB, NS, PEI); 3.

Whitaker and French 1982 (NB)

Dermacarus ondatrae Rupes and Whitaker, 1968

Host: Ondatra zibethicus

Dist.: Man, NS

Records: McKenzie and Welch 1979 (Man);

Wright 1979 (NS)

Dermacarus sciurinus (Koch)

Host: Tamiasciurus hudsonicus

Dist.: Que

Record: Gyorkos and Hilton 1982

Dermacarus validus (Banks)

Host: Ondatra zibethicus osoyoosensis

Dist.: BC

Record: Knight 1951

Dermacarus sp.

Host: Peromyscus maniculatus

Dist.: NB

Record: Whitaker and French 1982
Glycyphagus hypudaei (Koch, 1941)

Hosts: Clethrionomys gapperi

Condylura cristata

Microtus chrotorrhinus

M. pennsylvanicus

Napaeozapus insignis

Peromyscus maniculatus

Sorex fumeus

S. gaspensis

S. palustris

Synaptomys borealis

Dist.: NB

S. cooperi

Record: Whitaker and French 1982

Orycteroxenus canadensis Fain, Kok, Lukoschus and

Hosts: Condylura cristata Clulow, 1971

Dist.: NB

Microsorex hoyi

Microtus chrotorrhinus

Record: Whitaker and French 1982

Orycteroxenus soricis Oudemans, 1915

Hosts: Blarina brevicauda (2)

Condylura cristata (2)

Microsorex hoyi (2)

Microtus chrotorrhinus (2)

Sorex cinereus (2)

$S$. fumeus (2)

$S$. gaspensis (2)

S. palustris (2)

Synaptomys borealis (2)

Zapus hudsonius (1)

Dist.: NB, NS

Records: 1. Jones and Thomas 1982 (NS); 2.

Whitaker and French 1982 (NB)

Xenoryctes latiporus Fain and Whitaker, 1979

Hosts: Blarina brevicauda

Dist.: NB

Zapus hudsonius

Record: Whitaker and French 1982

Xenoryctes nudus Fain and Whitaker, 1975

Hosts: Condylura cristata

Dist.: NB

Sorex fumeus

Record: Whitaker and French 1982

Unidentified Glycephagidae

Glycephagidae gen. spp.

Hosts: Clethrionomys gapperi

Microtus pennsylvanicus

Peromyscus maniculatus

Sorex cinereus

S. palustris

Dist.: Ont

Tamiasciuris hudsonicus

Record: Scholten et al. 1962 
Family LISTROPHORIDAE

Listrophorus americanus Radford, 1944

Hosts: Ondatra zibethicus (2)

O. z. osoyoosensis (1)

Dist.: BC, Man

Records: 1. Knight 1951 (BC); 2. McKenzie

and Welch 1979 (Man)

Listrophorus dicrostonyx Fain and Hyland, 1972

Host: Dicrostonyx sp.

Dist.: NWT

Record: Fain and Hyland 1974

Listrophorus leuckarti Pagenstecher, 1861

Host: Microtus p. pennsylvanicus

Dist.: Ont

Record: McDaniel et al. 1967

Listrophorus mexicanus Fain, 1970

Hosts: Clethrionomys gapperi $(1,3)$

Microtus chrotorrhinus (3)

M. pennsylvanicus (3)

Napaeozapus insignis (2)

Synaptomys cooperi (3)

Zapus hudsonius (2)

Dist.: NB, NS, PEI, Que

Records: 1. Gyorkos and Hilton 1982 (Que); 2.

Jones and Thomas 1982 (NS, PEI, Que); 3.

Whitaker and French 1982 (NB)

Listrophorus mexicanus squamiferus Fain and

Host: Microtus pennsylvanicus

Hyland, 1972

Dist.: NWT

Record: Fain and Hyland 1974

Listrophorus ondatrae Fain, Kok, and Lukoschus, 1970

Host: Ondatra zibethicus

Dist.: NWT

Record: Fain 1970

Listrophorus phenacomys Fain and Hyland, 1972

Hosts: Phenacomys intermedius

Phenacomys sp.

Dist.: Man, NWT

Record: Fain and Hyland 1974

Listrophorus synaptomys (Fain, Whitaker

Hosts: Synaptomys borealis McDaniel, and Lukoschus)

Dist.: NB

$$
\text { S. cooperi }
$$

Record: Whitaker and French 1982

Listrophorus validus Banks, 1909

Host: Ondatra zibethicus

Dist.: Ont

Records: Banks 1909; Jarvis 1910

Listrophorus spp.

Host: Blarina brevicauda

Dist.: Ont

Record: Jameson 1950
Lynxacarus mustelae (Megnin, 1885) Fain and Hyland, 1974

Syn.: Listrophorus mustelae Megnin, 1885

Host: Martes americana abietinoides

Dist.: BC

Record: Cowan 1955

Prolabidocarpus canadensis Lawrence, 1948

Host: Castor canadensis

Dist.: Ont

Record: Lawrence 1948

Unidentified Listrophoridae

Listrophoridae gen. spp.

Hosts: Clethrionomys gapperi

Dist.: Ont Microtus pennsylvanicus

Record: Scholten et al. 1962

Family MYOCOPTIDAE

Gliricoptes zapus Fain and Whitaker, 1974

Host: Napaeozapus insignis

Dist.: NB

Record: Whitaker and French 1982

Gliricoptes spp.

Hosts: Napaeozapus insignis

Dist.: Que Tamiasciurus hudsonicus

Record: Gyorkos and Hilton 1982

Remarks: Gyorkos and Hilton's (1982)

specimens were near $G$. glirinus (Canestrini).

Myocoptes japonensis canadensis Radford, 1955

Syn.: Myocoptes canadensis Radford, 1955

Host: Dicrostonyx spp.

Dist.: Ont

Record: Radford 1955

Myocoptes japonensis japonensis Radford, 1955

Hosts: Clethrionomys gapperi

Microtus chrotorrhinus

Dist.: NB M. pennsylvanicus

Record: Whitaker and French 1982

Myocoptes squamosus Fain, Munting, and Lukoschus, 1969

Hosts: Clethrionomys gapperi

Microtus chrotorrhinus

Dist.: NB M. pennsylvanicus

Record: Whitaker and French 1982

Myocoptes spp.

Hosts: Blarina brevicauda (1)

Clethrionomys gapperi (3)

C. rutilus (2)

Peromyscus maniculatus (3)

Tamias striatus (3)

Dist.: NWT, Ont, Que

Records: 1. Jameson 1950 (Ont); 2. Mitchell and Behin 1965 (NWT); 3. Gyorkos and Hilton 1982 (Que) 
Remarks: Gyorkos and Hilton (1982) noted that their specimens from $P$. maniculatus were near Myocoptes musculinus; those from $C$. gapperi were near $M$. ondatrae; and those from $T$. striatus, and some from C. gapperi, were immature.

Trichoecius tenax (Michael, 1889) Fain, Munting, and Lukoschus, 1969

Syn.: Myocoptes tenax Michael, 1889

Hosts: Clethrionomys gapperi

Microtus chrotorrhinus

Dist.: NB

M. pennsylvanicus

Record: Whitaker and French 1982

Trichoecius spp.

Hosts: Microtus chrotorrhinus Peromyscus maniculatus

Dist.: NB

Record: Whitaker and French 1982

Family PSOROPTIDAE

Chorioptes texanus Hirst, 1924

Host: Rangifer tarandus

Dist.: NWT

Record: Sweatman 1958a

Otodectes cynotis (Hering, 1838) Canestrini, 1894

Hosts: Odocoileus virginianus Vulpes vulpes

Dist.: Alta, Que

Record: Sweatman 1958b

Remarks: The mites were recovered from the ear canal.

Psoroptes ovis (Hering, 1838)

Host: Ovis canadensis

Dist.: Alta

Record: Cowan 1944

\section{Family SARCOPTIDAE}

Sarcoptes scabiei (Linnaeus, 1758)

Hosts: Canis latrans

C. lupus

Vulpes vulpes

Dist.: Alta

Record: Samuel 198

Unidentified Sarcoptidae

Sarcoptidae gen. spp.

Hosts: Clethrionomys gapperi

Dist.: Ont Tamias striatus

Record: Scholten et al. 1962

\section{SUBORDER ACTINEDIDA} Family CHEYLETIDAE

Cheyletiella parasitivorax (Megnin, 1878)

Host: Lepus americanus

Dist.: Ont

Record: Cooper 1946
Family MYOBIIDAE

Amorphacarus hengererorum Jameson, 1948

Hosts: Blarina brevicauda

Microsorex hoyi

Sorex cinereus

S. fumeus

S. gaspensis

Dist.: NB

S. palustris

Record: Whitaker and French 1982

Blarinobia simplex (Ewing, 1938)

Syn.: Myobia simplex Ewing, 1938

Host: Blarina brevicauda

Dist.: NB, Ont

Records: 1. Jameson 1948 (Ont), 2. 1950 (Ont);

3. Whitaker and French 1982 (NB)

Eadiea condylurae Jameson, 1949

Host: Condylura cristata

Dist.: NB

Record: Whitaker and French 1982

Myobia caudata Banks, 1909

Host: Little brown bat

Dist.: Ont

Record: 1. Banks 1909; 2. Jarvis 1910;

Myobia musculi (Schrank, 1781)

Host: House mouse

Dist.: Ont

Record: Jarvis 1910

Myobia spp.

Hosts: Clethrionomys gapperi (3)

Microtus pennsylvanicus (3)

Napaeozapus insignis (3)

Peromyscus maniculatus (3)

Peromyscus spp. (1)

Sorex cinereus (2)

Dist.: BC, NS, Que

Records: 1. Spencer 1940 (BC); 2. Wright 1979

(NS); 3. Gyorkos and Hilton 1982 (Que)

Protomyobia americana McDaniel, 1967

Hosts: Blarina brevicauda

Dist.: NB Microsorex hovi

Record: Whitaker and French 1982

Protomyobia brevisetosa Jameson, 1948

Hosts: Microtus pennsylvanicus Sorex fumeus

Dist.: NB

Record: Whitaker and French 1982

Protomyobia claparedei (Poppe, 1896)

Hosts: Blarina brevicauda $(1,2)$

Sorex cinereus (3)

$S$. palustris (3)

Dist.: NB, Ont

Records: 1. Jameson 1948 (Ont), 2. 1950 (Ont);

3. Whitaker and French 1982 (NB) 
Radfordia ewingi (Fox, 1937)

Hosts: Napaeozapus insignis $(1,2)$

Zapus hudsonius (1)

Dist.: NB, PEI

Records: 1. Jones and Thomas 1982 (NB, PEI);

2. Whitaker and French 1982 (NB)

Radfordia lemnina (Koch, 1841)

Host: Clethrionomys gapperi

Dist.: NB

Record: Whitaker and French 1982

Radfordia subuliger Ewing, 1938

Hosts: Peromyscus maniculatus (2)

P. m. austerus (1)

Dist.: BC, NB

Records: 1. Spencer 1940 (BC); 2. Whitaker

and French 1982 (NB)

Radfordia spp.

Hosts: Clethrionomys gapperi (3)

Microtus chrotorrhinus (4)

M. pennsylvanicus (3)

Ondatra zibethicus osoyoosensis (2)

Peromyscus maniculatus (3)

Rattus norvegicus (1)

Dist.: BC, NB, Que

Records: 1. Firlotte 1948 (Que); 2. Knight 1951

(BC); 3. Gyorkos and Hilton 1982 (Que); 4.

Whitaker and French 1982 (NB)

Remarks: Knight's (1951) specimens closely resembled $R$. lemnina. The Radfordia sp. from

C. gapperi and $M$. pennsylvanicus was close to

$R$. lemnina; those from $P$. maniculatus were

immature (Gyorkos and Hilton 1982).

Myobiidae gen. spp.

Unidentified Myobiidae

Hosts: Blarina brevicauda

Clethrionomys gapperi

Microtus pennsylvanicus

Mus musculus

Peromyscus maniculatus

Sorex cinereus

Zapus hudsonius

Dist.: Ont

Record: Scholten et al. 1962

\section{Family PSORERGATIDAE}

Psorergates canadensis Kok, Lukoschus, and Clulow, 1971

Host: Microtus pennsylvanicus

Dist.: Man, Ont

Record: Kok et al. 1971

Remarks: The specimens were found only in the ear concha and on the hind legs.

Psorergates cinereus Kok, Lukoschus, and Clulow, 1971

Host: Sorex cinereus

Dist.: Man, Ont

Record: Kok et al. 1971
Remarks: The specimens were found only in the ear concha and on the hind legs.

Psorergates simplex Tyrrell, 1883

Host: Mouse

Dist.: unspecified

Record: Tyrrell 1883

Remarks: Found in the ear.

Psorergates watsoni Kok, Lukoschus, and Clulow, 1971

Host: Peromyscus maniculatus

Dist.: Ont

Record: Kok et al. 1971

Remarks: The specimens were found only in the ear concha and on the hind legs.

Psorergates spp.

Hosts: Field mouse

House mouse

Dist.: Ont

Record: Jarvis 1910

Psorobia castoris Kok, Lukoschus, and Clulow, 1970

Host: Castor canadensis

Dist.: Ont

Record: Kok et al. 1970

Family PYGMEPHORIDAE

Bakerdania spp.

Hosts: Blarina brevicauda

Microsorex hoyi

Microtus chrotorrhinus

Peromyscus maniculatus

Sorex cinereus

S. fumeus

S. gaspensis

Dist.: NB

Synaptomys cooperi

Record: Whitaker and French 1982

Pygmephorus horridus Mahunka, 1973

Hosts: Blarina brevicauda

Clethrionomys gapperi

Condylura cristata

Microsorex hoyi

Microtus chrotorrhinus

Napaeozapus insignis

Sorex cinereus

S. fumeus

S. gaspensis

S. palustris

Synaptomys borealis

Dist.: NB

S. cooperi

Record: Whitaker and French 1982

Pygmephorus johnstoni Smiley and Whitaker, 1979

Host: Blarina brevicauda

Dist.: NB

Record: Whitaker and French 1982 
Pygmephorus lutterloughae Smiley and Whitaker, 1979

Hosts: Blarina brevicauda

Dist.: NB Condylura cristata

Record: Whitaker and French 1982

Pygmephorus mahunkai Smiley and Whitaker, 1979

Hosts: Myotis lucifugus (2)

Napaeozapus insignis (1)

Zapus hudsonius (1)

Dist.: NS, PEI

Records: 1. Jones and Thomas 1982 (NS, PEI), 2. 1983 (PEI)

Pygmephorus moreohorridus Mahunka, 1975

Hosts: Blarina brevicauda

Condylura cristata

Microsorex hoyi

Microtus pennsylvanicus

Sorex cinereus

S. fumeus

S. gaspensis

S. palustris

Dist.: NB

Record: Whitaker and French 1982

Pygmephorus nidicolus Mahunka, 1969

Hosts: Blarina brevicauda

Dist.: NB

Sorex gaspensis

Record: Whitaker and French 1982

Pygmephorus whitaker, Mahunka, 1973

Hosts: Blarina brevicauda

Sorex cinereus

S. fumeus

Dist.: NB

S. gaspensis

Record: Whitaker and French 1982

Pygmephorus spp.

Hosts: Blarina brevicauda (2)

Dicrostonyx groenlandicus (1)

Sorex fumeus (2)

Dist.: NB, NWT

Records: 1. McAlpine 1965 (NWT); 2.

Whitaker and French 1982 (NB)

Family TROMBICULIDAE

Comatacarus americanus Ewing, 1942

Host: Blarina brevicauda

Dist.: Ont

Record: Jameson 1950

Euschoengastia blarinae (Ewing, 1931)

Hosts: Blarina brevicauda (1)

Clethrionomys gapperi (2)

Microtus chrotorrhinus (2)

Sorex cinereus (1)

S. fumeus (1)

Dist.: NB, Ont

Record: 1. Jameson 1950 (Ont); 2. Whitaker and French 1982 (NB)
Euschoengastia setosa (Ewing, 1937)

Hosts: Microtus chrotorrhinus (2)

Peromyscus maniculatus (2)

Tamias striatus (1)

Dist.: NB, Que

Record: 1. Gyorkos and Hilton 1982 (Que); 2.

Whitaker and French 1982 (NB)

Euschoengastia spp.

Hosts: Clethrionomys gapperi (2) Microtus pennsylvanicus $(1,2)$

Dist.: NS, Sask

Records: 1. Wilson 1967 (Sask); 2. Wright 1979 (NS)

Remarks: Wilson (1967) stated that his material was close to E. campi Brown and Brennan and E. sciuricola (Ewing).

Eutrombicula harperi Ewing, 1928

Host: Ondatra zibethicus osoyoosensis

Dist.: BC

Record: Knight 1951

Miyatrombicula esoensis Sasa and Ogata, 1953

Hosts: Blarina brevicauda (2)

Clethrionomys gapperi (2)

Microsorex hoyi (2)

Microtus chrotorrhinus (2)

M. pennsylvanicus (2)

Napaeozapus insignis $(1,2)$

Peromyscus maniculatus (2)

Sorex cinereus (2)

S. fumeus (2)

S. gaspensis (2)

S. palustris (2)

Synaptomys borealis (2)

S. cooperi (2)

Tamias striatus (2)

Dist.: NB, PEI

Records: 1. Jones and Thomas 1982 (PEI); 2.

Whitaker and French 1982 (NB)

Neotrombicula basignata (Hirst, 1925)

Hosts: Lepus americanus

Dist.: NS

Microtus pennsylvanicus

Record: Wright 1979

Neotrombicula harperi (Ewing, 1928)

Hosts: Clethrionomys gapperi (2)

Microtus chrotorrhinus (2)

M. pennsylvanicus (2)

Napaeozapus insignis $(1,2)$

Sorex cinereus (2)

Synaptomys cooperi (2)

Tamias striatus (2)

Zapus hudsonius (1)

Dist.: NB, NS, PEI, Que

Records: 1. Jones and Thomas 1982 (NB, NS,

PEI, Que); 2. Whitaker and French 1982 (NB)

Neotrombicula lipovskyi Brennan and Wharton, 1950

Host: Zapus hudsonius

Dist.: NB

Record: Jones and Thomas 1982 
Neotrombicula microti (Ewing, 1928)

Hosts: Blarina brevicauda (1)

Clethrionomys gapperi $(1,3)$

Microtus pennsylvanicus (1)

Napaeozapus insignis $(1,2)$

Ondatra zibethicus (1)

Peromyscus leucopus (1)

Tamiasciurus hudsonicus (1)

Zapus hudsonius (1)

Dist.: NB, NS, Que

Records: 1. Wright 1979 (NS); 2. Jones and

Thomas 1982 (NS, Que); 3. Whitaker and

French 1982 (NB)

Trombicula jamesoni Brennan, 1948

Host: Blarina brevicauda

Dist.: Ont

Record: Jameson 1950

Trombicula spp.

Hosts: Clethrionomys gapperi $(2,3)$

Peromyscus leucopus (2)

$P$. maniculatus austerus (1)

Tamiasciurus hudsonicus (3)

Dist.: BC, NS, Que

Records: 1. Spencer 1940 (BC); 2. Wright 1979

(NS); 3. Gyorkos and Hilton 1982 (Que)

Unidentified Trombiculidae

Trombiculidae gen. spp.

Hosts: Clethrionomys gapperi

Odocoileus virginianus

Peromyscus maniculatus

Tamiasciurus hudsonicus

Tamias striatus

Zapus hudsonius

Dist.: Ont

Record: Scholten et al. 1962

\section{Family TROMBIDIIDAE}

Allothrombium spp.

Host: Microtus pennsylvanicus

Dist.: NS

Record: Wright 1979

Neoschongastia blarinae (Ewing, 1929)

Host: Streator's red squirrel

Dist.: BC

Record: Spencer 1940

ORDER PARASITIFORMES

\section{SUBORDER GAMASIDA}

\section{Family DERMANYSSIDAE}

Liponyssus montanus (Ewing, 1923)

Hosts: Spermophilus richardsonii

Dist.: Man

$$
\text { S. tridecemlineatus }
$$

Record: McLeod 1933
Liponyssus occidentalis (Ewing, 1923)

Syn.: Ceratonyssus occidentalis (Ewing, 1923)

Hosts: Myotis l. lucifugus (2)

Spermophilus richardsonii (1)

S. tridecemlineatus (1)

Bats (3)

Dist.: BC, Man, Ont

Record: 1. McLeod 1933 (Man); 2. Dymond

1938 (Ont); 3. Spencer 1939 (BC)

Liponyssus spp.

Hosts: Condylura cristata (1)

Bats (2)

Streator's red squirrel (3)

Dist.: BC, Ont

Records: 1. Jarvis 1910 (Ont); 2. Spencer 1939a

(BC), 3. 1940 (Ont)

Myonyssus jamesoni Ewing and Baker, 1947

Hosts: Blarina brevicauda $(1,2)$

Sorex fumeus (2)

S. palustris (3)

Dist.: NB, Ont

Records: 1. Ewing and Baker 1947 (Ont); 2.

Jameson 1950 (Ont); 3. Whitaker and French 1982 (NB)

Remarks: Whitaker and French (1982) placed $M$. jamesoni in the family Laelapidae.

Family HALARACHNIDAE

Pneumonyssus spp.

Host: Spermophilus richardsonii

Dist.: Alta

Record: Hilton and Mahrt 1971

Zumptiella bakeri(Furman)

Host: Tamiasciurus hudsonicus

Dist: Que

Record: Gyorkos and Hilton 1982

$$
\text { Family LAELAPIDAE }
$$

Androlaelaps casalis (Berlese, 1887)

Host: Peromyscus maniculatus

Dist.: NB

Record: Whitaker and French 1982

Androlaelaps fahrenholzi (Berlese, 1911)

Syn.: Androlaelaps glasgowi (Ewing, 1925) Atricholaelaps glasgowi (Ewing, 1925)

Hosts: Blarina brevicauda $(2,6)$

Clethrionomys gapperi $(4,6)$

Condylura cristata (6)

Microtus chrotorrhinus (6)

M. pennsylvanicus $(3,6)$

M. p. pennsylvanicus $(1,2)$

$M$. pinetorum scalopsoides (1)

Napaeozapus insignis $(5,6)$

Peromyscus maniculatus (6)

Peromyscus sp. (1)

Rattus norvegicus (1)

Sorex fumeus (6)

Synaptomys borealis (6) 
S. cooperi (6)

Zapus hudsonius (5)

Z. h. ontarioensis (1)

Dist.: NB, NS, Ont, PEI, Sask

Records: 1. Judd 1950 (Ont), 2. 1953 (Ont); 3.

Wilson 1967 (Sask); 4. Wright 1979 (NS); 5.

Jones and Thomas 1982 (NB, NS, PEI); 6.

Whitaker and French 1982 (NB)

Androlaelaps spp.

Hosts: Blarina brevicauda

Clethrionomys gapperi

Marmota monax

Microtus pennsylvanicus

Napaeozapus insignis

Peromyscus leucopus

$P$. maniculatus

Dist.: NS

Zapus hudsonius

Record: Wright 1979

Echinolaelaps echidninus (Berlese, 1887)

Host: Rattus norvegicus

Dist.: Que

Record: Firlotte 1948

Echinonyssus blarinae

Host: Blarina brevicauda

Dist.: NB

Record: Whitaker and French 1982

Echinonyssus isabellinus (Oudemans, 1913)

Hosts: Blarina brevicauda (2)

Clethrionomys gapperi (2)

Condylura cristata (2)

Microtus chrotorrhinus (2)

M. pennsylvanicus (2)

Napaeozapus insignis (1)

Synaptomys cooperi (2)

Zapus hudsonius (1)

Dist.: NB, PEI

Records: 1. Jones and Thomas 1982 (PEI); 2.

Whitaker and French 1982 (NB)

Echinonyssus talpae (Zemskaya, 1955)

Hosts: Blarina brevicauda

Microsorex hoyi

Sorex cinereus

S. fumeus

S. gaspensis

Dist.: NB

S. palustris

Record: Whitaker and French 1982

Echinonyssus spp.

Host: Clethrionomys gapperi

Dist.: NB

Record: Whitaker and French 1982

Eulaelaps stabularis (Koch, 1836)

Syn.: Laelaps pedalis Banks, 1909

Hosts: Blarina brevicauda $(3,6,7)$

Clethrionomys gapperi $(6,7)$

Condylura cristata (7)
Microtus chrotorrhinus (7)

M. pennsylvanicus $(5,6)$

Peromyscus leucopus (6)

P. maniculatus (7)

Peromyscus spp. (4)

Sorex cinereus $(5,6)$

Synaptomys cooperi (7)

Chipmunk $(1,2)$

Dist.: NB, NS, Ont, Sask

Records: 1. Banks 1909 (Ont); 2. Jarvis 1910

(Ont); 3. Jameson 1950 (Ont); 4. Judd 1950

(Ont); 5. Wilson 1967 (Sask); 6. Wright 1979

(NS); 7. Whitaker and French 1982 (NB)

Gemasus spp.

Host: Red squirrel

Dist.: Ont

Record: Jarvis 1910

Haemogamasus ambulans (Thorell, 1872)

Syn.: Haemogamasus alaskensis Ewing, 1925

Hosts: Blarina brevicauda $(1,7)$

Clethrionomys gapperi $(5,7)$

C. rutilus (3)

Condylura cristata (7)

Dicrostonyx groenlandicus (2)

Eutamias minimus (4)

Microsorex hoyi $(7)$

Microtus chrotorrhinus (7)

M. pennsylvanicus (7)

Napaeozapus insignis (6)

Sorex fumeus (7)

S. gaspensis (7)

S. palustris (7)

Synaptomys cooperi (7)

Zapus hudsonius $(6,7)$

Dist.: NB, NS, NWT, Ont, PEI

Records: 1. Jameson 1950 (Ont); 2. McAlpine 1965 (NWT); 3. Mitchell and Behin 1965

(NWT); 4. Wilson 1967 (Ont); 5. Wright 1979

(NS); 6. Jones and Thomas 1982 (NB, PEI); 7.

Whitaker and French 1982 (NB)

Remarks: Mitchell and Behin (1965) have

Haemogamasus alaskensis and $H$. ambulans

listed as seperate species.

Haemogamasus hirsutus Berlese, 1889

Host: Zapus hudsonius ontarioensis

Dist.: Ont

Record: Judd 1950

Haemogamasus liponyssoides Ewing, 1925

Syn.: Euhaemogamasus liponyssoides (Ewing, 1925)

Hosts: Blarina brevicauda $(2,3)$

Condylura cristata (3)

Microtus chrotorrhinus (3)

Scapanus orarius schefferi (1)

Sorex cinereus (3)

S. fumeus (3)

S. gaspensis (3)

Synaptomys cooperi (3) 
Mole (1)

Dist.: BC, NB, Ont

Records: 1. Spencer 1940 (BC); 2. Jameson

1950 (Ont); 3. Whitaker and French 1982 (NB)

Haemogamasus longitarsus (Banks, 1910)

Syn.: Euhaemogamasus microti (Ewing, 1925)

Host: Blarina brevicauda

Dist.: Ont

Record: Jameson 1950

Haemogamasus reidi Ewing, 1925

Host: Clethrionomys gapperi

Dist.: NB

Record: Whitaker and French 1982

Haemogamasus spp.

Hosts: Blarina brevicauda

Clethrionomys gapperi

Condylura cristata

Glaucomys sabrinus

Microtus pennsylvanicus

Napaeozapus insignis

Peromyscus leucopus

Sorex cinereus

Dist.: NS

S. palustris

Record: Wright 1979

Haemolaelaps glasgowi (Ewing, 1925)

Hosts: Blarina brevicauda (1)

Clethrionomys gapperi (2)

Marmota monax (3)

Peromyscus maniculatus (2)

Tamiasciurus hudsonicus (2)

Tamias striatus (2)

Dist.: Ont

Records: 1. Jameson 1950; 2. Scholten et al.

1962; 3. Ko 1972 b

Hirstionyssus isabellinus (Oudemans, 1913)

Hosts: Clethrionomys rutilus (2)

Dist.: NWT

Dicrostonyx groenlandicus (1)

Records: 1. McAlpine 1965; 2. Mitchell and

Behin 1965

Hirstionyssus occidentalis (Ewing)

Host: Spermophilus richardsonii

Dist.: Alta

Record: Hilton and Mahrt 1971

Hirstionyssus talpae Zemskaya, 1955

Host: Blarina brevicauda

Dist.: NS

Record: Wright 1979

Hirstionyssus spp.

Hosts: Blarina brevicauda $(1,4)$

Clethrionomys gapperi (4)

Condylura cristata (4)

Mephitis mephitis $(2,3)$

Microtus pennsylvanicus $(3,4)$

Peromyscus maniculatus $(3,4)$

Procyon lotor (4)
Sorex palustris (3)

Dist.: NS, Ont

Records: 1. Jameson 1950 (Ont); 2. Judd 1953

(Ont); 3. Scholten et al. 1962 (Ont); 4. Wright 1979 (NS)

Hyperlaelaps spp.

Hosts: Clethrionomys gapperi Microtus pennsylvanicus

Dist.: NS Zapus hudsonius

Record: Wright 1979

Hypoaspis spp.

Host: Peromyscus leucopus

Dist.: NS

Record: Wright 1979

Laelaps alaskensis Grant, 1947

Hosts: Dicrostonyx groenlandicus (1)

Microtus chrotorrhinus (3)

M. pennsylvanicus $(2,3)$

Synaptomys borealis (3)

S. cooperi (3)

Dist.: NB, NWT, Ont

Records: 1. McAlpine 1965 (NWT); 2. Wilson

1967 (Ont); 3. Whitaker and French 1982 (NB)

Laelaps clethrionomydis Lange, 1955

Host: Clethrionomys rutilus

Dist.: NWT

Record: Mitchell and Behin 1965

Laelaps kochi Oudemans, 1936

Syn.: Laelaps microti (Ewing, 1933)

Tetragonyssus microti Ewing, 1933

Hosts: Blarina brevicauda (1)

Clethrionomys gapperi (5)

Glaucomys sabrinus (5)

Microtus chrotorrhinus (7)

M. pennsylvanicus $(5,7)$

M. p. pennsylvanicus $(2,3,4)$

Napaeozapus insignis (6)

Synaptomys cooperi (7)

Dist.: NB, NS, Ont, PEI

Records: 1. Jameson 1950 (Ont); 2. Judd 1950

(Ont), 3. 1953 (Ont), 4. 1954a (Ont); 5. Scholten

et al. 1962 (Ont); 6. Jones and Thomas 1982

(NS, PEI); 7. Whitaker and French 1982 (NB)

Laelaps multispinosus Banks, 1909

Syn.: Ondatralaelaps multispinosa (Banks, 1910)

Hosts: Ondatra zibethicus $(1,2,4,5)$

O. z. osoyoosensis (3)

Dist.: BC, Man, NS, Ont

Records: 1. Banks 1909 (Ont); 2. Jarvis 1910

(Ont); 3. Knight 1951 (BC); 4. McKenzie and

Welch 1979 (Man); 5. Wright 1979 (NS)

Laelaps muris (Ljungh, 1799)

Hosts: Microtus p. pennsylvanicus $(1,2,3)$ Ondatra z. zibethicus (1)

Dist.: Ont

Records: 1. Judd 1950, 2. 1953, 3. 1954a 
Remarks: Records of $L$. muris by Judd (1950, $1953,1954 a)$ probably belong to $L$. alaskensis (Wilson 1967).

Laelaps propheticus Banks, 1909

Host: Groundhog

Dist.: Ont

Records: Banks 1909; Jarvis 1910

Laelaps spp.

Hosts: Clethrionomys gapperi (3) Microtus pennsylvanicus (3) Ondatra zibethicus (1)

Rattus norvegicus (2)

Dist.: BC, NS, Que

Records: 1. Spencer 1940 (BC); 2. Firlotte 1948

(Que); 3. Wright 1979 (NS)

Laelaspis spp.

Host: Zapus hudsonius

Dist.: NS

Record: Wright 1979

Ololaelaps spp.

Hosts: Microtus pennsylvanicus

Dist.: NS Zapus hudsonius

Record: Wright 1979

Family MACRONYSSIDAE

Macronyssus crosbyi (Ewing and Stover, 1915)

Hosts: Clethrionomys gapperi (1) Myotis lucifugus (2) Myotis spp. (1)

Dist.: NS, PEI

Records: 1. Wright 1979 (NS); 2. Jones and

Thomas 1983 (PEI)

Ornithonyssus bacoti (Hirst, 1913)

Syn.: Liponyssus bacoti Hirst, 1913

Hosts: Rattus norvegicus $(1,2,3)$ Rattus rattus (3)

Dist.: BC

Records: 1. Spencer 1937, 2. 1939b, 3. 1940

Steatonyssus occidentalis (Ewing, 1933) Whitaker and Wilson, 1974

Syn.: Caratonyssus occidentalis Ewing, 1933

Hosts: Myotis l. lucifugus (1) Bats (2)

Dist.: BC, Ont

Records: 1. Dymond 1938 (Ont); 2. Spencer 1939a (BC)

Family OLOGAMASIDAE

Cyrtolaelaps spp.

Hosts: Blarina brevicauda Clethrionomys gapperi

Condylura cristata

Microsorex hoyi

Microtus chrotorrhinus

Peromyscus maniculatus
Sorex cinereus

S. fumeus

S. gaspensis

Dist.: NB

S. palustris

Record: Whitaker and French 1982

Euryparasitus spp.

Hosts: Blarina brevicauda Condylura cristata Microtus chrotorrhinus M. pennsylvanicus Napaeozapus insignis Sorex cinereus

S. fumeus

S. gaspensis

Synaptomys borealis

Dist.: NB

S. cooperi

Record: Whitaker and French 1982

Family SPINTURNICIDAE

Spinturnix americanus (Banks, 1902)

Host: Myotis lucifugus

Dist.: PEI

Record: Jones and Thomas 1983

Spinturnix globosus (Rudnick, 1960) Domrow, 1972

Syn.: Paraspinturnix globosus Rudnick, 1960

Host: Myotis lucifugus

Dist.: Alta

Record: Smith 1981

Remarks: The mites were collected in and around the orifice of the bats (Smith 1981).

Spinturnix spp.

Hosts: Eptesicus fuscus (4)

Myotis l. lucifugus (1)

Myotis spp. (5)

Bats $(2,3)$

Dist.: BC, NS, Ont

Records: 1. Dymond 1938 (Ont); 2. Spencer 1939a (BC), 3. 1940 (BC); 4. Judd 1953 (Ont); 5. Wright 1979 (NS)

\section{SUBORDER IXODIDA}

\section{Family ARGASIDAE}

Ornithodoros kelleyi Cooley and Kohls, 1941

Host: Bats

Dist.: Alta, Sask

Record: Wilkinson et al. 1981

Remarks: The specimens were collected from bat-inhabited buildings (Wilkinson et al. 1980).

Ornithodoros spp.

Host: Eumops perotis californicus

Dist.: BC

Record: Spencer 1939a 
Otobius megnini (Duges, 1884) Banks, 1912

Syn.: Ornithodoros megnini Duges, 1884

Hosts: Cervus elaphus (6)

Odocoileus hemionus $(5,6)$

$O$. virginianus (6)

Oreamnos americanus $(3,5,6,7)$

Ovis canadensis $(5,6,7)$

O. c. californiana (8)

Jack rabbit (2)

Rabbit (1)

Dist.: Alta, BC, Man

Records: 1. Hadwen 1913 (Alta); 2. Hewitt

1915 (Alta); 3. Cowan 1944 (Alta); 4. MacNay

1952 (BC); 5. Gregson 1953 (BC); 6. Rich 1957

(BC); 7. MacNay 1958 (BC); 8. Blood 1963

(BC)

Remarks: Brown and Kohls (1950) refer to

Hadwen's (1913) specimens as Otobius

lagophilus Cooley and Kohls.

\section{Family IXODIDAE}

Dermacentor albipictus (Packard, 1869) Banks, 1907

Hosts: Alces alces $(1,2,3,6,7,9,10,12,13,14$, $16,21,22,23,25,26,27)$

Canis latrans $(5,20)$

Cervus elaphus $(2,3,6,14,18)$

Odocoileus hemionus $(3,5,6,24)$

O. h. columbianus (8)

O. h. hemionus $(4,6,20)$

O. virginianus $(21,23,24)$

Oreamnos americanus (17)

Ovis canadensis (6)

O. c. californiana (15)

Rangifer tarandus caribou (19)

Coast deer (5)

Deer $(7,11,12,14)$

Dist.: Alta, BC, Man, NB, NS, Ont, Que, Sask Records: 1. Jarvis 1910 (Ont); 2. Hewitt 1915

(BC, Man, Que); 3. Cameron and Fulton 1927

(Sask); 4. Buckell 1934 (BC); 5. Hearle 1938

(BC); 6. Cowan 1944 (Alta); 7. Bequaert 1945

(NB, NS, Ont); 8. Cowan 1946 (BC); 9. Brown and Kohls 1950 (Alta); 10. Peterson 1955

(Que); 11. Banfield 1956 (Alta); 12. Gregson 1958 (BC); 13. Ritcey and Edwards 1958 (BC); 14. Stelfox 1962 (Alta); 15. Blood 1963 (BC); 16. Fyvie 1966 (Ont); 17. Kerr and Holmes 1966 (Alta); 18. Flook and Stenton 1969 (Alta, BC); 19. Martell et al. 1969 (NS); 20. Wilkinson 1970 (BC); 21. Addison et al. 1979 (Ont); 22.

Samuel and Barker 1979 (Alta); 23. Wright 1979 (NS); 24. Samuel et al. 1980 (Alta); 25.

Timmerman and Lankester 1980 (Ont); 26.

Drew and Samuel 1985 (Alta), 27. 1986 (Alta)

Remarks: Cameron and Fulton (1927) reported Alces machlis and Cervus canadensis as hosts to $D$. albipictus. These hosts are recorded here as Alces alces and Cervus elaphus, respectively. Buckell (1934) presumed the nymphs he collected were D. albipictus.
Dermacentor andersoni Stiles, 1908

Syn.: Dermacentor venustus Marx in Neumann, 1897

Hosts: Alces alces $(12,13,18)$

A. a. andersoni (17)

Antilocapra a. americana (17)

Canis latrans (7)

Cervus elaphus (13)

Erethizon dorsatum nigriscens (17)

E. d. epixanthium (17)

Erethizon sp. (12)

Lepus townsendii campanius (7)

Marmota caligata (17)

M. flaviventris avara $(6,12)$

M. $\operatorname{monax}(17,18)$

Marmota sp. (7)

Mephitis mephitis (17)

Mustela frenata nevadensis (17)

M. putorius (lab animal) (17)

Neotoma c. cinerea (16)

Odocoileus hemionus $(1,5,6,9)$

$O . h$. hemionus $(12,14,17)$

Oreamnos americanus $(4,9,15,19)$

O. a. missoulae (12)

Ovis canadensis $(1,7,8,11)$

O. c. canadensis (12)

Spermophilus columbianus $(5,11)$

S. c. columbianus (12)

S. richardsonii (7)

Spermophilus spp. (3)

Taxidea t. taxus (17)

Ursus $\operatorname{arctos}(1,5)$

Ursus spp. $(7,12)$

Vulpes vulpes (17)

Chipmunks $(2,5,6,7,18)$

Deer $(7,12,13,18)$

Field mice (2)

Groundhogs $(4,18)$

Ground squirrels $(2,7)$

Guinea pigs $(8,12)$

Lab rabbits $(12,17)$

Mice $(5,7,18)$

Packrats $(5,12)$

Pocket mice (5)

Rabbits $(2,5,7,18)$

Squirrels $(2,5,6)$

Tree squirrels (7)

Weasels (7)

White mice (12)

Unspecified (10)

Dist.: Alta, BC, Man, Ont

Records: 1. Hewitt 1915 (BC); 2. Bruce 1920 (BC); 3. McLeod 1933 (BC, Man); 4. Buckell 1934 (BC); 5. Hearle 1938 (BC); 6. Holland 1938 (BC), 7. 1939 (Alta); 8. Gregson 1941c exp. (BC); 9. Cowan 1944 (Alta); 10. Brown and Kohls 1950 (Alta); 11. Banfield 1956 (Alta); 12. Gregson 1958 exp. (BC); 13. Stelfox 1962 (Alta); 14. Wilkinson 1965 (BC); 15. Kerr and Holmes 1966 (Alta); 16. Shemanchuk and Kiceniuk 1970 (Alta); 17. Wilkinson 1970 nat./exp. (Alta, BC); 18. Ko 1972b (Ont); 19. Samuel et al. 1978 (Alta) 
Remarks: Wilkinson (1970) presumed the subspecies of porcupine from Alberta was $E$. $d$. epixanthium.

Dermacentor variabilis (Say, 1821) Banks, 1907

Hosts: Blarina brevicauda pallida (1)

Clethrionomys gapperi $(3,4,5)$

C. g. pallexens (1)

Condylura cristata nigra (1)

Erethizon dorsatum $(1,4)$

Lepus americanus $(3,4)$

Microtus pennsylvanicus $(3,4,5)$

M. p. acadicus (1)

Napaeozapus insignis $(3,4)$

Ondatra zibethicus (4)

O. z. zibethicus (1)

Peromyscus leucopus $(1,3,4)$

$P$. maniculatus $(4,5)$

P. m. abietorum (1)

Peromyscus spp. (1)

Procyon lotor $(1,4)$

Spermophilus franklinii (5)

$S$. tridecemlineatus (5)

Synaptomys cooperi $(1,4)$

Tamiasciurus hudsonicus (3)

Tamias striatus $(3,4,5)$

Ursus americanus (4)

U. a. americanus (1)

Zapus hudsonius $(3,4,5)$

Z. h. acadicus (1)

Unspecified (2)

Dist.: Man, NS

Records: 1. Dodds et al. 1969 (NS); 2. Martel et al. 1969 (NS); 3. Garvie et al. 1978 (NS); 4.

Wright 1979 (NS); 5. Burachynsky and Galloway 1985 (Man)

Haemaphysalis cinnabarina Koch, 1844

Hosts: Rabbits $(1,2)$

Squirrels (2)

Dist.: BC

Records: 1. Hewitt 1915; 2. Hearle 1938

Remarks: Brown and Kohls (1950) refer

Hearle's (1938) specimens to Haemaphysalis chordeilus (Packard).

Haemaphysalis leporis-palustris (Packard, 1869)

Neumann, 1897

Hosts: Clethrionomys gapperi pallescens $(10)$

Lepus americanus $(1,2,5,7,8,9,11,12$,

13)

L. a. struthopus (10)

L. a. virginianus (4)

L. capensis hybridus (6)

L. townsendii (5)

Microtus pennsylvanicus (12)

M. p. acadicus (10)

Odocoileus hemionus (7)

Spermophilus richardsonii (5)

Tamiasciurus hudsonicus (12)

T. h. gymnicus (10)

Tamias striatus (13)

Chipmunks (3)
Groundhogs (3)

Hare (3)

Rabbits $(2,3,4)$

Squirrels (2)

Dist.: Alta, BC, Man, NB, NS, Ont, PEI, Que,

Sask

Records: 1. Hewitt 1915 (Man); 2. Cameron and Fulton 1927 (Sask); 3. Hearle 1938 (BC); 4. Bequaert 1945 (NB,NS,Ont, Que); 5. Brown and Kohls 1950 (Alta); 6. Judd 1953 (Ont); 7. Scholten et al. 1962 (Ont); 8. Wilson 1967 (NB); 9. Dodds et al. 1969 (NS); 10. Martell et al. 1969 (NS); 11. Ko 1972b nat./exp. (Ont); 12. Wright 1979 (NS); 13. Jones and Thomas 1980 (PEI)

Ixodes angustus Neumann, 1899

Hosts: Blarina brevicauda $(5,9,10)$

B. b. pallida (7)

Clethrionomys gapperi $(9,10)$

C. g. pallescens (7)

Clethrionomys spp. (3)

Condylura cristata (10)

Microsorex hoyi (10)

Microtus chrotorrhinus (10)

M. pennsylvanicus (9)

M. p. acadicus (7)

Napaeozapus insignis (9)

Ochotona spp. (1)

Peromyscus leucopus (9)

P. maniculatus (10)

Peromyscus spp. (4)

Sorex cinereus (10)

S. c. acadicus (7)

S. fumeus $(9,10)$

S. gaspensis $(10)$

S. palustris (10)

Sorex spp. (1)

Synaptomys borealis (10)

S. cooperi (10)

Tamiasciurus douglasii mollipilosus (2)

T. hudsonicus (8)

Tamias striatus (6)

Zapus hudsonius (10)

Chipmunk (1)

Mice (1)

Rabbit (1)

Squirrel (1)

Vole (1)

Dist.: Alta, BC, NB, NS, Ont

Records: 1. Hearle 1938 (BC); 2. Gregson

$1941 \mathrm{~b}$ (BC); 3. Brown and Kohls 1950 (Alta); 4. Judd 1950 (Ont), 5. 1953 (Ont); 6. Wilson 1967

(Ont); 7. Martell et al. 1969 (NS); 8. Mahrt and Chai 1972 (Alta); 9. Wright 1979 (NS); 10.

Whitaker and French 1982 (NB)

Ixodes californicus Banks, 1904

Hosts: Odocoileus hemionus columbianus (2)

Peromyscus spp. (1)

Squirrel (1)

Dist.: BC

Records: 1. Gregson 1942 exp.; 2. Cowan 1946 
Remarks: Gregson's (1942) specimens were misidentified and should be I. pacificus (Arthur and Snow 1968).

Ixodes cookei Packard, 1869

Syn.: Ixodes hexagonus var. cookei Nuttall and Warburton, 1911

Hosts: Castor canadensis (6)

Erethizon dorsatum (12)

E. d. dorsatum (8)

Marmota monax $(6,7,9,10,11)$

M. m. rufescens $(4,5)$

Mephitis mephitis $(5,6,7)$

Mustela erminea (12)

$M$. vison $(4,13)$

M. v. vison (8)

Procyon lotor (12)

Deer (3)

Groundhog $(1,2)$

Packrat (2)

Squirrel (3)

Mouse (3)

Dist.: BC, NS, Ont, Que

Records: 1. Jarvis 1910 (Ont); 2. Hearle 1938

(BC); 3. Gregson 1942 (BC); 4. Bequaert 1945

(NS, Ont, Que); 5. Judd 1950 (Ont), 6. 1954a

(Ont); 7. Scholten et al. 1962 (Ont); 8. Martell

et al. 1969 (NS); 9. Ko"1972a (Ont), 10. 1972b

(Ont), 11. 1973 (Ont); 12. Wright 1979 (NS); 13.

Jones and Thomas 1980 (Que)

Ixodes hearlei Gregson, 1941

Host: Tamiasciurus hudsonicus streatori

Dist.: BC

Record: Gregson 1941b

Ixodes hexagonus Leach, 1815

Host: Weasel

Dist.: BC

Record: Hearle 1938

Ixodes kingi Bishopp, 1911

Hosts: Mephitis mephitis (2)

Mustela erminea (2)

$M$. frenata nevadensis (2)

Spermophilus richardsonii (1)

Taxidea taxus (1)

Dist.: Alta, BC, Sask

Records: 1. Brown and Kohls 1950 (Alta); 2.

Wilkinson 1970 (BC, Sask)

Ixodes marmotae Cooley and Kohls, 1938

Host: Mephitis mephitis

Dist.: BC

Record: Wilkinson 1970

Ixodes marxi Banks, 1908

Hosts: Glaucomys sabrinus (6)

G. s. gouldi (5)

Sorex cinereus $(7)$

Tamiasciurus hudsonicus $(4,6,8)$

T. h. loquax (3)

Chipmunk (2)

Squirrel $(1,2)$
Weasel (2)

Dist.: BC, NS, Ont, PEI, Que

Records: 1. Jarvis 1910 (Ont); 2. Hearle 1938

(BC); 3. Bequaert 1945 (Ont); 4. Scholten et al.

1962 (Ont); 5. Martell et al. 1969 (NS); 6.

Wright 1979 (NS); 7. Jones and Thomas 1980

(PEI); 8. Gyorkos and Hilton 1982 (Que)

Ixodes muris Bishopp and Smith, 1937

Hosts: Blarina brevicauda (4)

Clethrionomys gapperi (4)

Microtus pennsylvanicus $(3,4)$

M. p. acadicus (2)

$M . p$. enixus (1)

Napaeozapus insignis (3)

N. i. insignis (1)

Peromyscus leucopus caudatus (2)

$P$. maniculatus (4)

Sorex arcticus (4)

S. cinereus $(3,4)$

S. c. acadicus (2)

Synaptomys cooperi (3)

S. c. cooperi (2)

Tamiasciurus hudsonicus (3)

Tamias striatus (3)

Zapus hudsonius $(3,4,5)$

Z. h. acadicus (2)

Dist.: NB, Nfld, NS, PEI

Records: 1. Bequaert 1945 (Nfld, NS); 2.

Martell et al. 1969 (NS); 3. Wright 1979 (NS);

4. Jones and Thomas 1980 (NB, NS, PEI), 5.

1982 (NB, NS, PEI)

Ixodes ochotonae Gregson, 1941

Hosts: Neotoma cinerea

Ochotona princeps

O. p. brooksi

O. p. brunnescens

Dist.: BC

Peromyscus spp.

Record: Gregson 1941b

Ixodes pacificus Cooley and Kohls, 1943

Hosts: Eutamias spp. (2)

Glaucomys sabrinus oregonensis (1)

Mustela vison (2)

Odocoileus hemionus (2)

$O$. h. columbianus (2)

Peromyscus spp. (2)

Scapanus latimanus (2)

Tamiasciurus spp. (2)

Coast deer (2)

Mouse (2)

Dist.: $\mathrm{BC}$

Records: 1. Spencer 1956; 2. Arthur and Snow 1968

Ixodes ricinus (Linnaeus, 1758)

Host: Coast deer

Dist.: BC

Record: Hearle 1938

Remarks: According to Arthur and Snow (1968), Hearle's (1938) specimens of I. ricinus are misidentified, and are correctly I. pacificus. 
Ixodes rugosus Cooley and Kohls, 1945

Host: Canis latrans

Dist.: BC

Record: Wilkinson 1970

Ixodes scapularis Say, 1821

Host: Odocoileus virginianus

Dist.: Ont

Record: Watson and Anderson 1976

Ixodes sculptus Neumann, 1904

Hosts: Canis latrans (3)

Mustela frenata (1)

Spermophilus columbianus (1)

S. franklinii (4)

S. richardsonii $(1,4)$

Ground squirrel (2)

Dist.: Alta, BC, Sask

Records: 1. Brown and Kohls 1950 (Alta); 2.

MacNay 1952 (Sask); 3. Wilkinson 1970 (BC);

4. Hilton and Mahrt 1971 (Alta)

Ixodes soricis Gregson, 1942

Hosts: Sorex vagrans

Dist.: BC

S. v. vagrans

Sorex spp.

Record: Gregson and Kohls 1952

Ixodes spinipalpus Hadwen and Nuttall, 1916

Syn.: Ixodes dentatus spinipalpis Hadwen and Nuttall, 1916

Hosts: Lepus americanus (1)

Neotoma c. cinerea (4)

Ochotona sp. (2)

Sorex cinereus (3)

Tamiasciurus douglasii (1)

Rabbit (2)

Squirrel (2)

Dist.: Alta, BC

Records: 1. Nuttall 1916 (BC); 2. Hearle 1938

(BC); 3. Brown and Kohls 1950 (Alta); 4.

Shemanchuk and Kiceniuk 1970 (Alta)

Remarks: Brown and Kohls (1950) believed the shrew was $S$. cinereus, but they were not certain.

Ixodes texanus Banks, 1909

Hosts: Marmota monax (6)

Mephitis mephitis (5)

Mustela vison (4)

Procyon lotor $(1,5)$

Vulpes vulpes (5)

Mustelidae gen. sp. (3)

Mink (2)

Squirrel (1)

Weasel $(1,2)$

Dist.: BC, Ont

Records: 1. Hearle 1938 (BC); 2. Gregson

$1941 \mathrm{~b}$ (BC), 3. 1941c exp. (BC); 4. Bequaert

1945 (Ont); 5. Webster 1966 (Ont); 6. Ko 1972 b

(Ont)
Ixodes spp.

Hosts: Clethrionomys gapperi (1)

Erethizon dorsatum (2)

Peromyscus maniculatus (1)

Dist.: BC, Ont

Records: 1. Scholten et al. 1962 (Ont); 2.

Wilkinson 1970 (BC)

Unidentified Ixodidae

Ixodidae gen. sp.

Hosts: Rodents

Dist.: Alta, BC

Record: Gibbons 1939

Unidentified Chelicerata

Acari gen. spp.

Host: Glaucomys sabrinus oregonensis

Dist.: BC

Record: Spencer 1956

\section{PHYLUM UNIRAMIA}

\section{CLASS INSECTA}

\section{ORDER COLEOPTERA}

\section{Family LEPTINIDAE}

Leptinillus aplodontiae (Ferris, 1918)

Syn.: Leptinus aplodontiae Ferris, 1918

Hosts: Aplodontia pacifica (2)

Dist.: BC

Aplodontia spp. (1)

Records: 1. Hopping 1949; 2. Spencer 1957

Leptinillus validus (Horn, 1872)

Syn.: Leptinus validus Horn, 1872

Hosts: Castor canadensis $(1,3,4,5)$

Beaver (2)

Dist.: BC, Hudson's Bay Area, Ont, Que

Records: 1. Horn 1872 (Hudson's Bay Area); 2.

Hopping 1949 (BC); 3. Judd 1954a (Ont); 4.

Parks and Barnes 1955 (BC, Ont, Que); 5.

Spencer 1957 (BC)

Remarks: Horn (1872) did not specify the host, we assumed it was Castor canadensis.

Leptinus americanus LeConte, 1866

Hosts: Blarina brevicauda (2)

Microtus oregoni serpens (3)

Sorex spp. $(1,3)$

Dist.: BC, Ont

Records: 1. Hopping 1949 (BC); 2. Jameson 1950 (Ont); 3. Spencer 1957 (BC)

Remarks: Spencer (1957) notes that Hopping (1949) recorded his material from Sorex sp. as Leptinus testaceus Muller, 1817. It is in fact $L$. americanus. Gregson (1940), cited in Hopping (1949), also reported L. testaceus from Sorex sp. from BC. 
Platypsyllus castoris Ritsema, 1869

Hosts: Castor canadensis $(3,4)$

Beaver $(1,2)$

Dist.: BC, Ont

Records: 1. Anon 1930 (Ont); 2. Hopping 1949

(BC); 3. Spencer 1957 (BC); 4. Scholten et al. 1962 (Ont)

\section{ORDER DIPTERA}

\section{Family CALLIPHORIDAE}

Phormia regina (Meigen, 1826)

Host: Odocoileus hemionus columbianus

Dist.: BC

Record: Cowan 1946

\section{Family CUTEREBRIDAE}

Cuterebra americana var. polita Coquillett, 1898

Host: unspecified

Dist.: BC

Record: Cameron 1926

Cuterebra angustifrons Dalmat, 1942

Hosts: Peromyscus leucopus (2)

Dist.: Ont

$$
P \text {. l. noveboracensis (1) }
$$

Records: 1. Sillman 1957; 2. Sillman and Smith 1959

\section{Cuterebra emasculator Fitch, 1856}

Hosts: Tamias striatus $(2,3,4,5)$

Dist.: Ont

$$
\text { T. s. lysteri (1) }
$$

Records: 1. Cameron 1926 (unspecified); 2.

Bennett 1955; 3. Scholten et al. 1962; 4. Bennett 1972, 5. 1973

Cuterebra fasciata Swenk, 1905

Host: unspecified

Dist.: BC, NB

Record: Cameron 1926

Remarks: The larvae were found in subcutaneous tissues.

Cuterebra fontinella Clark, 1827

Host: Mus musculus

Dist.: Ont

Record: Judd 1954b

Remarks: The larva emerged from the skin leaving a hole that penetrated the underlying muscle of the abdominal wall and led into the body cavity (Judd 1954b).

\section{Cuterebra grisea Coquillett, 1904}

Hosts: Peromyscus leucopus noveboracensis (3)

P. maniculatus (4)

P. m. austerus (5)

Spermophilus richardsonii (1)

$S$. tridecemlineatus (1)

Field mouse

House mouse (1)

Mice (2)
Dist.: Alta, BC, Man, NWT, Ont, Sask

Records: 1. Cameron 1926 (Alta, BC, Man, NWT, Sask); 2. Strickland 1938 (Alta); 3.

Sillman 1957 (Ont); 4. Hunter et al. 1972 (BC);

5. Hunter and Webster 1974 (BC)

Remarks: The larvae were found in subcutaneous tissues. Cameron's (1926) host and distribution records are not well defined.

Cuterebra similis Johnson, 1903

Host: unspecified

Dist.: BC

Record: Cameron 1926

Cuterebra tenebrosa Coquillet, 1898

Host: unspecified

Dist.: BC

Record: Cameron 1926

Cuterebra spp.

Hosts: Microtus oregoni (6)

M. townsendii (7)

Mus musculus (3)

Peromyscus leucopus noveboracensis

$(2,3,4)$

$P$. maniculatus (5)

P. m. gracilis (3)

$P$. m. maniculatus (3)

Rattus norvegicus (1)

Rattus spp. (1)

Sylvilagus floridanus mearnsii (2)

Tamias striatus (3)

Gopher (1)

House mouse (1)

Rabbit (3)

Vole (1)

Dist.: BC, Man, Ont, Sask

Records: 1. Cameron 1926 (Man, Sask, unspecified); 2. Jameson 1943 (Ont); 3. Sillman 1956 (Ont); 4. Sealander 1961 (Ont); 5.

Scholten et al. 1962 (Ont); 6. Hunter et al. 1972 (BC); 7. Boonstra 1977 (BC)

\section{Family HIPPOBOSCIDAE}

Lipoptena depressa (Say, 1823) Sacken, 1878

Hosts: Odocoileus hemionus $(2,9)$

$O$. h. columbianus $(3,4,5,6,7,8)$

$O$. virginianus $(9)$

Dist.: BC

Odocoileus spp. (1)

Records: 1. Ferris and Cole 1922; 2. Bequaert 1935, 3. 1937; 4. Spencer 1938a, 5. 1938b; 6.

Bequaert 1942; 7. Cowan 1943, 8. 1946; 9.

Bequaert 1957

Remarks: Bequaert (1937) recorded the host as the Columbian white-tailed deer by error (Bequaert 1942).

Melophagus ovinus montanus Ferris and Cole, 1922

Hosts: Canis latrans (2) Ovis canadensis (1)

Dist.: Alaska-Yukon boundary, BC 
Records: 1. Ferris and Cole 1922 (AlaskaYukon boundary); 2. Bequaert 1942 (BC)

Remarks: Bequaert (1942) did not recognize $M$. o. montanus as a distinct subspecies.

Neolipoptena ferrisi (Bequaert, 1935) Bequaert, 1942

Syn.: Lipoptena ferrisi Bequaert, 1935

Hosts: Odocoileus hemionus $(1,7)$

$$
\begin{aligned}
& O . h \text {. columbianus }(3,4,6) \\
& O . \text { h. hemionus }(3,4,5) \\
& \text { O. virginianus }(1)
\end{aligned}
$$

Dist.: BC

Records: 1. Bequaert 1935, 2. 1937; 3. Spencer 1938a, 4. 1938b; 5. Bequaert 1942; 6. Cowan

1946; 7. Bequaert 1957

\section{Family NYCTERIBIIDAE}

Basilia forcipata Ferris, 1924

Hosts: Myotis lucifugus (2)

$$
\text { Bat (1) }
$$

Dist.: BC

Record: Spencer 1938b, 2. 1939a

Basilia spp.

Host: Eumops perotis californica

Dist.: BC

Record: Spencer 1939a

\section{Family OESTRIDAE}

Cephenemyia apicata Bennett and Sabrosky, 1962

Host: Odocoileus hemionus

Dist.: Alta, BC

Record: Bennett and Sabrosky 1962

Cephenemyia jellisoni Townsend, 1941

Hosts: Alces alces (4)

Cervus elaphus (5)

Odocoileus hemionus $(2,4)$

$O$. h. columbianus $(1,3)$

O. h. hemionus (1)

Dist.: Alta, BC, Ont

Records: 1. Cowan 1943 (BC), 2. 1944 (Alta), 3.

1946 (BC); 4. Bennett and Sabrosky 1962 (BC,

Ont); 5. Flook and Stenton 1969 (Alta)

Cephenemyia phobifer (Clark, 1815) Aldrich, 1915

Hosts: Alces alces (3)

Odocoileus virginianus (2)

Rangifer tarandus caribou (1)

Dist.: Ont, Sask

Records: 1. Erickson and Highby 1942 (Sask);

2. Bennett 1962 (Ont); 3. Bennett and Sabrosky 1962 (Ont)

Remarks: The larvae were found in the nasal passages and retropharyngeal pouches.

Cephenemyia trompe (Modeer, 1786)

Syn.: Cephenemyia nasalis Seguy, 1927

Hosts: Odocoileus virginianus (5)

Rangifer tarandus $(3,4,5)$

R. t. caribou (2)
Dist.: Alta, BC, Man, Nfld, NWT, Ont, Sask

Records: 1. Buckell 1934 (BC); 2. Cowan 1944

(Alta, BC); 3. Banfield 1954 (Man, NWT); 4.

Gibbs 1960 (unspecified); 5. Bennett and

Sabrosky 1962 (Nfld, NWT, Ont, Sask)

Remarks: The larvae were found in the throat and gullet (Buckell 1934).

Cephenemyia spp.

Hosts: Alces alces $(1,2)$ Odocoileus virginianus (3)

Dist.: BC, Ont

Records: 1. Peterson 1955 (Ont); 2. Ritcey and Edwards 1958 (BC); 2. Scholten et al. 1962

(Ont)

Remarks: Ritcey and Edwards (1958) felt their specimens were probably $C$. jellisoni.

Oedemagena tarandi Linnaeus, 1758

Hosts: Rangifer tarandus $(2,3,4)$

$R$. t. groenlandicus (5)

R. t. caribou (1)

Dist.: Alta, BC, Man, NWT, YT

Records: 1. Cowan 1944 (Alta, BC); 2. Banfield 1954 (Man, NWT); 3. Choquette et al. 1957

(NWT); 4. Gibbs 1960 (unspecified); 5. Kelsall

1972 (Alta, BC, NWT, YT)

\section{Family SARCOPHAGIDAE}

Wohlfahrtia vigil (Walder, 1849)

Syn.: Wohlfahrtia meigenii Schiner, 1862

Hosts: Microtus townsendii (3)

Lab rat $(1,2)$

Dist.: Alta, BC

Records: 1. Morrison 1937 (Alta); 2. Strickland 1938 (Alta); 3. Boonstra 1977 (BC)

Remarks: The larvae were recovered from subcutaneous tissues (Morrison 1937).

\section{Family STREBLIDAE}

Trichobius spp.

Host: Bat

Dist.: BC

Record: Spencer 1938b

\section{ORDER HEMIPTERA}

\section{Family CIMICIDAE}

Cimex pilosellus (Horvath)

Hosts: Eptesicus fuscus (3)

E.f. fuscus (1)

E. f. pallidus (1)

Lasionycteris noctivagans $(1,3,4)$

Myotis lucifugus (3)

Myotis l. lucifugus (2)

Dist.: BC, Ont

Records: 1. Spencer 1934 (BC); 2. Dymond 1938 (Ont); 3. Spencer 1939a (BC); 4. Judd 1950 (Ont) 


\section{ORDER MALLOPHAGA}

Family TRICHODECTIDAE

Damalinia concavifrons Hopkins, 1960

Hosts: Cervus elaphus (2)

C. e. nelsoni (1)

Dist.: Alta, BC

Records: 1. Hopkins 1960 (Alta); 2. Flook and Stenton 1969 (Alta, BC)

Damalinia longicornis (Nitzsch, 1818)

Hosts: Cervus elaphus (2)

$$
\text { C. e. nelsoni (1) }
$$

Dist.: Alta

Record: 1. Hopkins 1960; 2. Flook and Stenton 1969

Damalinia oreamnidis Hopkins, 1960

Host: Oreamnos americanus missoulae

Dist.: Alta

Record: Hopkins 1960

Damalinia ovis (Schrank, 1781)

Host: Ovis canadensis californianus

Dist.: BC

Record: Hopkins 1960

Damalinia sedecimdecembrii (Eichler, 1946)

Host: Bison bison

Dist.: NWT

Record: Hopkins 1960

Eutrichophilus setosus (Giebel, 1874)

Hosts: Erethizon dorsatum $(1,2,4)$

E. d. nigrescens (3)

Dist.: Alta, BC, NS, Ont

Records: 1. Thompson 1934 (NS); 2. Judd

1954a (Ont); 3. Hopkins 1960 (Alta, BC); 4.

Wright 1979 (NS)

Felicola spenceri Hopkins, 1960

Host: Lynx canadensis canadensis

Dist.: BC

Record: Hopkins 1960

Geomydoecus spp.

Host: Thomomys talpoides

Dist.: Sask

Record: Hopkins 1960

Remarks: This species may be $G$. thomomyus (Hopkins, 1960).

Neotrichodectes mephitidis (Packard, 1872)

Host: Mephitis mephitis

Dist.: Ont

Records: Judd 1953; Scholten et al. 1962

Stachiella kingi (McGregor, 1917)

Syn.: Trichodectes kingi McGregor, 1917

Hosts: Mustela erminea (2)

$M$. nivalis rixosa (1)

Dist.: BC, Ont

Record: 1. Hopkins 1960 (BC); 2. Scholten et al. 1962 (Ont)
Trichodectes canis (de Geer, 1778)

Hosts: Canis latrans incolatus (3)

$$
\begin{aligned}
& \text { C. l. lestes (3) } \\
& \text { C. lupus }(1,2) \\
& \text { C. l. columbianus (3) }
\end{aligned}
$$

Dist.: BC, Ont

Records: 1. Thompson 1934 (Ont); 2. Judd

1954a (Ont); 3. Hopkins 1960 (BC)

Remarks: Thompson (1934) had some doubts as to the correctness of the parasite genus, Trichodectes.

Trichodectes ermineae (Hopkins, 1941)

Hosts: Mustela erminea anguinae

Dist.: BC

M. e. richardsonii

$M$. vison energumenos

Record: Hopkins 1960

Trichodectes minutus Paine, 1912

Host: Mustela frenata oribasus

Dist.: BC

Record: Hopkins 1960

Trichodectes octomaculatus Paine, 1912

Host: Procyon lotor vancouverensis

Dist.: BC

Record: Hopkins 1960

Trichodectes osborni (Keler, 1944)

Host: Spilogale putorius gracilis

Dist.: BC

Record: Hopkins 1960

Trichodectes parallelus (Osborn, 1896)

Host: Odocoileus hemionus

Dist.: Alta

Record: Cowan 1944

Trichodectes pinguis euarctidos Hopkins, 1954

Hosts: Ursus americanus $(1,3)$

U. a. cinnamomum (2)

Dist.: BC, Ont

Records: 1. Hopkins 1954 (BC, Ont), 2. 1960

(BC); 3. Addison et al. 1978 (Ont)

Trichodectes tibialis (Piaget, 1880)

Host: Odocoileus h. hemionus

Dist.: BC

Record: Buckell 1934

Tricholipeurus lipeuroides (Megnin, 1884)

Syn.: Damalinia lipeuroides (Megnin, 1884)

Holland 1960

Tricholipeurus virginianus Peters, 1930

Hosts: Canis latrans incolatus (3)

O. hemionus (7)

$O$. h. columbianus $(1,2,3)$

$O . h$. hemionus $(1,3)$

O. virginianus $(4,6,7)$

O. v. borealis (5)

Dist.: Alta, BC, Ont, Que 
Records: 1. Spencer 1938a (BC); 2. Cowan 1946 (BC); 3. Hopkins 1960 (Alta, BC); 4. Scholten et al. 1962 (Ont); 5. Webster and Stewart 1964 (Que); 6. Watson and Anderson 1975 (Ont); 7. Samuel et al. 1980 (Alta)

Tricholipeurus parallelus (Osborn, 1896)

Hosts: $O$. hemionus $(2,7)$

$$
\begin{aligned}
& \text { O. h. columbianus }(1,3,4) \\
& \text { O. h. hemionus }(1,4) \\
& \text { O. virginianus }(5,6,7) \\
& \text { Odocoileus spp. }(5)
\end{aligned}
$$

Dist.: Alta, BC, NS, Ont

Records: 1. Spencer 1938a (BC); 2. Cowan 1944 (Alta, BC), 3. 1946 (BC); 4. Hopkins 1960 (BC); 5. Walker and Beckland 1970 (NS, Ont); 6. Watson and Anderson 1975 (Ont); 7. Samuel et al. 1980 (Alta)

Tricholipeurus spp.

Host: Odocoileus hemionus columbianus

Dist.: BC

Record: Hopkins 1960

Mallophaga gen. spp.

$$
\text { Unidentified Mallophaga }
$$

Hosts: Cervus elaphus

$$
\text { Oreamnos americanus }
$$

Dist.: Alta, BC

Record: Cowan 1944

\section{ORDER SIPHONAPTERA}

\section{Family CERATOPHYLLIDAE}

Amalaraeus athabascae (Holland, 1952)

Syn.: Malaraeus penicilliger athabascae

Holland, 1952

Hosts: Clethrionomys gapperi $(1,4)$

C. g. athabascae $(1,4)$

C. g. proteus $(1,3,4)$

C. g. ungava $(1,2,3,4)$

Clethrionomys spp. (4)

Microtus pennsylvanicus $(1,4)$

M. p. drummondii (4)

Microtus spp. $(1,4)$

Mustela erminea (4)

Peromyscus maniculatus (4)

Synaptomys borealis $(1,4)$

Dist.: Alta, BC, Man, Nfld, NWT, Que, Sask

Records: 1. Holland 1952b (Alta, Man, Nfld,

NWT, Que); 2. Gabbutt 1961 (Nfld); 3. Harper

1961 (Nfld, Que); 4. Holland 1984 (BC, Man,

Nfld, NWT, Que, Sask)

Amalaraeus dissimilis (Jordan, 1929)

Syn.: Ceratophyllus penicilliger Jordan, 1929

Malaraeus penicilliger dissimilis Jordan, 1938

Hosts: Alopex lagopus (7)

Clethrionomys gapperi $(3,7)$

C. g. athabascae $(3,5,7)$

C. g. saturatus (3)

C. rutilus $(6,7)$
C. r. dawsoni $(4,7)$

Clethrionomys spp. (7)

Eutamias minimus (7)

Lemmus sibiricus (7)

Lepus americanus dalli (7)

Microtus longicaudus (7)

M. l. vellerosus (7)

M. miurus (7)

M. oeconomus (7)

M. operarius (7)

M. pennsylvanicus (7)

M. p. drummondii (7)

M. xanthognathus (7)

Microtus spp. (7)

Mustela erminea (7)

M. e. $\operatorname{arctica}(7)$

Neotoma cinerea occidentalis (7)

Ochotona collaris (7)

Ondatra spp. (7)

Peromyscus maniculatus (7)

P. m. borealis (1)

Peromyscus spp. (7)

Phenacomys intermedius $(3,7)$

Phenacomys spp. (7)

Spermophilus lateralis (7)

Synaptomys borealis (7)

Vulpes vulpes (2)

Vulpes spp. (2)

Mice $(3,7)$

Dist.: Alta, BC, NWT, YT

Records: 1. Jordan 1932 (BC); 2. Holland 1944

(NWT), 3. 1949 (Alta, BC, NWT), 4. 1952b

(BC, YT); 5. Harper 1956 (NWT); 6. Mitchell

and Behin 1965 (NWT); 7. Holland 1984 (Alta, BC, NWT, YT)

Amphalius runatus necopinus (Jordan, 1925) Jordan, 1933

Syn.: Amphalius necopinus (Jordan, 1925)

Hosts: Ochotona princeps $(1,2,3)$ Ochotona spp. (4)

Dist.: Alta, BC

Records: 1. Holland 1941a (Alta, BC); 2.

Brown 1944 (Alta); 3. Holland 1949 (Alta), 4. 1984 (Alta)

Ceratophyllus adustus Jordan, 1932

Host: Erethizon dorsatum

Dist.: BC

Records: Jordan 1932

Ceratophyllus diffinis Jordan, 1925

Host: Eutamias spp.

Dist.: BC

Record: Holland 1984

Ceratophyllus gallinae (Schrank, 1803)

Hosts: Clethrionomys gapperi (1) Glaucomys volans (2) Red squirrel (2)

Dist.: Man

Records: 1. Holland 1949, 2. 1984 
Ceratophyllus garei Rothschild, 1902

Hosts: Canis latrans

Clethrionomys rutilus dawsoni
Clethrionomys spp.
Dicrostonyx hudsonius
Mustela nivalis rixosa
Peromyscus spp.
Sorex vagrans obscurus
Tamiasciurus hudsonicus preblei
Lemming

Dist.: Alta, BC, Man, NWT, Que, YT

Record: Holland 1984

Ceratophyllus lunatus tundrensis Holland, 1944

Hosts: Clethrionomys rutilus dawsoni (2)

Dicrostonyx groenlandicus richardsoni

(2)

D. hudsonius (2)

Dicrostonyx spp. (3)

Martes americana (1)

Microtus spp. (3)

Mustela erminea $(2,3)$

M. e. $\operatorname{arctica}(1)$

M. e. cicognanii (3)

Mustela spp. $(2,3)$

Spermophilus parryii $(2,3)$

S. p. parryii (2)

Tamiasciurus hudsonicus (3)

Red-backed mouse (3)

Weasel (3)

Dist.: Man, Nfld, NWT, Que, YT

Records: 1. Holland 1944 (NWT), 2. 1951

(NWT, Que), 3. 1984 (Man, Nfld, NWT, Que, YT)

Ceratophyllus niger Fox, 1908

Host: Tamiasciurus hudsonicus

Dist.: BC

Record: 1. Holland 1949

Ceratophyllus petrochelidoni Wagner, 1936

Host: Tamiasciurus hudsonicus picatus

Dist.: BC

Record: Holland 1984

Dactylopsylla comis Jordan, 1929

Host: Thomomys talpoides

Dist.: BC

Record: Jordan 1929

Dactylopsylla rara Fox, 1940

Hosts: Mustela erminea

Thomomys talpoides

Dist.: Alta, Sask

Record: Holland 1984

Dasypsyllus gallinulae perpinnatus (Baker, 1904)

Hosts: Microtus oregoni serpens (2)

Peromyscus spp. (2)

Tamiasciurus douglasii mollipilosus (1)

Dist.: BC

Records: 1. Wagner 1936; 2. Holland 1984
Foxella ignota albertensis (Jordan and Rothschild, 1915)

Syn.: Ceratophyllus ignotus albertensis Jordan and Rothschild, 1915

Dactylopsylla ignota albertensis (Jordan and Rothschild, 1915)

Hosts: Geomys spp. $(1,3)$

Lynx canadensis $(1,3)$

Microtus pennsylvanicus drummondii

(5)

Mustela erminea (6)

M. e. $\operatorname{arctica}(6)$

M. frenata $(6)$

Mustela spp. $(1,3)$

Peromyscus maniculatus bairdii (5)

Thomomys talpoides $(4,6)$

T. t. fuscus (2)

Thomomys spp. (6)

Pocket gopher (6)

Weasel (4)

Dist.: Alta, Man, Sask

Records: 1. Jordan and Rothschild 1915 (Alta);

2. Holland 1941a (Alta); 3. Brown 1944 (Alta);

4. Holland 1949 (Alta); 5. Buckner and Blasko

1969 (Man); 6. Holland 1984 (Alta, Sask)

Foxella ignota ignota (Baker, 1895)

Hosts: Pocket gopher

Weasel

Dist.: Man

Record: Holland 1984

Foxella ignota recula (Jordan and Rothschild, 1915)

Syn.: Ceratophyllus ignotus recula Jordan and Rothschild, 1915

Dactylopsylla ignota recula (Jordan and

Rothschild, 1915)

Hosts: Mustela erminea (3)

$M$. frenata $(1,2,3)$

M. f. arizonensis $(2,4)$

Mustela spp. $(1,4)$

Spermophilus c. columbianus (3)

Thomomys talpoides $(1,3,4)$

T. $t$. incensus $(2,4)$

Thomomys spp. (4)

Gopher (4)

Pocket gopher (4)

Dist.: BC

Records: 1. Jordan and Rothschild 1915; 2.

Wagner 1936; 3. Holland 1949, 4. 1984

Malaraeus bitterrootensis (Dunn and Parker, 1923)

Hosts: Mus spp. $(2,3)$

Mustela erminea invicta (3)

Neotoma cinerea (1)

Neotoma spp. (3)

Dist.: Alta, BC

Records: 1. Wagner 1936a (BC); 2. Brown 1944

(Alta); 3. Holland 1984 (Alta) 
Malaraeus euphorbi (Rothschild, 1905)

Syn.: Ceratophyllus euphorbi Rothschild, 1905

Hosts: Clethrionomys spp. (4)

Mustela frenata longicauda (3)

Peromyscus maniculatus $(1,2,3,4)$

P. m. artemisiae (3)

P. m. nebrascensis (3)

Peromyscus spp. (4)

Tamiasciurus hudsonicus baileyi (4)

Deer mouse (4)

Dist.: Alta, BC, NWT, Sask

Records: 1. Rothschild 1905 (BC); 2. Wagner

1936a (BC); 3. Holland 1949 (Alta, BC, Sask),

4. 1984 (Alta, BC, NWT, Sask)

Malaraeus telchinus (Rothschild, 1905)

Syn.: Ceratophyllus telchinum Rothschild, 1905

Hosts: Clethrionomys gapperi $(2,3)$

C. g. saturatus (1)

Clethrionomys spp. (3)

Microtus longicaudus $(2,3)$

$M$. montanus canescens (3)

Microtus spp. (3)

Mus musculus (3)

Mus spp. (3)

Mustela spp. (3)

Peromyscus maniculatus $(2,3)$

P. m. artemisiae $(2,3)$

$P$. m. austerus $(2,3)$

P. m. borealis (2)

P. m. interdictus (2)

P. m. oreas (2)

P. sitkensis (3)

Peromyscus spp. $(2,3)$

Phenacomys intermedius (3)

Sorex spp. $(1,2)$

Spermophilus columbianus (2)

Zapus spp. (3)

Mice $(2,3)$

Red-backed vole (3)

Shrew (3)

Dist.: Alta, BC

Records: 1. Rothschild 1905 (BC); 2. Holland

1949 (BC), 3. 1984 (Alta, BC)

Megabothris abantis (Rothschild, 1905)

Syn.: Ceratophyllus abantis Rothschild, 1905

Trichopsylla abantis (Rothschild, 1905)

Hosts: Clethrionomys gapperi $(6,7)$

C. g. saturatus (6)

Clethrionomys spp. (7)

Eutamias spp. (6)

Lepus americanus $(6,7)$

Microtus longicaudus $(6,7)$

M. l. macrurus $(6,7)$

M. oregoni serpens $(2,6,7)$

$M$. pennsylvanicus (7)

M. p. drummondii $(1,7)$

M. richardsoni (7)

M. r. richardsoni (6)

M. townsendii (2)
M. t. townsendii (7)

Microtus spp. $(6,7)$

Mustela erminea (6)

M. e. cicognanii (4)

M. e. fallenda (6)

M. frenata $(1,2,5,7)$

M. f. oribasa $(6,7)$

Mustela spp. (7)

Neotoma cinerea $(6,7)$

N. c. drummondii (7)

N. c. occidentalis (6)

Ochotona princeps (6)

O. p. princeps (6)

Ochotona spp. (7)

Peromyscus maniculatus $(3,6,7)$

P. m. alpinus (7)

P. m. artemisiae (6)

$P$. m. austerus (6)

P. m. borealis (6)

P. m. oreas (6)

Peromyscus spp. $(6,7)$

Phenacomys intermedius $(6,7)$

P. i. intermedius (6)

Rattus norvegicus $(4,6,7)$

Sorex spp. (7)

Spermophilus c. columbianus (6)

Synaptomys borealis truei (6)

Synaptomys spp. $(6,7)$

Tamiasciurus hudsonicus (7)

T. h. streatori (2)

Thomomys spp. (7)

Vulpes vulpes (6)

Zapus hudsonius (7)

Z. princeps idahoensis (6)

Z. t. trinotatus (6)

Zapus spp. $(6,7)$

Chipmunk (7)

Mouse (6)

Dist.: Alta, BC, YT

Records: 1. Rothschild 1905 (Alta, BC); 2.

Wagner 1936a (BC), 3. 1936b (BC); 4. Holland

1942 (Alta, BC); 5. Brown 1944 (Alta); 6.

Holland 1949 (Alta, BC), 7. 1984 (Alta, BC, YT)

Megabothris acerbus (Jordan, 1925) Jordan, 1933

Syn.: Ceratophyllus acerbus Jordan, 1925

Trichopsylla acerba (Jordan, 1925)

Hosts: Clethrionomys spp. (5)

Eutamias minimus (5)

Sciurus carolinensis (5)

Tamias striatus $(1,4,5)$

T. s. griseus (3)

T. s. lysteri (2)

Tamias spp. (5)

Chipmunk $(3,5)$

Dist.: Man, Ont

Records: 1. Jordan 1925 (unspecified); 2.

Jameson 1943 (Ont); 3. Holland 1949 (Ont); 4.

Scholten et al. 1962 (Ont); 5. Holland 1984

(Man, Ont) 
Megabothris asio (Baker, 1904)

Syn.: Ceratophyllus asio Baker, 1904

Hosts: Clethrionomys gapperi (3)

C. g. ungava (2)

Microtus pennsylvanicus $(3,5)$

M. p. drummondii (1)

M. p. enixus (2)

Phenacomys intermedius celatus (2)

Zapus hudsonicus $(4,5)$

Dist.: NB, Nfld, NS, NWT, Ont

Records: 1. Harper 1956 (NWT); 2. Gabbutt 1961 (Nfld); 3. Scholten et al. 1962 (Ont); 4.

Jones and Thomas 1982 (NS); 5. Whitaker and French 1982 (NB)

Megabothris asio asio (Baker, 1904) Jordan, 1933

Syn.: Trichopsylla asio asio (Baker, 1904)

Hosts: Blarina brevicauda (4)

Blarina spp. (11)

Clethrionomys gapperi (11)

C. g. proteus $(3,11)$

C. g. ungava (11)

Condylura cristata $(9,10,11)$

Mephitis mephitis (4)

Microtus pennsylvanicus $(7,8,9,11)$

M. p. enixus (6)

M. p. pennsylvanicus (2)

M. p. terraenovae (3)

Microtus spp. (11)

Mus musculus (11)

Mustela erminea (11)

Mustela spp. $(1,5,11)$

Napaeozapus insignis (9)

Peromyscus spp. (11)

Phenacomys intermedius celatus (11)

Phenacomys spp. (11)

Rattus spp. (11)

Sorex spp. (11)

Synaptomys borealis medioxinus (6)

S. cooperi $(9,11)$

Synaptomys spp. (11)

Zapus hudsonius $(9,11)$

Zapus spp. (11)

Lemming (11)

Meadow vole (11)

Dist.: Alta, NB, Nfld, NS, Ont, PEI, Que

Records: 1. Brown 1944 (Alta); 2. Baker 1946

(Que); 3. Holland 1950 (Nfld, Que); 4. Judd

1953 (Ont); 5. Brown 1955 (NB); 6. Harper

1961 (Nfld); 7. Robert 1962 (Que); 8. Brown

1968 (NB); 9. Wright 1979 (NS); 10. Whitaker

and French 1982 (NB); 11. Holland 1984 (NB,

Nfld, NS, Ont, PEI, Que)

Megabothris asio megacolpus (Jordan, 1929)

Hosts: Blarina brevicauda manitobensis (5)

Clethrionomys gapperi $(4,5)$

C. g. athabascae (5)

C. g. loringi (5)

C. rutilus (5)

C. r. dawsoni (5)

Clethrionomys spp. (5)
Dicrostonyx groenlandicus richardsoni

$(3,5)$

D. hudsonicus (3)

Dicrostonyx spp. $(3,5)$

Eutamias minimus borealis (3)

Eutamias spp. (5)

Microtus ochrogaster (5)

$M$. oeconomus (5)

M. pennsylvanicus $(3,5)$

M. p. drummondii $(1,3,5)$

M. p. labradorius (3)

Microtus spp. $(3,5)$

Mustela erminea (5)

M. frenata (5)

Mustela spp. $(2,3,5)$

Peromyscus maniculatus (5)

P. m. bairdii (5)

P. m. borealis $(1,3)$

Peromyscus spp. (5)

Phenacomys spp. (5)

Sorex palustris (5)

Spermophilus $r$. richardsonii (3)

Synaptomys borealis (5)

Synaptomys spp. (5)

Tamias striatus (4)

T. s. griseus (5)

Tamiasciurus hudsonicus (5)

Zapus hudsonius (5)

Zapus spp. (3)

Weasel (5)

Dist.: Alta, BC, Man, NWT, Ont, Que, Sask, YT Records: 1. Jordan 1929 (BC); 2. Brown 1944 (Alta); 3. Holland 1950 (Alta, BC, Man, NWT, Ont, Que, Sask); 4. Buckner 1964 (Man); 5. Holland 1984 (Alta, BC, Man, NWT, Ont, Que, Sask, YT)

Remarks: Holland (1984) re-examined Browns' (1944) specimens of Trichopsylla asio asio and found them to be Megabothris asio megacolpus.

Megabothris atrox (Jordan, 1925)

Syn.: Ceratophyllus atrox Jordan, 1925

Hosts: Martes americana (3)

Mustela erminea (4)

M. vison (5)

Mustela spp. $(1,2,5)$

Spermophilus franklinii (5)

Marten (5)

Mink (5)

Dist.: Alta, Man, Ont

Records: 1. Jordan 1925 (Alta); 2. Brown 1944

(Alta); 3. DeVos 1957 (Ont); 4. Wilson 1967

(Ont); 5. Holland 1984 (Alta, Man, Ont)

Megabothris calcarifer gregsoni Holland, 1950

Hosts: C. rutilus $(2,3)$

C. r. dawsoni $(1,3)$

Clethrionomys spp. (3)

Dicrostonyx groenlandicus (3)

Dicrostonyx spp. (3)

Lemmus sibiricus $(1,3)$ 
Lemmus spp. (3)

Lepus americanus dalli (3)

Microtus longicaudus (3)

M. miurus (3)

M. oeconomus (3)

M. pennsylvanicus (3)

M. p. drummondii $(1,3)$

M. xanthognathus (3)

Microtus spp. $(1,3)$

Mustela erminea (3)

$M$. nivalis rixosa (3)

Peromyscus maniculatus (3)

Synaptomys borealis (3)

Dist.: NWT, YT

Records: 1. Holland 1950 (NWT); 2. Mitchell and Behin 1965 (NWT); 3. Holland 1984

(NWT, YT)

Megabothris clantoni clantoni Hubbard, 1949

Syn.: Megabothris obscurus Holland, 1949

Host: Lagurus curtatus

Dist.: BC

Record: Holland 1984

Megabothris groenlandicus (Wahlgren, 1903)

Syn.: Megabothris immitis (Jordan)

Hosts: Clethrionomys rutilus (2)

Dicrostonyx groenlandicus (1)

D. g. kilangmiutak (1)

D. g. richardsoni $(1,2)$

D. hudsonius (2)

Dicrostonyx spp. $(1,2)$

Lemmus sibiricus (2)

L. s. trimucronatus $(1,2)$

Lemmus spp. $(1,2)$

Microtus miurus (2)

M. pennsylvanicus $(1,2)$

$M$. xanthognathus (2)

Microtus spp. $(1,2)$

Mustela erminea $(1,2)$

Mustela spp. (2)

Peromyscus spp. (2)

Spermophilus parryii $(1,2)$

Synaptomys spp. (2)

Dist.: Man, Nfld, NWT, Que, YT

Records: 1. Holland 1952a (Man, NWT, Que),

2. 1984 (Man, Nfld, NWT, Que, YT)

Megabothris lucifer (Rothschild, 1905)

Syn.: Ceratophyllus lucifer Rothschild, 1905

Hosts: Clethrionomys richardsoni (6)

Eutamias spp. (6)

Microtus montanus canescens $(3,6)$

M. ochrogaster minor (6)

M. pennsylvanicus (6)

M. p. drummondii $(1,4,5)$

Microtus spp. $(2,6)$

Mustela frenata (5)

M.f. longicauda (5)

M. f. nevadensis (6)

Peromyscus maniculatus nebrascensis

(5)

Peromyscus spp. (6)
Spermophilus $r$. richardsonii (5)

Synaptomys spp. (6)

Dist.: Alta, BC, Man, Sask

Records: 1. Rothschild 1905 (Alta); 2. Wagner 1936 (BC); 3. Holland 1941a (BC); 4. Brown

1944 (Alta); 5. Holland 1949 (Alta, BC, Sask),

6. 1984 (Alta, BC, Man, Sask)

Megabothris quirini (Rothschild, 1905)

Syn.: Ceratophyllus quirini Rothschild, 1905

Trichopsylla quirini (Rothschild, 1905)

Hosts: Blarina brevicauda $(18,20)$

B. b. manitobensis (17)

Clethrionomys gapperi $(7,8,13,14,16$, $18,20)$

C. g. athabascae $(7,10,20)$

C. g. gapperi $(7,12)$

C. g. gaspeanus (16)

C. g. loringi $(1,6,14,20)$

C.g.proteus $(12,20)$

C. g. saturatus $(1,7)$

C. g. ungava $(11,12,20)$

C. rutilus (20)

C. r. dawsoni $(9,20)$

Clethrionomys spp. (20)

Dicrostonyx hudsonius $(11,20)$

Dicrostonyx spp. (20)

Eutamias minimis $(14,20)$

E. m. borealis $(7,14)$

Eutamius spp. (20)

Lemmus sibiricus (20)

Mephitis m. mephitis (7)

Microtus chrotorrhinus (18)

$M$. longicaudus (20)

$M$. l. vellerosus $(20)$

$M$. ochrogaster (20)

$M$. oeconomus (20)

M. o. operarius (20)

M. pennsylvanicus $(13,14,18,20)$

M. p. drummondii $(3,5,7,10,14,20)$

M. p. enixus (11)

M. p. insperatus (7)

M. p. labradorius (20)

M. p. pennsylvanicus (7)

Microtus spp. $(7,20)$

Mustela erminea $(7,15,20)$

M. e. richardsonii (20)

M. frenata longicauda (7)

Napaeozapus insignis $(13,18,20)$

$N$. i. frutectanus $(7)$

$N$. i. insignis $(16,20)$

Napaeozapus spp. (20)

Neotoma cinerea $(20)$

Ochotona collaris (20)

Peromyscus leucopus $(18,20)$

P. maniculatus $(7,13,16,18,20)$

P. m. algidus (20)

P. m. artemisiae (7)

P. m. bairdii $(14,20)$

$P$. $m$. borealis $(7,20)$

Peromyscus spp. (20)

Phenacomys intermedius celatus $(11,20)$ 
Phenacomys spp. (20)

Rattus norvegicus (13)

Sorex cinereus (20)

S. c. cinereus $(14,20)$

Spermophilus franklinii (7)

S. parryii (20)

Sylvilagus nuttallii grangeri (7)

Synaptomys borealis $(19,20)$

S. cooperi (19)

Tamiasciurus hudsonicus $(8,14,20)$

T. h. columbiensis (7)

T. h. gymnicus (16)

T. h. hudsonicus $(7,14)$

$T$. h. laurentianus (4)

Tamiasciurus spp. (20)

Tamias striatus $(7,20)$

T. s. griseus (20)

Zapus hudsonius $(8,16,18,20)$

Z. princeps (20)

Z. p. minor (7)

Zapus spp. $(7,20)$

Unspecified (2)

Dist.: Alta, BC, Man, NB, Nfld, NS, NWT,

Ont, Que, Sask, YT

Records: 1. Rothschild 1905 (Alta); 2. Jordan 1929 (BC), 3. 1932 (BC); 4. Eidmann 1935

(Que); 5. Wagner 1936 (BC); 6. Brown 1944

(Alta); 7. Holland 1949 (Alta, BC, NWT, Ont);

8. Hubbard 1949 (Ont); 9. Judd 1955 (NWT);

10. Harper 1956 (NWT); 11. Gabbutt 1961

(Nfld); 12. Harper 1961 (Nfld, Que); 13. Robert

1962 (Que); 14. Buckner 1964 (Man); 15.

Wilson 1967 (Ont); 16. Brown 1968 (NB); 17.

Buckner and Blasko 1969 (Man); 18. Wright

1979 (NS); 19. Whitaker and French 1982

(NB); 20. Holland 1984 (Alta, BC, Man, NB,

Nfld, NS, NWT, Ont, Que, Sask, YT)

Remarks: Rothschild (1905) and Brown (1944)

reported the hosts as Evotomys gapperi and $E$. saturatus. Holland (1984) felt that both were probably Clethrionomys gapperi loringi.

Monopsyllus ciliatus protinus (Jordan, 1929)

Hosts: Eutamias amoenus $(3,5)$
E. a. felix (3)
E. townsendii $(3,5)$
E. t. cooperi $(1,2)$
Eutamias spp. (5)
Glaucomys sabrinus (3)
G. s. oregonensis (4)
Lepus americanus $(3,5)$
Marmota caligata (5)
Martes americana caurina (3)
Microtus oregoni serpens (5)
Mustela erminea anguinae (3)
M. e. fallenda (3)
Ochotona princeps (5)
Ochotona spp. (5)
Peromyscus maniculatus $(3,5)$
P. m. austerus (3)
P. m. oreas (3)
Peromyscus spp. (5)

Procyon spp. (5)

Spilogale putorius gracilis (3)

S. p. latifrons (5)

S. p. phenax (5)

Sylvilagus floridanus (5)

Tamiasciurus douglasii (5)

T. d. mollipilosus $(2,3)$

T. hudsonicus $(1,5)$

T. h. lanuginosus $(3,5)$

T. h. picatus (5)

Tamiasciurus spp. $(3,5)$

Field mouse (5)

Red squirrel (5)

Squirrel (5)

Weasel (5)

Dist.: BC

Records: 1. Jordan 1929: 2. Wagner 1936a; 3.

Holland 1949; 4. Spencer 1956; 5. Holland 1984

Monopsyllus eumolpi (Rothschild, 1905)

Syn.: Ceratophyllus eumolpi Rothschild, 1905

Hosts: Clethrionomys gapperi (4)

Eutamias amoenus affinis $(1,2,4)$

E. a. ludibundus (4)

E. a. luteiventris (4)

E. minimus borealis $(1,4)$

E. m. selkirki (4)

Eutamias spp. $(1,4)$

Mustela frenata (4)

Ochotona princeps fenisex (4)

Spermophilus c. columbianus $(3,4)$

Tamiasciurus hudsonicus (4)

T. h. streatori $(2,4)$

Zapus princeps minor (4)

Dist.: Alta, BC, Ont, Sask

Records: 1. Rothschild 1905 (Alta, BC); 2.

Wagner 1936a (BC); 3. Holland 1944 (BC), 4.

1949 (Alta, BC, Ont, Sask)

Monopsyllus eumolpi eumolpi (Rothschild, 1905)

Syn.: Ceratophyllus eumolpi eumolpi

(Rothschild, 1905)

Monopsyllus eumolpi canadensis

Hubbard, 1949

Trichopsylla eumolpi eumolpi

(Rothschild, 1905)

Hosts: Eutamias amoenus (7)

E. a. affinis (7)

E. a. luteiventris (7)

E. minimus $(3,5,6,7)$

E. $m$. borealis (7)

E. m. minimus (7)

Eutamias spp. $(2,7)$

Glaucomys sabrinus (7)

Microtus oeconomus macfarlani (7)

M. pennsylvanicus drummondii (1)

Neotoma cinerea (7)

Neotoma spp. (7)

Ochotona princeps brooksi (7)

Ochotona spp. (7)

Peromyscus maniculatus (7)

P. m. artemisiae (7) 
Spermophilus columbianus (7)

Tamiasciurus hudsonicus $(3,7)$

Tamiasciurus spp. (7)

Tamias striatus $(3,7)$

Chipmunk $(4,7)$

Red squirrel (7)

Dist.: Alta, BC, Man, NWT, Ont, Sask, YT

Records: 1. Jordan 1932 (BC); 2. Brown 1944

(Alta); 3. Hubbard 1949a (Ont), 4. 1949b (Ont);

5. Buckner 1964 (Man); 6. Wilson 1967 (Ont);

7. Holland 1984 (Alta, BC, Man, NWT, Ont,

Sask, YT)

Remarks: Microtus oeconomus macfarlani is given by Holland (1984) as $M$. oregoni macfarlani. The geographical record suggests $M$. oeconomus macfarlani is the proper classification.

Monopsyllus thambus (Jordan, 1929)

Syn.: Ceratophyllus bakeri Wagner, 1934

Ceratophyllus thambus Jordan, 1929

Hosts: Clethrionomys gapperi (6)

C. rutilus (6)

Lynx canadensis (3)

Lynx spp. (1)

Microtus spp. (6)

Peromyscus maniculatus $(5,6)$

$P$. $m$. algidus (6)

P. m. borealis (6)

House mouse (2)

Mouse $(4,6)$

Dist.: Alta, BC, Nfld, NWT, Que, Sask, YT

Records: 1. Jordan 1929 (Alta); 2. Wagner 1933

(Nfld); 3. Brown 1944 (Alta); 4. Holland 1944

(NWT), 5. 1949 (NWT, Sask), 6. 1984 (Alta,

BC, Nfld, NWT, Que, YT)

Monopsyllus tolli (Wagner, 1901)

Hosts: Eutamias minimus borealis (2)

Dist.: YT

$$
\text { Ochotona collaris }(1,2)
$$

Records: 1. Holland 1952a, 2. 1984

Monopsyllus vison (Baker, 1904)

Syn.: Ceratophyllus vison (Baker, 1904)

Trichopsylla vison Baker, 1904

Hosts: Clethrionomys gapperi $(15,16)$

C. g. loringi (16)

C. rutilus (16)

C. r. dawsoni (16)

Clethrionomys spp. (16)

Eutamias minimus borealis (16)

Eutamias spp. (3)

Glaucomys sabrinus (16)

Glaucomys spp. (16)

Lepus americanus (8)

Lepus spp. (16)

Lynx canadensis (11)

Martes americana $(7,16)$

M. a. americana (14)

Mephitis mephitis (16)

Microtus longicaudus (16)
M. pennsylvanicus (16)

Microtus spp. (16)

Mustela erminea $(12,16)$

M. e. $\operatorname{arctica}(16)$

M. e. cicognanii (16)

M. e. invicta (16)

M. frenata (2)

M. vison (16)

$M$. v. energumenos (16)

Mustela spp. (16)

Peromyscus leucopus (15)

$P$. maniculatus $(15,16)$

Peromyscus spp. (16)

Procyon lotor $(15,16)$

Sciurus carolinensis (16)

Spermophilus c. columbianus (16)

$S$. parryii plesius (16)

Spermophilus spp. (16)

Tamiasciurus hudsonicus $(3,4,5,6,8,9$,

$10,13,15,16)$

T. h. baileyi (16)

T. h. columbiensis (16)

T. h. gymnicus (5)

T. h. hudsonicus (16)

T. h. minnesota (16)

T. h. picatus (16)

T. h. preblei (16)

T. h. richardsoni (16)

T. h. streatori $(2,16)$

T. h. ungavensis (16)

Tamiasciurus spp. (16)

Tamias striatus (8)

Vulpes vulpes (16)

Red squirrel (16)

Short-tailed weasel (16)

Squirrel $(5,16)$

Weasel (16)

Unspecified (1)

Dist.: Alta, BC, Man, Nfld, NB, NS, NWT,

Ont, Que, Sask, YT

Records: 1. Jordan 1929 (Alta, BC); 2. Wagner 1936a (BC); 3. Brown 1944 (Alta); 4. Hubbard 1949 (Ont); 5. Brown 1955 (NB); 6. Judd 1955 (NWT); 7. DeVos 1957 (Ont); 8. Scholten et al. 1962 (Ont); 9. Buckner 1964 (Man); 10.

Mitchell and Behin 1965 (NWT); 11. van Zyll

De Jong 1966 (Alta); 12. Wilson 1967 (Ont); 13.

Mahrt and Chai 1972 (Alta); 14. Holland 1979

(Ont); 15. Wright 1979 (NS); 16. Holland 1984

(Alta, BC, Man, Nfld, NS, NWT, Ont, Que,

Sask, YT)

Monopsyllus wagneri ophidius (Jordan)

Hosts: Microtus oregoni serpens

Microtus spp.

Peromyscus maniculatus

P. m. austerus

P. m. oreas

Peromyscus spp.

Tamiasciurus douglasii

Dist.: BC

Record: Holland 1984 
Monopsyllus wagneri systaltus (Jordan)

Hosts: Blarina brevicauda manitobensis (3)

Clethrionomys gapperi $(2,4)$

C. g. loringi (4)

Clethrionomys spp. (4)

Dipodomys ordii (4)

Lagurus curtatus (4)

Microtus ochrogaster (4)

M. pennsylvanicus (4)

Microtus spp. (4)

Mus musculus (4)

Peromyscus maniculatus $(2,4)$

P. $m$. bairdii (4)

P. m. nebrascensis (4)

Peromyscus spp. $(1,4)$

Sorex arcticus $(2,4)$

$S$. cinereus $(2,4)$

Zapus spp. (4)

House mouse (4)

Dist.: Alta, Man, Ont, Sask

Records: 1. Brown 1944 (Alta); 2 Buckner 1964

(Man); 3, Buckner and Blasko 1969 (Man); 4.

Holland 1984 (Alta, Man, Ont, Sask)

Monopsyllus wagneri wagneri (Baker, 1904)

Hosts: Eutamias spp. (2)

Microtus spp. (2)

Mustela frenata arizonensis (1)

Neotoma cinerea drummondii (2)

Neotoma spp. (2)

Peromyscus maniculatus (2)

P. m. alpinus (2)

$P$. m. artemisiae (2)

P. $m$. borealis (2)

P. m. maniculatus (2)

Permoyscus spp. $(1,2)$

Synaptomys borealis (2)

Synaptomys spp. (2)

Tamiasciurus hudsonicus (2)

Thomomys talpoides (2)

Zapus spp. (2)

Mice (2)

Rabbit (2)

Weasel (2)

Dist.: Alta, BC

Records: 1. Wagener 1936a (BC); 2. Holland 1984 (Alta, BC)

Nosopsyllus fasciatus (Bosc, 1801) Jordan, 1933

Syn.: Ceratophyllus canadensis Baker, 1904

Ceratophyllus fasciatus (Bosc, 1801)

Pulex fasciatus Bosc, 1801

Hosts: Blarina brevicauda (11)

Clethrionomys gapperi (11)

Microtus p. pennsylvanicus (6)

$M$. townsendii tetramerus (11)

Mus musculus (5)

Peromyscus maniculatus (7)

Rattus norvegicus $(2,3,4,5,7,8,9$,

$10,11)$

$R$. rattus alexandrinus $(5)$

$R$.r. rattus (5)
Rattus spp. $(7,11)$

Sorex spp. (7)

Tamiasciurus douglasii mollipilosus (7)

Mice (7)

Unspecified (1)

Dist.: BC, Man, NB, Nfld, NS, Ont, Que, Sask

Records: 1. Baker 1904 (Ont); 2. Spencer 1937

(BC); 3. 1939b (BC); 4. Holland 1940c (BC), 5.

$194 \mathrm{lb}$ (BC); 6. Baker 1946 (Que); 7. Holland

1949 (BC, Ont, Que, Sask); 8. Judd 1950 (Ont);

9. Brown 1968 (NB); 10. Wright 1979 (NS); 11.

Holland 1984 (BC, Man, Nfld, NS, Ont, Que)

Opisocrostis bruneri (Baker, 1895)

Syn.: Ceratophyllus bruneri (Baker, 1904)

Ceratophyllus saundersi (Jordan, 1933)

Oropsylla bruneri (Baker, 1904)

Pulex bruneri Baker, 1895

Hosts: Eutamias minimus borealis (5)

Marmota monax (6)

Microtus pennsylvanicus (10)

Spermophilus columbianus (3)

S. fránklinii $(2,5,6,9,10)$

$S$. richardsonii $(1,2,4,5,6,7,10)$

$S$. r. richardsonii (10)

S. tridecemlineatus $(2,3,4,5,6,8,10)$

S. $t$. tridecemlineatus $(4,7,10)$

Tamiasciurus hudsonicus (8)

T. h. hudsonicus $(7,10)$

Taxidea taxus $(5,10)$

Bush rabbit (5)

Dist.: Alta, Man, Sask

Records: 1. Jordan 1933 (Sask); 2. McLeod 1933 (Man); 3. Holland 1943 (Alta); 4. Brown 1944 (Alta); 5. Holland 1944 (unspecified), 6.

1949 (Alta, Man, Sask), 7. 1952a (Sask); 8.

Buckner 1964 (Man); 9. Hilton and Mahrt 1971

(Alta); 10. Holland 1984 (Alta, Man, Sask)

Opisocrostis labis (Jordan and Rothschild, 1922)

Syn.: Ceratophyllus labis Jordan and

Rothschild, 1922

Hosts: Cynomys ludovicianus (7)

C. l. ludovicianus (5)

Mustela frenata longicauda $(1,3,5)$

Spermophilus franklinii (5)

$S$. richardsonii $(2,4,5,6,7)$

S. r. richardsonii (7)

Taxidea t. taxus (5)

Ground squirrels (2)

Dist.: Alta, Sask

Records: 1. Jordan and Rothschild 1922 (Alta);

2. Gibbons and Humphreys 1941 (Alta); 3.

Brown 1944 (Alta); 4. Holland 1944 (Alta,

Sask), 5. 1949 (Alta, Sask); 6. Hilton and

Mahrt 1971 (Alta); 7. Holland 1984 (Alta, Sask)

Opisocrostis tuberculatus tuberculatus (Baker, 1904)

Syn.: Ceratophyllus tuberculatus Baker, 1904

Oropsylla tuberculata tuberculata (Baker, 1904) 
Hosts: Cynomys l. ludovicianus (5)

Lepus spp. (3)

Microtus spp. (7)

Ochotona collaris (7)

Spermophilus columbianus $(3,6,7)$

S. c. columbianus $(1,4,5)$

S. franklinii (4)

S. parryii (7)

S. richardsonii $(3,5,6,7)$

S. $r$. richardsonii $(2,4,7)$

S. tridecemlineatus (5)

S. t. tridecemlineatus (4)

Tamiasciurus hudsonicus (7)

Dist.: Alta, BC, Sask, YT

Records: 1. Holland 1940c (Alta); 2. Gibbons and Humphreys 1941 (Alta); 3. Brown 1944 (Alta); 4. Holland 1944 (Alta, BC, Sask), 5. 1949 (Alta, BC, Sask); 6. Hilton and Mahrt 1971 (Alta); 7. Holland 1984 (Alta, BC, Sask, YT)

Opisodasys keeni keeni (Baker, 1896)

Syn.: Pulex keeni Baker, 1896

Hosts: Clethrionomys gapperi (4)

Clethrionomys spp. $(3,4)$

Eutamias spp. (4)

Microtus longicaudus (4)

$M$. oregoni serpens $(3,4)$

M. townsendii (4)

M. t. townsendii (3)

Microtus spp. (3)

Neotoma cinerea (4)

Peromyscus maniculatus $(2,3)$

P. m. alpinus $(3,4)$

P. m. artemisiae $(3,4)$

$P$. m. austerus $(3,4)$

P. m. borealis (3)

P. $m$. interdictus (3)

P. m. keeni $(1,3)$

P. $m$. oreas $(3,4)$

$P$. sitkensis (4)

Peromyscus spp. $(3,4)$

Zapus spp. (3)

Chipmunk (4)

Weasel (3)

Dist.: Alta, BC

Records: 1. Baker 1896 (BC); 2. Wagner 1936a

(BC); 3. Holland 1949 (Alta, BC), 4. 1984 (Alta, BC)

Opisodasys pseudarctomys (Baker, 1904)

Syn.: Ceratophyllus pseudarctomys Baker, 1904

Hosts: Clethrionomys gapperi (3)

Glaucomys sabrinus $(1,3,5,6,9,10)$

G. s. alpinus (2)

G. s. canescens $(7,10)$

G. s. columbiensis $(3,10)$

G. s. macrotis $(3,8)$

G. s. makkovikensis (4)

G. volans (10)

Glaucomys spp. $(3,10)$
Lynx rufus (10)

Microtus pennsylvanicus (10)

Mustela frenata noveboracensis (10)

Tamiasciurus hudsonicus $(3,5,6,10)$

T. h. streatori (3)

Dist.: Alta, BC, Man, NB, Nfld, NS, Ont, Que Records: 1. Rothschild 1905 (BC); 2. Holland 1941a (BC), 3. 1949 (Alta, Ont); 4. Harper 1961 (Nfld); 5. Robert 1962 (Que); 6. Scholten et al. 1962 (Ont); 7. Buckner 1964 (Man); 8. Brown 1968 (NB); 9. Wright 1979 (NS); 10. Holland 1984 (Alta, BC, Man, NS, Ont, Que)

Remarks: Holland (1984) used Lynx fasciatus pallidus instead of Lynx rufus.

Opisodasys vesperalis (Jordan, 1929)

Syn.: Ceratophyllus vesperalis Jordan, 1929

Hosts: Glaucomys sabrinus $(3,4,6)$

G. s. alpinus $(2,4)$

G. s. columbiensis $(1,4,6)$

G. s. fuliginosus (4)

G. s. oregonensis $(5,6)$

Lepus americanus columbiensis (6)

Lynx canadensis (4)

Tamiasciurus douglasii (6)

Weasel (4)

Dist.: BC

Records: 1. Jordan 1929; 2. Wagner 1940; 3. Holland 1941, 4. 1949; 5. Spencer 1956; 6.

Holland 1984

Remarks: According to Holland (1984), Wagner (1940) identified the specimen as Opisodasys pseudarctomys by mistake.

Orchopeas caedens (Jordan, 1925)

Hosts: Martes americana (1)

Tamiasciurus hudsonicus $(2,3)$

Dist.: Alta, NWT, Ont

Remarks: 1. Brown 1944 (Alta); 2. Judd 1955

(NWT); 3. Scholten et al. 1962 (Ont)

Orchopeas caedens caedens (Jordan, 1925)

Syn.: Ceratophyllus caedens (Jordan, 1925)

Hosts: Eutamias spp. (6)

Glaucomys sabrinus $(4,6)$

Glaucomys spp. (6)

Martes americana $(1,6)$

M. a. actuosa (6)

Mustela erminea (6)

M. e. cicognanii (6)

M. e. invicta (6)

M. e. richardsonii (6)

$M$. frenata longicauda (6)

M. vison $(2,6)$

Peromyscus maniculatus (6)

Spermophilus franklinii (2)

S. parryii (6)

Tamiasciurus hudsonicus $(2,3,4,5,6)$

T. h. preblei (2)

Tamiasciurus spp. (6)

Vulpes vulpes (2)

Pine marten (6) 
Red squirrel (6)

Tree squirrel (6)

Weasel $(2,6)$

Dist.: Alta, BC, Man, Nfld, NWT, Que, Sask, YT

Records: 1. Jordan 1925 (Alta); 2. Holland 1949 (Alta, BC, NWT, Sask, YT); 3. Gabbutt

1961 (Nfld); 4. Buckner 1964 (Man); 5. Mahrt and Chai (Alta); 6. Holland 1984 (Alta, BC, Man, Que, YT)

Orchopeas caedens durus (Jordan, 1929)

Syn.: Ceratophyllus caedens durus Jordan, 1929

Hosts: Clethrionomys gapperi (12)

Clethrionomys spp. (15)

Eutamias amoenus (4)

Eutamias spp. (6)

Glaucomys sabrinus $(6,14,15)$

Glaucomys spp. (15)

Lynx canadensis (6)

L. rufus (15)

Martes americana $(6,15)$

M. a. actuosa (15)

M. a. americana (13)

Mephitis m. mephitis (6)

Mustela erminea $(6,11,15)$

M. e. cicognanii (15)

M. e. richardsonii (12)

$M$. frenata (1)

M. f. arizonensis $(5,15)$

$M$. f. nevadensis (4)

M. vison (7)

Mustela spp. $(1,6,15)$

Peromyscus maniculatus (14)

P. m. abietorum (12)

Sciurus carolinensis $(12,15)$

Tamiasciurus douglasii (15)

T. d. mollipilosus $(4,15)$

T. hudsonicus $(1,2,6,14)$

T. h. columbiensis (6)

T. h. gymnicus $(8,12)$

T. h. hudsonicus $(6,15)$

T. h. preblei (9)

T. h. streatori $(1,4,6,15)$

T. h. ungavensis (10)

Tamiasciurus spp. (15)

Tamias striatus (15)

Vulpes vulpes (15)

Chipmunk (15)

Red squirrel (3)

Squirrel (15)

Weasel $(3,15)$

Dist.: Alta, BC, NB, Nfld, NS, NWT, Ont,

Que, Sask

Records: 1. Jordan 1929 (BC), 2. 1932 (BC); 3.

Spencer 1936 (BC); 4. Wagner 1936 (BC); 5.

Holland 1938 (BC), 6. 1949 (BC, Nfld, NWT);

7. judd 1954a (Ont); 8. Brown 1955 (NB); 9.

Harper 1956 (NWT), 10. 1961 (Nfld, Que); 11. Wilson 1967 (Ont); 12. Brown 1968 (NB); 13.

Holland 1979 (Ont); 14. Wright 1979 (NS); 15.
Holland 1984 (Alta, BC, NB, Nfld, NWT, Ont, Que, Sask)

Remarks: Wagner's (1936) record from $T . d$. mollipilosus is possibly a misidentification (Holland 1984).

Orchopeas howardi howardi (Baker, 1895)

Syn.: Orchopeas wickhami (Baker, 1895)

Hosts: Glaucomys volans (5)

G. v. volans $(1,3,5)$

Sciurus carolinensis (5)

S. niger (5)

Sciurus spp. (5)

Tamiasciurus hudsonicus $(4,5)$

T. h. gymnicus (2)

T. h. loquax (1)

Tamias striatus lysteri (1)

Dist.: Alta, Ont, Que

Records: 1. Jameson 1943 (Ont); 2. Baker 1944

(Que); 3. Holland 1949 (Ont); 4. Hubbard

1949a (Ont); 5. Holland 1984 (Alta, Ont)

Orchopeas leucopus (Baker, 1904)

Syn.: Ceratophyllus leucopus Baker, 1904

Hosts: Blarina brevicauda (6)

B. b. talpoides (4)

Blarina spp. (19)

Clethrionomys gapperi $(4,12,19)$

C. g. gapperi (4)

C. g. loringi $(1,3,14,19)$

C. g. ochraceus (10)

C. rutilus (19)

Clethrionomys spp. (19)

Glaucomys sabrinus macrotis (4)

G. volans (19)

G. v. volans $(19)$

Glaucomys spp. (19)

Microtus c. chrotorhinus (4)

M. pennsylvanicus $(4,12,19)$

M. p. drummondii $(14,19)$

M. p. pennsylvanicus (4)

Microtus spp. $(4,19)$

Mus musculus $(4,19)$

Napaeozapus insignis (12)

Ondatra z. zibethicus (4)

P. leucopus $(17,19)$

P. l. noveboracensis $(2,4)$

P. maniculatus $(4,5,12,13,14,15$,

$18,19)$

P. m. abietorum (10)

P. m. algidus (19)

P. m. bairdii $(4,12,19)$

P. $m$. borealis $(1,3,4,11,19)$

P. m. gracilis (4)

P. m. nebracensis (4)

Peromyscus spp. $(4,7,8,9,16,19)$

Rattus spp. (19)

Sorex cinereus (19)

S. c. cinereus (14)

Synaptomys spp. $(12,19)$

Tamiasciurus hudsonicus (12)

T. h. hudsonicus $(12,16)$ 
Tamias striatus $(4,12)$

Tamias spp. (19)

Mice (4)

Dist.: Alta, BC, Man, NB, Nfld, NS, NWT,

Ont, Que, Sask, YT

Records: 1. Rothschild 1905 (Alta); 2. Jameson

1943 (Ont); 3. Brown 1944 (Alta); 4. Holland

1949 (Alta, BC, Man, NWT, Ont, Que, Sask,

YT); 5. Hubbard 1949a (Ont); 6. Jameson 1950

(Ont); 7. Judd 1950 (Ont), 8. 1953 (Ont), 9.

1954a (Ont); 10. Brown 1955 (NB); 11. Judd

1955 (NWT); 12. Robert 1962 (Que); 13.

Scholten et al. 1962 (Ont); 14. Buckner 1964

(Man); 15. Brown 1968 (NB); 16. Buckner and

Blasko 1969 (Man); 17. Wright 1979 (NS); 18.

Whitaker and French 1982 (NB); 19. Holland

1984 (Alta, BC, Man, NB, Nfld, NS, NWT,

Ont, Que, Sask)

Remarks: Brown (1944) and Rothschild (1905)

reported their hosts as Evotomys gapperi

saturatus and Evotomys saturatus, respectively. Holland (1984) reported Peromyscus sp. and $P$. arcticus. The validity of $P$. arcticus is uncertain so we have included both under Peromyscus spp.

Orchopeas nepos (Rothschild, 1905)

Hosts: Glaucomys sabrinus oregonensis (3)

Mustela erminea fallenda (3)

$M$. vison energumenos (3)

Sciurus carolinensis (4)

Spilogale putorius $(1,3)$

Tamiasciurus douglasii (4)

T. d. mollipilosus $(2,3)$

Tamiasciurus spp. (4)

Dist.: BC

Weasel $(3,4)$

Records: 1. Rothschild 1905; 2. Wagner 1936a;

3. Holland 1949, 4. 1984

Remarks: Holland (1949) records Spilogale putorius as $S$. gracilis olympica.

Orchopeas sexdentatus agilis (Rothschild, 1905)

Syn.: Ceratophyllus agilis Rothschild, 1905

Hosts: Clethrionomys gapperi (7)

C. g. saturatus (5)

Eutamias minimus (7)

Lepus americanus columbiensis (7)

Lynx spp. (7)

Martes americana (7)

Microtus longicaudus (5)

Mustela frenata $(1,5,7)$

M. f. arizonensis (7)

M. f. longicauda (4)

M. f. nevadensis $(2,7)$

$M$. vison energumenos (7)

Neotoma cinerea $(1,4,6,7)$

N. c. cinerea $(7)$

N. c. drummondii (7)

N. c. occidentalis $(2,3,5,7)$

Neotoma spp. (7)

Ochotona princeps $(1,2,4,5,7)$
O. p. brooksi (7)

Ochotona spp. (7)

Peromyscus spp. (7)

Sciurus spp. (7)

Spilogale putorius (5)

Sylvilagus $n$. nuttallii (5)

Tamiasciurus hudsonicus (7)

T. h. preblei $(1,7)$

T. h. richardsoni (4)

Skunk (7)

Weasel $(5,7)$

Wood rat (7)

Dist.: Alta, BC, YT

Records: 1. Rothschild 1905 (Alta, BC); 2.

Wagner 1936 (BC); 3. Holland 1938 (BC); 4.

Brown 1944 (Alta); 5. Holland 1949 (BC), 6.

1952b (Alta), 7. 1984 (Alta, BC, YT)

Remarks: Holland (1949) records Spilogale putorius as $S$. gracilis olympica. Holland's (1952) specimens were taken from the nest of $N$. cinerea. Holland (1984) records four hosts: Mustela frenata arizonensis $(=M$. arizonensis) and Neotoma cinerea occidentalis $(=N$. $c$. saxamans).

Oropsylla alaskensis (Baker, 1904)

Syn.: Ceratophyllus alaskensis Baker, 1904

Hosts: Alopex lagopus (4)

A. l. lagopus $(2,4)$

Dicrostonyx spp. (4)

Lynx canadensis (4)

Mustela erminea arctica (2)

Spermophilus parryii (4)

S. p. parryii $(1,3)$

Spermophilus spp. $(1,4)$

Ground squirrel (4)

Dist.: NWT, YT

Records: 1. Holland 1944 (NWT); 2. Jellison 1945 (NWT); 3. Holland 1949 (NWT), 4. 1984 (NWT, YT)

Oropsylla arctomys (Baker, 1904)

Syn.: Atheopsylla septentrionalis Stewart and Holland, 1940

Ceratophyllus arctomys Baker, 1904

Hosts: Alopex l. lagopus (17)

Canis latrans $(5,17)$

Glaucomys volans (17)

Marmota caligata (6)

M. c. okanagona (5)

M. $\operatorname{monax}(6,10,13,16,17)$

M. m. canadensis $(11,12,17)$

M. m. petrensis $(1,2,3,5)$

$M$. $m$. rufescens $(6,8,17)$

Marmota spp. $(5,7,17)$

Martes americana (17)

M. a. americana (15)

M. pennanti (17)

M. p. pennanti $(6,17)$

Mephitis m. mephitis (6)

Mephitis spp. (17)

Peromyscus maniculatus (17) 
Spermophilus columbianus (17)

S. franklinii (17)

Spermophilus richardsonii (4)

Sylvilagus spp. (6)

Tamiasciurus hudsonicus (17)

Taxidea t. taxus (6)

Ursus americanus (14)

Vulpes vulpes $(6,9,11,17)$

Bear (6)

Dist.: Alta, BC, Man, NB, NS, NWT, Ont,

Que, Sask

Records: 1. Wagner 1936 (BC); 2. Stewart and Holland 1940 (BC); 3. Holland 1949a (BC); 4. Brown 1944 (Alta); 5. Jellison 1945 (BC, Ont, Sask); 6. Holland 1949 (Alta, BC, Man, NB, Ont, Que, Sask); 7. Hubbard 1949 (Ont); 8. Judd 1950 (Ont), 9. 1953 (Ont); 10. Scholten et al. 1962 (Ont); 11. Buckner 1964 (Man); 12. Brown 1968 (NB); 13. Ko 1972b (Ont); 14. Addison et al. 1978 (Ont); 15. Holland 1979 (Ont); 16. Wright 1979 (NS); 17. Holland 1984 (Alta, BC, Man, NB, NWT, Ont, Que, Sask)

Remarks: Holland (1984) recorded the host as Spermophilus columbianus albertae. The validity of $S$. c. albertae is uncertain so we included it under $S$. columbianus.

Oropsylla idahoensis (Baker, 1904)

Syn.: Ceratophyllus idahoensis Baker, 1904 Ceratophyllus poeantis Rothschild, 1905

Hosts: Eutamias townsendii (8)

Eutamias spp. (8)

Marmota flaviventris avara (8)

Marmota spp. (1)

Microtus longicaudus (6)

Mustela frenata (8)

M. f. longicauda (1)

M.f. oribasus (6)

Mustela spp. (8)

Ochotona collaris $(8)$

O. princeps (8)

O.p.princeps (6)

Spermophiluscolumbianus $(1,2,3,5,6,7,8)$

S. c. columbianus $(4,6,8)$

S. lateralis $(5,8)$

S. l. tescorum $(1,3,6)$

S. parryii (8)

S. p. plesius (8)

S. richardsonii (7)

S. r. richardsonii (6)

S. saturatus $(6,8)$

S. tridecemlineatus (6)

Spermophilus spp. $(4,8)$

Thomomys talpoides andersoni (6)

Thomomys spp. (8)

Dist.: Alta, BC, YT

Records: 1. Rothschild 1905 (Alta, BC); 2. Wagner 1936a (BC); 3. Brown 1944 (Alta); 4. Holland 1944 (Alta, BC); 5. Jellison 1945 (Alta, BC); 6. Holland 1949 (Alta, BC); 7. Hilton and Mahrt 1971 (Alta); 8. Holland 1984 (Alta, BC, YT)
Oropsylla rupestris (Jordan, 1929)

Hosts: Canis spp. (1)

Cynomys l. ludovicianus (7)

Lepus townsendii campanius (7)

Mustela frenata longicauda $(1,4,7)$

Mustela spp. $(2,6)$

Peromyscus spp. (9)

Rattus norvegicus $(5,9)$

Spermophilus franklinii (7)

S. parryii (9)

S. richardsonii $(1,2,4,6,7,8,9)$

S. r. richardsonii $(3,5,9)$

Spermophilus spp. (5)

Taxidea taxus (4)

Thomomys spp. $(1,2,4)$

Weasel (9)

Dist.: Alta, Man, Sask, YT

Records: 1. Jordan 1929 (Alta); 2. Wagner 1936a (Alta); 3. Gibbons and Humphreys 1941 (Alta); 4. Brown 1944 (Alta); 5. Holland 1944 (Sask); 6. Jellison 1945 (Alta, Sask); 7. Holland 1949 (Alta, Man, Sask); 8. Hilton and Mahrt 1971 (Alta); 9. Holland 1984 (Alta, Man, Sask, YT)

Remarks: Holland (1984) recorded both Spermophilus parryii and S. undulatus.

Tarsopsylla octodecimdentata coloradensis (Baker, 1895)

Syn.: Ceratophyllus coloradensis Baker, 1904 Pulex coloradensis Baker, 1895

Tarsopsylla coloradensis Jordan, 1933

Hosts: Glaucomys sabrinus $(2,5)$

G. s. alpinus (1)

Lynx rufus (5)

Martes americana $(2,5)$

M. a. americana (4)

Tamiasciurus hudsonicus $(2,3,5)$

Tamiasciurus spp. (5)

Squirrel (5)

Dist.: Alta, BC, Man, NWT, Ont, Sask, YT

Records: 1. Holland 1941a (BC), 2. 1949 (Alta, BC, NWT); 3. Mahrt and Chai 1972 (Alta); 4. Holland 1979 (Ont), 5. 1984 (Alta, BC, Man, NWT, Ont, Sask, YT)

Thrassis acamantis (Rothschild, 1905)

Syn.: Ceratophyllus acamantis Rothschild, 1905

Syn.: Oropsylla acamantis

Hosts: Canis latrans (1)

Eutamias spp. (5)

Marmota flaviventris $(2,8)$

M. f. avara $(1,3,4,5,6,7,8)$

M. vancouverensis (3)

Marmota spp. $(5,8)$

Mephitis mephitis (1)

Mustela vison energumenos (1)

Peromyscus spp. (8)

Spermophilus c. columbianus (3)

Synaptomys spp. (8)

Tamiasciurus hudsonicus streatori (5) 
Chipmunk (8)

Groundhog (8)

Skunk (8)

Weasel (8)

Dist.: Alta, BC

Record: 1. Rothschild 1905 (BC); 2. Wagner 1936a (BC); 3. Spencer 1936 (BC); 4. Holland 1938 (BC); 5. Brown 1944 (Alta); 6. Holland 1944 (BC), 7. 1949 (BC), 8. 1984 (Alta, BC)

Remarks: Rothschild's (1905) specimens of $T$. acamantis from Canis latrans and Mustela vison ergumenos may possibly be Thrassis spenceri spenceri (Holland 1984).

Thrassis bacchi bacchi (Rothschild, 1905)

Syn.: Ceratophyllus bacchi Rothschild, 1905 Oropsylla bacchi

Thrassis gladiolis gladiolis (Jordan)

Hosts: Cynomys ludovicianus (5)

C. l. ludovicianus (4)

Mustela frenata longicauda (4)

Spermophilus franklinii $(3,5)$

S. richardsonii (5)

S. r. richardsonii $(3,4)$

S. tridecemlineatus $(1,2,4,5)$

S. $t$. tridecemlineatus (3)

Weasel (5)

Dist.: Alta, Man, Sask

Records: 1. Rothschild 1905 (Alta); 2. Brown 1944 (Alta); 3. Holland 1944 (Alta, Sask); 4. 1949 (Alta, Man, Sask), 5. 1984 (Alta, Man, Sask)

Thrassis petiolatus (Baker, 1904)

Syn.: Ceratophyllus petiolatus Baker, 1904

Hosts: Eutamias spp. (5)

Microtus pennsylvanicus (5)

Spermophilus columbianus $(2,4,5)$

S. c. columbianus (3)

S. lateralis (5)

$S$. richardsonii (5)

Spermophilus spp. (5)

Thomomys spp. (1)

Dist.: Alta, BC

Records: 1. Jordan 1929 (BC); 2. Brown 1944

(Alta); 3. Holland 1944 (BC), 4. 1949 (Alta,

BC), 5. 1984 (Alta, BC)

Thrassis spenceri spenceri Wagner, 1936

Syn.: Diamanus montanus Holland, 1941

Thrassis acamantis Spencer, 1936

Thrassis spenceri vancouverensis Stark, 1957

Hosts: Gulo luscus (3)

Marmota caligata $(4,6)$

M. c. cascadensis (4)

M. c. okanagana $(2,4,6)$

M. monax (6)

M. m. petrensis (4)

$M$. vancouverensis $(4,5,6)$

Marmota spp. $(1,4,6)$

Dist.: Alta, BC
Records: 1. Spencer 1936a (BC); 2. Wagner 1936a (BC); 3. Holland 1941a (BC), 4. 1949 (Alta, BC); 5. Stark 1957 (BC); 6. Holland 1984 (Alta, BC)

Family HYSTRICHOPSYLLIDAE

Anomiopsyllus montanus Collins, 1936

Host: Neotoma cinerea

Dist.: Alta

Records: Holland 1952, 1984

Atyphloceras bishopi Jordan, 1933

Syn.: Saphiopsylla bishopi (Jordan, 1933)

Hosts: Blarina brevicauda (3)

B. b. talpoides (2)

Clethrionomys gapperi (6)

C. g. loringi (5)

C. g. proteus $(4,6)$

Clethrionomys spp. (6)

Microtus chrotorrhinus (6)

M. pennsylvanicus $(4,6)$

M. p. pennsylvanicus $(1,2)$

Microtus spp. (6)

Peromyscus maniculatus gracilis (1)

Dist.: Man, Nfld, Ont, Que

Records: 1. Baker 1946 (Que); 2. Holland 1949

(Ont); 3. Jameson 1950 (Ont); 4. Hopkins and

Rothschild 1962 (Nfld, Ont, Que); 5. Buckner and Blasko 1969 (Man); 6. Holland 1984 (Man, Nfld, Ont, Que)

Atyphloceras multidentatus multidentatus (Fox,

Syn.: Atyphloceras artius Jordan, 1933

Hosts: Microtus oregoni serpens $(2,3)$

M. townsendii tetramerus (3)

Peromyscus maniculatus (2)

P. m. austerus (2)

Peromyscus spp. $(1,3)$

Rattus norvegicus (2)

Spilogale putorius gracilis (2)

Tamiasciurus hudsonicus streatori (2)

Dist.: BC

Tamiasciurus spp. (3)

Records: 1. Jordan 1933; 2. Holland 1949, 3. 1984

Callistopsyllus terinus campestris Holland, 1949

Syn.: Callistopsyllus campestris Holland, 1949

Hosts: Peromyscus maniculatus nebrascensis

(1)

Peromyscus spp. $(2,3)$

Dist.: Alta, Sask

Records: 1. Holland 1949 (Alta, Sask); 2.

Hopkins and Rothschild 1962 (Alta); 3.

Holland 1984 (Alta)

Callistopsyllus terinus terinus (Rothschild, 1905)

Syn.: Ceratophyllus terinus Rothschild, 1905

Hosts: Peromyscus maniculatus $(3,4,5,6)$

P. m. artemisiae (5)

Peromyscus spp. (7) 
Dist.: BC

Spermophilus columbianus $(1,2,7)$

Records: 1. Rothschild 1905; 2. Wagner 1936a, 3. 1940; 4. Holland 1941a; 5. Holland 1949; 6 .

Hopkins and Rothschild 1962; 7. Holland 1984

Catallagia borealis Ewing, 1929

Hosts: Blarina brevicauda $(1,2)$

Clethrionomys gapperi $(7,10)$

C. g. loringi $(8,10)$

C. g. ochraceus $(4,10)$

C. g. proteus $(3,5)$

Microtus chrotorrhinus (9)

M. pennsylvanicus $(5,10)$

Peromyscus maniculatus (6)

P. m. abietorum (4)

P. m. bairdii (10)

Dist.: Alta, Man, NB, Ont, Que

Records: 1. Brown 1944 (Alta); 2. Jameson 1950 (Ont); 3. Holland 1952a (Que); 4. Brown

1955 (NB); 5. Hopkins and Rothschild 1962

(Ont, Que); 6. Buckner 1964 (Man); 7. Brown

1968 (NB); 8. Buckner and Blasko 1969 (Man);

9. Whitaker and French 1982 (NB); 10.

Holland 1984 (Man, NB, Ont, Que)

Catallagia charlottensis (Baker, 1898)

Syn.: Ceratophyllus charlottensis (Baker, 1904)

Pulex charlottensis Baker, 1898

Typhlopsylla charlottensis Baker, 1898

Hosts: Clethrionomys gapperi $(5,7)$

C. g. cascadensis (7)

C. g. caurinus $(7)$

C. g. saturatus (4)

Clethrionomys spp. (7)

Eutamias spp. (7)

Microtus longicaudus macrurus (5)

$M$. oregoni serpens $(5,7)$

$M$. townsendii tetramerus (7)

M. t. townsendii (5)

Microtus spp. $(5,7)$

Neotoma cinerea $(2,4)$

Neurotrichus g. gibbsii (5)

Peromyscus leucopus $(2,4)$

$P$. maniculatus $(5,7)$

P. m. austerus $(5,7)$

P. $m$. borealis $(2,5)$

$P$. $m$. interdictus (5)

P. m. keeni (1)

P. m. macrorhinus (3)

P. m. oreas (5)

Peromyscus spp. $(2,7)$

Rattus norvegicus $(5,6)$

Scapanus orarius schefferi (5)

Scapanus spp. (5)

Sciurus spp. (7)

Spermophilus columbianus (3)

Synaptomys spp. $(5,7)$

Tamiasciurus hudsonicus (7)

Dist.: Alta, BC

Records: 1. Baker 1898 (BC); 2. Rothschild

1905 (Alta, BC); 3. Wagner 1936a (BC);
4. Brown 1944 (Alta); 5. Holland 1949 (BC); 6. Hopkins and Rothschild 1962 (BC); 7. Holland 1984 (BC)

Remarks: Rev. J.H. Keen collected the specimens from a mouse nest (Baker 1898). The host was probably P. m. keeni (Holland 1984).

Catallagia dacenkoi fulleri Holland, 1951

Hosts: Clethrionomys gapperi $(1,4)$

C. g. athabascae $(1,2,4)$

C. rutilus $(3,4)$

C. r. dawsoni $(1,4)$

Clethrionomys spp. (4)

Lemmus sibiricus trimucronatus (4)

Microtus longicaudus vellerosus (4)

M. oeconomus (4)

$M$. pennsylvanicus $(2,4)$

M. p. drummondii $(1,2,4)$

Microtus spp. $(1,2,4)$

Peromyscus spp. (4)

Dist.: Alta, BC, Man, NWT, YT

Records: 1. Holland 1951 (BC, Man, NWT); 2. Hopkins and Rothschild 1962 (NWT, YT); 3. Mitchell and Behin 1965 (NWT); 4. Holland 1984 (Alta, BC, Man, YT)

Catallagia decipiens Rothschild, 1915

Hosts: Clethrionomys gapperi (5)

C. g. loringi (1)

C. g. saturatus $(3,4)$

C. rutilus dawsoni (5)

Clethrionomys spp. (5)

Eutamias amoenus affinis $(4,5)$

Eutamias spp. (5)

Lagurus curtatus (5)

Lepus spp. (5)

Microtus longicaudus $(4,5)$

M. l. mordax (4)

$M$. montanus canescens $(4,5)$

M. pennsylvanicus (5)

M. p. drummondii (4)

Microtus spp. (5)

Mustela erminea (5)

M. frenata (4)

Neotoma cinerea $(1,5)$

Neotoma spp. (5)

Ochotona princeps (4)

Ochotona spp. (5)

Onychomys leucogaster missouriensis

(4)

Peromyscus maniculatus $(4,5)$

P. m. alpinus (5)

P. $m$. artemisiae $(4,5)$

P. $m$. borealis $(4,5)$

P. m. nebrascensis (4)

Peromyscus spp. $(1,5)$

Phenacomys intermedius $(4,5)$

Sorex spp. $(4,5)$

Spermophilus columbianus $(2,3,4,5)$

$S$. richardsonii $(4,5)$

Tamiasciurus hudsonicus $(4,5)$

Thomomys talpoides fuscus (5) 
T. t. incensus (4)

Zapus hudsonicus (5)

Z. princeps idahoensis (4)

Zapus spp. (5)

Gopher (5)

Vole (5)

Dist.: Alta, BC, Man, Sask, YT

Records: 1. Rothschild 1915 (Alta, BC); 2.

Wagner 1936 (BC); 3. Brown 1944 (Alta); 4.

Holland 1949 (Alta, BC, Sask), 5. 1984 (Alta,

BC, Man, Sask, YT)

Catallagia jellisoni Holland, 1954

Hosts: Clethrionomys gapperi $(1,2,3)$

C. rutilus dawsoni (3)

Microtus pennsylvanicus (3)

M. p. drummondii (2)

Neotoma cinerea $(2,3)$

Peromyscus maniculatus (3)

Dist.: Alta, BC, YT

Records: 1. Holland 1954 (Alta); 2. Hopkins and Rothschild 1962 (Alta, BC); 3. Holland 1984 (Alta, BC, YT)

Remarks: The host was either $C$. r. dawsoni or Lemmus sibiricus (Holland 1984).

Catallagia mathesoni Jameson, 1950

Host: Peromyscus maniculatus

Dist.: BC

Records: 1. Holland 1952a, 2. 1984

Catallagia sculleni chamberlini Hubbard, 1940

Hosts: Clethrionomys spp.

Microtus richardsoni

Microtus spp.

Dist.: BC

Peromyscus spp.

Record: Holland 1984

Catallagia sculleni sculleni Hubbard, 1940

Syn.: Catallagia chamberlini Hubbard, 1940

Hosts: Peromyscus maniculatus (1)

P. m. austerus (2)

Peromyscus spp. (2)

Dist.: BC

Scapanus orarius schefferi $(1,2)$

Records: 1. Holland 1949, 2. 1984

Catallagia spp.

Host: Peromyscus maniculatus

Dist.: Man

Record: Buckner 1964

Conorhinopsylla stanfordi Stewart, 1930

Hosts: Glaucomys sabrinus $(2,4)$

G. volans (3)

G. v. volans $(1,4)$

Sciurus carolinensis (4)

Dist.: Ont

Tamiasciurus spp. (4)

Records: 1. Holland 1949; 2. Hopkins and

Rothschild 1962; 3. Tipton et al. 1979;

4. Holland 1984
Corrodopsylla curvata curvata (Rothschild, 1915)

Syn.: Doratopsylla curvata Rothschild, 1915

Hosts: Blarina brevicauda $(5,10,13,14)$

B. b. angusta (12)

B. b. manitobensis (11)

B. b. talpoides (4)

Blarina spp. (14)

Clethrionomys gapperi $(10,14)$

C. rutilus (14)

C. r. dawsoni (14)

Clethrionomys spp. (14)

Microsorex hoyi $(10,14)$

Microsorex spp. (14)

Microtus oeconomus (14)

M. pennsylvanicus (14)

Microtus spp. (14)

Mustela erminea (14)

Peromyscus maniculatus (10)

Peromyscus spp. (14)

Sorex arcticus laricorum $(11,14)$

S. cinereus $(9,13,14)$

S. c. acadicus (6)

S. c. cinereus (14)

S. c. haydeni (4)

S. c. miscix (8)

S. fumeus $(13,14)$

S. palustris $(9,13,14)$

$S$. vagrans (4)

S. v. obscurus (14)

Sorex spp. $(1,2,3,4,7,14)$

Zapus hudsonicus $(3,13,14)$

Zapus spp. (1)

Dist.: Alta, BC, Man, NB, Nfld, NS, NWT,

Ont, Que, Sask, YT

Records: 1. Rothschild 1915 (Alta); 2. Wagner 1936 (BC); 3. Brown 1944 (Alta); 4. Holland 1949 (BC, Ont, Sask); 5. Hubbard 1949 (Ont); 6. Brown 1955 (NS); 7. Gabbutt 1961 (Nfld); 8 . Harper 1961 (Que); 9. Scholten et al. 1962 (Ont); 10. Robert 1962 (Que); 11. Buckner 1964 (Man); 12. Brown 1968 (NB); 13. Wright 1979 (NS); 14. Holland 1984 (Alta, BC, Man, NB, Nfld, NS, NWT, Ont, Que, Sask, YT)

Remarks: Holland (1949) recorded Sorex obscurus obscurus as the host for C. c. curvata. There is some doubt about the validity of $S$. $O$. obscurus so we recorded it as Sorex vagrans.

Corrodopsylla curvata obtusata (Wagner, 1929)

Syn.: Doratopsylla curvata obtusata Wagner, 1929

Hosts: Microtus oregoni serpens (3)

Neurotrichus g. gibbsii (3)

Sorex palustris (6)

$S$. vagrans vancouverensis (4)

Sorex spp. $(1,2,3,4,5,6)$

Dist.: BC

Records: 1. Wagner 1929, 2. 1936a; 3. Holland 1942, 4. 1949; 5. Hopkins and Rothschild 1966; 6. Holland 1984 
Corrodopsylla hamiltoni (Traub, 1944)

Syn.: Doratopsylla hamiltoni Traub, 1944

Host: Sorex cinereus

Dist.: NB

Record: Whitaker and French 1982

Corypsylla jordani Hubbard, 1940

Hosts: Neurotrichus g. gibbsii $(1,2,3)$

Neurotrichus spp. (3)

Dist.: BC

Records: 1. Holland 1949; 2. Hopkins and

Rothschild 1962; 3. Holland 1984

Corypsylla kohlsi Hubbard, 1940

Syn.: Corypsylloides kohlsi (Hubbard, 1940)

Hosts: Sorex vagrans (2)

S. v. obscurus (1)

S. o. setosus (2)

Sorex spp. (2)

Dist.: BC

Records: 1. Holland 1949, 2. 1984

Corypsylla ornata Fox, 1908

Hosts: Microtus oregoni serpens $(3,5)$

M. t. townsendii (3)

Neurotrichus spp. $(4,5)$

Scapanus orarius schefferi $(1,2,3,5)$

S. townsendii (3)

Scapanus spp. $(4,5)$

Dist.: BC

Mole (5)

Records: 1. Wagner 1940; 2. Holland 1941a, 3. 1949; 4. Hopkins and Rothschild 1962; 5.

Holland 1984

Ctenophthalmus pseudagyrtes pseudagyrtes Baker, 1895

Hosts: Blarina brevicauda $(6,7,10,13,14,15$,

16)

B. b. angustus (13)

B. b. pallida $(9)$

B. b. talpoides $(2,5,16)$

Blarina spp. (16)

Clethrionomys gapperi $(5,10,13,14,16)$

C. g. gaspeanus (13)

C. g. loringi $(1,12,16)$

C. g. ochraceus (9)

C. g. saturatus (3)

Clethrionomys spp. (16)

Condylura cristata $(13,14,15,16)$

C. c. cristata (5)

C. c. nigra (4)

Eutamias minimus $(5,6,16)$

E. m. borealis $(12,16)$

Eutamias spp. $(5,16)$

Glaucomys volans $(12,16)$

Microtus chrotorrhinus $(13,15)$

M. ochrogaster (16)

M. o. minor $(12,16)$

M. pennsylvanicus $(6,10,13,14,16)$

M. p. drummondii $(1,3,5,12)$

M. p. pennsylvanicus $(2,4,8)$
Microtus spp. (16)

Mustela spp. (16)

Napaeozapus insignis (14)

Napaeozapus spp. (16)

Parascalops breweri $(2,5,12,16)$

Parascalops spp. (16)

Peromyscus leucopus (14)

P. l. noveboracensis (5)

P. maniculatus $(6,10,15)$

P. m. abietorum (9)

P. m. anticostiensis (16)

P. m. bairdii $(11,16)$

P. m. borealis (5)

Peromyscus spp. $(5,16)$

Pitymys pinetorum scalapoides (2)

Rattus norvegicus $(10,13)$

Scalopus aquaticus (16)

Sorex cinereus $(5,16)$

S. fumeus $(15,16)$

Spermophilus $r$. richardsonii (5)

Synaptomys borealis (15)

S. cooperi (15)

Tamiasciurus hudsonicus $(5,13,16)$

T. h. gymnicus $(4,9,13)$

Tamiasciurus spp. (16)

Tamias striatus $(10,11,14,16)$

T. s. griseus (16)

Zapus spp. (10)

Mouse (9)

Dist.: Alta, Man, NB, NS, Ont, PEI, Que, Sask Records: 1. Rothschild 1904 (Alta); 2. Jameson 1943 (Ont); 3. Brown 1944 (Alta); 4. Baker 1946 (Que); 5. Holland 1949 (Alta, Man, Ont, Sask); 6. Hubbard 1949a (Ont); 7. Jameson 1950 (Ont); 8. Judd 1950 (Ont); 9. Brown 1955 (NB, NS, Ont); 10. Robert 1962 (Que); 11. Buckner 1964 (Man); 12. Hopkins and Rothschild 1966 (Alta, Man, Ont); 13. Brown 1968 (NB); 14. Wright 1979 (NS); 15. Whitaker and French 1982 (NB); 16. Holland 1984 (Alta, Man, NB, NS, Ont, PEI, Que, Sask)

Delotelis hollandi Smit, 1952

Hosts: Clethrionomys gapperi $(1,2,3)$ Clethrionomys spp. (3)

Microtus longicaudus $(1,2,3)$

$M$. montanus canescens $(2,3)$

$M$. oregoni serpens $(1,2,3)$

M. townsendii (3)

M. t. townsendii $(1,2)$

Peromyscus maniculatus $(1,2)$

P. m. austerus $(1,2,3)$

P. m. oreas $(2,3)$

Peromyscus spp. (3)

Dist.: Alta, BC

Records: 1. Holland 1949 (BC); 2. Smit 1952

(BC); 3. Holland 1984 (Alta, BC)

Delotelis telegoni (Rothschild, 1905)

Syn.: Ceratophyllus telegoni Rothschild, 1905

Hosts: Clethrionomys gapperi (4)

C. g. saturatus $(1,2)$ 
Microtus longicaudus $(3,4)$

M. pennsylvanicus drummondii $(1,2)$ Microtus spp. (4)

Dist.: Alta, BC

Records: 1. Rothschild 1905 (Alta, BC); 2.

Brown 1944 (Alta); 3. Holland 1949 (BC), 4. 1984 (Alta, BC)

\section{Doratopsylla blarinae Fox, 1914}

Hosts: Blarina brevicauda $(2,3,4,5,7,8,9)$

B. b. manitobensis (6)

B. b. talpoides $(1,9)$

Blarina spp. (9)

Parascalops breweri (2)

Peromyscus spp. $(2,9)$

Sorex spp. (2)

Dist.: BC, Man, NB, NS, Ont, Que

Records: 1. Jameson 1943 (Ont), 2. Holland

1949 (Ont); 3. Jameson 1950 (Ont); 4. Robert

1962 (Que); 5. Hopkins and Rothschild 1966

(BC); 6. Buckner and Blasko 1969 (Man); 7.

Wright 1979 (NS); 8. Whitaker and French

1982 (NB); 9. Holland 1984 (NB, NS, Ont,

Que)

Epitedia faceta (Rothschild, 1915)

Syn.: Neopsylla faceta Rothschild, 1915

Hosts: Glaucomys sabrinus

Dist.: Ont, Que
$G$. volans
G. v. volans
Glaucomys spp.

Record: Holland 1984

Epitedia scapani (Wagner, 1936)

Syn.: Neopsylla scapani Wagner, 1936

Hosts: Aplodontia r. rufa (5)

Microtus oregoni serpens $(5,6,7)$

M. t. townsendii (7)

Mustela spp. (3)

Neurotrichus gibbsii (7)

N. g. gibbsii $(4,7)$

Neurotrichus spp. (7)

Peromyscus maniculatus (7)

$P$. m. austerus $(3,4,7)$

P. m. oreas (4)

Peromyscus spp. $(5,7)$

Rattus norvegicus (5)

Scapanus orarius (1)

S. o. orarius (7)

S. o. schefferi $(2,3,4,7)$

S. townsendii (3)

Sorex t. trowbridgii (5)

Sorex spp. $(3,4,7)$

Zapus spp. (7)

Mole (7)

Weasel (7)

Dist.: BC

Records: 1. Wagner 1936, 2. 1940; 3. Holland

1941, 4. 1942, 5. 1949; 6. Hopkins and

Rothschild 1962; 7. Holland 1984

Epitedia stewarti Hubbard, 1940

Host: Peromyscus spp.
Dist.: BC

Record: Holland 1984

Epitedia wenmanni wenmanni (Rothschild, 1904)

Syn.: Ctenophthalmus wenmanni Rothschild, 1904

Hosts: Blarina brevicauda $(7,8,20)$

Blarina spp. (20)

Clethrionomys gapperi $(5,13,16,20)$

C. g. athabascae (20)

C. g. loringi (20)

C. g. ochraceus (8)

C. g. proteus $(12,20)$

C. g. ungava $(11,20)$

C. rutilus (20)

C. r. dawsoni $(10,20)$

Condylura cristata $(4,18,20)$

Eutamias spp. (5)

Glaucomys sabrinus (16)

G. s. macrotis (16)

Martes americana (20)

Microtus chrotorrhinus $(19,20)$

$M$. longicaudus (20)

M. montanus canescens (20)

$M$. ochrogaster minor $(12,20)$

$M$. pennsylvanicus (18)

M. p. drummondii $(5,20)$

M. p. enixus (11)

M. p. pennsylvanicus $(4,5)$

Microtus spp. $(5,20)$

Mus musculus (5)

Mustela erminea $(5,12,20)$

M. e. cicognani $(12,20)$

M. e. invicta (20)

M. e. richardsonii (20)

$M$. frenata $(12,20)$

M. f. longicauda (12)

Mustela spp. $(12,20)$

Mustelidae gen. spp. (20)

Neotoma cinerea $(1,20)$

Neotoma spp. (20)

Onychomys leucogaster $(12,20)$

Peromyscus leucopus $(3,18)$

$P$. maniculatus $(1,5,13,14,20)$

P. m. abietorum $(9,16)$

$P$. $m$. algidus $(20)$

P. m. artemisiae $(5,12,20)$

$P$. m. bairdii $(15,20)$

P. $m$. borealis $(5,12,20)$

$P$. m. gracilis $(4,5)$

P. m. nebrascensis (5)

Peromyscus spp. (20)

Phenacomys intermedius celatus $(11,20)$

Phenacomys spp. (20)

Sorex cinereus (20)

S. c. cinereus (17)

S. fumeus (19)

Spermophilus richardsonii (20)

S. r. richardsonii (5)

Sylvilagus floridanus (20)

Synaptomys borealis (19)

S. cooperi (19) 
Tamiasciurus hudsonicus $(5,20)$

T. h. gymnicus $(9,16)$

T. h. streatori (5)

Tamiasciurus spp. (20)

Tamias striatus lysteri (2)

Vulpes vulpes (8)

Zapus princeps minor (5)

Dist.: Alta, BC, Man, NB, Nfld, NS, NWT, Ont, Que, Sask, YT

Records: 1. Rothschild 1904 (BC); 2. Jameson 1943 (Ont); 3. Brown 1944 (Alta); 4. Baker 1946 (Que); 5. Holland 1949 (Alta, BC, Man, NB, NWT, Ont, Sask); 6. Hubbard 1949a (Ont); 7. Jameson 1950 (Ont); 8. Judd 1953 (Ont); 9. Brown 1955 (NB); 10. Judd 1955 (NWT); 11. Gabbutt 1961 (Nfld); 12. Hopkins and Rothschild 1962 (Alta, BC, Man, NB, Que); 13. Robert 1962 (Que); 14. Scholten et al. 1962 (Ont); 15. Buckner 1964 (Man); 16. Brown 1968 (NB); 17. Buckner and Blasko 1969 (Man); 18. Wright 1979 (NS); 19. Whitaker and French 1982 (NB); 20. Holland 1984 (Alta, BC, Man, NB, Nfld, NS, NWT, Ont, Que, Sask, YT) Remarks: Holland (1949) recorded Putorius longicaudatus and Putorius spp. as the hosts. We recorded it under Mustelidae gen. spp. because the validity of the host classification was uncertain.

Hystrichopsylla dippiei ssp. Wilson and Johnson, 1971

Hosts: Martes a. americana (2)

Tamiasciurus hudsonicus (3)

Ground squirrels (1)

Dist.: Alta, NB, Ont

Records: 1. Gibbons and Humphreys 1941

(Alta); 2. Holland 1979 (Ont), 3. 1984 (NB, Ont)

Hystrichopsylla dippiei dippiei Rothschild, 1902

Hosts: Clethrionomys gapperi (8)

C. g. loringi $(7,8)$

Eutamias amoenus affinis (2)

Lepus spp. (3)

M. ochrogaster minor $(6,8)$

M. pennsylvanicus $(5,8)$

Microtus spp. (8)

Mustela frenata longicauda $(1,6)$

$M$. vison energumenos (1)

Peromyscus maniculatus $(5,8)$

P. m. bairdii $(7,8)$

P. m. nebrascensis (4)

Peromyscus sp. (2)

Sorex spp. (2)

Spermophilus franklinii (6)

S. richardsonii $(5,6)$

S. r. richardsonii (4)

Spilogale putorius gracilis (2)

Thomomys talpoides (3)

Zapus princeps (5)

Z. p. minor (4)

Dist.: Alta, BC, Man, Ont, Sask

Records: 1. Rothschild 1902 (Alta, BC); 2.

Wagner 1936a (BC); 3. Brown 1944 (Alta); 4.

Holland 1949 (Alta, Sask), 5. 1957 (Alta, Sask);
6. Hopkins and Rothschild 1962 (Alta, Man, Ont); 7. Buckner 1964 (Man); 8. Holland 1984 (Alta, BC, Man)

Remarks: Wagner (1936a) reported the mouse host as Peromyscus eusterugus. We could not determine the validity of this species, and so recorded it as Peromyscus sp.

Hystrichopsylla dippiei spinata Holland, 1947

Syn.: Hystrichopsylla spinata Holland, 1949

Hosts: Peromyscus maniculatus $(1,3)$ Spermophilus columbianus $(1,3)$

Dist.: BC

Spilogale putorius gracilis $(1,2)$

Records: 1. Holland 1947, 2. 1949; 3. Hopkins and Rothschild 1962

Remarks: Holland $(1947,1949)$ used Spilogale gracilis olympica instead of $S$. putorius gracilis.

Hystrichopsylla dippiei truncata Holland, 1957

Hosts: Clethrionomys gapperi (1)

C. g. saturatus (3)

Microtus longicaudus (1)

$M$. montanus canescens $(1,3)$

M. pennsylvanicus (1)

M. p. drummondii (3)

Mustela erminea (1)

Peromyscus maniculatus (1)

$P$. m. artemisiae (3)

Peromyscus spp. (3)

Sorex spp. $(1,3)$

Spermophilus columbianus $(1,2,3)$

Dist.: BC

Tamiasciurus hudsonicus (1)

Records: 1. Holland 1957; 2. Hopkins and Rothschild 1962; 3. Holland 1984

Hystrichopsylla occidentalis occidentalis Holland, 1949

Syn.: Hystrichopsylla gigas dippiei Rothschild, 1902

Hosts: Clethrionomys gapperi $(2,3)$

C. g. caurinus (1)

C. rutilus dawsoni (2)

Clethrionomys spp. (3)

Microtus longicaudus (2)

M. l. macrurus (3)

M. oeconomus (2)

$M$. oregoni serpens $(2,3,4)$

M. townsendii (2)

Microtus spp. (2)

Neurotrichus gibbsii (2)

Peromyscus maniculatus (2)

P. m. alpinus (3)

P. m. austerus (4)

Peromyscus spp. $(3,4)$

Phenacomys intermedius (2)

Rattus norvegicus (2)

R. rattus (2)

Scapanus orarius schefferi (2)

Scapanus spp. (4)

Sorex vagrans obscurus (2)

S. trowbridgii (2) 
Sorex spp. (2)

Tamiasciurus douglasii (2)

Zapus trinotatus trinotatus (4)

Dist.: Alta, BC

Records: 1. Holland 1949 (BC), 2. 1957 (BC); 3.

Campos and Stark 1979 (Alta, BC); 4. Holland

1984 (BC)

Hystrichopsylla schefferi Chapin, 1919

Hosts: Mustela vison energumenos

Dist.: BC Spilogale putorius gracilis

Record: Holland 1949

Hystrichopsylla tahavuana Jordan, 1929

Hosts: Blarina brevicauda $(3,4)$

B. b. talpoides (1)

Microtus pinetorum scalapsoides (2)

Microtus spp. (5)

Parascalops breweri (2)

Dist.: NB, Ont, Que

Records: 1. Jameson 1943 (Ont); 2. Holland 1949 (Ont); 3. Jameson 1950 (Ont); 4. Whitaker and French 1982 (NB); 5. Holland 1984 (Que)

Megarthroglossus divisus divisus (Baker, 1895)

Syn.: Megarthroglossus longispinus (Baker, 1895)

Pulex longispinus Baker, 1895

Hosts: Microtus longicaudus (3)

Mus spp. (1)

Neotoma cinerea (5)

N. c. drummondii $(3,4,5)$

Neotoma spp. (5)

Tamiasciurus hudsonicus (5)

T. h. richardsoni (1)

Dist.: Alta, BC

Records: 1. Jordan and Rothschild 1915 (Alta);

2. Wagner 1936a (BC); 3. Holland 1949 (BC); 4.

Hopkins and Rothschild 1962 (Alta); 5.

Holland 1984 (Alta)

Megarthroglossus divisus exsecatus Wagner, 1936

Hosts: Glaucomys sabrinus $(4,6)$

G. s. fuliginosus (3)

Mustela spp. $(5,6)$

Neotoma cinerea $(4,6)$

Peromyscus maniculatus (3)

Sciurus spp. (6)

Tamiasciurus hudsonicus $(3,5)$

T. h. columbiensis (3)

T. h. streatori $(1,2,3)$

Tamiasciurus spp. $(4,6)$

Dist.: BC

Weasel (3)

Records: 1. Wagner 1936; 2. Holland 1941a, 3.

1949; 4. Mendez 1956; 5. Hopkins and

Rothschild 1962; 6. Holland 1984

Megarthroglossus procus procus Jordan and

Rothschild, 1915

Syn.: Megarthroglossus similis Wagner, 1936

Hosts: Glaucomys spp. (6)
Neotoma cinerea occidentalis (2)

Peromyscus maniculatus (5)

Peromyscus spp. $(2,6)$

Rattus norvegicus (3)

Spilogale putorius gracilis (1)

Spilogale spp. (2)

Tamiasciurus douglasii $(4,6)$

T. d. mollipilosus (3)

Squirrel (6)

Dist.: $\mathrm{BC}$

Records: 1. Jordan and Rothschild 1915; 2. Wagner 1936a; 3. Holland 1949; 4. Mendez 1956; 5. Hopkins and Rothschild 1962; 6.

Holland 1984

Megarthroglossus sicamus Jordan and Rothschild, 1915

Hosts: Canis latrans (1)

Lynx spp. (3)

Neotoma cinerea occidentalis (2)

Neotoma spp. (4)

Dist.: BC

Records: 1. Jordan and Rothschild 1915; 2.

Wagner 1936a; 3. Holland 1949, 4. 1984

Megarthroglossus spenceri Wagner, 1936

Syn.: Megarthroglossus pygmaeus Wagner, 1936

Hosts: Neotoma cinerea $(1,2)$

Dist.: BC

$$
\text { Ochotona princeps (1) }
$$

Records: 1. Wagner 1936a; 2. Tipton et al. 1979

Meringis parkeri Jordan, 1937

Host: Dipodomys ordii terrosus

Dist.: Sask

Record: Holland 1984

Meringis shannoni (Jordan, 1929)

Hosts: Perognathus parvus laingi (2)

P. p. lordi $(1,2)$

Peromyscus maniculatus artemisiae (1)

Dist.: BC

Records: 1. Holland 1941a, 2. 1949

Nearctopsylla brooksi (Rothschild, 1904)

Hosts: Canis spp. $(3,4)$

Lynx spp. (3)

Martes americana $(1,2,3,4)$

Martes a. abietinoides (4)

Mustela erminea $(2,4)$

M. e. cicognanii (4)

M. e. invicta $(1,3,4)$

M. e. richardsonii $(1,2,3,4)$

M. frenata $(2,4)$

M. f. longicauda (1)

M. f. nevadensis (4)

$M$. f. oribasus $(2,4)$

Mustela vison $(2,3,4)$

$M$. v. energumenos (4)

Mustela spp. $(2,3,4)$

Spermophilus richardsonii (3)

Marten (4) 
Weasel (4)

Dist.: Alta, BC, YT

Records: 1. Rothschild 1904 (Alta, BC); 2.

Holland 1949 (Alta, BC); 3. Hopkins and

Rothschild 1962 (Alta, BC); 4. Holland 1984

(Alta, BC, YT)

Remarks: Holland (1984) reported three species: Putorius cicognani (= Mustela erminea cicognanii), Putorius richardsonii $(=M . e$.

richardsonii) and Mustela erminea richardsonii.

Nearctopsylla genalis (Baker, 1904)

Host: Blarina brevicauda (1)

Dist.: Ont

Record: Jameson (1950)

Nearctopsylla genalis genalis (Baker, 1904)

Syn.: Nearctopsylla hygini laurentina Jordan and Rothschild, 1923

Hosts: Blarina brevicauda $(5,8)$

Blarina spp. (8)

Clethrionomys gapperi $(6)$

Condylura cristata $(7,8)$

Martes americana $(8)$

Mustela erminea $(2,8)$

M. e. cicognanii $(3,8)$

M. e. richardsonii (6)

M. frenata noveboracensis $(5,8)$

Mustela spp. $(1,2)$

Scalopus spp. (8)

Sorex arcticus (4)

S. cinereus $(8)$

S. c. $\operatorname{miscix}(5,8)$

S. fumeus (8)

Tamiasciurus hudsonicus gymnicus (6)

Weasel (8)

Dist.: NB, Nfld, Ont, Que

Records: 1. Jordan and Rothschild 1923 (NB);

2. Holland 1949 (NB, Ont); 3. Judd 1954a

(Ont); 3. Brown 1955 (NB); 4. Hopkins and

Rothschild 1962 (Nfld, Que); 5. Brown 1968

(NB); 6. Whitaker and French 1982 (NB); 7.

Holland 1984 (NB, Nfld, Ont, Que)

Nearctopsylla genalis hygini (Rothschild, 1904)

Syn.: Ctenopsyllus hygini Rothschild, 1904

Hosts: Blarina brevicauda (5)

B. b. manitobensis (5)

Mustela erminea $(2,5)$

M. e. $\operatorname{arctica}(5)$

M. e. bangsi (3)

M. e. cicognanii (5)

M. e. invicta (1)

M. e. richardsonii (5)

Mustela sp. $(3,5)$

Sorex $\operatorname{arcticus}(5)$

S. a. $\operatorname{arcticus}(5)$

Sorex spp. $(2,5)$

Dist.: Alta, Man, Sask

Records: 1. Rothschild 1904 (Alta); 2. Holland 1949 (Alta, Man, Sask); 3. Hopkins and

Rothschild 1962 (Alta, Man); 4. Buckner and

Blasko 1969 (Man); 5. Holland 1984 (Alta,

Man, Sask)
Remarks: Holland (1984) classified Mustela e.

richardsonii and M. e. arctica as Putorius

richardsonii and $M$. arctica arctica respectively.

Nearctopsylla grahami Holland, 1979

Host: Martes a. americana (1)

Dist.: Ont

Records: 1. Holland 1979, 2. 1984

Nearctopsylla hyrtaci (Rothschild, 1904)

Syn.: Ctenopsyllus hyrtaci Rothschild, 1904

Hosts: Clethrionomys gapperi saturatus (2)

Martes americana $(2)$

M. a. caurina (2)

Microtus spp. (4)

Mustela erminea (3)

M. e. cicognanii (4)

$M$. frenata $(2,4)$

M. f. longicauda (3)

M. vison (2)

$M$. v. energumenos $(1,4)$

Mustela spp. $(2,3,4)$

Sorex $a$. arcticus $(3,4)$

S. cinereus (2)

$S$. vagrans $(1,4)$

S. v. setosus (4)

S. palustris (4)

Sorex spp. $(3,4)$

Weasel (4)

Dist.: Alta, BC

Records: 1. Rothschild 1904 (BC); 2. Holland 1949 (BC); 3. Hopkins and Rothschild 1962

(Alta, BC); 4. Holland 1984 (Alta, BC)

Nearctopsylla jordani Hubbard, 1940

Syn.: Nearctopsylla hygini columbiana Wagner, 1940

Hosts: Neurotrichus gibbsii $(4,5)$

N. g. gibbsii $(3,5)$

Neurotrichus spp. (5)

Scapanus orarius schefferi $(1,2,5)$

Dist.: BC

Scapanus spp. (5)

Records: 1. Wagner 1940; 2. Holland 1941a, 3. 1949; 4. Hopkins and Rothschild 1962; 5.

Holland 1984

Nearctopsylla traubi Hubbard, 1940

Hosts: Microtus oregoni serpens $(2,4)$ Neurotrichus g. gibbsii $(1,2,4)$

Scapanus orarius schefferi $(2,3,4)$

S. townsendii (2)

Dist.: BC

Records: 1. Holland 1949; 2. Smit 1952; 3.

Hopkins and Rothschild 1962; 4. Holland 1984

Neopsylla inopina Rothschild, 1915

Syn.: Epitedia inopina (Rothschild, 1915)

Hosts: Clethrionomys gapperi $(1,8)$

C. g. saturatus (4)

Mustela frenata longicauda $(1,4,6)$

Mustela spp. (6)

Peromyscus maniculatus nebrascensis (6) 
Rattus norvegicus ( 8 )

Spermophilus columbianus $(6,7)$

S. c. columbianus $(2,5,8)$

S. franklinii (6)

S. richardsonii $(1,4,5,7,8)$

S. r. richardsonii $(6,7)$

Ground squirrels (3)

Weasel (8)

Dist.: Alta, BC, Sask

Records: 1. Rothschild 1915 (Alta); 2. Holland 1940 (BC); 3. Gibbons and Humphreys 1941 (Alta); 4. Brown 1944 (Alta); 5. Holland 1944 (Alta, BC, Sask), 6. 1949 (Alta, BC, Sask); 7. Hilton and Mahrt 1971 (Alta); 8. Holland 1984 (Alta, BC, Sask)

Rhadinopsylla arborea Smit, 1957

Hosts: Clethrionomys gapperi (2)

Clethrionomys spp. (2)

Martes americana (2)

Microtus longicaudus (2)

Neotoma cinerea (2)

N. c. drummondii (2)

Tamiasciurus hudsonicus (2)

T. h. baileyi $(1,2)$

Dist.: Alta, BC

Record: 1. Smit 1957 (Alta); 2. Holland 1984

(Alta, BC)

Rhadinopsylla difficilis Smit, 1957

Hosts: Microtus montanus canescens (3)

Mustela erminea $(1,2,3)$

Mustela spp. $(1,2,3)$

Neotoma spp. (3)

Ochotona princeps (3)

O. p. brooksi (3)

Peromyscus spp. (3)

Phenacomys spp. (3)

Dist.: BC

Spermophilus lateralis (3)

Records: 1. Smit 1957; 2. Hopkins and

Rothschild 1962; 3. Holland 1984

Rhadinopsylla fraterna (Baker, 1895)

Syn.: Rectofrontia fraterna (Baker, 1895)

Hosts: Clethrionomys gapperi (8)

Lagurus curtatus (8)

Martes americana (3)

M. a. americana (7)

Microtus pennsylvanicus (8)

M. p. drummondii $(5,8)$

Mustela frenata longicauda $(3,4,5,8)$

Mustela spp. $(5,8)$

Neotoma cinerea drummondii (3)

Ochotona princeps $(3,4)$

Onychomys leucogaster missouriensis

$(3,8)$

Peromyscus maniculatus (8)

P. m. nebrascensis (3)

Phenacomys intermedius (3)

Spermophilus c. columbianus (1)

S. richardsonii $(4,5,6,8)$

S. r. richardsonii (3)
Tamiasciurus hudsonicus (3)

Thomomys spp. $(5,8)$

Ground squirrels (2)

Dist.: Alta, BC, Ont, Sask

Records: 1. Holland 1940c (BC); 2. Gibbons and Humphreys 1941 (Alta); 3. Holland 1949

(Alta, BC, Sask); 4. Smit 1957 (Alta); 5.

Hopkins and Rothschild 1962 (Alta); 6. Hilton and Mahrt 1971 (Alta); 7. Holland 1979 (Ont), 8. 1984 (BC, Sask)

Rhadinopsylla linta Smit, 1957

Hosts: Clethrionomys gapperi athabascae (2)

C. rutilus dawsoni $(1,2)$

Clethrionomys spp. (2)

Lemmus sibiricus (2)

Microtus spp. (2)

Neotoma cinerea occidentalis $(1,2)$

Peromyscus maniculatus (2)

Spermophilus parryii (2)

Spermophilus spp. (2)

Tamiasciurus hudsonicus (2)

Dist.: BC, NWT, YT

Records: 1. Smit 1957 (BC); 2. Holland 1984

(BC, NWT, YT)

Rhadinopsylla media Smit, 1957

Hosts: Clethrionomys gapperi (2)

C. g. proteus $(1,2)$

Mustela erminea (2)

Mustela spp. (2)

Dist.: Alta, Que

Records: 1. Smit 1957 (Que); 2. Hopkins and Rothschild 1962 (Alta, Que)

Rhadinopsylla rauschi Holland, 1979

Host: Peromyscus maniculatus nebrascensis

Dist.: Sask

Records: 1. Holland 1979, 2. 1984

Rhadinopsylla sectilis goodi (Hubbard, 1941)

Syn.: Rectofrontia sectilis Wagner, 1936

Hosts: Microtus oregoni serpens (2)

Peromyscus maniculatus (3)

$P$. m. austerus $(1,2,3)$

P. $m$. oreas $(2,3)$

Peromyscus spp. $(2,3)$

Rattus norvegicus $(2,3)$

Spilogale putorius gracilis $(2,3)$

Dist.: BC

Tamiasciurus douglasii mollipilosus (2)

Records: 1. Wagner 1936a; 2. Holland 1949, 3. 1984

Rhadinopsylla sectilis sectilis Jordan and Rothschild, 1923

Hosts: Mus musculus (2)

Mus spp. (1)

Peromyscus maniculatus (2)

P. m. artemisiae $(1,2,3)$

Peromyscus spp. (3)

Spermophilus c. columbianus (2)

Tamiasciurus hudsonicus (3) 
T. h. streatori (2)

Dist.: BC

Records: 1. Jordan and Rothschild 1923; 2.

Holland 1949, 3. 1984

Stenistomera alpina (Baker, 1895)

Hosts: Mus spp. (1)

Neotoma cinerea (1)

Peromyscus maniculatus (2)

Dist.: Alta

Records: 1. Holland 1952a; 2. Hopkins and

Rothschild 1962

Stenoponia americana (Baker, 1899)

Syn.: Hystrichopsylla americana Baker, 1899

Hosts: Blarina brevicauda (8)

B. b. manitobensis (6)

B. b. talpoides $(3,8)$

Clethrionomys gapperi $(4,5,8)$

C. g. loringi $(6,8)$

C. g. ochraceus $(1,3)$

Clethrionomys spp. (8)

Microsorex h. hoyi $(6,8)$

Microtus ochrogaster minor (4)

Mus spp. (4)

Napaeozapus insignis (7)

N. i. insignis (8)

Peromyscus maniculatus abietorum (3)

P. m. gracilis $(2,3)$

Tamiasciurus hudsonicus (5)

Dist.: Alta, Man, NB, NS, Ont, Que

Records: 1. Morris 1943 (NB, Que); 2. Holland 1949 (Man, Ont); 3. Brown 1955 (NB, Que); 4.

Hopkins and Rothschild 1962 (Alta, Man,

Que); 5. Brown 1968 (NB); 6. Buckner and

Blasko 1969 (Man); 7. Wright 1979 (NS); 8.

Holland 1984 (Man, NS, Ont, Que)

Tamiophila grandis (Rothschild, 1902)

Syn.: Typhlopsylla grandis Rothschild, 1902

Hosts: Sylvilagus floridanus mearnsi (3)

Tamias striatus $(1,2,4,7,8,9)$

T. s. griseus (4)

T. s. lysteri $(3,5,6)$

Chipmunk (4)

Cottontail rabbit (4)

Dist.: Ont, Que

Records: 1. Rothschild 1902 (Ont); 2. Wagner

1936 (Que); 3. Jameson 1943 (Ont); 4. Holland

1949 (Ont); 5. Judd 1950 (Ont), 6. 1954a (Ont);

7. Scholten et al. 1962 (Ont); 8. Brown 1968

(Ont); 9. Holland 1984 (Ont)

Trichopsylloides oregonensis Ewing, 1938

Host: Mustela vison energumenos

Dist.: BC

Record: Holland 1941a

Family ISCHNOPSYLLIDAE

Myodopsylla gentilis Jordan and Rothschild, 1921

Hosts: Myotis lucifugus $(3,5)$

M. l. alascensis $(3,5)$

M. l. lucifugus (5)
M. yumanensis saturatus (3)

Myotis spp. (5)

Bat $(1,2,3,4,5)$

Dist.: Alta, BC, Ont, Que

Records: 1. Jordan and Rothschild 1921 (BC);

2. Holland 1939b (BC), 3. 1949 (BC); 4.

Hopkins and Rothschild 1956 (Alta); 5.

Holland 1984 (Alta, BC, Ont, Que)

Myodopsylla insignis (Rothschild, 1903)

Syn.: Ceratopsylla insignis Rothschild, 1903

Hosts: Eptiscus fuscus (7)

E.f. fuscus (16)

E. f. pallidus (5)

Myotis keeni septentrionalis (5)

M. lucifugus $(1,3,5,6,7,11,15,16)$

M. l. lucifugus $(2,4,9,13)$

M. l. pernox (16)

Myotis spp. $(5,12,14,16)$

Bat $(7,8,10,16)$

Brown bat (5)

Dist.: Alta, BC, Man, NB, Nfld, NS, Ont, PEI,

Que, Sask, YT Records: 1. Rothschild 1903

(Ont); 2. Dymond 1938 (Ont); 3. Fuller 1943

(Ont); 4. Jameson 1943 (Ont); 5. Holland 1949

(Alta, BC, Ont, Sask); 6. Judd 1953 (Ont); 7.

Hopkins and Rothschild 1956 (Ont, Que); 8.

Smit 1958 (Alta); 9. Harper 1961 (Nfld); 10.

Robert 1962 (Que); 11. Wilson 1967 (NB); 12.

Brown 1968 (NB); 13. Buckner 1971 (Man); 14.

Wright 1979 (NS); 15. Jones and Thomas 1983

(PEI); 16. Holland 1984 (Alta, NS, Ont, Que, YT)

Myodopsylla palposa (Rothschild, 1904)

Syn.: Ceratopsylla palposus Rothschild, 1904 Myodopsylloides palposus (Rothschild, 1904)

Rhinolophopsylla palposus (Rothschild, 1904)

Hosts: Eptesicus fuscus $(6,7,8)$

E. f. fuscus $(2,3,4,5)$

Mus musculus (8)

Bat (8)

Dist.: BC

Brown bat $(1,6)$

Records: 1. Rothschild 1904; 2. Wagner 1936a; 3. Spencer 1939a; 4. Wagner 1940; 5. Holland 1941a, 6. 1949; 7. Hopkins and Rothschild 1956; 8. Holland 1984

Nycteridopsylla vancouverensis Wagner, 1936

Host: Lasionycteris noctivagans

Dist.: BC

Records: Wagner 1936; Holland 1984

Family LEPTOPSYLLIDAE

Amphipsylla sibirica pollionis (Rothschild, 1905)

Syn.: Ceratophyllus pollionis Rothschild, 1905

Hosts: Clethrionomys gapperi (5)

C. g. athabascae (4)

C. g. loringi (1)

C. g. saturata $(2,3)$ 
C. rutilus (5)

C. r. dawsoni (5)

Clethrionomys spp. (5)

Lemmus sibiricus (5)

Microtus longicaudus (5)

M. oeconomus (5)

M. pennsylvanicus (5)

M. p. drummondii $(1,2,3,5)$

M. xanthognathus (5)

Microtus spp. (5)

Peromyscus maniculatus (5)

Synaptomys spp. (5)

Dist.: Alta, BC, Nfld, YT

Records: 1. Rothschild 1905 (Alta); 2. Wagner 1936a (Alta); 3. Brown 1944 (Alta); 4. Holland 1949 (Alta), 5. 1984 (Alta, BC, Nfld, YT)

Amphipsylla washingtona Hubbard, 1954

Host: Pallid vole

Dist.: Alta

Record: Holland 1984

Ctenophyllus armatus (Wagner, 1901)

Syn.: Ctenophyllus terribilis Rothschild, 1903

Hosts: Ochotona collaris (7)

$$
\begin{aligned}
& \text { O. princeps }(1,2,3,5,7) \\
& \text { O. p. brooksi }(4,5,6,7) \\
& \text { O. p. cuppes }(4,5) \\
& \text { O. p. fenisex }(5) \\
& \text { O. p. princeps }(5) \\
& \text { Ochotona spp. (7) }
\end{aligned}
$$

Dist.: Alta, BC, YT

Records: 1. Rothschild 1903 (Alta); 2. Wagner 1936a (Alta); 3. Holland 1940c (BC); 4. Jellison 1941 (BC); 5. Holland 1949 (Alta, BC); 6.

Hopkins and Rothschild 1971 (BC); 7. Holland 1984 (Alta, BC, YT)

Dolichopsyllus stylosus (Baker, 1904)

Hosts: Aplodontia rufa (3)

$$
\text { A. r. rufa (1) }
$$

Mustela vison energumenos $(2,3)$

Dist.: BC

Records: 1. Holland 1949; 2. Hopkins and

Rothschild 1971; 3. Holland 1984

Leptopsylla segnis (Schnherr, 1811)

Syn.: Ctenopsyllus segnis (Schonherr, 1811)

Host: Mus musculus

Dist.: BC, Nfld

Records: Holland 1941a (BC), 1984 (Nfld)

Odontopsyllus dentatus (Baker, 1904)

Hosts: Sylvilagus nuttallii grangeri

Dist.: Alta, Sask

$$
\text { Sylvilagus spp. }
$$

Records: Holland 1949

Odontopsyllus multispinosus (Baker, 1898)

Host: Silvilagus spp.

Dist.: Alta

Record: Brown 1944
Peromyscopsylla catatina (Jordan, 1928)

Hosts: Blarina brevicauda angusta (7)

B. b. manitobensis ( 8 )

Clethrionomys gapperi $(1,5,7,10,12)$

C. g. gaspeanus (7)

C. g. loringi $(6,12)$

C. g. ochraceus (3)

C. g. proteus $(2,12)$

Clethrionomys spp. (12)

Microtus chrotorrhinus $(11,12)$

M. c. chrotorrhinus $(1,7)$

M. pennsylvanicus (12)

M. p. drummondii (6)

Napaeozapus insignis (12)

N. i. frutectanus (1)

N. i. insignis (12)

Peromyscus maniculatus $(4,5)$

P. m. bairdii $(8,12)$

Sorex cinereus (12)

S. c. cinereus $(8)$

Synaptomys spp. (7)

Tamiasciurus hudsonicus (12)

T. h. hudsonicus (1)

Dist.: Man, NB, Nfld, NS, NWT, Ont, Que Records: 1. Holland 1949 (Ont); 2. Johnson and Traub 1954 (Que, Nfld); 3. Brown 1955 (NB); 4. Harper 1956 (NWT); 5. Robert 1962 (Que); 6. Buckner 1964 (Man); 7. Brown 1968 (NB); 8. Buckner and Blasko 1969 (Man); 9.

Hopkins and Rothschild 1971 (Nfld); 10. Wright 1979 (NS); 11. Whitaker and French 1982 (NB); 12. Holland 1984 (Man, Nfld, NS, Ont)

Remarks: Harper (1956) recorded the host as Peromyscus maniculatus drummondi. Because of the doubtful nature of this subspecies we used $P$. maniculatus.

Peromyscopsylla hamifer hamifer (Rothschild, 1906) Syn.: Ctenopsyllus hamifer Rothschild, 1906

Peromyscopsylla hamifer markworthi Hubbard, 1949

Hosts: Clethrionomys gapperi (8)

C. g. loringi $(6,8)$

C.g.proteus (5)

C. rutilus (8)

Microtus longicaudus $(7,8)$

M. pennsylvanicus $(4,5,7,8)$

M. p. drummondii (8)

M. p. enixus $(7,8)$

M. p. labradorius $(5,7,8)$

M. p. pennsylvanicus (3)

M. xanthognathus (8)

Microtus spp. (8)

Mustela erminea $(5,6)$

M. e. cicognani (5)

Mustela spp. $(1,2,5,7,8)$

Mus spp. (7)

Sorex cinereus (8)

Dist.: Alta, BC, Man, Nfld, NWT, Ont, Que, Sask

Records: 1. Rothschild 1906 (Alta); 2. Brown 
1944 (Alta); 3. Baker 1946 (Que); 4. Hubbard 1949a (Ont); 5. Johnson and Traub 1954 (Alta, Nfld, NWT, Ont, Que); 6. Buckner and Blasko 1969 (Man); 7. Hopkins and Rothschild 1971 (Alta, BC, Nfld, Ont, Que); 8. Holland 1984 (Alta, BC, Man, Nfld, NWT, Ont, Sask)

Peromyscopsylla hesperomys adelpha (Rothschild, 1915)

Hosts: Clethrionomys spp. (2)

Lagurus curtatus (2)

Peromyscus spp. $(1,2)$

Dist.: Alta, Sask

Records: 1. Johnson and Traub 1954 (Alta); 2. Holland 1984 (Alta, Sask)

Peromyscopsylla hesperomys hesperomys (Baker, 1904)

Hosts: Microtus spp. (4)

Peromyscus leucopus $(3,4)$

P. maniculatus (3)

Peromyscus spp. $(1,2,4)$

Dist.: NS, Ont, Que

Records: 1. Holland 1952a (Ont, Que); 2.

Johnson and Traub 1954 (Ont); 3. Wright 1979

(NS); 4. Holland 1984 (NS, Ont, Que)

Peromyscopsylla hesperomys pacifica Holland, 1949

Hosts: Microtus oregoni serpens (3)

Peromyscus maniculatus (3)

P. m. austerus $(1,2)$

P. $m$. interdictus $(1)$

P. m. oreas $(1,2)$

Dist.: BC

Peromyscus spp. (3)

Records: 1. Holland 1949; 2. Hopkins and

Rothschild 1971;3. Holland 1984

Peromyscopsylla ostsibirica longiloba (Jordan, 1939)

Hosts: Microtus oeconomus

Dist.: YT

Microtus spp.

Record: Holland 1984

Peromyscopsylla ravalliensis (Dunn, 1923)

Syn.: Ctenopsyllus ravalliensis Dunn, 1923

Ctenopsyllus rawailliensis Dunn and

Parker, 1923

Hosts: Neotoma cinerea $(2,3,4)$

N. c. occidentalis $(1,2)$

Neotoma spp. $(3,4)$

Ochotona princeps (2)

Peromyscus maniculatus (2)

Dist.: Alta, BC

Records: 1. Wagner 1936 (BC); 2. Holland 1949

(BC); 3. Hopkins and Rothschild 1971 (Alta,

BC); 4. Holland 1984 (Alta, BC)

Peromyscopsylla scotti Fox, 1939

Host: Peromyscus leucopus

Dist.: Ont

Records: 1. Holland 1952a, 2. 1984
Peromyscopsylla selenis (Rothschild, 1906)

Syn.: Ctenopsyllus selenis Rothschild, 1906

Hosts: Clethrionomys gapperi $(3,4)$

C. g. athabascae $(3,4)$

C. g. loringi $(1,4)$

C. g. saturatus $(1,3)$

C. rutilus (4)

C. r. dawsoni (4)

Clethrionomys spp. (4)

Martes americana (3)

Microtus longicaudus $(3,4)$

M. l. macrurus (4)

M. l. vellerosus (4)

$M$. oeconomus operarius (4)

$M$. oregoni serpens $(3,4)$

$M$. pennsylvanicus (4)

$M . p$. drummondii $(1,4)$

M. t. townsendii (4)

Microtus spp. $(3,4)$

Peromyscus maniculatus $(3,4)$

P. m. borealis $(1,3,4)$

Peromyscus spp. (4)

Phenacomys intermedius $(3,4)$

Rattus norvegicus (3)

Sorex spp. $(3,4)$

Spermophilus $r$. richardsonii (3)

Synaptomys b. borealis (3)

Synaptomys spp. (4)

Tamiasciurus hudsonicus (4)

Zapus hudsonius $(2,4)$

Z. princeps (4)

Zapus spp. (1)

Dist.: Alta, BC, Man, NWT, Sask, YT

Records: 1. Rothschild 1906 (Alta, BC); 2.

Brown 1944 (Alta); 3. Holland 1949 (Alta, BC,

Man, NWT, Sask), 4. 1984 (Alta, BC, Man,

NWT, Sask, YT)

\section{Family PULICIDAE}

Cediopsylla inaequalis inaequalis (Baker, 1895)

Hosts: Lepus spp. $(2,4)$

Sylvilagus nuttallii (1)

S. n. grangeri (3)

Sylvilagus spp. (3)

Vulpes vulpes (5)

Dist.: Alta, Sask

Records: 1. Holland 1941a (Alta); 2. Brown 1944 (Alta); 3. Holland 1949 (Alta, Sask); 4.

Hopkins and Rothschild 1953 (Alta); 5.

Holland 1984 (Sask)

Cediopsylla simplex (Baker, 1895)

Hosts: Lepus capensis hybridus (2)

Sylvilagus floridanus (3)

S. f. mearnsii (1)

Vulpes vulpes (3)

Cottontail rabbit (3)

Dist.: Ont

Records: 1. Holland 1949; 2. Judd 1953; 3.

Holand 1984

Ctenocephalides canis (Curtis, 1826)

Hosts: Rattus norvegicus (1) 
Spilogale putorius phenax (2)

Vulpes vulpes (2)

Dist.: BC, Ont

Records: 1. Holland 1941 (BC), 2. 1984 (BC,

Ont)

Ctenocephalides felis (Bouch, 1835)

Host: Rattus norvegicus

Dist.: BC

Record: Holland 1940c

Ctenocephalides felis felis (Bouch, 1835)

Hosts: Rattus norvegicus $(1,2)$

Dist.: BC Spilogale spp. (2)

Records: 1. Holland 1941b, 2. 1984

Euhoplopsyllus glacialis affinis (Baker, 1904)

Syn.: Hoplopsyllus affinis (Baker, 1904)

Hosts: Lepus spp.

Dist.: Sask Silvilagus floridanus

Records: Holland 1949, 1984

Euhoplopsyllus glacialis glacialis (Taschenberg, 1880)

Syn.: Hoplopsyllus glacialis (Taschenberg, 1880)

Hosts: Lepus arcticus $(1,2,4)$

L. a. $\operatorname{arcticus}(3,4)$

Dist.: NWT

Records: 1. Jordan 1932; 2. Holland 1949; 3.

Hopkins and Rothschild 1953; 4. Holland 1984

Euhoplopsyllus glacialis lynx (Baker, 1904)

Syn.: Hoplopsyllus glacialis lynx (Baker, 1904)

Hosts: Lepus americanus $(2,4,5,8)$
L. a. bairdii (4)
L. a. columbianus (4)
L. a. dalli $(1,8)$
L. a. struthopus $(6,7)$
Lepus spp. (8)
Lynx canadensis $(4,5,8)$
L. rufus (8)
L. r. baileyi $(8)$
L. r. fasciatus $(2,4,5)$
Lynx spp. $(3,5,8)$
Mustela erminea (8)
Ochotona spp. (8)
Vulpes vulpes (8)
V. v. rubricosa $(7)$
Bush rabbit $(4,8)$
Fox (8)
Hare (4)
Rabbit (8)

Dist.: Alta, BC, NB, NWT, YT

Records: 1. Jordan 1932 (BC); 2. Wagner 1936

(BC); 3. Kohls 1940 (YT); 4. Holland 1949

(Alta, BC, NB, NWT); 5. Hopkins and

Rothschild 1953 (Alta, BC, NB); 6. Brown 1955

(NB), 7. 1968 (NB); 8. Holland 1984 (Alta, BC,

NWT, YT)
Pulex irritans Linnaeus, 1758

Hosts: Canis latrans $(4,5,6)$

Lepus townsendii (6)

L. t. campanius (4)

Lepus spp. $(1,5)$

Lynx spp. $(1,5)$

Odocoileus hemionus columbianus (3)

Taxidea taxus $(2,6)$

Vulpes vulpes $(1,5,6)$

Fox (6)

Dist.: Alta, BC, Man, PEI, Sask

Records: 1. Jordan and Rothschild 1908 (Alta);

2. Brown 1944 (Alta); 3. Cowan 1946 (BC); 4.

Holland 1949 (Alta, Man, PEI); 5. Hopkins

and Rothschild 1953 (Alta, Man); 6. Holland

1984 (Man, Sask)

Xenopsylla cheopis (Rothschild, 1903)

Hosts: Rattus norvegicus $(1,2,3,4,5,6,7,8)$

$R$. rattus alexandrinus $(3,6)$

$R$. r. rattus (6)

Dist.: Alta, BC, NB, NS, Ont, Que

Records: 1. Spencer 1936 (BC); 2. Gibbons and

Humphreys 1941 (Alta); 3. Holland 1940c

(BC), 4. 1941 b (BC), 5. 1944 (BC), 6. 1949 (BC);

7. McKiel et al. 1960 (NB, NS, Ont, Que); 8.

Holland 1984 (Ont, Que)

Family VERMIPSYLLIDAE

Chaetopsylla lotoris (Stewart, 1926)

Syn.: Trichopsylla lotoris Stewart, 1926

Hosts: Procyon lotor $(1,2)$

Dist.: Ont Vulpes vulpes (2)

Records: 1. Holland 1952a, 2. 1984

Chaetopsylla setosa Rothschild, 1906

Syn.: Arctopsylla setosa (Rothschild, 1906) Trichopsylla setosus (Rothschild, 1906)

Hosts: Canis latrans (1) Erethizon dorsatum (5)

Gulo gulo luscus (4)

Lynx canadensis (4)

Ursus americanus (1)

U. $\operatorname{arctos}(2,3,4)$

Dist.: BC

Records: 1. Rothschild 1906; 2. Spencer 1936; 3. Wagner 1936; 4. Holland 1949, 5. Holland 1984

Chaetopsylla tuberculaticeps (Bezzi, 1890)

Syn.: Arctopsylla ursi (Rothschild, 1902)

Pulex ursi Rothschild, 1902

Trichopsylla ursi (Rothschild, 1902)

Hosts: Ursus americanus (5)

$U . \operatorname{arctos}(1,2,3,4,7)$

Dist.: Alta, BC

Records: 1. Rothschild 1902 (Alta); 2. Wagner 1936a (Alta); 3. Holland 1941 (BC); 4. Brown

1944 (Alta); 5. Hubbard 1947 (Alta); 6.

Hopkins and Rothschild 1956 (Alta); 7.

Holland 1984 (BC) 


\section{ORDER SIPHUNCULATA}

\section{Family HAEMATOPINIDAE}

Linognathoides montanus Osborn, 1912

Host: Spermophilus spp.

Dist.: Canada

Record: McLeod 1933

Remarks: McLeod (1933) stated that this species is found "Practically all over the North American continent".

Family HOPLOPLEURIDAE

Enderleinellus marmotae Ferris, 1919

Host: Marmota monax

Dist.: Ont

Record: Scholten et al. 1962

Enderleinellus nitschi Fahrenholz, 1916

Hosts: Napaeozapus insignis (2)

Tamiasciurus hudsonicus $(1,2)$

Dist.: Ont, Que

Records: 1. Scholten et al. 1962 (Ont); 2.

Gyorkos and Hilton 1982 (Que)

Enderleinellus suturalis (Osborn, 1891)

Hosts: Spermophilus columbianus

$$
\text { S. franklinii }
$$

S. richardsonii

Dist.: Alta

Record: Hilton and Mahrt 1971

Fahrenholzia pinnata Kellogg and Ferris, 1915

Hosts: Perognathus parvus laingi

Dist.: BC

$$
\text { P. p. lordi }
$$

Record: Spencer 1966

Haemodipsus ventricosus Penny, 1842

Hosts: Lepus capensis hybridus (1)

$$
\text { Lab rabbit (2) }
$$

Dist.: BC, Ont

Record: 1. Judd 1953 (Ont); 2. Spencer 1966

(BC)

\section{Haemodipsus spp.}

Host: Lepus europaeus

Dist.: Ont

Record: Judd 1950

Remarks: Judd (1950) believed his specimens may have been Haemodipsus ventricosus.

Hoplopleura acanthopus (Burmeister, 1839)

Hosts: Blarina brevicauda (1)

Clethrionomys gapperi (3)

C. g. saturatus (5)

Dicrostonyx groenlandicus (4)

Lemmus sibiricus trimucronatus (5)

Microtus longicaudus (5)

M. l. vellerosus (5)

M. montanus canescens (5)

$M$. oregoni serpens (5)

M. pennsylvanicus $(3,6)$

M. p. drummondii (5)
M. p. pennsylvanicus $(1,2)$

M. townsendii (5)

Neotoma cinerea occidentalis (5)

Ochotona princeps fenisex (5)

Rattus norvegicus (5)

Sorex c. cinereus (5)

Synaptomys borealis (7)

S. b. chapmani (5)

S. cooperi (7)

Thomomys talpoides fuscus (5)

Dist.: Alta, BC, NB, NWT, Ont, Sask

Records: 1. Judd 1953 (Ont), 2. 1954a (Ont); 3.

Scholten et al. 1962 (Ont); 4. McAlpine 1965

(NWT); 5. Spencer 1966 (Alta, BC, NWT,

Ont); 6. Wilson 1967 (Sask); 7. Whitaker and

French 1982 (NB)

Remarks: Spencer (1966) recorded Microtus montanus canescens as $M$. microtus canascens.

Hoplopleura arboricola (Kellogg and Ferris, 1915)

Hosts: Eutamias amoenus affinis

Dist.: $\mathbf{B C}$

E. a. luteiventris

Record: Spencer 1966

Hoplopleura erratica (Osborn, 1896)

Host: Tamias striatus

Dist.: Ont, Que

Records: Scholten et al. 1962 (Ont); Gyorkos and Hilton 1982 (Que)

Hoplopleura hesperomydis (Osborn, 1891)

Hosts: Clethrionomys gapperi (3)

Mus musculus (1)

Peromyscus maniculatus $(1,3,4,5)$

P. m. artemisiae (2)

P. m. austerus (2)

P. $m$. oreas (2)

P. m. prevostensis (2)

Peromyscus spp. (2)

Tamiasciurus hudsonicus (3)

Dist.: BC, NB, NS, Ont, Que

Records: 1. Scholten et al. 1962 (Ont); 2.

Spencer 1966 (BC); 3. Gyorkos and Hilton 1982 (Que); 4. Wright 1979 (NS); 5. Whitaker and French 1982 (NB)

Hoplopleura oenomydis Ferris, 1921

Host: Rattus norvegicus

Dist.: BC

Record: Spencer 1966

Hoplopleura sciuricola Ferris, 1921

Hosts: Tamiasciurus douglasii mollipilosus (2)

$T$. hudsonicus $(1,2,3)$

T. h. streatori (2)

Dist.: Alta, BC

Records: 1. Thompson 1934 (Alta); 2. Spencer 1966 (BC); 3. Mahrt and Chai 1972 (Alta)

Hoplopleura trispinosa Kellogg and Ferris, 1915

Hosts: Glaucomys sabrinus columbiensis (2)

G. s. oregonensis $(1,2)$ 
Dist.: Alta, BC

Records: 1. Spencer 1956 (Alta, BC), 2. 1966 (BC)

Microphthirus uncinatus (Ferris, 1916)

Syn.: Enderleinellus uncinatus Ferris, 1916

Host: Glaucomys sabrinus oregonensis

Dist.: Alta, BC

Records: Spencer 1956 (Alta, BC), 1966 (BC)

Neohaematopinus inornatus Kellogg and Ferris, 1915

Syn.: Linognathoides inornatus Kellogg and Ferris, 1916

Host: Neotoma cinerea occidentalis

Dist.: BC

Record: Spencer 1966

Neohaematopinus laeviusculus (Grube, 1851)

Hosts: Spermophilus columbianus (2)
S. c. columbianus (1)
$S$. richardsonii (2)
S. parryii (1)

Dist.: Alta, BC, NWT

Records: 1. Spencer 1966 (BC, NWT); 2.

Hilton and Mahrt 1971 (Alta)

Neohaematopinus marmotae Ferris, 1923

Hosts: Cynomys l. ludovicianus (2)

Marmota caligata okanagana (2)

M. flaviventris avara $(1,2)$

M. monax petrensis (2)

Mustela frenata nevadensis (2)

Dist.: BC, Sask

Records: 1. Holland 1938 (BC); 2. Spencer 1966

(BC, Sask)

Neohaematopinus sciurinus (Mjberg, 1910)

Syn.: Neohaematopinus antennatus semifasciatus Ferris, 1916

Hosts: Microtus pennsylvanicus (5)

Sciurus carolinensis (1)

S. c. pennsylvanicus (3)

Tamiasciurus hudsonicus $(2,4)$

T. h. columbiensis (3)

$T$. h. lanuginosus (3)

T. h. streatori (3)

Dist.: Alta, BC, Ont, Que

Records: 1. Judd 1954a (Ont); 2. Scholton et al. 1962 (Ont); 3. Spencer 1966 (BC); 4. Mahrt and Chai 1972 (Alta); 5. Gyorkos and Hilton 1982

(Que)

Neohaematopinus sciuropteri (Osborne, 1891)

Hosts: Eutamias amoenus affinus (3)

Glaucomys sabrinus (3)

G. s. alpinus (3)

G. s. columbiensis (3)

G. s. oregonensis $(1,3)$

Tamias striatus (2)

Dist.: Alta, BC, Ont

Records: 1. Spencer 1956 (Alta, BC); 2.

Scholten et al. 1962 (Ont); 3. Spencer 1966

(Alta, BC)
Polyplax alaskensis Ewing, 1927

Hosts: Clethrionomys rutilus (1)

Dist.: NB, NWT Microtus pennsylvanicus (2)

Records: 1. Mitchell and Behin 1965 (NWT); 2.

Whitaker and French 1982 (NB)

Polyplax auricularis Kellogg and Ferris, 1915

Host: Peromyscus maniculatus borealis

Dist.: Alta

Record: Spencer 1966

Polyplax spinulosa Burmeister, 1839

Syn.: Haematopinus spinulosus (Burmeister, 1839)

Hosts: Microtus oregoni serpens (5)

Rattus norvegicus $(1,2,3,4,5)$

R. r. rattus (5)

Rattus spp. (5)

Dist.: BC, Que

Records: 1. Whitehead 1934 (BC); 2. Spencer

1937 (BC), 3. 1939b (BC); 4. Firlotte 1948

(Que); 5. Spencer 1966 (BC)

Family LINOGNATHIDAE

Solenopotes ferrisi (Fahrenholz, 1919)

Syn.: Cervophthirius ferrisi

Linognathus ferrisi Fahrenholz, 1919

Hosts: Cervus canadensis (4) Odocoileus hemionus (6)

O. h. columbianus $(2,4)$

$O$. virginianus $(3,5,6)$

Odocoileus spp. (1)

Tamiasciurus hudsonicus columbianus (4)

Dist.: Alta, BC, Ont

Records: 1. Spencer 1938a (BC); 2. Cowan

1946 (BC); 3. Scholten et al. 1962 (Ont); 4.

Spencer 1966 (Alta, BC); 5. Watson and

Anderson 1975 (Ont); 6. Samuel et al. 1980

(Alta) 



\section{HOST-PARASITE LIST}



CLASS MAMMALIA

\section{ORDER ARTIODACTYLA}

Family ANTILOCAPRIDAE

Antilocapra a americana (Ord) Pronghorn

CHELICERATA

ACARI

Dermacentor andersoni (Alta)

Family BOVIDAE
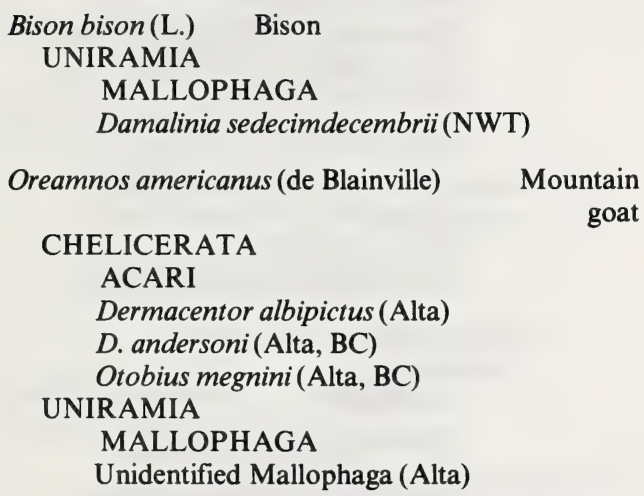

Oreamnos americanus missoulae (Allen)

CHELICERATA

ACARI

Dermacentor andersoni (BC)

UNIRAMIA

MALLOPHAGA

Damalinia oreamnidis (Alta)

Ovis canadensis Shaw Mountain sheep CHELICERATA

ACARI

Dermacentor albipictus (Alta)

D. andersoni (Alta, BC)

Otobius megnini (BC)

Psoroptes ovis (Alta)

Ovis canadensis californiana Douglas

CHELICERATA

ACARI

Dermacentor albipictus (BC)

Otobius megnini $(\mathrm{BC})$

UNIRAMIA

DIPTERA

Melophagus ovinus montanus (Alaska-

Yukon boundary)

MALLOPHAGA

Damalinia ovis (BC)

Ovis canadensis canadensis Shaw

CHELICERATA

ACARI

Dermacentor andersoni (BC)
Family CERVIDAE

Alces alces (L.) Moose

CHELICERATA

ACARI

Dermacentor albipictus (Alta, BC, Man, NB, NS, Ont, Que, Sask)

Dermacentor andersoni (Alta, BC, Ont)

UNIRAMIA

DIPTERA

Cephenemyia jellisoni (Ont)

C. phobifer (Ont)

Cephenemyia sp. (BC, Ont)

Alces alces americana (Clinton)

Syn.: Alces americanus

CHELICERATA

ACARI

Dermacentor albipictus (Alta, BC)

UNIRAMIA

DIPTERA

Cephenemyia jellisoni (Ont)

C. phobifera (Ont)

Cephenemyia spp. (BC)

Alces alces andersoni Peterson

CHELICERATA

ACARI

Dermacentor andersoni (Alta, BC)

Cervus elaphus L. Wapiti

CHELICERATA

ACARI

Dermacentor albipictus (Alta, BC, Que, Sask)

D. andersoni (Alta)

Otobius megnini (BC)

UNIRAMIA

DIPTERA

Cephenemyia jellisoni (Alta)

MALLOPHAGA

Mallophaga gen. spp. (Alta)

Cervus elaphus canadensis Erxleben

Syn.: Cervus canadensis Erxleben

CHELICERATA

ACARI

Dermacentor albipictus (Alta, BC)

UNIRAMIA

DIPTERA

Cephenemyia jellisoni (Alta)

MALLOPHAGA

Damalinia concavifrons (Alta, BC)

D. longicornis (Alta)

SIPHUNCULATA

Solenopotes ferrisi (Alta)

Cervus elaphus nelsoni Bailey

Syn.: Cervus canadensis nelsoni Bailey

UNIRAMIA

MALLOPHAGA

Damalinia concavifrons (Alta)

D. longicornis (Alta)

Odocoileus hemionus (Rafinesque) Mule deer CHELICERATA 
ACARI

Dermacentor albipictus (Alta, BC, Sask)

D. andersoni (BC)

Haemaphysalis leporis-palustris (Ont)

Ixodes pacificus $(\mathrm{BC})$

Otobius megnini (BC)

UNIRAMIA

DIPTERA

Cephenemyia apicata (Alta, BC)

C. jellisoni (Alta, BC)

C. trompe (Ont)

Lipoptena depressa $(\mathrm{BC})$

Neolipoptena ferrisi (BC)

MALLOPHAGA

Trichodectes parallelus (Alta)

Tricholipeurus lipeuroides (Alta)

T. parallelus (Alta, BC)

SIPHUNCULATA

Solenopotes ferrisi (Alta)

Odocoileus heminonus columbianus (Richardson)

CHELICERATA

ACARI

Dermacentor albipictus (BC)

D. andersoni $(\mathrm{BC})$

Ixodes californicus $(\mathrm{BC})$

I. pacificus $(\mathrm{BC})$

UNIRAMIA

DIPTERA

Cephenemyia jellisoni (BC)

Lipoptena depressa $(\mathrm{BC})$

Neolipoptena ferrisi (BC)

Phormia regina $(\mathrm{BC})$

MALLOPHAGA

Tricholipeurus lipeuroides $(\mathrm{BC})$

$T$. parallelus $(\mathrm{BC})$

Tricholipeurus spp. (BC)

SIPHONAPTERA

Pulex irritans (BC)

SIPHUNCULATA

Solenopotes ferrisi (BC)

Odocoileus hemionus hemionus (Rafinesque)

CHELICERATA

ACARI

Dermacentor albipictus (BC)

D. andersoni $(\mathrm{BC})$

UNIRAMIA

DIPTERA

Cephenemyia jellisoni (BC)

Lipoptena depressa $(\mathrm{BC})$

Neolipoptena ferrisi (BC)

MALLOPHAGA

Trichodectes tibialis (BC)

Tricholipeurus lipeuroides (Alta, BC)

T. parallelus $(\mathrm{BC})$

Odocoileus virginianus (Zimmermann)

CHELICERATA

White-tailed deer

ACARI

Dermacentor albipictus (Alta, Ont)

Haemaphysalis leporis-palustris (Ont)
Ixodes scapularis (Ont)

Otobius megnini (BC)

Otodectes cynotis (Alta)

Trombiculidae gen. spp. (Ont)

UNIRAMIA

DIPTERA

Cephenemyia phobifera(Ont)

C. trompe (Ont)

Cephenemyia spp. (Ont)

Lipoptena depressa $(\mathrm{BC})$

Neolipoptena ferrisi (BC)

MALLOPHAGA

Tricholipeurus lipeuroides (Alta, Ont)

T. parallelus (Alta, NS, Ont)

SIPHUNCULATA

Solenopotes ferrisi (Alta,Ont)

Odocoileus virginianus borealis Miller

UNIRAMIA

MALLOPHAGA

Tricholipeurus lipeuroides (Que)

Odocoileus spp.

UNIRAMIA

DIPTERA

Lipoptena depressa $(\mathrm{BC})$

MALLOPHAGA

Tricholipeurus parallelus (Ont)

SIPHUNCULATA

Solenopotes ferrisi (BC)

Rangifer tarandus (L.) Caribou

CHELICERATA

ACARI

Chorioptes texanus (NWT)

UNIRAMIA

DIPTERA

Cephenemyia trompe (Man, Nfld, NWT, Sask)

Oedemagena tarandi (Man, NWT)

Rangifer tarandus caribou (Gmelin)

Syn.: Rangifer caribou sylvestris Richardson

Syn.: Rangifer montanus Thompson-Seton

CHELICERATA

ACARI

Dermacentor albipictus (NS)

UNIRAMIA

DIPTERA

Cephenemyia phobifer (Sask)

C. trompe (Alta, BC)

Oedemagena tarandi (Alta)

Rangifer tarandus groenlandicus (L.)

UNIRAMIA

DIPTERA

Oedemagena tarandi (Alta, BC, NWT, YT)

CERVIDAE OF UNDETERMINED SPECIES

Coast deer

CHELICERATA

ACARI

Dermacentor albipictus (BC) 
Ixodes pacificus $(\mathrm{BC})$

I. ricinus $(\mathrm{BC})$

UNIRAMIA

DIPTERA

Lipoptena depressa $(\mathrm{BC})$

L. ferrisi (BC)

Deer

CHELICERATA

ACARI

Dermacentor albipictus (Alta, BC, NS)

D. andersoni (Alta, BC, Ont)

Ixodes cookei $(\mathrm{BC})$

Otobius megnini $(\mathrm{BC})$

UNIRAMIA

MALLOPHAGA

Tricholipeurus parallelus (Ont)

SIPHUNCULATA

Solenopotes ferrisi (BC)

ORDER CARNIVORA

Family CANIDAE

Alopex lagopus L. Arctic fox

UNIRAMIA

SIPHONAPTERA

Amalaraeus dissimilis (Alta, BC, NWT, YT)

Oropsylla alaskensis (NWT,YT)

Alopex lagopus lagopus $\mathrm{L}$.

Syn.: Alopex lagopus innuitus (Merriam)

UNIRAMIA

SIPHONAPTERA

Oropsylla alaskensis (NWT, YT)

O. arctomys (Alta, BC, Man, NB, NWT, Ont, Que, Sask)

Canis latrans Say Coyote

CHELICERATA

ACARI

Dermacentor albipictus (BC)

D. andersoni (Alta, BC)

Ixodes rugosus $(\mathrm{BC})$

I. sculptus (BC)

Sarcoptes scabiei (Alta)

UNIRAMIA

DIPTERA

Melophagus ovinus montanus $(\mathrm{BC})$

SIPHONAPTERA

Ceratophyllus garei (Alta, BC, Man, NWT,

Que, YT)

Chaetopsylla setosa (BC)

Megarthroglossus sicamus (BC)

Oropsylla arctomys (Alta, BC, Man, NB,

NWT, Que)

Pulex irritans (Alta, Man, PEI, Sask)

Thrassis acamantis (BC)

Canis latrans incolatus Hall

UNIRAMIA

MALLOPHAGA

Trichodectes canis $(\mathrm{BC})$

Tricholipeurus lipeuroides (BC)
Canis latrans lestes Merriam

UNIRAMIA

MALLOPHAGA

Trichodectes canis $(\mathrm{BC})$

Canis lupus L. Gray wolf

CHELICERATA

ACARI

Sarcoptes scabiei (Alta)

UNIRAMIA

MALLOPHAGA

Trichodectes canis (Ont)

Canis lupus columbianus Goldman

UNIRAMIA

MALLOPHAGA

Trichodectes canis $(\mathrm{BC})$

Canis spp.

UNIRAMIA

SIPHONAPTERA

Nearctopsylla brooksi (Alta, BC, YT)

Oropsylla rupestris (Alta)

Vulpes vulpes (L.) Red fox

CHELICERATA

ACARI

Dermacentor andersoni (Alta, BC)

Ixodes texanus (Ont)

Otodectes cynotis (Que)

Sarcoptes scabiei (Alta)

UNIRAMIA

SIPHONAPTERA

Amalaraeus dissimilis (NWT)

Cediopsylla i. inaequalis (Sask)

C. simplex (Ont)

Chaetopsylla lotoris (Ont)

Ctenocephalides canis (BC, Ont)

Epitedia w. wenmanni(Ont)

Euhoploplsyllus glacialis lynx (Alta, BC,

NWT, YT)

Megabothris abantis (Alta, BC)

Monopsyllus vison (Alta, BC, Man, Nfld, NS,

NWT, Ont, Que, Sask, YT)

Orchopeas c. caedens (Alta, BC, NWT, Sask,

YT)

O. c. durus (Alta, BC, NB, Nfld, NWT, Ont,

Que, Sask)

Oropsylla arctomys (Alta, BC, Man, NB,

Ont, Que, Sask)

Pulex irritans (Alta, Man, Sask)

Vulpes vulpes rubricosa Bangs

Syn.: Vulpes fulva rubricosa of Brown (1968).

UNIRAMIA

SIPHONAPTERA

Euhoplopsyllus glacialis lynx (NB)

Vulpes spp.

UNIRAMIA

SIPHONAPTERA

Amalaraeus dissimilis (NWT)

CANIDAE OF UNDETERMINED SPECIES

Fox

UNIRAMIA 
SIPHONAPTERA

Euhoplopsyllus glacialis lynx

(Alta,BC,NWT,YT)

Family FELIDAE

Lynx canadensis Kerr Lynx

UNIRAMIA

SIPHONAPTERA

Euhoplopsyllus glacialis lynx (Alta, BC, NB, NWT, YT)

Foxella ignota albertensis (Alta)

Monopsyllus thambus (Alta)

M. vison (Alta)

Opisodasys vesperalis $(\mathrm{BC})$

Orchopeas caedens durus (BC, Nfld, NWT)

Oropsylla halaskensis (NWT, YT)

Pulex irritans (Alta)

Trichopsylla setosus (BC)

T. thamba (Alta)

Lynx canadensis canadensis Kerr

UNIRAMIA

MALLOPHAGA

Felicola spenceri $(\mathrm{BC})$

Lynx rufus Rafinesque Bobcat

UNIRAMIA

SIPHONAPTERA

Euhoplopsyllus glacialis lynx (Alta, BC,

NWT, YT)

Opisodasys pseudarctomys (Alta, BC, Man,

NS, Ont, Que)

Orchopeas caedens durus (Alta, BC, NB,

Nfld, NWT, Ont, Que, Sask)

Tarsopsylla octodecimdentata colaradensis (Alta, BC, Man, NWT, Ont, Sask, YT)

Lynx rufus baileyi Merriam

Syn.: Lynx baileyi Merriam

UNIRAMIA

SIPHONAPTERA

Euhoplopsyllus glacialis lynx (Alta, BC, NWT, YT)

Lynx rufus fasciatus Rafinesque

Syn.: Lynx fasciatus Rafinesque

UNIRAMIA

SIPHONAPTERA

Euhoplopsyllus glacialis lynx (Alta, BC, NB, NWT)

Lynx spp.

\section{UNIRAMIA}

SIPHONAPTERA

Euhoplopsyllus glacialis lynx (Alta, BC, NB,

NWT, YT)

Megarthroglossus sicamus (BC)

Monopsyllus thambus (Alta)

Nearctopsylla brooksi (Alta, BC)

Orchopeas sexdentatus agilis (Alta, BC, YT)

Pulex irritans (Alta,Man)

\section{Family MUSTELIDAE}

Gulo luscus (L.) Wolverine

Syn.: Gulo gulo (L.)
Gulo gulo luscus of Holland (1949).

UNIRAMIA

SIPHONAPTERA

Caetopsylla setosa $(\mathrm{BC})$

Thrassis s. spenceri (BC)

Martes americana (Turton) American marten

Syn.: Mustela americana

CHELICERATA

ACARI

Listrophorus mustelae (BC)

UNIRAMIA

SIPHONAPTERA

Ceratophyllus lunatus tundrensis (NWT)

C. vison (Ont)

Epitedia w. wenmanni (Alta, BC, Man, NB, Nfld, NS, NWT, Ont, Que, Sask, YT)

Megabothris atrox (Ont)

Monopsyllus vison (Alta, BC, Man, Nfld, NS,

NWT, Ont, Que, Sask, YT)

Nearctopsylla brooksi (Alta, BC, YT)

$N$. g. genalis (NB, Nfld, Ont, Que)

N. hyrtaci $(\mathrm{BC})$

Orchopeas caedens (Alta)

O. c. caedens (Alta, BC, Man, Que, YT)

O. c. durus (Alta, BC, NB, Nfld, NWT, Ont,

Que, Sask)

O. sexdentatus agilis (Alta, BC, YT)

Oropsylla arctomys (Alta, BC, Man, NB,

NWT, Ont, Que, Sask)

Peromyscopsylla selenis (Alta, BC, Man,

NWT, Sask)

Rhadinopsylla arborea (Alta, BC)

$R$. fraterna (Alta, BC, Sask)

Tarsopsylla octodecimdentata coloradensis (Alta, BC, Man, NWT, Ont, Sask, YT)

Martes americana abietinoides Gray

CHELICERATA

ACARI

Lynxacarus mustelae (BC)

UNIRAMIA

SIPHONAPTERA

Nearctopsylla brooksi (Alta, BC, YT)

Martes americana actuosa (Osgood)

Syn.: Martes actuosa

Mustela americana actuosa

UNIRAMIA

SIPHONAPTERA

Orchopeas c. caedens (Alta, BC, Man, Que, YT)

O. c. durus (Alta, BC, NB, Nfld, NWT, Ont, Que, Sask)

Martes americana americana (Turton)

UNIRAMIA

SIPHONAPTERA

Ceratophyllus vison (Ont)

Hystrichopsylla dippiei (Ont)

Monopsyllus vison (Ont)

Nearctopsylla grahami (Ont)

Orchopeas caedens durus (Ont)

Oropsylla arctomys (Ont) 
Rhadinopsylla fraterna (Ont)

Tarsopsylla octodecimdentata coloradensis

(Ont)

Martes americana caurina (Merriam)

UNIRAMIA

SIPHONAPTERA

Monopsyllus ciliatus protinus (BC)

Nearctopsylla hyrtaci (BC)

Martes pennanti (Erxleben) Fisher

UNIRAMIA

SIPHONAPTERA

Oropsylla arctomys (Alta, BC, Man, NB,

NWT, Ont, Que, Sask)

Martes pennanti pennanti (Erxleben)

UNIRAMIA

SIPHONAPTERA

Oropsylla arctomys (Alta, BC, Man, NB,

NWT, Ont, Que, Sask)

Mephitis mephitis (Schreber) Striped skunk CHELICERATA

ACARI

Dermacentor andersoni (Alta, BC)

Hirstionyssus spp. (Ont)

Ixodes cookei (Ont)

I. kingi (BC, Sask)

I. marmotae $(\mathrm{BC})$

I. texanus (Ont)

Psocus spp. (Ont)

UNIRAMIA

MALLOPHAGA

Neotrichodectes mephitidis (Ont)

SIPHONAPTERA

Megabothris asio asio (Ont)

Monopsyllus vison (Alta, BC, Man, Nfld, NS,

NWT, Ont, Que, Sask, YT)

Thrassis acamantis (BC)

Mephitis mephitis mephitis (Schreber)

UNIRAMIA

SIPHONAPTERA

Megabothris quirini (Alta, BC, NWT, Ont)

Orchopeas caedens durus (BC, Nfld, NWT)

Oropsylla arctomys (Alta, BC, Man, NB,

Ont, Que, Sask)

Mephitis spp.

UNIRAMIA

SIPHONAPTERA

Oropsylla arctomys (Alta, BC, Man, NB,

NWT, Ont, Que, Sask)

Mustela erminea L. Ermine

Syn.: Mustela arctica (Merriam)

CHELICERATA

ACARI

Dermacentor andersoni (BC)

Ixodes cookei (NS)

I. kingi $(\mathrm{BC})$

UNIRAMIA

MALLOPHAGA
Stachiella kingi(Ont)

SIPHONAPTERA

Amalaraeus athabascae (BC, Man, Nfld,

NWT, Que, Sask)

A. dissimilis (Alta, BC, NWT, YT)

Catallagia decipiens (Alta, BC, Man, Sask,

YT)

Ceratophyllus lunatus tundrensis (Man, Nfld,

NWT, Que, YT)

C. vison (Ont)

Corrodopsylla c. curvata (Alta, BC, Man,

NB, Nfld, NS, NWT, Ont, Que, Sask, YT)

Dactylopsylla rara (Alta, Sask)

Epitedia w. wenmanni (Alta, BC, Man, NB,

Nfld, NS, NWT, Ont, Que, Sask, YT)

Euhoplopsyllus glacialis lynx (Alta, BC,

NWT, YT)

Foxella ignota albertensis (Alta, Sask)

F. i. recula (Man)

Megabothris abantis (Alta, BC)

M. a. asio (NB, Nfld, NS, Ont, PEI, Que)

M. a. megacolpus (Alta, BC, Man, NWT,

Ont, Que, Sask, YT)

$M$. atrox (Ont)

$M$. calcarifer gregsoni (NWT, YT)

M. groenlandicus (Can, Nfld, Que, YT)

M. quirini (Alta, BC, Man, Nfld, NWT, Ont, Que)

Monopsyllus vison (Alta, BC, Man, Nfld, NS, NWT, Ont, Que, Sask, YT)

Nearctopsylla brooksi (Alta, BC, YT)

N. g. genalis (NB, Nfld, Ont, Que)

N. g. hygini (Alta, Man, Sask)

N. hyrtaci (Alta, BC)

Orchopeas c. caedens (Alta, BC, Man, Que, YT)

O. c. durus (Alta, BC, NB, Nfld, NWT, Ont,

Que, Sask)

Oropsylla alaskensis (NWT)

Paramyscopsyllah. hamifer(Alta, Man, NWT,

Nfld, Ont, Que)

Rhadinopsylla difficilis (BC)

R. media (Alta,Que)

Mustela erminea anguinae Hall

UNIRAMIA

MALLOPHAGA

Trichodectes ermineae $(\mathrm{BC})$

SIPHONAPTERA

Monopsyllus ciliatus protinus (BC)

Mustela erminea arctica (Merriam)

UNIRAMIA

SIPHONAPTERA

Amalaraeus dissimilis (Alta, BC, NWT, YT)

Ceratophyllus lunatus tundrensis (NWT)

Foxella ignota albertensis (Alta, Sask)

Monopsyllus vison (Alta, BC, Man, Nfld, NS, NWT, Ont, Que, Sask, YT)

Nearctopsylla genalis hygini (Alta, Man, Sask)

Oropsylla alaskensis (NWT)

Mustela erminea bangsi Hall

UNIRAMIA

SIPHONAPTERA

Nearctopsylla genalis hygini (Alta,Man) 
Mustela erminea cicognanii Bonaparte

Syn.: Mustela cicognanii cicognanii

Putorius cicognani of Holland (1984).

\section{UNIRAMIA}

\section{SIPHONAPTERA}

Ceratophyllus lunatus tundrensis (Man, Nfld,

NWT, Que, YT)

Epitedia w. wenmanni (Alta, BC, Man, NB,

Nfld, NS, NWT, Ont, Que, Sask, YT)

Megabothris abantis (Alta, BC)

Monopsyllus vison (Alta, BC, Man, Nfld, NS,

NWT, Ont, Que, Sask, YT)

Nearctopsylla brooksi (Alta, BC, YT)

N. g. genalis (NB, Nfld, Ont, Que)

N. g. hygini (Alta, Man, Sask)

N. hyrtaci (Alta, BC)

Orchopeas c. caedens (Alta, BC, Man, Que,

YT)

O. c. durus (Alta, BC, NB, Nfld, NWT, Ont,

Que, Sask)

Peromyscopsylla h. hamifer (Alta, Nfld,

NWT, Ont, Que)

Mustela erminea fallenda Hall

UNIRAMIA

SIPHONAPTERA

Megabothris abantis (Alta, BC)

Monopsyllus ciliatus protinus (BC)

Orchopeas nepos (BC)

Mustela erminea invicta Hall

UNIRAMIA

SIPHONAPTERA

Epitedia w. wenmanni (Alta, BC, Man, NB,

Nfld, NS, NWT, Ont, Que, Sask, YT)

Malaraeus bitterootensis (Alta)

Monopsyllus vison (Alta, BC, Man, Nfld, NS,

NWT, Ont, Que, Sask, YT)

Nearctopsylla brooksi (Alta, BC, YT)

$N$. genalis hygini (Alta)

Orchopeas c. caedens (Alta, BC, Man, Que,

YT)

Mustela erminea richardsonii Bonaparte

Syn.: Mustela cicognani richardsonii

Putorius richardsonii of Holland (1984).

\section{UNIRAMIA}

MALLOPHAGA

Trichodectes ermineae $(\mathrm{BC})$

SIPHONAPTERA

Epitedia w. wenmanni (Alta, BC, Man, NB,

Nfld, NS, NWT, Ont, Que, Sask, YT)

Megabothris quirini (Alta, BC, Man, NB,

Nfld, NS, NWT, Ont, Que, Sask, YT)

Nearctopsylla brooksi (Alta, BC, YT)

N. g. genalis (NB)

N. g. hygini (Alta, Man, Sask)

Orchopeas c. caedens (Alta, BC, Man, Que,

YT)

O. c. durus (NB)

Mustela frenata Lichtenstein Long-tailed weasel

CHELICERATA

ACARI

Ixodes sculptus (Alta)

UNIRAMIA

\section{SIPHONAPTERA}

Catallagia decipiens (Alta, BC, Sask)

Epitedia w. wenmanni (Alta, BC, Man, NB,

Nfld, NS, NWT, Ont, Que, Sask, YT)

Foxella ignota albertensis (Alta, Sask)

F. i. recula $(\mathrm{BC})$

Megabothris abantis (Alta, BC, YT)

M. asio megacolpus (Alta, BC, Man, NWT,

Ont, Que, Sask, YT)

M. eumolpi (Alta, BC, Ont, Sask)

M. lucifer (Alta, BC, Sask)

Monopsyllus vison (BC)

Nearctopsylla brooksi (Alta, BC, YT)

N. hyrtaci (Alta, BC)

O. sexdentatus agilis (Alta, BC, YT)

Oropsylla idahoensis (Alta, BC, YT)

Mustela frenata arizonensis (Mearns)

Syn.: Mustela arizonensis (Mearns)

UNIRAMIA

SIPHONAPTERA

Foxella ignota recula $(\mathrm{BC})$

Monopsyllus w. wagneri (BC)

Orchopeas caedens durus (Alta, BC, NB,

Nfld, NWT, Ont, Que, Sask)

O. sexdentatus agilis (Alta, BC, YT)

Mustela frenata longicauda Bonaparte

Syn.: Mustela longicauda Bonaparte

UNIRAMIA

SIPHONAPTERA

Epitedia w. wenmanni (Alta, BC, Man, NB, Que)

Hystrichopsylla d. dippiei (Alta, Man, Ont)

H. gigas dippiei (Alta)

Malaraeus euphorbi (Alta, BC, Sask)

Megabothris lucifer (Alta, BC, Sask)

M. quirini (Alta, BC, NWT, Ont)

Nearctopsylla brooksi (Alta, BC)

N. hyrtaci (Alta, BC)

Neopsylla inopina (Alta, BC, Sask)

Opisocrostis labis (Alta, Sask)

Orchopeas caedens durus (Alta, BC, Man,

Que, YT)

O. sexdentatus agilis (Alta)

Oropsylla idahoensis (Alta)

O. labis (Alta)

$O$. rupestris (Alta, Man, Sask)

Rhadinopsylla fraterna (Alta, BC, Sask)

Thrassis b. bacchi (Alta, Man, Sask)

Trichopsylla abantis (Alta)

Mustela frenata nevadensis Hall CHELICERATA

\section{ACARI}

Dermacentor andersoni (Alta, BC)

Ixodes kingi (BC, Sask)

UNIRAMIA

SIPHONAPTERA

Megabothris lucifer (Alta, BC, Man, Sask)

Nearctopsylla brooksi (Alta, BC, YT)

Orchopeas caedens durus (BC)

O. sexdentatus agilis (Alta, BC, YT) 
SIPHUNCULATA

Neohaematopinus marmotae (BC)

Mustela frenata novaboracensis (Emmons)

UNIRAMIA

SIPHONAPTERA

Nearctopsylla g. genalis (NB, Nfld, Ont, Que) Opisodasys pseudarctomys (Alta, BC, Man, NS, Ont, Que)

Mustela frenata oribasus (Bangs)

UNIRAMIA

MALLOPHAGA

Trichodectes minutus (BC)

SIPHONAPTERA

Megabothris abantis (Alta, BC, YT)

Nearctopsylla brooksi (Alta, BC, YT)

Oropsylla idahoensis (Alta,BC)

Mustela nivalis rixosa (Bangs) Least weasel

Syn.: Mustela rixosa (Bangs)

Mustela rixosa rixosa (Bangs)

UNIRAMIA

MALLOPHAGA

Stachiella kingi (BC)

SIPHONAPTERA

Ceratophyllus garei (Alta, BC, Man, NWT,

Que, YT)

Megabothris calcarifer gregsoni (NWT, YT)

Mustela putorius L. Ferret

CHELICERATA

ACARI

Dermacentor andersoni (Alta,BC)

Mustela vison Schreber Mink
CHELICERATA
ACARI
Ixodes cookei (NS, Ont, Que)
I. pacificus (BC)
I. texanus (Ont)
UNIRAMIA

SIPHONAPTERA

Megabothris atrox (Alta, Man, Ont)

Monopsyllus vison (Alta, BC, Man, Nfld, NS,

NWT, Ont, Que, Sask, YT)

Nearctopsylla brooksi (Alta, BC, YT)

N. hyrtaci (BC)

Orchopeas caedens durus (Ont)

Trichopsylloides oregonensis (BC)

Mustela vison energumenos (Bangs)

UNIRAMIA

MALLOPHAGA

Trichodectes ermineae (BC)

SIPHONAPTERA

Dolichopsyllus stylosus (BC)

Hystrichopsylla d. dippiei (BC)

$H$. schefferi $(\mathrm{BC})$

Monopsyllus vison (Alta, BC, Man, Nfld, NS,

NWT, Ont, Que, Sask, YT)

Nearctopsylla brooksi (Alta, BC, YT)

N. hyrtaci (Alta, BC)
Orchopeas nepos (BC)

O. sexdentatus agilis (Alta, BC, YT)

Thrassis acamantis (BC)

Trichopsylloides oregonensis (BC)

Mustela vison vison Schreber

CHELICERATA

ACARI

Ixodes cookei (NS)

Mustela spp.

UNIRAMIA

SIPHONAPTERA

Ceratophyllus lunatus tundrensis (Man, Nfld,

NWT, Que, YT)

Ctenophthalmus p. pseudagyrtes (NB)

Ctenopsyllus hamifer (Alta)

Epitedia scapani $(\mathrm{BC})$

E. w. wenmanni (Alta, BC, Man, NB, Nfld,

NS, NWT, Ont, Que, Sask, YT)

Foxella ignota albertensis (Alta)

F. i. recula $(\mathrm{BC})$

Malaraeus telchinus (Alta, BC)

Megabothris abantis (Alta, BC, YT)

M. a. asio (Alta, NB, Nfld, NS, Ont, PEI,

Que)

M. a. megacolpus (Alta, Man)

M. atrox (Alta, Man, Ont)

M. groenlandicus (NWT, YT)

Megarthroglossus divisus exsecatus (BC)

Monopsyllus vison (Alta, BC, Man, Nfld, NS,

NWT, Ont, Que, Sask, YT)

Nearctopsylla brooksi (Alta, BC, YT)

N. g. genalis (NB, Ont)

N. g. hygini (Alta, Man, Sask)

N. hyrtaci (Alta, BC)

Neopsylla inopina (Alta, BC, Sask)

Orchopeas caedens durus (Alta, BC, NB,

Nfld, NWT, Ont, Que, Sask)

Oropsylla idahoensis (Alta, BC, YT)

$O$. rupestris (Alta, Sask)

Peromyscopsylla h. hamifer (Alta, BC, Man,

Nfld, NWT, Ont, Que, Sask)

Rhadinopsylla difficilis (BC)

R. fraterna (Alta, BC, Sask)

$R$. media (Alta, Que)

Trichopsylla a. asio (Alta)

T. atrox (Alta)

Spilogale putorius Merriam Spotted skunk

UNIRAMIA

SIPHONAPTERA

Orchopeas nepos (BC)

O. sexdentatus agilis $(\mathrm{BC})$

Spilogale putorius gracilis Merriam

Syn.: Spilogale gracilis latifrons Merriam Spilogale gracilis olympica of Holland (1949).

UNIRAMIA

MALLOPHAGA

Trichodectes osborni $(\mathrm{BC})$

SIPHONAPTERA

Atyphloceras m. multidentatus (BC) 
Hystrichopsylla d. dippiei (BC)

H. schefferi (BC)

Megarthroglossus p. procus (BC)

Monopsyllus ciliatus protinus (BC)

Rhadinopsylla sectilis goodi (BC)

Spilogale putorius latifrons Merriam

UNIRAMIA

SIPHONAPTERA

Monopsyllus ciliatus protinus (BC)

Spilogale putorius phenax Merriam

Syn.: Spilogale phenax Merriam

UNIRAMIA

SIPHONAPTERA

Ctenocephalides canis (BC, Ont)

Monopsyllus ciliatus protinus (BC)

Spilogale spp.

UNIRAMIA

SIPHONAPTERA

Ctenocephalides felis felis (BC)

Megarthroglossus p. procus $(\mathrm{BC})$

Taxidea taxus (Schreber) Badger

CHELICERATA

ACARI

Ixodes kingi (Alta)

UNIRAMIA

SIPHONAPTERA

Opiscrostis bruneri (Alta, Man, Sask, unspecified)

Oropsylla rupestris (Alta)

Pulex irritans (Alta,Man,Sask)

Taxidea taxus taxus (Schreber)

CHELICERATA

ACARI

Dermacentor andersoni (Alta, BC)

UNIRAMIA

SIPHONAPTERA

Opisocrostis labis (Alta, Sask)

Oropsylla arctomys (Alta, BC, Man, NB,

Ont, Que, Sask)

\section{MUSTELIDAE OF UNDETERMINED SPECIES}

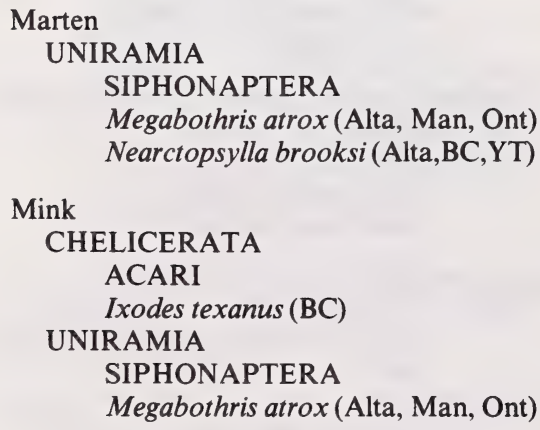

Marten

UNIRAMIA

SIPHONAPTERA

Megabothris atrox (Alta, Man, Ont)

Nearctopsylla brooksi (Alta,BC,YT)

Mink

CHELICERATA

ACARI

Ixodes texanus $(\mathrm{BC})$

UNIRAMIA

SIPHONAPTERA

Megabothris atrox (Alta, Man, Ont)

Mustelidae gen. spp.

CHELICERATA

ACARI
Ixodes texanus $(\mathrm{BC})$

UNIRAMIA

SIPHONAPTERA

Epitedia w. wenmanni (Alta, BC, Man, NB,

Nfld, NS, NWT, Ont, Que, Sask, YT)

Pine marten

UNIRAMIA

SIPHONAPTERA

Orchopeas c. caedens (Alta, BC, Man, Que, YT)

Short-tailed weasel

UNIRAMIA

SIPHONAPTERA

Monopsyllus vison (Alta, BC, Man, Nfld,

NS, NWT, Ont, Que, Sask, YT)

Skunk

UNIRAMIA

SIPHONAPTERA

Orchopeas sexdentatus agilis (Alta, BC, YT)

Thrassis acamantis (Alta, BC)

Weasel

CHELICERATA

ACARI

Dermacentor andersoni (Alta)

Ixodes hexagonus (BC)

I. marxi $(\mathrm{BC})$

I. texanus $(\mathrm{BC})$

UNIRAMIA

SIPHONAPTERA

Ceratophyllus lunatus tundrensis (Man, Nfld, NWT)

Epitedia scapani $(\mathrm{BC})$

Foxella ignota albertensis (Alta)

F. i. ignota (Man)

Megabothris asio megacolpus (Alta, BC,

Man, NWT, Ont, Que, Sask, YT)

Megarthroglossus divisus execatus (BC)

Monopsyllus ciliatus protinus (BC)

$M$. vison (Alta, BC, Man, Nfld, NS, NWT,

Ont, Que, Sask, YT)

M. w. wagneri (Alta, BC)

Nearctopsylla brooksi (Alta, BC, YT)

$N$. g. genalis (NB, Nfld, Ont, Que)

$N$. hyrtaci (Alta, BC)

Neopsylla inopina (Alta, BC, Sask)

Opisodasys k. keeni (Alta, BC)

$O$. vesperalis $(\mathrm{BC})$

Orchopeas c. caedens (Alta, BC, Man, NWT, Que, Sask, YT)

O. c. durus (Alta, BC, NB, Nfld, NWT, Ont,

Que, Sask)

O. nepos (BC)

O. sexdentatus agilis (Alta, BC, YT)

Oropsylla rupestris (Alta, Man, Sask, YT)

Thrassis acamantis (Alta, BC)

T. b. bacchi (Alta, Man, Sask) 
Family PROCYONIDAE

Procyon lotor (L.) Raccoon

CHELICERATA

ACARI

Dermacentor variabilis (NS)

Hirstionyssus sp. (NS)

Ixodes cookei (NS)

I. texanus (BC, Ont)

UNIRAMIA

SIPHONAPTERA

Chaetopsylla lotoris (Ont)

Monopsyllus vison (Alta, BC, Man, Nfld, NS,

NWT, Ont, Que, Sask,YT)

Procyon lotor vancouverensis Nelson and Goldman

UNIRAMIA

MALLOPHAGA

Trichodectes octomaculatus $(\mathrm{BC})$

Procyon spp.

UNIRAMIA

SIPHONAPTERA

Monopsyllus ciliatus protinus (BC)

Family URSIDAE

Ursus americanus Pallas Black bear

CHELICERATA

ACARI

Dermacentor variabilis (NS)

UNIRAMIA

MALLOPHAGA

Trichodectes pinquis euarctidos (BC, Ont)

SIPHONAPTERA

Chaetopsylla setosa $(\mathrm{BC})$

C. tuberculaticeps (Alta)

Oropsylla arctomys (Ont)

Ursus americanus americanus Pallas

CHELICERATA

ACARI

Dermacentor variabilis (NS)

Ursus americanus cinnamomum Audubon and

UNIRAMIA

Bachman

MALLOPHAGA

Trichodectes pinguis euarctidos (BC)

Ursus arctos L. Grizzly bear

Syn.: Ursus horribilis Ord

CHELICERATA

ACARI

Dermacentor andersoni (BC)

UNIRAMIA

SIPHONAPTERA

Chaetopsylla setosa (BC)

C. tuberculaticeps (Alta, BC)

Ursus spp.

CHELICERATA

ACARI

Dermacentor andersoni (Alta,BC)
URSIDAE OF UNDETERMINED SPECIES

Bears

CHELICERATA

ACARI

Dermacentor andersoni (Alta)

UNIRAMIA

SIPHONAPTERA

Oropsylla arctomys (Alta, BC, Man, NB,

Ont, Que, ask)

\section{ORDER CHIROPTERA}

Family MOLOSSIDAE

Eumops perotis californicus (Merriam) Greater

CHELICERATA

mastiff bat

ACARI

Ornithodoros spp. (BC)

UNIRAMIA

DIPTERA

Basilia spp. (BC)

Family VESPERTILIONIDAE

Eptesicus fuscus (Palisot de Beauvois) Big brown

\section{CHELICERATA}

ACARI

Spinturnix spp. (Ont)

UNIRAMIA

HEMIPTERA

Cimex pilosellus $(\mathrm{BC})$

SIPHONAPTERA

Myodopsylla insignis (Ont, Que)

M. palposa $(\mathrm{BC})$

Eptesicus fuscus fuscus (Palisot de Beauvois)

Syn.: Vespertilio fuscus Palisot de Beauvois

UNIRAMIA

HEMIPTERA

Cimex pilosellus $(\mathrm{BC})$

SIPHONAPTERA

Myodopsylla insignis (Alta, NS, Ont, Que,

YT)

M. palposa $(\mathrm{BC})$

Eptesicus fuscus pallidus Young

UNIRAMIA

HEMIPTERA

Cimex pilosellus $(\mathrm{BC})$

SIPHONAPTERA

Myodopsylla insignis (Alta, BC, Ont, Sask)

Lasionycteris noctivagans (Le Conte) Silver-haired

UNIRAMIA

HEMIPTERA

Cimex pilosellus (BC, Ont)

SIPHONAPTERA

Nycteridopsylla vancouverensis (BC)

Myotis keenii septentrionalis (Trovessart) Keen’s

UNIRAMIA 
SIPHONAPTERA

Myodopsylla insignis (Alta, BC, Ont, Sask)

Myotis lucifugus (Le Conte)

Little brown

bat

\section{CHELICERATA}

ACARI

Macronyssus crosbyi (PEI)

Pygmephorus makunkai (PEI)

Spinturnix americanus (PEI)

S. globosus (Alta)

UNIRAMIA

DIPTERA

Basilia forcipata $(\mathrm{BC})$

HEMIPTERA

Cimex pilasellus $(\mathrm{BC})$

SIPHONAPTERA

Myodopsylla gentilis (BC)

M. insignis (Alta, BC, NB, Ont, PEI, Que,

Sask, YT)

Myotis lucifugus alascensis Miller

UNIRAMIA

SIPHONAPTERA

Myodopsylla gentilis (BC)

Myotis lucifugus lucifugus (Le Conte)

CHELICERATA

ACARI

Liponyssus occidentalis (Ont)

Spinturnix spp. (Ont)

Steatonyssus occidentalis (Ont)

UNIRAMIA

HEMIPTERA

Cimex pilosellus (Ont)

SIPHONAPTERA

Myodopsylla gentilis (BC)

M. insignis (Man, Nfld, Ont)

Myotis lucifugus pernox Hollister

UNIRAMIA

SIPHONAPTERA

Myodopsylla insignis (Alta, NS, Ont, Que, YT)

Myotis yumanensis saturatus Miller Yuma bat

UNIRAMIA

SIPHONAPTERA

Myodopsylla gentilis (BC)

Myotis spp.

CHELICERATA

ACARI

Macronyssus crosbyi(NS)

Spinturnix sp. (NS)

UNIRAMIA

SIPHONAPTERA

Myodopsylla gentilis (BC)

M. insignis (Alta, BC, Man, NB, NS, Ont,

Que, Sask, YT)

\section{CHIROPTERA OF UNDETERMINED SPECIES}

Bat

\section{CHELICERATA}

ACARI

Liponyssus occidentalis (BC)

Liponyssus spp. (BC)

Ornithodoros kelleyi (Alta, Sask)

Ornithodoros spp. (unspecified)

Spinturnix spp. (BC)

UNIRAMIA

DIPTERA

Basilia forcipata (BC)

Trichobius spp. (BC)

SIPHONAPTERA

Myodopsylla gentilis (BC)

M. insignis (Alta, NS, Ont, Que, YT)

M. palposa $(\mathrm{BC})$

Brown bat

UNIRAMIA

SIPHONAPTERA

Myodopsylla insignis (Alta, BC, Ont, Sask)

M. palposa (BC)

Little brown bat

CHELICERATA

ACARI

Myobia caudata (Ont)

UNIRAMIA

DIPTERA

Basilia forcipata (unspecified)

HEMIPTERA

Cimex pilosellus $(\mathrm{BC})$

Silver-haired bat

UNIRAMIA

HEMIPTERA

Cimex pilosellus (BC)

ORDER INSECTIVORA

FAMILY SORICIDAE

Blarina brevicauda (Say)

Short-tailed

shrew

\section{CHELICERATA}

ACARI

Amorphacarus hengererorum (NB)

Androlaelaps fahrenholzi (NB, Ont)

Androlaelaps sp. (NB)

Bakerdania spp. (NB)

Blarinobia simplex (NB, Ont)

Comatacarus americanus (Ont)

Cyrtolaelaps spp. (NB)

Echinonyssus blarinae (NB)

E. isabellinus (NB)

E. talpae (NB)

Euhaemogamasus liponyssoides (Ont)

Eulaelaps stabularis (NB, NS, Ont)

Euryparasitus spp. (NB)

Euschoengastia blarinae (Ont)

Haemogamasus ambulans (NB, Ont)

H. liponyssoides (NB, Ont)

$H$. longitarsus (Ont) 
Haemagamasus sp. (NS)

Haemolaelaps glasgowi (Ont)

Hirstionyssus talpae (NS)

Hirstionyssus spp. (NS, Ont)

Ixodes angustus (NB, NS, Ont)

I. muris (NS)

Laelaps kochi(Ont)

Listrophorus spp. (Ont)

Miyatrombicula esoensis (NB)

Myobia simplex(Ont)

Myobiidae gen. spp. (Ont)

Myocoptes spp. (Ont)

Myonyssus jamesoni (Ont)

Neotrombicula microti (NS)

Orycteroxenus soricis (NB)

Protomyobia americana (NB)

P. claparedei (Ont)

Pygmephorus horridus (NB)

P. johnstoni (NB)

P. lutterloughae (NB)

P. moreohorridus (NB)

$P$. nidicolus (NB)

P. whitakeri (NB)

Pygmephorus spp. (NB)

Trombicula jamesoni (Ont)

UNIRAMIA

COLEOPTERA

Leptinus americanus (Ont)

SIPHONAPTERA

Atyphloceras bishopi(Ont)

Catallagia borealis (Alta, Ont)

Corrodopsylla c. curvata (Alta, BC, Man,

NB, Nfld, NS, NWT, Ont, Que, Sask, YT)

Ctenophthalmus p. pseudagyrtes (Alta, Man,

NB, NS, Ont, PEI, Que, Sask)

Doratopsylla blarinae (BC, NB, NS, Ont,

Que)

Epitedia w. wenmanni (Alta, BC, Man, NB,

Nfld, NS, NWT, Ont, Que, Sask, YT)

Hystrichopsylla tahavuana (NB, Ont)

Megabothris a. asio (Ont)

M. quirini (Alta, BC, Man, NB, Nfld, NS,

NWT, Ont, Que, Sask, YT)

Nearctopsylla genalis (Ont)

N. g. genalis (NB, Nfld, Ont, Que)

N. g. hygini (Alta, Man, Sask)

Nosopsyllus fasciatus (BC, Man, Nfld, NS,

Ont, Que)

Orchopeas leucopus (Ont)

Stenoponia americana (Man, NS, Ont, Que)

SIPHUNCULATA

Hoplopleura acanthopus (Ont)

Blarina brevicauda angusta Anderson

UNIRAMIA

SIPHONAPTERA

Corrodopsylla c. curvata (NB)

Ctenophthalmus p. pseudagyrtes (NB)

Peromyscopsylla catatina (NB)

Blarina brevicauda manitobensis Anderson

UNIRAMIA

\section{SIPHONAPTERA}

Ceratophyllus wagneri systaltus (Man)

Corrodopsylla c. curvata (Man)

Doratopsylla blarinae (Man)

Megabothris asio megacolpus (Alta, BC,

Man, NWT, Ont, Que, Sask, YT)

M. quirini (Man)

Monopsyllus wagneri systaltus (Man)

Nearctopsylla genalis hygini (Alta, Man,

Sask)

Peromyscopsylla catatina (Man)

Stenoponia americana (Man)

Blarina brevicauda pallida Smith

CHELICERATA

ACARI

Dermacentor variabilis (NS)

Ixodes angustus (NS)

UNIRAMIA

SIPHONAPTERA

Ctenophthalmus p. pseudagyrtes (NS)

Blarina brevicauda talpoides (Gapper)

UNIRAMIA

SIPHONAPTERA

Atyphloceras bishopi(Ont)

Corrodopsylla c. curvata (BC, Ont, Sask)

Ctenophthalmus p. pseudagyrtes (Alta, Man,

NB, NS, Ont, PEI, Que, Sask)

Doratopsylla blarinae (NB, NS, Ont, Que)

Hystricopsyllus gigas tahavauna (Ont)

H. tahavauna (Ont)

Orchopeas leucopus (Alta, BC, Man, NWT,

Ont, Sask, Que, YT)

Stenoponia americana (Man, NS, Ont, Que)

Blarina spp.

UNIRAMIA

SIPHONAPTERA

Corrodopsylla c. curvata (Alta, BC, Man,

NB, Nfld, NS, NWT, Ont, Que, Sask, YT)

Ctenophthalmus p. pseudagyrtes (Alta, Man,

NB, NS, Ont, PEI, Que, Sask)

Doratopsylla blarinae (NB, NS, Ont, Que)

Epitedia w. wenmanni (Alta, BC, Man, NB,

Nfld, NS, NWT, Ont, Que, Sask, YT)

Megabothris a. asio (NB, Nfld, NS, Ont, PEI, Que)

Nearctopsylla g. genalis (NB, Nfld, Ont, Que)

Orchopeas leucopus (Alta, BC, Man, NB,

Nfld, NS, NWT, Ont, Que, Sask)

Microsorex hoyi (Baird) Pygmy shrew CHELICERATA

ACARI

Amorphacarus hengererorum (NB)

Bakerdania sp. (NB)

Cyrtolaelaps sp. (NB)

Echinonyssus talpae (NB)

Haemogamasus ambulans (NB)

Ixodes angustus (NB)

Miyatrombicula esoensis (NB)

Orycteroxenus canadensis (NB) 
O. soricis (NB)

Protomyobia americana (NB)

Pygmephorus horridus (NB)

P. moreohorridus (NB)

UNIRAMIA

SIPHONAPTERA

Corrodopsylla c. curvata (Alta, BC, Man, NB, Nfld, NS, NWT, Ont, Que, Sask, YT)

Microsorex hoyi hoyi Elliot

UNIRAMIA

SIPHONAPTERA

Stenoponia americana (Man, NS, Ont, Que)

Microsorex spp.

UNIRAMIA

SIPHONAPTERA

Corrodopsylla c. curvata (Alta, BC, Man,

NB, Nfld, NS, NWT, Ont, Que, Sask, YT)

Sorex arcticus Kerr Arctic shrew

CHELICERATA

ACARI

Ixodes muris (NB)

UNIRAMIA

SIPHONAPTERA

Ceratophyllus wagneri systaltus (Man)

Corrodopsylla c. curvata (Man)

Monopsyllus wagneri systaltus (Alta, Man,

Ont, Sask)

Nearctopsylla g. genalis (NB)

N. g. hygini (Alta, Man, Sask)

Sorex arcticus arcticus Kerr

Syn.: Sorex richardsonii Bachman

UNIRAMIA

SIPHONAPTERA

Nearctopsylla genalis hygini (Alta, Man,

Sask)

N. hyrtaci (Alta, BC)

Sorex arcticus laricorum Jackson

UNIRAMIA

SIPHONAPTERA

Corrodopsylla c. curvata (Alta, BC, Man,

NB, Nfld, NS, NWT, Ont, Que, Sask, YT)

Sorex cinereus Kerr Masked shrew

CHELICERATA

ACARI

Amorphacarus hengererorum (NB)

Bakerdania sp. (NB)

Cyrtolaelaps sp. (NB)

Echinonyssus talpae (NB)

Eulaelaps stabularis (NS, Sask)

Euryparasitus sp. (NB)

Euschoengastia blarinae (Ont)

Glycephagidae gen. sp. (Ont)

Haemogamasus liponyssoides (NB)

Haemogamasus sp. (NS)

Ixodes angustus (NB)

I. marxi (PEI)

I. muris (PEI)
I. spinipalpis (Alta)

Miyatrombicula esoensis (NB)

Myobia sp. (NS)

Myobiidae gen. spp. (Ont)

Neotrombicula harperi (NB)

Orycteroxenus soricis (NB)

Protomyobia claparedei (NB)

Psorergates cinereus (Man, Ont)

Pygmephorus horridus (NB)

$P$. moreohorridus (NB)

P. whitakeri(NB)

UNIRAMIA

SIPHONAPTERA

Ceratophyllus wagneri systaltus (Man)

Corrodopsylla curvata (Ont)

C. c. curvata (Alta, BC, Man, NB, Nfld, NS,

NWT, Ont, Que, Sask, YT)

C. hamiltoni (NB)

Ctenophthalmus p. pseudagyrtes (Alta, Man, NB, NS, Ont, PEI, Que, Sask)

Epitedia w. wenmanni (Alta, BC, Man, NB,

NS, NWT, Ont, Que, Sask, YT)

Megabothris quirini (Alta, BC, Man, NB,

Nfld, NS, NWT, Ont, Que, Sask, YT)

Monopsyllus wagneri systaltus (Alta, Man,

Ont, Sask)

Nearctopsylla g. genalis (NB, Nfld, Ont, Que)

$N$. g. laurentina $(\mathrm{NB})$

N. hyrtaci (BC)

Orchopeas leucopus (Alta, BC, Man, NB,

Nfld, NS, NWT, Ont, Que, Sask)

Peromyscopsylla catatina (Man, Nfld, NS,

Ont)

P. h. hamifer (Alta, BC, Man, Nfld, NWT, Ont, Sask)

SIPHUNCULATA

Hoplopleura hesperomydis (Ont)

Sorex cinereus acadicus Gilpin

CHELICERATA

ACARI

Ixodes angustus (NS)

I. muris (NS)

UNIRAMIA

SIPHONAPTERA

Corrodopsylla c. curvata(NS)

Sorex cinereus cinereus Kerr

UNIRAMIA

SIPHONAPTERA

Corrodopsylla c. curvata (Alta, BC, Man, NB, Nfld, NS, NWT, Ont, Que, Sask, YT) Epitedia w. wenmanni (Man)

Megabothris quirini (Alta, BC, Man, NB,

Nfld, NS, NWT, Ont, Que, Sask, YT)

Orchopeas leucopus (Man)

Peromyscopsylla catatina (Man)

SIPHUNCULATA

Hoplopleura acanthopus (Ont)

Sorex cinereus haydeni Baird

UNIRAMIA

SIPHONAPTERA

Corrodopsylla c. curvata (BC, Ont, Sask) 
Sorex cinereus miscix Bangs

UNIRAMIA

SIPHONAPTERA

Corrodopsylla c. curvata (Que)

Nearctopsylla g. genalis (NB, Nfld, Ont, Que)

Sorex fumeus Miller Smoky shrew

CHELICERATA

ACARI

Amorphacarus hengererorum (NB)

Androlaelaps fahrenholzi (NB)

Bakerdania sp. (NB)

Cyrtolaelaps sp. (NB)

Echinonyssus talpae (NB)

Epitedia wenmanni (NB)

Euryparasitus sp. (NB)

Euschoengastia blarinae (Ont)

Glycyphagus hypudaei (NB)

Haemogamasus ambulans (NB)

$H$. liponyssoides (NB)

Ixodes angustus (NB, NS)

Miyatrombicula esoensis (NB)

Myonyssus jamesoni (Ont)

Orycteroxenus soricis (NB)

Protomyobia brevisetosa (NB)

Pygmephorus horridus (NB)

P. moreohorridus (NB)

P. whitakeri (NB)

Pygmephorus sp. (NB)

Xenoryctes nudus (NB)

UNIRAMIA

SIPHONAPTERA

Corrodopsylla c. curvata (Alta, BC, Man,

NB, Nfld, NS, NWT, Ont, Que, Sask, YT)

Ctenophthalmus p. pseudagyrtes (Alta, Man,

NB, NS, Ont, PEI, Que, Sask)

Epitedia w. wenmanni (NB)

Nearctopsylla g. genalis (NB, Nfld, Ont, Que)

Sorex gaspensis Anthony and Goodwin

CHELICERATA

ACARI

Amorphacarus hengererorum (NB)

Bakerdania sp. (NB)

Cyrtolaelaps sp. (NB)

Echinonyssus talpae (NB)

Euryparasitus sp. (NB)

Glycyphagus hypudaei (NB)

Haemogamasus ambulans (NB)

H. liponyssoides (NB)

Ixodes angustus (NB)

Miyatrombicula esoensis (NB)

Orycteroxenus soricis (NB)

Pygmephorus horridus (NB)

$P$. moreohorridus (NB)

P. nidicolus (NB)

$P$. whitakeri (NB)

Sorex palustris Richardson Water shrew

\section{CHELICERATA}

ACARI

Amorphacarus hengererorum (NB)

Cyrtolaelaps sp. (NB)
Echinonyssus talpae (NB)

Glycephagidae gen. spp. (Ont)

Glycyphagus hypudaei (NB)

Haemogamasus ambulans (NB)

Haemogamasus spp. (NS)

Hirstionysus spp. (Ont)

Ixodes angustus (NB)

Labidophorids (Ont)

Miyatrombicula esoensis (NB)

Myonyssus jamesoni (NB)

Orycteroxenus soricis (NB)

Protomyobia claparedei (NB)

Pygmephorus horridus (NB)

P. moreohorridus (NB)

UNIRAMIA

SIPHONAPTERA

Corrodopsylla curvata (Ont)

C. c. curvata (Alta, BC, Man, NB, Nfld, NS,

NWT, Ont, Que, Sask, YT)

C. c. obtusata (BC)

Megabothris asio megacolpus (Alta, BC,

Man, NWT, Ont, Que, Sask, YT)

Neartopsylla hyrtaci (Alta, BC)

Sorex trowbridgii Baird Trowbridge's shrew

UNIRAMIA

SIPHONAPTERA

Hystrichopsylla o. occidentalis (BC)

Sorex trowbridgii trowbridgii Baird

UNIRAMIA

SIPHONAPTERA

Epitedia scapani (BC)

Sorex vagrans Baird Vagrant shrew

Syn.: Sorex obscurus obscurus of Holland (1949).

Sorex obscurus setosus of Holland (1984).

CHELICERATA

ACARI

Ixodes soricis $(\mathrm{BC})$

UNIRAMIA

SIPHONAPTERA

Corrodopsylla c. curvata (Alta, BC, Man, NB, Nfld, NS, NWT, Ont, Que, Sask, YT) Nearctopsylla hyrtaci (Alta,BC)

Sorex vagrans obscurus Merriam

Syn.: Sorex obscurus Merriam

UNIRAMIA

SIPHONAPTERA

Ceratophyllus garei (Alta, BC, Man, NWT,

Que, YT)

Hystrichopsylla o. occidentalis (BC)

Nearctopsylla hyrtaci (Alta, BC)

Sorex vagrans vagrans Baird

CHELICERATA

ACARI

Ixodes soricis $(\mathrm{BC})$

Sorex vagrans vancouverensis Merriam

UNIRAMIA

SIPHONAPTERA

Corrodopsylla curvata obtusata (BC) 
Sorexspp.

CHELICERATA

ACARI

Ixodes angustus $(\mathrm{BC})$

I. soricis $(\mathrm{BC})$

COLEOPTERA

Leptinus americanus $(\mathrm{BC})$

UNIRAMIA

SIPHONAPTERA

Catallagia decipiens (Alta, BC, Man, Sask,

YT)

Corrodopsylla c. curvata (Alta, BC, Man,

NB, Nfld, NS, NWT, Ont, Que, Sask, YT)

C. c. obtusata (BC)

Corypsylla kohlsi (BC)

Doratopsylla blarinae (Ont)

Epitedia scapani $(\mathrm{BC})$

Hystrichopsylla d. dippiei (BC)

H. d. truncata $(\mathrm{BC})$

H. o. occidentalis $(\mathrm{BC})$

Malaraeus telchinus (BC)

Megabothris abantis (Alta, BC, YT)

M. a. asio (NB, Nfld, NS, Ont, PEI, Que)

Nearctopsylla genalis hygini (Alta, Man,

Sask)

N. hyrtaci (Alta, BC)

Nosopsyllus fasciatus (BC, Ont, Que, Sask)

Peromyscopsylla selenis (Alta, BC, Man,

NWT, Sask, YT)

\section{SORICIDAE OF UNDETERMINED SPECIES}

Shrew

CHELICERATA

ACARI

Ixodes angustus $(\mathrm{BC})$

UNIRAMIA

SIPHONAPTERA

Malaraeus telchinus (BC)

Water shrew

CHELICERATA

ACARI

Ixodes angustus $(\mathrm{BC})$

Family TALPIDAE

Condylura cristata (L.) Star-nosed mole CHELICERATA

\section{ACARI}

Androlaelaps fahrenholzi (NB)

Cyrtolaelaps spp. (NB)

Eadiea condylurae (NB)

Echinonyssus isabellinus (NB)

Eulaelaps stabularis (NB)

Euryparasitus spp. (NB)

Glycyphagus hypudaei (NB)

Haemogamasus ambulans (NB)

H. liponyssoides (NB)

Haemogamasus spp. (NS)

Hirstionyssus sp. (NS)

Ixodes angustus (NB)

Liponyssus spp. (Ont)
Orycteroxenus canadensis (NB)

O. soricis (NB)

Pygmephorus horridus (NB)

P. lutterloughae (NB)

P. moreohorridus (NB)

Xenoryctes nudus (NB)

UNIRAMIA

SIPHONAPTERA

Ctenophthalmus p. pseudagyrtes (Alta, Man, NB, NS, Ont, PEI, Que, Sask)

Epitedia w. wenmanni (Alta, BC, Man, NB,

Nfld, NS, NWT, Ont, Que, Sask, YT)

Megabothris a. asio (NB, Nfld, NS, Ont, PEI, Que)

Nearctopsylla g. genalis (NB, Nfld, Ont, Que)

Condylura cristata cristata (L.)

UNIRAMIA

SIPHONAPTERA

Ctenophthalmus p. pseudagyrtes (Alta, Man, Ont, Sask)

Condylura cristata nigra Smith

CHELICERATA

ACARI

Dermacentor variabilis (NS)

Ixodes angustus (NB)

UNIRAMIA

SIPHONAPTERA

Ctenophthalmus p. pseudagyrtes (Que)

Neurotrichus gibbsii (Baird) Shrew mole

UNIRAMIA

SIPHONAPTERA

Epitedia scapani $(\mathrm{BC})$

Hystrichopsylla o. occidentalis (BC)

Nearctopsylla jordani (BC)

Neurotrichus gibbsii gibbsii (Baird)

UNIRAMIA

SIPHONAPTERA

Catallagia charlottensis (BC)

Corrodopsylla curvata obtusata (BC)

Corypsylla jordani $(\mathrm{BC})$

Epitedia scapani (BC)

Nearctopsylla jordani (BC)

N. traubi $(\mathrm{BC})$

Neurotrichus spp.

UNIRAMIA

SIPHONAPTERA

Corypsylla jordani $(\mathrm{BC})$

C. ornata $(\mathrm{BC})$

Epitedia scapani $(\mathrm{BC})$

Nearctopsylla jordani (BC)

Parascalops breweri (Bachman) Hairy-tailed mole UNIRAMIA

SIPHONAPTERA

Ctenophthalmus p. pseudagyrtes (Alta, Man,

NB, NS, Ont, PEI, Que, Sask)

Doratopsylla blarinae (Ont)

Hystrichopsylla tahavuana (Que) 
Parascalops spp.

UNIRAMIA

SIPHONAPTERA

Ctenophthalmus p. pseudagyrtes (Alta, BC,

Man, NB, NS, Ont, PEI, Que, Sask)

Scalopus spp. Eastern mole

UNIRAMIA

SIPHONAPTERA

Nearctopsylla g. genalis (NB,Nfld,Ont,Que)

Scapanus aquaticus (L.) Western mole

UNIRAMIA

SIPHONAPTERA

Ctenophthalmus p. pseudagyrtes (Alta, Man,

NB, NS, Ont, PEI, Que, Sask)

Scapanus latimanus (Bachman) Broad-footed mole CHELICERATA

ACARI

Ixodes pacificus $(\mathrm{BC})$

Scapanus orarius True Coast mole

UNIRAMIA

SIPHONAPTERA

Epitedia scapani (BC)

Scapanus orarius orarius True

UNIRAMIA

SIPHONAPTERA

Epitedia scapani $(\mathrm{BC})$

Scapanus orarius schefferi Jackson

UNIRAMIA

SIPHONAPTERA

Catallagia charlottensis (BC)

C. s. sculleni (BC)

Corypsylla ornata $(\mathrm{BC})$

Epitedia scapani $(\mathrm{BC})$

Haemogamasus liponyssoides (BC)

Hystrichopsylla o. occidentalis (BC)

Nearctopsylla jordani (BC)

N. traubi $(\mathrm{BC})$

Scapanus townsendii (Bachman) Townsend's mole UNIRAMIA

SIPHONAPTERA

Corypsylla ornata $(\mathrm{BC})$

Epitedia scapani $(\mathrm{BC})$

Nearctopsylla traubi (BC)

Scapanus spp.

UNIRAMIA

SIPHONAPTERA

Catallagia charlottensis (BC)

Corypsylla ornata $(\mathrm{BC})$

Hystrichopsylla o. occidentalis (BC)

Nearctopsylla jordani (BC)

\section{TALPIDAE OF UNDETERMINED SPECIES}

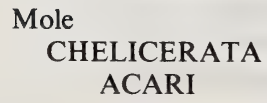

Mole

CHELICERATA

ACARI

Haemogamasus liponyssoides (BC)

UNIRAMIA

SIPHONAPTERA

Corypsylla ornata $(\mathrm{BC})$

Epitedia scapani (BC)

\section{ORDER LAGOMORPHA}

\section{Family LEPORIDAE}

Lepus americanus Erxleben Snowshoe rabbit CHELICERATA

\section{ACARI}

Cheyletiella parasitivorax (Ont)

Dermacentor variabilis (NS)

Haemaphysalis leporis-palustris (Alta, Man,

NB, NS, Ont, PEI, Sask)

Ixodes spinipalpus $(\mathrm{BC})$

UNIRAMIA

SIPHONAPTERA

Ceratophyllus vison (Ont)

Megabothris abantis (Alta, BC, YT)

Monopsyllus vison (Ont)

Lepus americanus bairdii Hayden

UNIRAMIA

SIPHONAPTERA

Euhoplopsyllus glacialis lynx (Alta, BC, NB, NWT)

Lepus americanus columbiensis Rhoads

UNIRAMIA

SIPHONAPTERA

Euhoplopsyllus glacialis lynx (Alta, BC, NB,

NWT)

Opisodasys vesperalis (BC)

Orchopeas sexdentatus agilis (Alta, BC, YT)

Lepus americanus dalli Merriam

Syn.: Lepus americanus macfarlani Merriam

UNIRAMIA

SIPHONAPTERA

Amalaraeus dissimilis (Alta, BC, NWT, YT)

Euhoplopsyllus glacialis lynx (Alta, BC,

NWT, YT)

Megabothris calcarifer gregsoni (NWT, YT)

Lepus americanus struthopus Bangs

CHELICERATA

ACARI

Haemaphysalis leporis-palustris (NS)

UNIRAMIA

SIPHONAPTERA

Euhoplopsyllus glacialis lynx (NB)

Lepus americanus virginianus Harlan

CHELICERATA

ACARI

Haemaphysalis leporis-palustris (Alta, Man,

NB, NS, Ont, PEI, Sask)

Neotrombicula basignata (NS)

UNIRAMIA

SIPHONAPTERA

Ceratophyllus vison (Ont) 
Megabothris abantis (Alta, BC, YT)

Monopsyllus ciliatus protinus (BC)

M. vison (Ont)

Lepus arcticus Ross Arctic hare

UNIRAMIA

SIPHONAPTERA

Euhoplopsyllus g. glacialis (NWT)

Lepus arcticus arcticus Ross

Syn.: Lepus glacialis Leach

UNIRAMIA

SIPHONAPTERA

Euhoplopsyllus g. glacialis (NWT)

Lepus capensis $\mathrm{L}$. Cape hare

Syn.: Lepus europaeus Pallas

CHELICERATA

ACARI

Haemaphysallis leporis-palustris (Ont)

UNIRAMIA

SIPHONAPTERA

Cediopsylla simplex (Ont)

SIPHUNCULATA

Haemodipsus spp. (Ont)

Lepus capensis hybridus Desmarest

CHELICERATA

ACARI

Haemaphysalis leporis-palustris (Ont)

UNIRAMIA

SIPHONAPTERA

Cediopsylla simplex (Ont)

SIPHUNCULATA

Haemodipsus ventricosus (Ont)

Haemodipsus spp. (Ont)

Lepus townsendii Bachman

White-tailed jack

rabbit

\section{CHELICERATA}

ACARI

Haemaphysalis leporis-palustris (Alta)

UNIRAMIA

SIPHONAPTERA

Pulex irritans (Man, Sask)

Lepus townsendii campanius Hollister

Syn.: Lepus campestris Bachman

CHELICERATA

ACARI

Dermacentor andersoni (Alta)

UNIRAMIA

SIPHONAPTERA

Oropsylla rupestris (Alta, Man, Sask)

Pulex irritans (Alta, Man, PEI)

Lepus spp.

UNIRAMIA

SIPHONAPTERA

Catallagia decipiens (Alta, BC, Man, Sask,

YT)

Cediopsylla i. inaequalis (Alta)

Euhoplopsyllus glacialis affinis (Sask)
E. g. lynx (Alta, BC, NWT, YT)

Hoplopsyllus spp. (Alta)

Hystrichopsylla d. dippiei (Alta)

Hystrichopsylla gigas dippiei (Alta)

Monopsyllus vison (Alta, BC, Man, Nfld, NS,

NWT, Ont, Que, Sask, YT)

Opisocrostis t. tuberculatus (Alta)

Pulex irritans (Alta, Man)

Sylvilagus floridanus (Allen) Eastern cottontail UNIRAMIA

SIPHONAPTERA

Cediopsylla simplex (Ont)

Epitedia w. wenmanni (Alta, BC, Man, NB,

Nfld, NS, NWT, Ont, Que, Sask, YT)

Euhoplopsyllus glacialis affinis (Sask)

Monopsyllus ciliatus protinus (BC)

Sylvilagus floridanus mearnsii Allen

UNIRAMIA

DIPTERA

Cuterebra spp. (Ont)

SIPHONAPTERA

Cediopsylla simplex (Ont)

Tamiophila grandis (Ont)

Sylvilagus nuttalii (Bachman) Nuttall's cottontail UNIRAMIA

SIPHONAPTERA

Cediopsylla i. inaequalis (Alta)

Sylvilagus nuttalii grangeri (Allen)

UNIRAMIA

SIPHONAPTERA

Cediopsylla i. inaequalis (Alta, Sask)

Megabothris quirini (Alta, BC, NWT, Ont)

Odontopsyllus dentatus (Alta, Sask)

Sylvilagus nuttallii nuttallii (Bachman)

UNIRAMIA

SIPHONAPTERA

Orchopeas sexdentatus agilis (BC)

Sylvilagus spp.

UNIRAMIA

SIPHONAPTERA

Cediopsylla i. inaequalis (Alta, Sask)

Odontopsyllus dentatus (Alta, Sask)

O. multispinosus (Alta)

\section{LEPORIDAE OF UNDETERMINED SPECIES}

Bush rabbit

UNIRAMIA

SIPHONAPTERA

Euhoplopsyllus glacialis lynx (Alta, BC, NB, NWT, YT)

Opisocrostis bruneri (unspecified)

Cottontail rabbit

UNIRAMIA

SIPHONAPTERA

Cediopsylla simplex (Ont)

Tamiophila grandis (Ont) 
Hare

CHELICERATA

ACARI

Haemaphysalis leporis-palustris (BC)

UNIRAMIA

SIPHONAPTERA

Euhoplopsylla glacialis lynx (Alta, BC, NB, NWT)

Jack rabbit

\section{CHELICERATA}

ACARI

Otobius megnini (Alta)

Rabbit

\section{CHELICERATA}

\section{ACARI}

Dermacentor andersoni (Alta, BC, Ont)

Haemaphysalis cinnabarina $(\mathrm{BC})$

H. leporis-palustris (BC, NB, NS, Que, Sask)

Ixodes angustus $(\mathrm{BC})$

I. dentatus spinipalpus $(\mathrm{BC})$

I. spinipalpus $(\mathrm{BC})$

Otobius megnini (Alta)

UNIRAMIA

DIPTERA

Cuterebra sp. (Ont)

SIPHONAPTERA

Euhoplopsylla glacialis lynx (Alta, BC, NWT, YT)

Monopsyllus w. wagneri (Alta, BC)

Lab rabbit

CHELICERATA

ACARI

Dermacentor andersoni (Alta, BC)

UNIRAMIA

SIPHUNCULATA

Snowshoe hare

Haemodipsus ventricosus $(\mathrm{BC})$

CHELICERATA

ACARI

Haemaphysalis leporis-palustris

Leporidae gen. spp.

\section{CHELICERATA}

ACARI

Dermacentor variabilis (NS)

\section{Family OCHOTONIDAE}

\section{Ochotona collaris (Nelson) Collared pika}

UNIRAMIA

SIPHONAPTERA

Amalaraeus dissimilis (Alta, BC, NWT, YT)

Ctenophyllus armatus (Alta, BC, YT)

Megabothris quirini (Alta, BC, Man, NB,

Nfld, NS, NWT, Ont, Que, Sask, YT)

Monopsyllus tolli (YT)

Opisocrostis t. tuberculatus (Alta, BC, Sask,

YT)

Oropsylla idahoensis (Alta, BC, YT)
Ochotona princeps (Richardson) Pika

CHELICERATA

ACARI

Ixodes angustus (BC)

I. dentatus spinipalpus $(\mathrm{BC})$

I. ochotonae (BC)

UNIRAMIA

SIPHONAPTERA

Amphalius runatus necopinus (Alta, BC)

Catallagia decipiens (Alta, BC, Sask)

Ctenophyllus armatus (Alta, BC, YT)

Megabothris abantis (Alta, BC)

Megarthroglossus spenceri (BC)

Monopsyllus ciliatus protinus (BC)

Orchopeas sexdentatus agilis (Alta, BC, YT)

Oropsylla idahoensis (Alta, BC, YT)

Peromyscopsylla ravalliensis (BC)

Rhadinopsylla difficilis (BC)

R. fraterna $(\mathrm{BC})$

Ochotona princeps brooksi Howell

CHELICERATA

ACARI

Ixodes ochotonae $(\mathrm{BC})$

UNIRAMIA

SIPHONAPTERA

Ctenophyllus armatus (Alta, BC, YT)

Monopsyllus e. eumolpi (Alta, BC, Man,

NWT, Ont, Sask, YT)

Orchopeas sexdentatus agilis (Alta, BC, YT)

Rhadinopsylla difficilis $(\mathrm{BC})$

Ochotona princeps brunnescens Howell

CHELICERATA

ACARI

Ixodes ochotonae (BC)

Ochotona princeps cuppes Bangs

UNIRAMIA

SIPHONAPTERA

Ctenophyllus armatus (Alta, BC)

Ochotona princeps fenisex Osgood

UNIRAMIA

SIPHONAPTERA

Ctenophyllus armatus (Alta, BC)

Monopsyllus eumolpi (Alta, BC, Ont, Sask)

SIPHUNCULATA

Hoplopleura acanthopus (BC)

Ochotona princeps princeps (Richardson)

UNIRAMIA

SIPHONAPTERA

Ctenophyllus armatus (Alta, BC)

Megabothris abantis (Alta, BC)

Oropsylla idahoensis (Alta, BC)

Ochotona spp.

CHELICERATA

ACARI

Ixodes angustus $(\mathrm{BC})$

I. spinipalpus $(\mathrm{BC})$ 
UNIRAMIA

SIPHONAPTERA

Amphalius runatus necopinus (Alta)

Catallagia decipiens (Alta, BC, YT)

Ctenophyllus armatus (Alta, BC, YT)

Euhoplopsylla glacialis lynx (Alta, BC, NWT,

YT)

Megabothris abantis (Alta, BC, YT)

Monopsyllus ciliatus protinus (BC)

M. e. eumolpi (Alta, BC, Man, NWT, Ont,

Sask, YT)

Orchopeas sexdentatus agilis (Alta, BC, YT)

\section{ORDER RODENTIA}

Family APLODONTIDAE

Aplodontia rufa Merriam Mountain beaver

UNIRAMIA

SIPHONAPTERA

Dolichopsyllus stylosus (BC)

Aplodontia rufa pacifica Merriam

Syn.: Aplodontia pacifica Merriam

CHELICERATA

COLEOPTERA

Leptinillus aplodontiae (BC)

Aplodontia rufa rufa (Rafinesque)

UNIRAMIA

SIPHONAPTERA

Dolichopsyllus stylosus (BC)

Epitedia scapani $(\mathrm{BC})$

Aplodontia spp.

CHELICERATA

COLEOPTERA

Leptinillus aplodontiae $(\mathrm{BC})$

Family CASTORIDAE

Castor canadensis Kuhl Beaver

CHELICERATA

ACARI

Ixodes cookei (Ont)

Prolabidocarpus canadensis (Ont)

Psorobia castoris (Ont)

COLEOPTERA

Leptinillus validus (BC, Hudson Bay Area,

Ont, Que)

Platypsyllus castoris (BC, Ont)

\section{CASTORIDAE OF UNDETERMINED SPECIES}

Beaver

CHELICERATA

COLEOPTERA

Leptinillus validus $(\mathrm{BC})$

Platypsyllus castoris (BC, Ont)
Family CAVIIDAE

Guinea pig

CHELICERATA

ACARI

Dermacentor andersoni $(\mathrm{BC})$

Ixodes pacificus $(\mathrm{BC})$

\section{FAMILY ERETHIZONTIDAE}

Erethizon dorsatum (L.) Porcupine CHELICERATA

ACARI

Dermacentor variabilis (NS)

Ixodes cookei (NS)

UNIRAMIA

MALLOPHAGA

Eutrichophilus setosus (NS, Ont)

SIPHONAPTERA

Ceratophyllus adjustus (BC)

Chaetopsylla setosa (BC)

Erithizon dorsatum dorsatum (L.)

CHELICERATA

ACARI

Ixodes cookei (NS)

Erethizon dorsatum epixanthium Brandt CHELICERATA

ACARI

Dermacentor andersoni (Alta)

Erithizon dorsatum nigrescens Allen

CHELICERATA

ACARI

Dermacentor andersoni (BC)

Ixodes spp. (BC)

UNIRAMIA

MALLOPHAGA

Eutrichophilus setosus (Alta, BC)

Erethizon sp.

CHELICERATA

ACARI

Dermacentor andersoni (BC)

Family GEOMYIDAE

Geomys sp.

UNIRAMIA

SIPHONAPTERA

Dactylopstlla ignota albertensis (Alta)

Thomomys talpoides (Richardson)

Northern pocket gopher

Syn.: Thomomys fuscus

Thomomys fuscus fuscus

UNIRAMIA

MALLOPHAGA

Geomydoecus sp. (Sask) 


\section{SIPHONAPTERA}

Dactylopsylla comis (BC)

D. rara (Alta, Sask)

Foxella ignota albertensis (Alta, Sask)

$F$. i. recula $(\mathrm{BC})$

Hystrichopsylla d. dippiei (Alta)

H. gigas dippiei (Alta)

Monopsyllus w. wagneri (Alta, BC)

Thomomys talpoides andersoni Goldman

UNIRAMIA

SIPHONAPTERA

Oropsylla idahoensis (Alta, BC)

Thomomys talpoides fuscus Merriam

UNIRAMIA

SIPHONAPTERA

Catallagia decipiens (Alta, BC, Man, Sask,

YT)

Foxella ignota albertensis (Alta)

SIPHUNCULATA

Hoplopleura acanthopus (BC)

Thomomys talpoides incensus Goldman

UNIRAMIA

SIPHONAPTERA

Catallagia decipiens (Alta, BC, Sask)

Foxella ignota recula $(\mathrm{BC})$

Thomomys spp.

UNIRAMIA

SIPHONAPTERA

Foxella ignota albertensis (Alta, Sask)

F. i. recula $(\mathrm{BC})$

Megabothris abantis (Alta, BC, YT)

Oropsylla idahoensis (Alta, BC, YT)

O. rupestris (Alta)

Rhadinopsylla fraterna (Alta, BC, Sask)

Thrassis petiolatus $(\mathrm{BC})$

Family HETEROMYIDAE

Dipodomys ordii Woodhouse

Ord's kangaroo

UNIRAMIA

SIPHONAPTERA

Monopsyllus wagneri systaltus (Alta, Man,

Ont, Sask)

Dipodomys ordii terrasus Hoffmeister

UNIRAMIA

SIPHONAPTERA

Meringis parkeri (Sask)

Perognathus parvus laingi Anderson

Great basin

UNIRAMIA

SIPHUNCULATA

Fahrenholzia pinnata $(\mathrm{BC})$

Perognathus parvus lordi (Gray)

Syn.: Perognathus l. lordi (Gray)

UNIRAMIA

SIPHONAPTERA
Meringis shannoni (BC)

SIPHUNCULATA

Fahrenholzia pinnata $(\mathrm{BC})$

Family MURIDAE

Clethrionomys gapperi (Vigors)

Gapper's red-

backed mouse

Syn.: Evotomys gapperi (Bailey)

Evotomys saturatus of Holland (1984).

\section{CHELICERATA}

ACARI

Androlaelaps fahrenholzi (NB)

Androlaelaps sp. (NS)

Cyrtolaelaps sp. (NB)

Dermacarus hypudaei septentrionalis (Que)

D. variabilis (Man, NS)

Echinonyssus isabellinus (NB)

Echinonyssus sp. (NB)

Eulaelaps stabularis (NB, NS)

Euschoengastia blarinae (NB)

Euschoengastia sp. (NS)

Glycyphagidae gen. spp. (Ont)

Glycyphagus hypudaei (NB)

Haemaphysalis leporis-palustris (NS)

Haemogamasus ambulans (NB, NS)

H. reidi $(\mathrm{NB})$

Haemogamasus spp. (NS)

Haemolaelaps glasgowi (Ont)

Hirstionyssus sp. (NS)

Hyperlaelaps sp. (NS)

Ixodes angustus (NB, NS)

I. muris (PEI)

Ixodes spp. (Ont)

Labidophorids (Ont)

Laelaps kochi (Ont)

Laelaps sp. (NS)

Listrophoridae gen. spp. (Ont)

Listrophorus mexicanus (NB, Que)

Macronyssus crosbyi (NS)

Miyatrombicula esoensis (NB)

Myobia spp. (Que)

Myobiidae gen. spp. (Ont)

Myocoptes j. japonensis (NB)

M. squamosus (NB)

Myocoptes spp. (Que)

Neotrombicula harperi (NB)

N. microti (NB)

Pygmephorus horridus (NB)

Radfordia lemnina (NB)

Radfordia spp. (Que)

Sarcoptidae gen. sp. (Ont)

Trichoecius tenax (NB)

Trombicula spp. (NS, Que)

Trombiculidae gen. spp. (Ont)

UNIRAMIA

SIPHONAPTERA

Amalaraeus athabascae (Alta, BC, Man,

Nfld, NWT, Que, Sask)

A. dissimilis (Alta, BC, NWT, YT)

Amphipsylla sibirica pollionis (Alta, BC, Nfld, YT)

Atyphloceras bishopi (Man, Nfld, Ont, Que) 
Catallagia borealis (Man, NB, Ont, Que)

C. charlottensis (Alta, BC)

C. dacenkoi fulleri (Alta, BC, Man, NWT, YT)

C. decipiens (Alta, BC, Man, Sask, YT)

C. jellisoni (Alta, BC, YT)

Ceratophyllus gallinae (Man)

C. wagneri systaltus (Man)

Corrodopsylla c. curvata (Alta, BC, Man,

NB, Nfld, NS, NWT, Ont, Que, Sask, YT)

Ctenophthalmus p. pseudagyrtes (Alta, BC,

Man, NB, NS, Ont, PEI, Que, Sask)

Ctenopsyllus selenis (Alta, BC)

Delotelis hollandi (Alta, BC)

D. telegoni (Alta, BC)

Epitedia w. wenmanni (Alta, BC, Man, NB,

Nfld, NS, NWT, Ont, Que, Sask, YT)

Hystrichopsylla d. dippiei (Man)

H. d. truncata $(\mathrm{BC})$

H. o. occidentalis (Alta, BC)

Malaraeus telchinus (Alta, BC)

Megabothris abantis (Alta, BC, YT)

M. asio (Ont)

M. a. asio (NB, Nfld, NS, Ont, PEI, Que)

M. a. megacolpus (Alta, BC, Man, NWT,

Ont, Que, Sask, YT)

M. quirini (Alta, BC, Man, NB, Nfld, NS,

NWT, Ont, Que, Sask, YT)

Monopsyllus thambus (Alta, BC, Nfld, NWT,

Que, YT)

M. vison (Alta, BC, Man, Nfld, NS, NWT,

Ont, Que, Sask, YT)

M. wagneri systaltus (Alta, Man, Ont, Sask)

Nearctopsylla g. genalis (NB)

Nopsylla inopina (Alta, BC, Sask)

Opisodasys k. keeni (Alta, BC)

$O$. pseudarctomys (Alta, Ont)

Orchopeas caedens durus (NB)

O. leucopus (Man)

O. sexdentatus agilis (Alta, BC, YT)

Peromyscopsylla catatina (Man, NB, Nfld, NS, Ont, Que)

P. h. hamifer (Alta, BC, Man, Nfld, NWT, Ont, Sask)

$P$. selenis (Alta, BC, Man, NWT, Sask, YT)

Rhadinopsylla arborea (Alta, BC)

$R$. fraterna (BC, Sask)

$R$. media (Alta, Que)

Stenoponia americana (Alta, Man, NB, NS,

Ont, Que)

Trichopsylla quirini (Alta)

SIPHUNCULATA

Hoplopleura acanthopus (Ont)

$H$. hesperomydis (Que)

\section{Clethrionomys gapperi athabascae (Preble)}

UNIRAMIA

SIPHONAPTERA

Amalaraeus athabascae (Alta, BC, Man,

Nfld, NWT, Que, Sask)

A. dissimilis (Alta, BC, NWT, YT)

Amphipsylla sibirica pollionis (Alta)
Catallagia dacenkoi fulleri (Alta, BC, Man, NWT, YT)

Epitedia w. wenmanni (Alta, BC, Man, NB, Nfld, NS, NWT, Ont, PEI, Que, Sask, YT) Megabothris asio megacolpus (Alta, BC, Man, NWT, Ont, Que, Sask, YT) M. quirini (Alta, BC, Man, NB, Nfld, NS, NWT, Ont, Que, Sask, YT)

Peromyscopsylla selenis (Alta, BC, Man, NWT, Sask, YT)

Rhadinopsylla linta (BC, NWT, YT)

Clethrionomys gapperi cascadensis Booth

UNIRAMIA

SIPHONAPTERA

Catallagia charlottensis (BC)

Clethrionomys gapperi caurinus (Bailey)

UNIRAMIA

SIPHONAPTERA

Catallagia charlottensis (BC)

Hystrichopsylla o. occidentalis (BC)

Clethrionomys gapperi gapperi (Vigors)

UNIRAMIA

SIPHONAPTERA

Megabothris quirini (Alta, BC, Nfld, NWT, Ont, Que)

Orchopeas leucopus (Alta, BC, Man, NWT, Ont, Que, Sask, YT)

Clethrionomys gapperi gaspeanus Anderson UNIRAMIA

SIPHONAPTERA

Ctenophthalmus p. pseudagyrtes (NB)

Megabothris quirini (NB)

Peromyscopsylla catatina (NB)

Clethrionomys gapperi loringi (Bailey)

UNIRAMIA

SIPHONAPTERA

Amphipsylla sibirica pollionis (Alta)

Atyphloceras bishopi(Man)

Catallagia borealis (Man)

C. decipiens (Alta, BC)

Ctenophthalmus p. pseudagyrtes (Alta, BC, Man, NB, NS, Ont, PEI, Que, Sask)

Epitedia w. wenmanni (Alta, BC, Man, NB, Nfld, NS, NWT, Ont, Que, Sask, YT)

Hystrichopsylla d. dippiei (Alta, BC, Man)

Megabothris asio megacolpus (Alta, BC,

Man, NWT, Ont, Que, Sask, YT)

M. quirini (Alta, BC, Man, NB, Nfld, NS,

NWT, Ont, Que, Sask, YT)

Monopsyllus vison (Alta, BC, Man, Nfld, NS, NWT, Ont, Que, Sask, YT)

$M$. wagneri systaltus (Alta, Man, Ont, Sask)

Orchopeas leucopus (Alta, BC, Man, NB,

Nfld, NS, NWT, Ont, Que, Sask)

Peromyscopsylla catatina (Man, Nfld, NS,

Ont)

P. h. hamifer (Man)

P. selenis (Alta, Man, NWT, Sask, YT) 
Scaphiopsylla bishopi (Man)

Stenoponia americana (Man, NS, Ont, Que)

Clethrionomys gapperi ochraceus (Miller)

UNIRAMIA

SIPHONAPTERA

Catallagia borealis (Man, NB, Ont, Que)

Ctenophthalmus p. pseudagyrtes (NB)

Epitedia w. wenmanni (NB)

Orchopeas leucopus (NB)

Peromyscopsylla catatina (NB)

Stenoponia americana $(\mathrm{NB})$

Clethrionomys gapperi pallescens Hall and Cockrum CHELICERATA

ACARI

Dermacentor variabilis (NS)

Haemaphysalis leporis-palustris (NS)

Ixodes angustus (NS)

Clethrionomys gapperi proteus (Bangs)

UNIRAMIA

SIPHONAPTERA

Amalaraeus athabascae (Alta, BC, Man,

Nfld, NWT, Que, Sask)

Atyphloceras bishopi (Nfld, Man, Ont, Que)

Catallagia borealis (Ont, Que)

Epitedia w. wenmanni (Alta, BC, Man, NB,

Nfld, NS, NWT, Ont, Que, Sask, YT)

Megabothris a. asio (NB, Nfld, NS, Ont, PEI, Que)

M. quirini (Alta, BC, Man, NB, Nfld, NS,

NWT, Ont, Que, Sask, YT)

Peromyscopsylla catatina (Man, Nfld, NS,

Ont, Que)

P. h. hamifer (Alta, Nfld, NWT, Ont, Que)

Rhadinopsylla media (Alta, Que)

Clethrionomys gapperi saturatus (Rhoads)

Syn.: Evotomys gapperi saturatus Rhoads

UNIRAMIA

SIPHONAPTERA

Amalaraeus dissimilis (Alta, BC, NWT)

Amphipsylla sibirica pollionis (Alta)

Catallagia charlottensis (Alta)

C. decipiens (Alta, BC, Sask)

Ctenophthalmus p. pseudagyrtes (Alta)

Delotelis telegoni (Alta)

Hystrichopsylla dippiei truncata $(\mathrm{BC})$

Malaraeus telchinus (BC)

Megabothris abantis (Alta, BC)

M. quirini (Alta, BC, NWT, Ont)

Nearctopsylla hyrtaci (BC)

Neopsylla inopina (Alta)

Orchopeas sexdentatus agilis (BC)

Peromyscopsylla selenis (Alta, BC, Man,

NWT, Sask)

Trichopsylla quirini (Alta)

SIPHUNCULATA

Hoplopleura acanthopus (BC)

Clethrionomys gapperi ungava (Bailey)

Syn.: Evotomys ungava Bailey
UNIRAMIA

SIPHONAPTERA

Amalaraeus athabascae (Alta, BC, Man,

Nfld, NWT, Que, Sask)

Epitedia w. wenmanni (Alta, BC, Man, NB,

Nfld, NS, NWT, Ont, Que, Sask, YT)

Megabothris asio (Nfld)

M. a. asio (NB, Nfld, NS, Ont, PEI, Que)

M. quirini (Alta, BC, Man, NB, Nfld, NS,

NWT, Ont, Que, Sask, YT)

Clethrionomys rutilus Pallas Northern red-backed

CHELICERATA

ACARI

Haemogamasus ambulans (NWT)

Hirstionyssus isabellinus (NWT)

Laelaps clethrionomydis (NWT)

Myocoptes spp. (NWT)

UNIRAMIA

SIPHONAPTERA

Amalaraeus dissimilis (Alta, BC, NWT, YT)

Amphipsylla sibirica pollionis (Alta, BC,

Nfld, YT)

Catallagia dacenkoi fulleri (Alta, BC, Man,

NWT, YT)

Corrodopsylla c. curvata (Alta, BC, Man,

NB, Nfld, NS, NWT, Ont, Que, Sask, YT)

Epitedia w. wenmanni (Alta, BC, Man, NB,

Nfld, NS, NWT, Ont, Que, Sask, YT)

Megabothris asio megacolpus (Alta, BC,

Man, NWT, Ont, Que, Sask, YT)

$M$. calcifer gregsoni (NWT, YT)

M. groenlandicus (Man, Nfld, NWT, Que,

YT)

M. quirini (Alta, BC, Man, NB, Nfld, NS,

NWT, Ont, Que, Sask, YT)

Monopsyllus thambus (Alta, BC, Nfld, NWT,

Que, YT)

M. vison (Alta, BC, Man, Nfld, NS, NWT,

Ont, Que, Sask, YT)

Orchopeas leucopus (Alta, BC, Man, NB,

Nfld, NS, NWT, Ont, Que, Sask)

Peromyscopsylla h. hamifer (Alta, BC, Man,

Nfld, NWT, Ont, Sask)

$P$. selenis (Alta, BC, Man, NWT, Sask, YT)

SIPHUNCULATA

Polyplax alaskensis (NWT)

Clethrionomys rutilus dawsoni (Merriam)

Syn.: Clethrionomys dawsoni (Merriam)

Clethrionomys dawsoni dawsoni (Merriam)

UNIRAMIA

SIPHONAPTERA

Amalaraeus dissimilis (Alta, BC, NWT, YT)

Amphipsylla sibirica pollionis (Alta, BC,

Nfld, YT)

Catallagia dacenkoi fulleri (Alta, BC, Man,

NWT, YT)

C. decipiens (Alta, BC, Man, Sask, YT)

C. jellisoni (Alta, BC, YT)

Ceratophyllus garei (Alta, BC, Man, NWT,

Que, YT) 
C. lunatus tundrensis (NWT, Que)

Corrodopsylla c. curvata (Alta, BC, Man,

NB, Nfld, NS, NWT, Ont, Que, Sask, YT)

Epitedia w. wenmanni (Alta, BC, Man, NB,

Nfld, NS, NWT, Ont, Que, Sask, YT)

Hystrichopsylla o. occidentalis (BC)

Megabothris asio megacolpus (Alta, BC,

Man, NWT, Ont, Que, Sask, YT)

$M$. calcarifer gregsoni (NWT, YT)

M. quirini (Alta, BC, Man, NB, Nfld, NS,

NWT, Ont, Que, Sask, YT)

Monopsyllus vison (Alta, BC, Man, Nfld, NS,

NWT, Ont, Que, Sask, YT)

Peromyscopsylla selenis (Alta, BC, Man,

NWT, Sask, YT)

Rhadinopsylla linta (BC, NWT, YT)

Clethrionomys spp.

CHELICERATA

ACARI

Ixodes angustus (Alta)

UNIRAMIA

SIPHONAPTERA

Amalaraeus athabascae (BC, Man, Nfld,

NWT, Que, Sask)

A. dissimilis (Alta, BC, NWT, YT)

Amphipsylla sibirica pollionis (Alta, BC,

Nfld, YT)

Atyphloceras bishopi (Man, Nfld, Ont, Que)

Catallagia charlottensis (BC)

C. dacenkoi fulleri (Alta, BC, Man, YT)

C. decipiens (Alta, BC, Man, Sask, YT)

C. sculleni chamberlini $(\mathrm{BC})$

Ceratophyllus garei (Alta, BC, Man, NWT,

Que, YT)

Corrodopsylla c. curvata (Alta, BC, Man,

NB, Nfld, NS, NWT, Ont, Que, Sask, YT)

Ctenophthalmus p. pseudagyrtes (Alta, BC,

Man, NB, NS, Ont, PEI, Que)

Delotelis hollandi (Alta, BC)

Hystrichopsylla o. occidentalis (Alta, BC)

Malaraeus euphorbi (Alta, BC, NWT, Sask)

M. telchinus (Alta, BC)

Megabothris abantis (Alta, BC, YT)

M. acerbus (Man, Ont)

M. asio megacolpus (Alta, BC, Man, NWT,

Ont, Que, Sask, YT)

$M$. calcarifer gregsoni (NWT, YT)

M. quirini (Alta, BC, Man, NB, Nfld, NS,

NWT, Ont, Que, Sask, YT)

Monopsyllus vison (Alta, BC, Man, Nfld, NS, NWT, Ont, Que, Sask, YT)

$M$. wagneri systaltus (Alta, Man, Ont, Sask)

Opisodasys k. keeni (Alta, BC)

Orchopeas caedens durus (Alta, BC, NB,

Nfld, NWT, Ont, Que, Sask)

O. leucopus (Alta, BC, Man, NB, Nfld, NS,

NWT, Ont, Que, Sask)

Peromyscopsylla catatina (Man, Nfld, NS,

Ont)

P. hesperomys adelpha (Alta, Sask)

$P$. selenis (Alta, BC, Man, NWT, Sask, YT)
Rhadinopsylla arborea (Alta, BC)

R. linta (BC, NWT, YT)

Stenoponia americana (Man, NS, Ont, Que)

Dicrostonyx groenlandicus (Traill) Collared

CHELICERATA

ACARI

Haemogamasus ambulans (NWT)

Hirstionyssus isabellinus (NWT)

Laelaps alaskensis (NWT)

Pygmephorus spp. (NWT)

UNIRAMIA

SIPHONAPTERA

Megabothris calcarifer gregsoni (NWT, YT)

M. groenlandicus (NWT)

SIPHUNCULATA

Hoplopleura acanthopus (NWT)

Dicrostonyx groenlandicus kilangmiutuk Anderson and Rand

UNIRAMIA

SIPHONAPTERA

Megabothris groenlandicus (NWT)

Dicrostonyx groenlandicus richardsoni Merriam

Syn.: Dicrostonyx torquatus richardsoni Merriam UNIRAMIA

SIPHONAPTERA

Ceratophyllus lunatus tundrensis (NWT, Que)

Megabothris asio megacolpus (Man)

M. groenlandicus (Man, Nfld, YT)

Dicrostonyx hudsonius (Pallas) Labrador collared

UNIRAMIA

SIPHONAPTERA

Ceratophyllus garei (Alta, BC, Man, NWT,

Que, YT)

C. lunatus tundrensis (NWT, Que)

Megabothris asio megacolpus (Que)

M. groenlandicus (Man, Nfld, NWT, Que, YT)

M. quirini (Alta, BC, Man, NB, Nfld, NWT, Ont, Que, Sask, YT)

Dicrostonyx spp.

CHELICERATA

ACARI

Listrophorus dicrostonyx (NWT)

Myocoptes japonensis canadensis (Ont)

UNIRAMIA

SIPHONAPTERA

Ceratophyllus lunatus tundrensis (Man, Nfld,

NWT, Que, YT)

Megabothris asio megacolpus (Que)

M. calcarifer gregsoni (NWT, YT)

M. groenlandicus (Nfld, Que, YT)

M. quirini (Alta, BC, Man, NB, Nfld, NS,

NWT, Ont, Que, Sask, YT)

Oropsylla alaskensis (NWT, YT) 
Lagurus curtatus (Cope) Sagebrush vole UNIRAMIA

SIPHONAPTERA

Catallagia decipiens (Alta, BC, Man, Sask, YT)

Megabothris c. clantoni (BC)

Monopsyllus wagneri systaltus (Alta, Man, Ont, Sask)

Peromyscopsylla hesperomys adelpha (Alta, Sask)

Rhadinopsylla fraterna (BC, Sask)

Lemmus sibiricus Kerr Brown lemming

Syn.: Lemmus trimucronatus (Richardson)

UNIRAMIA

SIPHONAPTERA

Amalaraeus dissimilis (Alta, BC, NWT, YT)

Amphipsylla sibirica pollionis (Alta, BC,

Nfld, YT)

Megabothris calcarifer gregsoni (NWT, YT)

M. groenlandicus (Man, Nfld, NWT, Que,

YT)

M. quirini (Alta, BC, Man, NB, Nfld, NS,

NWT, Ont, Que, Sask, YT)

Rhadinopsylla linta (BC, NWT, YT)

Lemmus sibiricus trimucronatus (Richardson)

Syn.: Lemmus t. trimucronatus (Richardson)

UNIRAMIA

SIPHONAPTERA

Catallagia dacenkoi fulleri (Alta, BC, Man,

YT)

Megabothris groenlandicus (Nfld, NWT,

Que, YT)

SIPHUNCULATA

Hoplopleura acanthopus (NWT)

Lemmus spp.

UNIRAMIA

SIPHONAPTERA

Megabothris calcarifer gregsoni (NWT, YT)

M. groenlandicus (Man, Nfld, YT)

Microtus chrotorrhinus (Miller) Rock vole CHELICERATA

ACARI

Androlaelaps fahrenholzi (NB)

Bakerdania sp. (NB)

Cyrtolaelaps sp. (NB)

Echinonyssus isabellinus (NB)

Eulaelaps stabularis (NB)

Euryparasitus sp. (NB)

Euschoengastia blarinae (NB)

E. setosa (NB)

Glycyphagus hypudaei (NB)

Haemogamasus ambulans (NB)

$H$. liponyssoides (NB)

Ixodes angustus (NB)

Laelaps alaskensis (NB)

L. kochi (NB)

Listrophorus mexicanus (NB)

Miyatrombicula esoensis (NB)

Myocoptes j. japonensis (NB)
M. squamosus (NB)

Neotrombicula harperi (NB)

Orycteroxenus canadensis (NB)

O. soricis (NB)

Pygmephorus horridus (NB)

Radfordia hylandia (NB)

R. lemnina $(\mathrm{NB})$

Radfordia sp. (NB)

Trichoecius tenax (NB)

Trichoecius spp. (NB)

UNIRAMIA

SIPHONAPTERA

Atyphloceras bishopi (Man, Nfld, Ont, Que)

Catallagia borealis (NB)

Ctenophthalmus p. pseudagyrtes (NB)

Epitedia w. wenmanni (Alta, BC, Man, NB,

Nfld, NS, NWT, Ont, Que, Sask, YT)

Megabothris quirini (NS)

Peromyscopsylla catatina (Man, NB, Nfld, NS, Ont)

Microtus chrotorrhinus chrotorrhinus (Miller)

UNIRAMIA

SIPHONAPTERA

Orchopeas leucopus (Alta, BC, Man, NWT,

Ont, Que, Sask, YT)

Peromyscopsylla catatina (NB, Ont)

Microtus longicaudus (Merriam) Long-tailed vole Syn.: Microtus longicaudus mordax (Merriam) UNIRAMIA

SIPHONAPTERA

Amalaraeus dissimilis (Alta, BC, NWT, YT)

Amphipsylla sibirica pollionis (Alta, BC,

Nfld, YT)

Catallagia decipiens (Alta, BC, Man, Sask,

YT)

Delotelis hollandi (Alta, BC)

D. telegoni (Alta, BC)

Epitedia w. wenmanni (Alta, BC, Man, NB,

Nfld, NS, NWT, Ont, Que, Sask, YT)

Hystrichopsylla dippiei truncata $(\mathrm{BC})$

H. o. occidentalis (BC)

Malaraeus telchinus (Alta, BC)

Megabothris abantis (Alta, BC, YT)

$M$. calcarifer gregsoni (NWT, YT)

M. quirini (Alta, BC, Man, NB, Nfld, NS,

NWT, Ont, Que, Sask, YT)

Megarthroglossus d. divisus (BC)

Monopsyllus vison (Alta, BC, Man, Nfld, NS,

NWT, Ont, Que, Sask, YT)

Opisodasys k. keeni (Alta, BC)

Orchopeas sexdentatus agilis (BC)

Oropsylla idahoensis (Alta, BC)

Peromyscopsylla h. hamifer (Alta, BC, Man,

Nfld, NWT, Ont, Que, Sask)

P. selenis (Alta, BC, Man, NWT, Sask, YT)

Rhadinopsylla arborea (Alta, BC)

SIPHUNCULATA

Hoplopleura acanthopus (BC)

Microtus longicaudus macrurus Merriam UNIRAMIA 


\section{SIPHONAPTERA}

Catallagia charlottensis (BC)

Hystrichopsylla o. occidentalis (Alta, BC)

Megabothris abantis (Alta, BC, YT)

Peromyscopsylla selenis (Alta, BC, Man,

NWT, Sask, YT)

Microtus longicaudus vellerosus Allen

Syn.: Microtus vellerosus Allen

UNIRAMIA

SIPHONAPTERA

Amalaraeus dissimilis (Alta, BC, NWT, YT)

Catallagia dacenkoi fulleri (Alta, BC, Man,

YT)

Megabothris quirini (Alta, BC, Man, NB,

Nfld, NS, NWT, Ont, Que, Sask, YT)

Peromyscopsylla selenis (Alta, BC, Man,

NWT, Sask, YT)

SIPHUNCULATA

Hoplopleura acanthopus (BC)

Microtus miurus Osgood Singing vole

UNIRAMIA

SIPHONAPTERA

Amalaraeus dissimilis (Alta, BC, NWT, YT)

Megabothris calcarifer gregsoni (NWT, YT)

M. groenlandicus (Man, Nfld, NWT, Que, Y

Microtus montanus canescens Bailey Montane vole Syn.: Microtus nanus canescens Bailey

UNIRAMIA

SIPHONAPTERA

Catallagia decipiens (Alta, BC, Man, Sask, YT)

Delotelis hollandi (Alta, BC)

Epitedia w. wenmanni (Alta, BC, Man, NB,

Nfld, NS, NWT, Ont, Que, Sask, YT)

Hystrichopsylla dippiei truncata (BC)

Malaraeus telchinus (Alta, BC)

Megabothris lucifer (Alta, BC, Man, Sask)

Rhadinopsylla difficilis $(\mathrm{BC})$

SIPHUNCULATA

Hoplopleura acanthopus (BC)

Microtus ochrogaster (Wagner) Prairie vole

Syn.: Pedomys ochrogaster Baird

UNIRAMIA

SIPHONAPTERA

Ctenophthalmus p. pseudagyrtes (Alta, BC, Man, NB, NS, Ont, PEI, Que, Sask)

Megabothris asio megacolpus (Alta, BC,

Man, NWT, Ont, Que, Sask, YT)

M. quirini (Alta, BC, Man, NB, Nfld, NS,

NWT, Ont, Que, Sask, YT)

Monopsyllus wagneri systaltus (Alta, Man,

Ont, Sask)

Microtus ochrogaster minor (Merriam)

Syn.: Microtus minor of Hopkins and Rothschild (1962)

UNIRAMIA

SIPHONAPTERA

Ctenophthalmus p. pseudagyrtes (Alta, BC,
Man, NB, NS, Ont, PEI, Que, Sask)

Epitedia w. wenmanni (Alta, BC, Man, NB, Nfld, NS, NWT, Ont, Que, Sask, YT)

Hystrichopsylla d. dippiei (Alta, BC, Man, Ont)

Megabothris lucifer (Alta, BC, Man, Sask)

Stenoponia americana (Alta, Man, Que)

Microtus oeconomus (Pallas) Tundra vole UNIRAMIA

SIPHONAPTERA

Amalaraeus dissimilis (Alta, BC, NWT, YT)

Amphipsylla sibirica pollionis (Alta, BC,

Nfld, YT)

Catallagia dacenkoi fulleri (Alta, BC, Man, YT)

Corrodopsylla c. curvata (Alta, BC, Man,

NB, Nfld, NS, NWT, Ont, Que, Sask, YT)

Hystrichopsylla o. occidentalis (BC)

Megabothris asio megacolpus (Alta, BC,

Man, NWT, Ont, Que, Sask, YT)

$M$. calcarifer gregsoni (NWT, YT)

M. quirini (Alta, BC, Man, NB, Nfld, NS,

NWT, Ont, Que, Sask, YT)

Peromyscopsylla ostsibirica longiloba (YT)

Microtus oeconomus macfarlani Merriam

UNIRAMIA

SIPHONAPTERA

Monopsyllus e. eumolpi (Alta, BC, Man,

NWT, Ont, Sask, YT)

Microtus oeconomus operarius (Nelson)

Syn.: Microtus operarius

UNIRAMIA

SIPHONAPTERA

Amalaraeus dissimilis (Alta, BC, NWT, YT)

Megabothris quirini (Alta, BC, Man, NB,

Nfld, NS, NWT, Ont, Que, Sask, YT)

Peromyscopsylla selenis (Alta, BC, Man,

NWT, Sask, YT)

Microtus oregoni (Bachman) Creeping vole

UNIRAMIA

DIPTERA

Cuterebra spp. (BC)

Microtus oregoni serpens Merriam

Syn.: Microtus serpens Merriam

CHELICERATA

ACARI

Leptinus americanus (BC)

UNIRAMIA

SIPHONAPTERA

Atyphloceras m. multidentatus (BC)

Catallagia charlottensis (BC)

Corrodopsylla curvata obtusata (BC)

Corypsylla ornata $(\mathrm{BC})$

Dasypsyllus gallinulae perpinnatus (BC)

Delotelis hollandi (Alta, BC)

Epitedia wenmanni (BC)

Hystrichopsylla o. occidentalis (Alta, BC)

Megabothris abantis (Alta, BC, YT) 
Monopsyllus ciliatus protinus (BC)

$M$. wagneri ophidius $(\mathrm{BC})$

Nearctopsylla traubi (BC)

Opisodasys k. keeni (Alta, BC)

Peromyscopsylla hesperomys pacifica (BC)

P. selenis (Alta, BC, Man, NWT, Sask, YT)

Rhadinopsylla sectilis goodi $(\mathrm{BC})$

SIPHUNCULATA

Hoplopleura acanthopus (BC)

Polyplax spinulosa (BC)

Microtus pennsylvanicus (Ord) Meadow vole CHELICERATA

ACARI

Allothrombium sp. (NS)

Androlaelaps fahrenholzi (NB, Sask)

Androlaelaps sp. (NS)

Dermacentor variabilis (Man, NS)

Echinonyssus isabellinus (NB)

Eulaelaps stabularis (NS, Sask)

Euryparasitus sp. (NB)

Euschoengastia spp. (NS, Sask)

Glycyphagidae gen. spp. (Ont)

Glycyphagus hypudaei (NB)

Haemaphysalis leporis-palustris (PEI)

Haemogamasus ambulans (NB)

Haemogamasus sp. (NS)

Hirstionyssus sp. (NS, Ont)

Hyperlaelaps sp. (NS)

Ixodes angustus (NS)

I. muris (NB, NS, Ont, PEI)

Laelaps alaskensis (NB, Ont)

L. kochi (NB, Ont)

Laelaps sp. (NS)

Listrophoridae gen. spp. (Ont)

Listrophorus mexicanus (NB)

L. m. squamiferus (NWT)

Miyatrombicula esoensis (NB)

Myobia spp. (Que)

Myobiidae gen. sp. (Ont)

Myocoptes j. japonensis (NB)

M. squamosus (NB)

Neotrombicula basignata (NS)

$N$. harperi (NB)

N. microti (NS)

Ololaelaps sp. (NS)

Protomyobia brevisetosa (NB)

Psorergates canadensis (Man, Ont)

Pygmephorus moreohorridus (NB)

Radfordia hylandi (NB)

Radfordia spp. (Que)

Trichoecius tenax (NB)

UNIRAMIA

SIPHONAPTERA

Amalaraeus athabascae (Alta, BC, Man,

Nfld, NWT, Que, Sask)

$A$. dissimilis (Alta, BC, NWT, YT)

Amphipsylla sibirica pollionis (Alta, BC,

Nfld, YT)

Atyphloceras bishopi (Man, Nfld, Ont, Que)

Catallagia borealis (Man)

C. dacenkoi fulleri (Alta, BC, Man, NWT, YT)
C. decipiens (Alta, BC, Man, Sask, YT)

C. jellisoni (Alta, BC, YT)

Corrodopsylla c. curvata (Alta, BC, Man,

NB, Nfld, NS, NWT, Ont, Que, Sask, YT)

Ctenophthalmus p. pseudagyrtes (Alta, BC,

Man, NB, NS, Ont, PEI, Que, Sask)

Epitedia w. wenmanni(NS)

Hystrichopsylla d. dippiei (Alta, BC, Man,

Sask)

H. d. truncata $(\mathrm{BC})$

Megabothris abantis (Alta, BC, YT)

M. asio (NB, Ont)

M. a. $a \operatorname{sio}$ (NB, Nfld, NS, Ont, PEI, Que)

M. a. megacolpus (Ont)

M. calcarifer gregsoni (NWT, YT)

M. groenlandicus (Man, Nfld, YT)

M. quirini (Alta, BC, Man, NB, Nfld, NS,

NWT, Ont, Que, Sask, YT)

Monopsyllus vison (Alta, BC, Man, Nfld, NS,

NWT, Ont, Que, Sask, YT)

$M$. wagneri systaltus (Alta, Man, Ont, Sask)

Opisocrostis bruneri (Alta, Man, Sask)

Opisodasys pseudarctomys (Alta, BC, Man,

NS, Ont, Que)

Orchopeas leucopus (Alta, BC, Man, NB,

Nfld, NS, NWT, Ont, Que, Sask, YT)

Peromyscopsylla catatina (Man, Nfld, NS,

Ont)

P. h. hamifer (Alta, BC, Man, Nfld, NWT,

Ont, Que, Sask)

P. h. markworthi (Ont)

P. selenis (Alta, BC, Man, NWT, Sask, YT)

Polyplax alaskensis (NB)

Rhadinopsylla fraterna (BC, Sask)

Thrassis petiolatus (Alta, BC)

SIPHUNCULATA

Hoplopleura acanthopus (Ont, Sask)

Neohaematopinus semifasciatus (Que)

Polyplax alaskensis (NB)

Microtus pennsylvanicus acadicus Bangs

CHELICERATA

ACRAI

Dermacentor variabilis (NS)

Haemaphysalis leporis-palustris (NS)

Ixodes angustus (NS)

I. muris (NS)

Microtus pennsylvanicus drummondii (Audubon and Bachman)

Syn.: Microtus drummondii (Audubon and Bachman)

UNIRAMIA

SIPHONAPTERA

Amalaraeus athabascae (BC, Man, Nfld,

NWT, Que, Sask)

A. dissimilis (Alta, BC, NWT, YT)

Amphipsylla sibirica pollionis (Alta, BC,

Nfld, YT)

Catallagia dacenkoi fulleri (Alta, BC, Man,

NWT, YT)

C. decipiens (Alta, BC, Sask) 
C. jellisoni (Alta, BC)

Ctenophthalmus p. pseudagyrtes (Alta, Man,

Ont, Sask)

Ctenopsyllus selenis (BC)

Delotelis telegoni (BC)

Epitedia w. wenmanni (Alta, BC, Man, NB,

Nfld, NS, NWT, Ont, Que, Sask, YT)

Foxella ignota albertensis (Man)

Hystrichopsylla dippiei truncata (BC)

Megabothris abantis (Alta, BC, YT)

M. asio (NWT)

M. a. megacolpus (BC, Man, NWT, Sask)

$M$. calcarifer gregsoni (NWT, YT)

M. lucifer (Alta, BC, Sask)

M. quirini (Alta, BC, Man, NB, Nfld, NS,

NWT, Ont, Que, Sask, YT)

Monopsyllus e. eumolpi (BC)

Orchopeas leucopus (Alta, BC, Man, NB,

Nfld, NS, NWT, Ont, Que, Sask)

Peromyscopsylla catatina (Man)

P. h. hamifer (Alta, BC, Man, Nfld, NWT,

Ont, Sask)

P. selenis (BC, Man, NWT, Sask, YT)

Rhadinopsylla fraterna (Alta, BC, Sask)

Trichopsylla lucifer (Alta)

SIPHUNCULATA

Hoplopleura acanthopus (BC)

Microtus pennsylvanicus enixus Bangs

Syn.: Microtus enixus Bangs

CHELICERATA

ACARI

Ixodes muris (Nfld)

UNIRAMIA

SIPHONAPTERA

Epitedia w. wenmanni (Nfld)

Megabothris asio (Nfld)

M. a. asio (Nfld)

M. quirini (Nfld)

Peromyscopsylla h. hamifer (Alta, BC, Man,

Nfld, NWT, Ont, Que, Sask)

Microtus pennsylvanicus insperatus (Allen)

UNIRAMIA

SIPHONAPTERA

Megabothris quirini (Alta, BC, NWT, Ont)

Microtus pennsylvanicus labradorius Bailey

UNIRAMIA

SIPHONAPTERA

Megabothris asio megacolpis (Alta, BC, Man,

NWT, Ont, Que, Sask)

M. quirini (Alta, BC, Man, NB, Nfld, NS,

NWT, Ont, Que, Sask, YT)

Peromyscopsylla h. hamifer (Alta, BC, Man,

Nfld, NWT, Ont, Que, Sask)

Microtus pennsylvanicus pennsylvanicus (Ord)

CHELICERATA

ACARI

Androlaelaps fahrenholzi(Ont)

Laelaps kochi(Ont)

L. muris (Ont)

Listrophorus leuckarti (Ont)
UNIRAMIA

SIPHONAPTERA

Atyphloceras bishopi (Ont, Que)

Ctenophthalmus p. pseudagyrtes (Ont, Que)

Epitedia w. wenmanni (Alta, BC, Man, NB,

NWT, Ont, Que, Sask)

Megabothris a. asio (Que)

M. quirini (Alta, BC, NWT, Ont)

Nosopsyllus fasciatus (Que)

Orchopeas leucopus (Alta, BC, Man, NWT,

Ont, Que, Sask, YT)

Peromyscopsylla h. hamifer (Que)

SIPHUNCULATA

Hoplopleura acanthopus (Ont)

Microtus pennsylvanicus terraenovae (Bangs)

UNIRAMIA

SIPHONAPTERA

Megabothris a. asio (Nfld)

Microtus pinetorum scalopsoides (Audubon and

Bachman) Pine vole

Syn.: Pitymys pinetorum scalopsoides Batchelder

CHELICERATA

ACARI

Androlaelaps fahrenholzi(Ont)

UNIRAMIA

SIPHONAPTERA

Ctenophthalmus pseudagyrtes (Ont)

Hystrichopsylla tahavuana (Ont)

Microtus richardsoni (DeKay) Water vole UNIRAMIA

SIPHONAPTERA

Catallagia sculleni chamberlini (BC)

Megabothris abantis (Alta, BC, YT)

Microtus richardsoni richardsoni (DeKay)

UNIRAMIA

SIPHONAPTERA

Megabothris abantis (Alta, BC)

Microtus townsendii (Bachman) Townsend's vole

UNIRAMIA

DIPTERA

Cuterebra spp. (BC)

Wohlfahrtia vigil (BC)

SIPHONAPTERA

Delotelis hollandi (Alta, BC)

Hystrichopsylla o. occidentalis (BC)

Megabothris abantis (BC)

Opisodasys k. keeni (Alta, BC)

SIPHUNCULATA

Hoplopleura acanthopus (BC)

Microtus townsendii tetramerus (Rhoads)

Syn.: M. tetramerus

UNIRAMIA

SIPHONAPTERA

Atyphloceras m. multidentatus (BC)

Catallagia charlottensis (BC)

Nosopsyllus fasciatus (BC, Man, Nfld, NS,

Ont, Que) 
Microtus townsendii townsendii (Bachman)

UNIRAMIA

SIPHONAPTERA

Catallagia charlottensis $(\mathrm{BC})$

Corypsylla ornata $(\mathrm{BC})$

Delotelis hollandi $(\mathrm{BC})$

Epitedia wenmanni $(\mathrm{BC})$

Megabothris abantis (Alta, BC, YT)

Opisodasys k. keeni (Alta, BC)

Peromyscopsylla selenis (Alta, BC, Man,

NWT, Sask, YT)

Microtus xanthognathus (Leach) Yellow-cheeked vole

UNIRAMIA

SIPHONAPTERA

Amalaraeus dissimilis (Alta, BC, NWT, YT)

Amphipsylla sibirica pollionis (Alta, BC,

Nfld, YT)

Megabothris calcarifer gregsoni (NWT, YT)

M. groenlandicus (Man, Nfld, NWT, Que,

YT)

Peromyscopsylla h. hamifer (Alta, BC, Man, Nfld, NWT, Ont, Sask)

Microtus spp.

UNIRAMIA

SIPHONAPTERA

Amalaraeus athabascae (Alta, BC, Man,

Nfld, NWT, Que, Sask)

A. dissimilis (Alta, BC, NWT, YT)

Amphipsylla sibirica pollionis (Alta, BC, Nfld, YT)

Atyphloceras bishopi (Man, Nfld, Ont, Que)

Catallagia charlottensis (BC)

C. dacenkoi fulleri (Alta, BC, Man, NWT,

YT)

C. decipiens (Alta, BC, Man, Sask, YT)

C. sculleni chamberlini $(\mathrm{BC})$

Ceratophyllus lunatus tundrensis (Man, Nfld, NWT, Que, YT)

Corrodopsylla c. curvata (Alta, BC, Man,

NB, Nfld, NS, NWT, Ont, Que, Sask, YT)

Ctenophthalmus p. pseudagyrtes (Alta, BC,

Man, NB, NS, Ont, PEI, Que, Sask, YT)

Delotelis telegoni (Alta, BC)

Epitedia w. wenmanni (Alta, BC, Man, NB,

Nfld, NS, NWT, Ont, Que, Sask, YT)

Hystrichopsylla d. dippiei (Alta, BC, Man)

H. o. occidentalis (BC)

H. tahavuana (Que)

Malaraeus telchinus (Alta, BC)

Megabothris abantis (Alta, BC, YT)

M. a. asio (NB, Nfld, NS, Ont, PEI, Que)

M. a. megacolpus (Alta, BC, Man, NWT,

Ont, Sask)

M. calcarifer gregsoni (NWT, YT)

M. groenlandicus (Nfld, Que, YT)

M. lucifer (Alta, BC, Man, Sask)

M. quirini (Alta, BC, Man, NB, Nfld, NS,

NWT, Ont, Que, Sask, YT)

Monopsyllus thambus (Alta, BC, Nfld, NWT, Que, YT)
M. vison (Alta, BC, Man, Nfld, NS, NWT,

Ont, Que, Sask, YT)

$M$. wagneri ophidius $(\mathrm{BC})$

M. w. systaltus (Alta, Man, Ont, Sask)

M. w. wagneri (Alta, BC)

Nearctopsylla hyrtaci (Alta, BC)

Opisocrostis t. tuberculatus (Alta, BC, Sask,

YT)

Opisodasys k. keeni (Alta, BC)

Orchopeas leucopus (Alta, BC, Man, NB,

Nfld, NS, NWT, Ont, Que, Sask, YT)

Peromyscopsylla catatina (Man)

P. h. hamifer (Alta, BC, Man, Nfld, NWT,

Ont, Sask)

$P$. h. hesperomys (NS, Ont, Que)

P. ostsibirica longiloba (YT)

P. selenis (Alta, BC, Man, NWT, Sask, YT)

Rhadinopsylla linta (BC, NWT, YT)

Mus musculus L. House mouse

Syn.: Mus musculus musculus L.

CHELICERATA

ACARI

Myobiidae gen. spp. (Ont)

UNIRAMIA

DIPTERA

Cuterebra fontinella (Ont)

Cuterebra spp. (Ont)

SIPHONAPTERA

Epitedia w. wenmanni (Alta, BC, Man, NB,

NWT, Ont, Sask)

Leptopsylla segnis (BC, Nfld)

Malaraeus telchinus (Alta, BC)

Megabothris a. asio (NB, Nfld, NS, Ont, PEI,

Que)

Monopsyllus wagneri systaltus (Alta, Man,

Ont, Sask)

Myodopsylla palposa (BC)

Nosopsyllus fasciatus (BC)

Orchopeas leucopus (Alta, BC, Man, NB,

Nfld, NS, NWT, Ont, Que, Sask, YT)

Rhadinopsylla s. sectilis (BC)

SIPHUNCULATA

Hoplopleura hesperomydis (Ont)

Mus spp.

UNIRAMIA

SIPHONAPTERA

Malaraeus bitterootensis (Alta)

M. telchinus (Alta, BC)

Megarthroglossus d. divisus (Alta)

Peromyscopsylla h. hamifer (Alta, BC, Nfld,

Ont, Que)

Rhadinopsylla s. sectilis (BC)

Stenistomera alpina (Alta)

Stenopina americana (Alta, Man, Que)

Trichopsylla bitterootensis (Alta)

Neotoma cinerea (Ord) Bushy-tailed wood rat

CHELICERATA

ACARI

Ixodes ochotonae (BC)

UNIRAMIA 
SIPHONAPTERA

Anomiopsyllus montanus (Alta)

Catallagia charlottensis (Alta, BC)

C. decipiens (Alta, BC, Sask)

C. jellisoni (Alta, BC, YT)

Epitedia w. wenmanni (Alta, BC, Man, NB,

Nfld, NS, NWT, Ont, Que, Sask, YT)

Malaraeus bitterootensis (BC)

Megabothris abantis (Alta, BC, YT)

M. quirini (Alta, BC, Man, NB, Nfld, NS,

NWT, Ont, Que, Sask, YT)

Megarthroglossus d. divisus (Alta)

M. d. exsecatus $(\mathrm{BC})$

M. spenceri $(\mathrm{BC})$

Monopsyllus e. eumolpi (Alta, BC, Man,

NWT, Ont, Sask, YT)

Opisodasys k. keeni (Alta, BC)

Orchopeas sexdentatus agilis (Alta, BC, YT)

Peromyscopsylla ravalliensis (Alta, BC)

Rhadinopsylla arborea (Alta, BC)

Stenistomera alpina (Alta)

Neotoma cinerea cinerea (Ord)

CHELICERATA

ACARI

Dermacentor andersoni (Alta)

Ixodes spinipalpus (Alta)

UNIRAMIA

SIPHONAPTERA

Orchopeas sexdentatus agilis (Alta, BC, YT)

Neotoma cinerea drummondii (Richardson)

UNIRAMIA

SIPHONAPTERA

Megabothris abantis (Alta, BC, YT)

Megarthroglossus d. divisus (Alta, BC)

Monopsyllus $w$. wagneri (Alta, BC)

Orchopeas sexdentatus agilis (Alta, BC, YT)

Rhadinopsylla arborea (Alta, BC)

$R$. fraterna (Alta, BC, Sask)

Neotoma cinerea occidentalis Baird

Syn.: Neotoma cinerea saxamans Osgood

UNIRAMIA

SIPHONAPTERA

Amalaraeus dissimilis (Alta, BC, NWT, YT)

Megabothris abantis (Alta, BC)

Megarthroglossus sicamus (BC)

M. p. procus $(\mathrm{BC})$

Orchopeas sexdentatus agilis (Alta, BC, YT)

Peromyscopsylla ravalliensis (BC)

Rhadinopsylla linta (BC, NWT, YT)

SIPHUNCULATA

Hoplopleura acanthopus (BC)

Neohaematopinus inornatus (BC)

Neotoma spp.

UNIRAMIA

SIPHONAPTERA

Catallagia decipiens (Alta, BC, Man, Sask,

YT)

Epitedia w. wenmanni (Alta, BC, Man, NB,

Nfld, NS, NWT, Ont, Que, Sask, YT)
Malaraeus bitterootensis (Alta)

Megarthroglossus d. divisus (Alta)

M. sicamus (BC)

Monopsyllus e. eumolpi (Alta, BC, Man,

NWT, Ont, Sask, YT)

M. w. wagneri (Alta, BC)

Orchopeas sexdentatus agilis (Alta, BC, YT)

Peromyscopsylla ravallaensis (Alta, BC)

Rhadinopsylla difficilis $(\mathrm{BC})$

Ondatra zibethicus(L.) Muskrat CHELICERATA

Dermacarus ondatrae (Man, NS)

Dermacentor variabilis (NS)

Laelaps multispinosus (Man, NS, Ont)

Laelaps spp. (BC)

Listrophorus americanus (Man)

L. ondatrae (NWT)

L. validus (Ont)

Neotrombicula microti (NS)

Ondatra zibethicus osoyoosensis (Lord)

CHELICERATA

ACARI

Dermacerus validus (BC)

Eutrombicula harperi (BC)

Laelaps multispinosa (BC)

Listrophorus americanus (BC)

Radfordia spp. (BC)

Ondatra zibethicus zibethicus (L.)

CHELICERATA

ACARI

Dermacentor variabilis (NS)

Laelaps muris (Ont)

UNIRAMIA

SIPHONAPTERA

Orchopeas leucopus (Alta, BC, Man, NWT,

Ont, Que, Sask, YT)

Ondatra spp.

UNIRAMIA

SIPHONAPTERA

Amalaraeus dissimilis (Alta, BC, NWT, YT)

Onychomys leucogaster Baird

Northern

UNIRAMIA

SIPHONAPTERA

Epitedia w. wenmanni (Alta, BC, Man, NB,

Nfld, NS, NWT, Ont, Que, Sask, YT)

Onychomys leucogaster missouriensis (Audubon and

UNIRAMIA Bachman)

SIPHONAPTERA

Catallagia decipiens (Alta, BC, Sask)

Rhadinopsylla fraterna (Alta, BC, Sask)

Peromyscus leucopus (Rafinesque) White-footed

CHELICERATA

ACARI

Androlaelaps sp. (NS) 
Dermacentor variabilis (NS)

Eulaelaps stabularis (NS)

Haemogamasus sp. (NS)

Hypoaspis sp. (NS)

Ixodes angustus (NS)

Neotrombicula microti (NS)

Trombicula sp. (NS)

\section{UNIRAMIA}

DIPTERA

Cuterebra angustifrons (Ont)

SIPHONAPTERA

Catallagia charlottensis (Alta, BC)

Ctenophthalmus p. pseudagyrtes (NS)

Epitedia w. wenmanni (Alta, NS)

Megabothris quirini (Alta, BC, Man, NB,

Nfld, NS, NWT, Ont, Que, Sask, YT)

Monopsyllus vison (NS)

Orchopeas leucopus (Alta, BC, Man, NB,

Nfld, NS, NWT, Ont, Que, Sask)

Peromyscopsylla $h$. hesperomys (NS, Ont,

Que)

P. scotti(Ont)

Peromyscus leucopus caudatus Smith

CHELICERATA

ACARI

Ixodes muris (NS)

Peromyscus leucopus noveboracensis (Fischer)

UNIRAMIA

DIPTERA

Cuterebra angustifrons (Ont)

C. grisea (Ont)

Cuterebra spp. (Ont)

SIPHONAPTERA

Ctenophthalmus p. pseudagyrtes (Alta, Man, Ont, Sask)

Orchopeas leucopus (Alta, BC, Man, NWT,

Ont, Que, Sask, YT)

Peromyscus maniculatus (Wagner) Deer mouse

Syn.: Peromyscus canadensis of Rothschild (1906)

\section{CHELICERATA}

ACARI

Androlaelaps casalis (NB)

A. fahrenholzi (NB)

Androlaelaps sp. (NS)

Bakerdania spp. (NB)

Cyrtolaelaps spp. (NB)

Dermacarus jonesi (BC)

Dermacarus spp. (NB)

Dermacentor variabilis (Man, NS)

Eulaelaps stabularis (NB)

Euschoengastia setosa (NB)

Glycyphagidae gen. spp. (Ont)

Glycyphagus hypudaei (NB)

Haemolaelaps glasgowi(Ont)

Hirstionyssus spp. (NS, Ont)

Ixodes angustus (NB)

I. muris (PEI)

Ixodes spp. (Ont)

Labidophorids (Ont)

Miyatrombicula esoensis (NB)
Myobia spp. (Que)

Myobiidae gen. spp. (Ont)

Myocoptes spp. (Que)

Proctolaelaps spp. (NB)

Psorergates watsoni (Ont)

Radfordia subuliger (NB)

Radfordia spp. (Que)

Trichoecius spp. (NB)

Trombiculidae gen. spp. (Ont)

UNIRAMIA

DIPTERA

Cuterebra grisea $(\mathrm{BC})$

Cuterebra spp. (Ont)

SIPHONAPTERA

Amalaraeus athabascae (BC, Man, Nfld,

NWT, Que, Sask)

A. dissimilis (Alta, BC, NWT, YT)

Amphipsylla sibirica pollionis (Alta, BC,

Nfld, YT)

Atyphloceras m. multidentatus (BC)

Callistopsyllus t. terinus (BC)

Catallagia borealis (Man)

C. charlottensis $(\mathrm{BC})$

C. decipiens (Alta, BC, Man, Sask, YT)

C. jellisoni (Alta, BC, YT)

C. mathesoni $(\mathrm{BC})$

C. s. sculleni $(\mathrm{BC})$

Catallagia spp. (Man)

Corrodopsylla c. curvata (Alta, BC, Man,

NB, Nfld, NS, NWT, Ont, Que, Sask, YT)

Ctenophthalmus p. pseudagyrtes (NB, Ont,

Que)

Ctenopsyllus selenis $(\mathrm{BC})$

Delotelis hollandi $(\mathrm{BC})$

Epitedia scapani $(\mathrm{BC})$

E. w. wenmanni (Alta, BC, Man, NB, Nfld,

NS, NWT, Ont, Que, Sask, YT)

Hoplopleura hesperomydis (NB)

Hystrichopsylla d. dippiei (Alta, BC, Man,

Sask)

H. d. spinata $(\mathrm{BC})$

H. d. truncata $(\mathrm{BC})$

H. o. occidentalis (BC)

Malaraeus euphorbi (Alta, BC, NWT, Sask)

M. telchinus (Alta, BC)

Megabothris abantis (Alta, BC, YT)

M. asio (NB)

M. a. megacolpus (Alta, BC, Man, NWT,

Ont, Que, Sask, YT)

$M$. calcarifer gregsoni (NWT, YT)

M. quirini (Alta, BC, Man, NB, Nfld, NS,

NWT, Ont, Que, Sask, YT)

Megarthroglossus divisus exsecatus (BC)

M. p. procus $(\mathrm{BC})$

Monopsyllus ciliatus protinus $(\mathrm{BC})$

M. e. eumolpi (Alta, BC, Man, NWT, Ont,

Sask, YT)

M. thambus (Alta, BC, Nfld, NWT, Que,

Sask, YT)

M. vison (Alta, BC, Man, Nfld, NS, NWT,

Ont, Que, Sask, YT)

M. wagneri ophidius (BC) 
M. w. systaltus (Alta, Man, Ont, Sask)

M. w. wagneri (Alta, $\mathrm{BC}$ )

Nosopsyllus fasciatus (BC, Ont, Que, Sask)

Opisodasys k. keeni (Alta, BC)

Orchopeas c. caedens (Alta, BC, Man, Que,

YT)

O. c. durus (NS)

O. leucopus (Alta, BC, Man, NB, Nfld, NS, NWT, Ont, Que, Sask, YT)

Oropsylla arctomys (Alta, BC, Man, NB,

NWT, Ont, Que, Sask)

Peromyscopsylla catatina (NWT, Que)

P. h. hesperomys (NS)

$P$. h. hesperomys (NS)

$P$. h. pacifica $(\mathrm{BC})$

$P$. ravalliensis $(\mathrm{BC})$

$P$. selenis (Alta, BC, Man, NWT, Sask, YT)

Rhadinopsylla fraterna (BC, Sask)

R. linta (BC, NWT, YT)

$R$. sectilis goodi $(\mathrm{BC})$

R. s. sectilis (BC)

Stenistomera alpina (Alta)

SIPHUNCULATA

Hoplopleura hesperomydis (NB, NS, Ont, Que)

Peromyscus maniculatus abietorum Bangs

CHELICERATA

ACARI

Dermacentor variabilis (NS)

UNIRAMIA

SIPHONAPTERA

Catallagia borealis (NB)

Ctenophthalmus p. pseudagyrtes (NB)

Epitedia w. wenmanni (NB)

Malaraeus euphorbi (Alta, BC, Sask)

M. telchinus (Alta, BC)

Orchopeas caedens durus (NB)

O. leucopus (NB)

Stenoponia americana (NB)

Peromyscus maniculatus algidus Osgood

UNIRAMIA

SIPHONAPTERA

Epitedia w. wenmanni (Alta, BC, Man, NB,

Nfld, NS, NWT, Ont, Que, Sask, YT)

Megabothris quirini (Alta, BC, Man, NB,

Nfld, NS, NWT, Ont, Que, Sask, YT)

Monopsyllus thambus (Alta, BC, Nfld, NWT,

Que, YT)

Orchopeas leucopus (Alta, BC, Man, NB,

Nfld, NS, NWT, Ont, Que, Sask)

Peromyscus maniculatus alpinus Cowan

UNIRAMIA

SIPHONAPTERA

Catallagia decipiens (Alta, BC, Man, Sask,

YT)

Hystrichopsylla o. occidentalis (Alta, BC)

Megabothris abantis (Alta, BC, YT)

Monopsyllus w. wagneri (Alta, BC)

Opisodasys k. keeni (Alta, BC)
Peromyscus maniculatus anticostiensis Moulthrop

UNIRAMIA

SIPHONAPTERA

Ctenophthalmus p. pseudagyrtes (Alta, BC, Man, NB, NS, Ont, PEI, Que, Sask)

Peromyscus maniculatus artemisiae (Rhoads)

UNIRAMIA

SIPHONAPTERA

Callistopsyllus t. terinus (BC)

Catallagia decipiens (Alta, BC, Man, Sask,

YT)

Epitedia w. wenmanni (Alta, BC, Man, NB,

Nfld, NS, NWT, Ont, Que, Sask, YT)

Hystrichopsylla dippiei truncata $(\mathrm{BC})$

Megabothris abantis (Alta, BC)

M. quirini (Alta, BC, NWT, Ont)

Meringis shannoni $(\mathrm{BC})$

Monopsyllus e. eumolpi (Alta, BC, Man,

NWT, Ont, Sask, YT)

M. w. wagneri (Alta, BC)

Opisodasys k. keeni (Alta, BC)

Rhadinopsylla s. sectilis (BC)

SIPHUNCULATA

Hoplopleura hesperomydis (BC)

Peromyscus maniculatus austerus (Baird)

CHELICERATA

ACARI

Radfordia subuliger (BC)

Trombicula spp. (BC)

UNIRAMIA

DIPTERA

Cuterebra grisea $(\mathrm{BC})$

Cuterebra spp. (BC)

SIPHONAPTERA

Atyphloceras m. multidentatus (BC)

Catallagia charlottensis (BC)

C. s. sculleni $(\mathrm{BC})$

Delotelis hollandi (Alta, BC)

Epitedia scapani (BC)

Hystrichopsylla o. occidentalis (BC)

Malaraeus telchinus (Alta, BC)

Megabothris abantis (Alta, BC)

Monopsyllus cileatus protinus (BC)

$M$. wagneri ophidius (BC)

Opisodasys k. keeni (Alta, BC)

Peromyscopsylla hesperomys pacifica $(\mathrm{BC})$

Rhadinopsylla sectilis goodi $(\mathrm{BC})$

SIPHUNCULATA

Hoplopleura hesperomydis (BC)

Peromyscus maniculatus bairdii (Hoy and Kennicott) UNIRAMIA

SIPHONAPTERA

Catallagia borealis (Man, NB, Ont, Que)

Ctenophthalmus p. pseudagyrtes (Alta, BC,

Man, NB, NS, Ont, PEI, Que, Sask)

Epitedia w. wenmanni (Alta, BC, Man, NB,

Nfld, NS, NWT, Ont, Que, Sask, YT)

Foxella ignota albertensis (Man)

Hystrichopsylla d. dippiei (Alta, BC, Man) 
Megabothris asio megacolpus (Alta, BC,

Man, NWT, Ont, Que, Sask, YT)

M. quirini (Alta, BC, Man, NB, Nfld, NS,

NWT, Ont, Que, Sask, YT)

Monopsyllus wagneri systaltus (Alta, Man,

Ont, Sask)

Orchopeas leucopus (Alta, BC, Man, NB,

Nfld, NS, NWT, Ont, Que, Sask, YT)

Peromyscopsylla catatina (Man, Nfld, NS,

Ont)

Peromyscus maniculatus borealis Mearns

Syn.: Peromyscus arcticus of Rothschild, 1905

UNIRAMIA

SIPHONAPTERA

Amalaraeus dissimilis (BC)

Catallagia charlottensis (Alta, BC)

C. decipiens (Alta, BC, Man, Sask, YT)

Ctenophthalmus p. pseudagyrtes (Alta, Man,

Ont, Sask)

Epitedia w. wenmanni (Alta, BC, Man, NB,

Nfld, NS, NWT, Ont, Que, Sask, YT)

Malaraeus telchinus (BC)

Megabothris abantis (Alta, BC)

M. asio megacolpus (Man)

M. quirini (Alta, BC, Man, NB, Nfld, NS,

NWT, Ont, Que, Sask, YT)

Monopsyllus thambus (Alta, BC, Nfld, NWT,

Que, YT)

M. w. wagneri (Alta, BC)

Opisodasys k. keeni (Alta, BC)

Orchopeas leucopus (Alta, BC, Man, NB,

Nfld, NS, NWT, Ont, Que, Sask, YT)

Peromyscopsylla selenis (BC, Man, NWT,

Sask, YT)

SIPHUNCULATA

Polyplax auricularis (Alta)

Peromyscus maniculatus gracilis (Le Conte)

UNIRAMIA

DIPTERA

Cuterebra spp. (Ont)

SIPHONAPTERA

Atyphloceras bishopi (Que)

Epitedia w. wenmanni (Alta, BC, Man, NB,

NWT, Ont, Que, Sask)

Orchopeas leucopus (Alta, BC, Man, NWT,

Ont, Que, Sask, YT)

Stenoponia americana (Man, Ont, Que)

Peromyscus maniculatus interdictus Anderson

UNIRAMIA

SIPHONAPTERA

Catallagia charlottensis (BC)

Malaraeus telchinus (BC)

Opisodasys k. keeni (Alta, BC)

Peromyscopsylla hesperomys pacifica $(\mathrm{BC})$

Peromyscus maniculatus keeni (Rhoads)

UNIRAMIA

SIPHONAPTERA

Catallagia charlottensis (BC)

Opisodasys k. keeni (Alta,BC)
Peromyscus maniculatus macrorhinus (Rhoads)

UNIRAMIA

SIPHONAPTERA

Catallagia charlottensis (BC)

Peromyscus maniculatus maniculatus (Wagner)

UNIRAMIA

DIPTERA

Cuterebra spp. (Ont)

SIPHONAPTERA

Monopsyllus w. wagneri (Alta, BC)

Peromyscus maniculatus nebrascensis (Coues)

Syn.: Peromyscus maniculatus osgoodi Mearns

UNIRAMIA

SIPHONAPTERA

Callistopsyllus terinus campestris (Alta, Sask)

Catallagia decipiens (Alta, BC, Sask)

Epitedia w. wenmanni (Alta, BC, Man, NB,

NWT, Ont, Sask)

Hystrichopsylla d. dippiei (Alta, Sask)

Malaraeus euphorbi (Alta, BC, Sask)

Megabothris lucifer (Alta, BC, Sask)

Monopsyllus wagneri systaltus (Alta, Man,

Ont, Sask)

Neopsylla inopina (Alta, BC, Sask)

Orchopeas leucopus (Alta, BC, Man, NWT,

Ont, Que, Sask, YT)

Rhadinopsylla fraterna (Alta, BC, Sask)

$R$. rauschi (Sask)

Peromyscus maniculatus oreas Bangs

UNIRAMIA

SIPHONAPTERA

Catallagia charlottensis (BC)

Delotelis hollandi (Alta, BC)

Epitedia scapanus (BC)

Malaraeus telchinus (BC)

Megabothris abantis (Alta, BC)

Monopsyllus ciliatus protinus (BC)

$M$. wagneri ophidius $(\mathrm{BC})$

Opisodasys k. keeni (Alta, BC)

Peromyscopsylla hesperomys pacifica $(\mathrm{BC})$

Rhadinopsylla sectilis goodi (BC)

SIPHUNCULATA

Hoplopleura hesperomydis (BC)

Peromyscus maniculatus prevostensis Osgood

Syn.: Peromyscus sitkensis prevostensis of Spencer (1966)

UNIRAMIA

SIPHUNCULATA

Hoplopleura hesperomydis (BC)

Peromyscus sitkensis Merriam

UNIRAMIA

SIPHONAPTERA

Malaraeus telchinus (Alta, BC)

Opisodasys k. keeni (Alta, BC)

Peromyscus spp.

CHELICERATA

ACARI

Androlaelaps fahrenholzi (Ont)
Sitka mouse 
Dermacentor variabilis (NS)

Eulaelaps stabularis (Ont)

Ixodes angustus (Ont)

I. californicus $(\mathrm{BC})$

I. ochotonae (BC)

I. pacificus $(\mathrm{BC})$

Myobia spp. (BC)

UNIRAMIA

\section{SIPHONAPTERA}

Amalaraeus dissimilis (Alta, BC, NWT, YT)

Atyphloceras m. multidentatus (BC)

Callistopsyllus terinus campestris (Alta)

C. t. terinus $(\mathrm{BC})$

Catallagia charlottensis (BC)

C. dacenkoi fulleri (Alta, BC, Man, YT)

C. decipiens (Alta, BC, Man, Sask, YT)

C. sculleni chamberlini $(\mathrm{BC})$

C. s. sculleni $(\mathrm{BC})$

Ceratophyllus garei (Alta, BC, Man, NWT,

Que, YT)

Ctenophthalmus p. pseudagyrtes (Alta, BC, Man, NB, NS, Ont, PEI, Que, Sask)

Dasypsyllus gallinulae perpinnatus $(\mathrm{BC})$

Delotelus hollandi (Alta, BC)

Doratopsylla blarinae (NB, NS, Ont, Que)

Epitedia scapani $(\mathrm{BC})$

E. stewarti (BC)

Megabothris asio megacolpus (Alta, BC,

Man, NWT, Ont, Que, Sask, YT)

M. quirini (Alta, BC, Man, NB, Nfld, NS,

NWT, Ont, Que, Sask, YT)

Monopsyllus wagneri systaltus (Alta, Man,

Ont, Sask)

Orchopeas leucopus (Alta, BC, Man, NB,

Nfld, NS, NWT, Ont, Que, Sask, YT)

Peromyscopsylla catatina (Man, Nfld, NS,

Ont)

Peromyscus spp.

CHELICERATA

ACARI

Androlaelaps fahrenholzi(Ont)

Dermacentor variabilis (NS)

Eulaelaps stabularis (Ont)

Ixodes angustus (Ont)

I. californicus $(\mathrm{BC})$

I. ochotonae (BC)

I. pacificus $(\mathrm{BC})$

Myobia spp. (BC)

UNIRAMIA

SIPHONAPTERA

Amalaraeus dissimilis (Alta, BC, NWT, YT)

Atyphloceras m. multidentatus (BC)

Callistopsyllus terinus campestris (Alta)

C. t. terinus (BC)

Catallagia charlottensis (BC)

C. dacenkoi fulleri (Alta, BC, Man, YT)

C. decipiens (Alta, BC, Man, Sask, YT)

C. sculleni chamberlini $(\mathrm{BC})$

C. s. sculleni (BC)

Ceratophyllus garei (Alta, BC, Man, NWT, Que, YT)
Ctenophthalmus p. pseudagyrtes (Alta, BC,

Man, NB, NS, Ont, PEI, Que, Sask)

Dasypsyllus gallinulae perpinnatus $(\mathrm{BC})$

Delotelus hollandi (Alta, BC)

Doratopsylla blarinae (NB, NS, Ont, Que)

Epitedia scapani $(\mathrm{BC})$

E. stewarti (BC)

E. w. wenmanni (Alta, BC, Man, NB, Nfld, NS, NWT, Ont, Que, Sask, YT)

Hystrichopsylla d. dippiei (BC)

H. d. truncata $(\mathrm{BC})$

H. o. occidentalis (Alta, BC)

Malaraeus abantis (Alta, BC, YT)

M. euphorbi (Alta, BC, NWT, Sask)

M. telchinus (Alta, BC)

Megabothris abantis (Alta, BC, YT)

M. a. asio (NB, Nfld, NS, Ont, PEI, Que)

M. a. megacolpus (Alta, BC, Man, NWT,

Ont, Que, Sask, YT)

M. groenlandicus (Man, Nfld, NWT, Que,

YT)

M. lucifer (Alta, BC, Man, Sask)

M. quirini (Alta, BC, Man, NB, Nfld, NS,

NWT, Ont, Que, Sask, YT)

Megarthroglossus p. procus (BC)

Monopsyllus ciliatus protinus (BC)

M. vison (Alta, BC, Man, Nfld, NS, NWT,

Ont, Que, Sask, YT)

$M$. wagneri ophidius (BC)

M. w. systaltus (Alta, Man, Ont, Sask)

M. w. wagneri (Alta, BC)

Opisodasys $k$. keeni (Alta, BC)

Orchopeas leucopus (Alta, BC, Man, NB,

Nfld, NS, NWT, Ont, Que, Sask, YT)

O. sexdentatus agilis (Alta, BC, YT)

Oropsylla rupestris (Alta, Man, Sask, YT)

Peromyscopsylla hesperomys adelpha (Alta, Sask)

P. h. hesperomys (NS, Ont, Que)

P. h. pacifica $(\mathrm{BC})$

P. selenis (Alta, BC, Man, NWT, Sask, YT)

Rhadinopsylla difficilis (BC)

$R$. sectilis goodi $(\mathrm{BC})$

R. s. sectilis ( $\mathrm{BC})$

Thrassis acamantis (Alta, BC)

Trichopsylla wagneri systalta (Alta)

SIPHUNCULATA

Hoplopleura hesperomydis (BC)

Phenacomys intermedius Merriam Heather vole CHELICERATA

ACARI

Listrophorus phenacomys (Man, NWT)

UNIRAMIA

SIPHONAPTERA

Amalaraeus dissimilis (Alta, BC, NWT, YT)

Catallagia decipiens (Alta, BC, Man, Sask, YT)

Hystrichopsylla o. occidentalis (BC)

Malaraeus telchinus (Alta, BC)

Megabothris abantis (Alta, BC, YT)

Peromyscopsylla selenis (Alta, BC, Man,

NWT, Sask, YT) 
Rhadinopsylla fraterna (Alta, BC, Sask)

Phenacomys intermedius celatus Merriam

Syn.: Phenacomys ungava Merriam

Phenacomys ungava ungava Merriam

UNIRAMIA

SIPHONAPTERA

Epitedia w. wenmanni (Alta, BC, Man, NB,

Nfld, NS, NWT, Ont, Que, Sask, YT)

Megabothris asio (Nfld)

M. a. asio (NB, Nfld, NS, Ont, PEI, Que)

M. quirini (Alta, BC, Man, NB, Nfld, NS, NWT, Ont, Que, Sask, YT)

Phenacomys intermedius intermedius Merriam

UNIRAMIA

SIPHONAPTERA

Megabothris abantis (Alta, BC)

Phenacomys spp.

CHELICERATA

ACARI

Listrophorus phenacomys (Man, NWT)

UNIRAMIA

SIPHONAPTERA

Amalaraeus dissimilis (Alta, BC, NWT, YT)

Epitedia w. wenmanni (Alta, BC, Man, NB,

Nfld, NS, NWT, Ont, Que, Sask, YT)

Megabothris a. asio (NB, Nfld, NS, Ont, PEI,

Que)

M. a. megacolpus (Alta, BC, Man, NWT,

Ont, Que, Sask, YT)

M. quirini (Alta, BC, Man, NB, Nfld, NS,

NWT, Ont, Que, Sask, YT)

Rhadinopsylla difficilis (BC)

Rattus norvegicus (Berkenhout) Norway rat

Syn.: Rattus rattus norvegicus of Spencer (1939)

CHELICERATA

ACARI

Androlaelaps fahrenholzi(Ont)

Echinolaelaps echidninus (Que)

Laelaps spp. (Que)

Ornithonyssus bacoti (BC)

Radfordia spp. (Que)

UNIRAMIA

DIPTERA

Cuterebra spp. (Sask)

SIPHONAPTERA

Atyphloceras m. multidentatus (BC)

Catallagia charlottensis (BC)

Ctenocephalides canis (BC)

C. felis (BC)

C. f. felis (BC)

Ctenophthalmus p. pseudagyrtes (NB, Que)

Epitedia scapani (BC)

Hystrichopsylla o. occidentalis (BC)

Megabothris abantis (Alta, BC, YT)

M. quirini (Que)

Megarthroglossus p. procus (BC)

Neopsylla inopina (Alta, BC, Sask)

Nosopsyllus fasciatus (BC, Man, Nfld, NS,

Ont, Que)
Oropsylla rupestris (Alta, Man, Sask, YT)

Peromyscopsylla selenis (Alta, BC, Man,

NWT, Sask)

Rhadinopsylla sectilis goodi $(\mathrm{BC})$

Siphonaptera gen. spp. (BC)

Xenopsylla cheopis (BC, NB, NS, Ont, Que)

SIPHUNCULATA

Haematopinus spinulosus (Que)

Hoplopleura acanthopus (BC)

H. oenomydis $(\mathrm{BC})$

Polyplax spinulosa (BC, Que)

Rattus rattus (L.) Black rat

CHELICERATA

ACARI

Ornithonyssus bacoti (BC)

UNIRAMIA

SIPHONAPTERA

Hystrichopsylla o. occidentalis (BC)

Rattus rattus alexandrinus (Desmarest)

UNIRAMIA

SIPHONAPTERA

Nosopsyllus fasciatus (BC)

Siphonaptera gen. spp. (BC)

Xenopsylla cheopis (BC)

Rattus rattus rattus (L.)

UNIRAMIA

SIPHONAPTERA

Nosopsyllus fasciatus (BC)

Xenopsylla cheopis (BC)

SIPHUNCULATA

Polyplax spinulosa (BC)

Rattus spp.

UNIRAMIA

DIPTERA

Cuterebra sp. (Sask)

SIPHONAPTERA

Megabothris a. asio (NB, Nfld, NS, Ont, PEI,

Que)

Nosopsyllus fasciatus (BC, Man, Nfld, NS,

Ont, Que, Sask)

Orchopeas leucopus (Alta, BC, Man, NB,

Nfld, NS, NWT, Ont, Que, Sask)

SIPHUNCULATA

Polyplax spinulosa $(\mathrm{BC})$

Synaptomys borealis (Richardson) Northern bog lemming

UNIRAMIA

SIPHONAPTERA

Amalaraeus athabascae (Alta, BC, Man,

Nfld, NWT, Que, Sask)

A. dissimilis (Alta, BC, NWT, YT)

Ctenophthalmus p. pseudagyrtes (NB)

Epitedia w. wenmanni (NB)

Megabothris asio megacolpus (Alta, BC,

Man, NWT, Ont, Que, Sask, YT)

$M$. calcarifer gregsoni (NWT, YT)

M. quirini (Alta, BC, Man, NB, Nfld, NS,

NWT, Ont, Que, Sask, YT) 
Monopsyllus w. wagneri (Alta, BC)

Synaptomys borealis borealis (Richardson)

UNIRAMIA

SIPHONAPTERA

Peromyscopsylla selenis (Alta, BC, Man, NWT, Sask)

Synaptomys borealis chapmani Allen

CHELICERATA

ACARI

Androlaelaps fahrenholzi (NB)

Euryparasitus spp. (NB)

Glycyphagus hypudaei (NB)

Ixodes angustus (NB)

Laelaps alaskensis (NB)

Listrophorus synaptomys (NB)

Miyatrombicula esoensis (NB)

Orycteroxenus soricis (NB)

Pygmephorus horridus (NB)

Radfordia hylandi (NB)

UNIRAMIA

SIPHONAPTERA

Ctenophthalmus pseudagyrtes (NB)

Epitedia w. wenmanni(NB)

Megabothris quirini (NB)

SIPHUNCULATA

Hoplopleura acanthopus (Alta,BC)

Synaptomys borealis medioximus Bangs

UNIRAMIA

SIPHONAPTERA

Megabothris a. asio (Nfld)

Synaptomys borealis truei Merriam

Syn.: Synaptomys borealis wrangeli Merriam

UNIRAMIA

SIPHONAPTERA

Megabothris abantis (Alta,BC)

Synaptomys cooperi Baird

Southern bog lemming

\section{CHELICERATA}

ACARI

Androlaelaps fahrenholzi (NB)

Bakerdania spp. (NB)

Dermacentor variabilis (NS)

Echinonyssus isabellinus (NB)

Eulaelaps stabularis (NB)

Euryparasitus spp. (NB)

Glycyphagus hypudaei (NB)

Haemogamasus ambulans (NB)

H. liponyssoides (NB)

Ixodes angustus (NB)

I. muris (NS)

Laelaps alaskensis (NB)

L. kochi (NB)

Listrophorus mexicanus (NB)

L. synaptomys (NB)

Megabothris quirini (NB)

Miyatrombicula esoensis (NB)

Neotrombicula harperi (NB)

Pygmephorus horridus (NB)
Radfordia hylandi (NB)

\section{UNIRAMIA}

SIPHONAPTERA

Ctenophthalmus p. pseudagyrtes (NB)

Epitedia w. wenmanni (NB)

Megabothris a. asio (NB, Nfld, NS, Ont, PEI,

Que)

M. quirini (NB)

SIPHUNCULATA

Hoplopleura acanthopus (NB)

Synaptomys cooperi cooperi Baird

CHELICERATA

ACARI

Ixodes muris (NS)

Synaptomys spp.

UNIRAMIA

SIPHONAPTERA

Amphipsylla sibirica pollionis (Alta, BC,

Nfld, YT)

Catallagia charlottensis $(\mathrm{BC})$

Megabothris abantis (Alta, BC, YT)

M. a. asio (NB, Nfld, NS, Ont, PEI, Que)

M. a. megacolpus (Alta, BC, Man, NWT,

Ont, Que, Sask, YT)

M. groenlandicus (Man, Nfld, NWT, Que, YT)

M. lucifer (Alta, BC, Man, Sask)

Monopsyllus w. wagneri (Alta, BC)

Peromyscopsylla catatina (NB)

P. selenis (Alta, BC, Man, NWT, Sask, YT)

Thrassis acamantis (Alta, BC)

\section{MURIDAE OF UNDETERMINED SPECIES}

Deer mouse

UNIRAMIA

SIPHONAPTERA

Malaraeus euphorbi (Alta, BC, NWT, Sask)

Field mouse

CHELICERATA

ACARI

Dermacentor andersoni $(\mathrm{BC})$

Psorergates spp. (Ont)

UNIRAMIA

DIPTERA

Cuterebra grisea (Sask)

SIPHONAPTERA

Monopsyllus ciliatus protinus (BC)

House mouse

CHELICERATA

ACARI

Myobia musculi(Ont)

Psorergates sp. (Ont)

UNIRAMIA

DIPTERA

Cuterebra grisea $(\mathrm{BC})$

Cuterebra sp. (Man)

SIPHONAPTERA

Monopsyllus thambus (Nfld)

$M$. wagneri systaltus (Alta, Man, Ont, Sask) 
Kangaroo mouse

UNIRAMIA

SIPHONAPTERA

Ctenopsyllus selenis (Alta)

Lab rat

UNIRAMIA

\section{DIPTERA}

Wohlfahrtia meigenii (Alta)

Lemming

UNIRAMIA

SIPHONAPTERA

Ceratophyllus garei (Alta, BC, Man, NWT,

Que, YT)

Megabothris a. asio (NB, Nfld, NS, Ont, PEI,

Que)

M. groenlandicus (Man)

Meadow vole

UNIRAMIA

SIPHONAPTERA

Megabothris $a$. asio (NB, Nfld, NS, Ont, PEI, Que)

Mice

CHELICERATA

ACARI

Dermacentor andersoni (Alta, BC, Ont)

Ixodes angustus $(\mathrm{BC})$

I. cookei $(\mathrm{BC})$

I. pacificus $(\mathrm{BC})$

Psorergates simplex (unspecified)

UNIRAMIA

DIPTERA

Cuterebra grisea (Alta)

SIPHONAPTERA

Amalaraeus dissimilis (Alta, BC, NWT, YT)

Ctenophthalmus p. pseudagyrtes (NB, NS,

Ont)

Malaraeus telchinus (Alta, BC)

Megabothris abantis (Alta, BC)

M. groenlandicus (Man)

Monopsyllus thambus (Alta, BC, Nfld, NWT,

Que, YT)

M. w. wagneri (Alta, BC)

Nosopsyllus fasciatus (BC, Ont, Que, Sask)

Orchopeas leucopus (Alta, BC, Man, NWT,

Ont, Que, Sask, YT)

Packrat

CHELICERATA

ACARI

Dermacentor andersoni $(\mathrm{BC})$

Ixodes cookei (BC)

Pallid vole

UNIRAMIA

SIPHONAPTERA

Amphipsylla washingtona (Alta)

Pocket gopher

UNIRAMIA
SIPHONAPTERA

Foxella ignota albertensis (Alta, Sask)

F. i. ignota (Man)

F. i. recula $(\mathrm{BC})$

Pocket mouse

CHELICERATA

ACARI

Dermacentor andersoni (BC)

Red-backed mouse

UNIRAMIA

SIPHONAPTERA

Ceratophyllus lunatus tundrensis (Man, Nfld, NWT, Que, YT)

Red-backed vole

UNIRAMIA

SIPHONAPTERA

Malaraeus telchinus (Alta, BC)

Vole

CHELICERATA

ACARI

Ixodes angustus $(\mathrm{BC})$

UNIRAMIA

DIPTERA

Cuterebra sp. (unspecified)

SIPHONAPTERA

Catallagia decipiens (Alta, BC, Man, Sask, YT)

White mouse

CHELICERATA

ACARI

Dermacentor andersoni $(\mathrm{BC})$

Wood rat

UNIRAMIA

SIPHONAPTERA

Orchopeas sexdentatus agilis (Alta, BC, YT)

Family SCIURIDAE

Cynomys ludovicianus Baird

Black-tailed

UNIRAMIA prairie dog

SIPHONAPTERA

Opisocrostis labis (Alta, Sask)

Thrassis b. bacchi (Alta, Man, Sask)

Cynomys ludovicianus ludovicianus (Ord)

UNIRAMIA

SIPHONAPTERA

Opisocrostis labis (Alta, Sask)

O. t. tuberculatus (Alta, BC, Sask)

Oropsylla rupestris (Alta, Man, Sask)

Thrassis b. bacchi (Alta, Man, Sask)

SIPHUNCULATA

Neohaematopinus marmotae (Sask)

Eutamias amoenus Allen

Yellow-pine

UNIRAMIA chipmunk 
Monopsyllus ciliatus protinus (BC)

M. eumolpi (Alta, BC, Man, NWT, Sask,

Ont, YT)

Orchopeas caedens durus (BC)

Eutamias amoenus affinis (Allen)

UNIRAMIA

SIPHONAPTERA

Catallagia decipiens (Alta, BC, Man, Sask,

YT)

Hystrichopsylla d. dippiei (BC)

Monopsyllus eumolpi (Alta, BC, Ont, Sask)

M. e. eumolpi (Alta, BC, Man, NWT, Ont,

Sask, YT)

SIPHUNCULATA

Hoplopleura arboricola $(\mathrm{BC})$

Neohaematopinus sciuropteri (BC)

Eutamias amoenus felix (Rhoads)

UNIRAMIA

SIPHONAPTERA

Monopsyllus ciliatus protinus (BC)

Eutamias amoenus ludibondus Hollister

UNIRAMIA

SIPHONAPTERA

Monopsyllus eumolpi (Alta,BC,Ont,Sask)

Eutamias amoenus luteiventris (Allen)

UNIRAMIA

SIPHONAPTERA

Monopsyllus eumolpi (Alta, BC, Ont, Sask)

M. e. eumolpi (Alta, BC, Man, NWT, Ont,

Sask, YT)

SIPHUNCULATA

Hoplopleura arboricola $(\mathrm{BC})$

Eutamias minimus (Bachman) Least chipmunk CHELICERATA

ACARI

Haemogamasus ambulans (Ont)

UNIRAMIA

SIPHONAPTERA

Amalaraeus dissimilis (Alta, BC, NWT, YT)

Ctenophthalmus p. pseudagyrtes (Alta, BC,

Man, NB, NS, Ont, PEI, Que, Sask)

Megabothris acerbus (Man, Ont)

M. quirini (Alta, BC, Man, NB, Nfld, NS,

NWT, Ont, Que, Sask, YT)

Monopsyllus e. eumolpi (Alta, BC, Man,

NWT, Ont, Sask, YT)

Orchopeas sexdentatus agilis (Alta, BC, YT)

Eutamias minimus borealis (Allen)

Syn.: Eutamias borealis of Holland (1950)

UNIRAMIA

SIPHONAPTERA

Ctenophthalmus p. pseudagyrtes (Alta, BC,

Man, NB, NS, Ont, PEI, Que, Sask)

Megabothris asio megacolpus (Man)

M. quirini (Alta, BC, Man, NWT, Ont)

Monopsyllus eumolpi (Alta, BC, Ont, Sask)

M. e. eumolpi (Alta, BC, Man, NWT, Ont,
Sask, YT)

M. tolli (YT)

M. vison (Alta, BC, Man, Nfld, NS, NWT,

Ont, Que, Sask, YT)

Opisocrostis bruneri (unspecified)

Eutamias minimus minimus (Bachman)

UNIRAMIA

SIPHONAPTERA

Monopsyllus e. eumolpi (Alta, BC, Man,

NWT, Ont, Sask, YT)

Eutamias minimus selkirki Cowan

UNIRAMIA

SIPHONAPTERA

Monopsyllus eumolpi (Alta, BC, Ont, Sask)

Eutamias townsendii (Bachman)

Townsend's

UNIRAMIA chipmunk

SIPHONAPTERA

Monopsyllus ciliatus protinus (BC)

Oropsylla idahoensis (Alta, BC, YT)

Eutamias townsendii cooperi (Baird)

UNIRAMIA

SIPHONAPTERA

Monopsyllus ciliatus protinus $(\mathrm{BC})$

Eutamias spp.

CHELICERATA

ACARI

Ixodes pacificus $(\mathrm{BC})$

UNIRAMIA

SIPHONAPTERA

Catallagia charlottensis (BC)

C. decipiens (Alta, BC, Man, Sask, YT)

Ceratophyllus diffinis $(\mathrm{BC})$

Ctenophthalmus p. pseudagyrtes (Alta, BC,

Man, NB, NS, Ont, PEI, Que, Sask)

Epitedia w. wenmanni (Alta, BC, Man, NB,

NWT, Ont, Sask)

Megabothris asinus (Alta, BC)

M. asio megacolpus (Alta, BC, Man, NWT,

Ont, Que, Sask, YT)

M. lucifer (Alta, BC, Man, Sask)

M. quirini (Alta, BC, Man, NB, Nfld, NS,

NWT, Ont, Que, Sask, YT)

Monopsyllus eumolpi (Alta, BC, Ont, Sask)

M. e. eumolpi (Alta, BC, Man, NWT, Ont,

Sask, YT)

M. vison (Alta)

M. w. wagneri (Alta, BC)

Opisodasys k. keeni (Alta, BC)

Orchopeas c. caedens (Alta, BC, Man, Que,

YT)

O. c. durus (BC, Nfld, NWT)

Oropsylla idahoensis (Alta, BC, YT)

Thrassis acamantis (Alta)

$T$. petiolatus (Alta, BC)

Trichopsylla e. eumolpi (Alta)

T. vison (Alta) 
CHELICERATA

ACARI

Haemogamasus sp. (NS)

Ixodes marxi (NS)

Laelaps kochi (Ont)

UNIRAMIA

SIPHONAPTERA

Conorhinopsylla stanfordi(Ont)

Epitedia faceta (Ont, Que)

E. w. wenmanni (NB)

Megarthroglossus divisus exsecatus (BC)

Monopsyllus ciliatus protinus (BC)

M. vison (Alta, BC, Man, Nfld, NS, NWT,

Ont, Que, Sask, YT)

Opisodasys pseudarctomys (Alta, BC, Man,

NS, Ont, Que)

O. vesperalis $(\mathrm{BC})$

Orchopeas c. caedens (Alta, BC, Man, Que,

YT)

O. c. durus (Alta, BC, NB, Nfld, NS, NWT, Ont, Que, Sask)

Tarsopsylla octodecimdentata coloradensis

(Alta, BC, Man, NWT, Ont, Sask, YT)

SIPHUNCULATA

Neohaematopinus sciuropteri (Alta)

Glaucomys sabrinus alpinus (Richardson)

UNIRAMIA

SIPHONAPTERA

Opisodasys pseudarctomys (BC)

$O$. vesperalis $(\mathrm{BC})$

Tarsopsylla octodecemdentata coloradensis (BC)

SIPHUNCULATA

Neohaematopinus sciuropteri (BC)

Glaucomys sabrinus canescens Howell

UNIRAMIA

SIPHONAPTERA

Opisodasys pseudarctomys (Alta, BC, Man, NS, Ont, Que)

Glaucomys sabrinus columbiensis Howell

UNIRAMIA

SIPHONAPTERA

Opisodasys pseudarctomys (Alta, BC, Man,

NS, Ont, Que)

$O$. vesperalis $(\mathrm{BC})$

SIPHUNCULATA

Hoplopleura trispinosa (BC)

Neohaematopinus sciuropteri $(\mathrm{BC})$

Glaucomys sabrinus fuliginosus (Rhoads)

UNIRAMIA

SIPHONAPTERA

Megarthroglossus divisus exsecatus (BC)

Opisodasys vesperalis (BC)

Glaucomys sabrinus gouldi Anderson

CHELICERATA

ACARI
Ixodes marxi (NS)

Glaucomys sabrinus macrotis (Mearns)

UNIRAMIA

SIPHONAPTERA

Epitedia w. wenmanni (NB)

Opisodasys pseudarctomys (Alta, NB, Ont)

Orchopeas leucopus (Alta, BC, Man, NWT,

Ont, Que, Sask, YT)

Glaucomys sabrinus makkovikensis (Sornborger)

UNIRAMIA

SIPHONAPTERA

Opisodasys pseudarctomys (Nfld)

Glaucomys sabrinus oregonensis (Bachman)

CHELICERATA

ACARI

Acari gen. spp. (BC)

Ixodes pacificus $(\mathrm{BC})$

UNIRAMIA

SIPHONAPTERA

Monopsyllus ciliatus protinus (BC)

Opisodasys vesperalis (BC)

Orchopeas nepos (BC)

SIPHUNCULATA

Hoplopleura trispinosa (Alta, BC)

Microphthirus uncinatus (Alta, BC)

Neohaematopinus sciuropteri (Alta, BC)

Glaucomys volans (L.)

Southern flying

UNIRAMIA

SIPHONAPTERA

Ceratophyllus gallinae (Man)

Canorhinopsylla stanfordi(Ont)

Ctenophthalmus p. pseudagyrtes (Alta, BC,

Man, NB, NS, Ont, PEI, Que, Sask)

Epitedia faceta (Ont, Que)

Opisodasys pseudarctomys (Alta, BC, Man,

NS, Ont, Que)

Orchopeas $h$. howardi (Alta, Ont)

O. leucopus (Alta, BC, Man, NB, Nfld, NS,

NWT, Ont, Que, Sask)

Oropsylla arctomys (Alta, BC, Man, NB,

NWT, Ont, Que, Sask)

Glaucomys volans volans (L.)

UNIRAMIA

SIPHONAPTERA

Cornorhinopsylla stanfordi(Ont)

Epitedia faceta (Ont, Que)

Orchopeas h. howardii (Alta, Ont)

O. leucopus (Alta, BC, Man, NB, Nfld, NS,

NWT, Ont, Que, Sask)

Glaucomys spp.

Syn.: Sciuropterus spp.

UNIRAMIA

SIPHONAPTERA

Epitedia faceta (Ont, Que)

Megarthroglossus p. procus (BC)

Monopsyllus vison (Alta, BC, Man, Nfld, NS,

NWT, Ont, Que, Sask, YT) 
Opisodasys pseudarctomys (Alta, BC, Man, NS, Ont, Que)

Orchopeas c. caedens (Alta, BC, Man, Que, YT)

O. c. durus (Alta, BC, NB, Nfld, NWT, Ont, Que, Sask)

O. leucopus (Alta, BC, Man, NB, Nfld, NS, NWT, Ont, Que, Sask)

Marmota caligata (Eschscholtz) Hoary marmot CHELICERATA

\section{ACARI}

Dermacentor andersoni (Alta, BC)

UNIRAMIA

SIPHONAPTERA

Monopsyllus ciliatus protinus $(\mathrm{BC})$

Oropsylla arctomys (Alta, BC, Man, NB,

Ont, Que, Sask)

Thrassis s. spenceri (Alta,BC)

Marmota caligata cascadensis Howell

UNIRAMIA

SIPHONAPTERA

Thrassis s. spenceri (Alta, BC)

Marmota caligata okanagana (King)

UNIRAMIA

SIPHONAPTERA

Oropsylla arctomys $(\mathrm{BC})$

Thrassis s. spenceri (Alta, BC)

SIPHUNCULATA

Neohaematopinus marmotae (BC)

Marmota flaviventris (Audubon and Bachman)

Yellow-bellied marmot

UNIRAMIA

SIPHONAPTERA

Thrassis acamantis (Alta, BC)

Marmota flaviventris avara (Bangs)

CHELICERATA

ACARI

Dermacentor andersoni $(\mathrm{BC})$

UNIRAMIA

SIPHONAPTERA

Oropsylla acamantis (BC)

O. idahoensis (Alta, BC, YT)

Thrassis acamantis (Alta, BC)

SIPHUNCULATA

Neohaematopinus marmotae (BC)

Marmota monax(L.) Woodchuck

CHELICERATA

ACARI

Androlaelaps sp. (NS)

Dermacentor andersoni (Alta, BC, Ont)

Haemolaelaps glasgowi (Ont)

Ixodes cookei (Ont)

I. texanus (Ont)

UNIRAMIA

SIPHONAPTERA

Opiscrostis bruneri (Alta, Man, Sask)

Oropsylla arctomys (Alta, BC, Man, NB, NS,

NWT, Ont, Que, Sask)
Thrassis s. spenceri (Alta, BC)

SIPHUNCULATA

Enderleinellus marmotae (Ont)

Marmota monax canadensis (Erxleben)

UNIRAMIA

SIPHONAPTERA

Oropsylla arctomys (Alta, BC, Man, NB, NWT, Ont, Que, Sask)

Marmota monax petrensis Howell

UNIRAMIA

SIPHONAPTERA

Oropsylla arctomys (BC)

Thrassis s. spenceri (Alta, BC)

SIPHUNCULATA

Neohaematopinus marmotae (BC)

Marmota monax rufescens Howell

CHELICERATA

ACARI

Ixodes cookei (Ont, Que)

UNIRAMIA

SIPHONAPTERA

Oropsylla arctomys (Alta, BC, Man, NB, NWT, Ont, Que, Sask)

Marmota vancouverensis Swarth

Vancouver

UNIRAMIA marmot

SIPHONAPTERA

Thrassis acamantis $(\mathrm{BC})$

T. s. spenceri (BC)

Marmota spp.

CHELICERATA

ACARI

Dermacentor andersoni (Alta)

UNIRAMIA

SIPHONAPTERA

Oropsylla arctomys (Alta, BC, Man, NB,

NWT, Ont, Que, Sask)

O. idahoensis (Alta)

Thrassis acamantis (Alta, BC)

T. s. spenceri (Alta, BC)

Sciurus carolinensis Gmelin

Gray squirrel

Syn.: Sciurus carolinensis leucotis of Judd (1954)

UNIRAMIA

SIPHONAPTERA

Conorhinopsylla stanfordi(Ont)

Megabothris acerbus (Man, Ont)

Monopsyllus vison (Alta, BC, Man, Nfld, NS,

NWT, Ont, Que, Sask, YT)

Orchopeas caedens durus (Alta, BC, NB,

Nfld, NWT, Ont, Que, Sask)

O. h. howardi (Alta, Ont)

O. nepos (BC)

SIPHUNCULATA

Neohaematopinus sciurinus (Ont)

Sciurus carolinensis pennsylvanicus Ord UNIRAMIA 
SIPHUNCULATA

Neohaematopinus sciurinus (BC)

Sciurus niger $\mathrm{L}$.

Fox squirrel

UNIRAMIA

SIPHONAPTERA

Orchopeas h. howardi (Alta,Ont)

Sciurus spp.

UNIRAMIA

SIPHONAPTERA

Catallagia charlottensis (BC)

Megarthroglossus divisus exsecatus (BC)

Orchopeas h. howardi (Alta, Ont)

O. sexdentatus agilis (Alta, BC, YT)

Spermophilus columbianus (Ord)

Columbian

CHELICERATA

ACARI

Dermacentor andersoni (Alta, BC)

Ixodes sculptus (Alta)

UNIRAMIA

SIPHONAPTERA

Callistopsyllus t. terinus (BC)

Catallagia charlottensis (BC)

C. decipiens (Alta, BC, Man, Sask, YT)

Hystrichopsylla dippiei spinata (BC)

H. d. truncata $(\mathrm{BC})$

Malaraeus telchinus (BC)

Monopsyllus e. eumolpi (Alta, BC, Man,

NWT, Ont, Sask, YT)

Neopsylla inopina (Alta, BC, Sask)

Opisocrostis bruneri (Alta)

O. t. tuberculatus (Alta, BC, Sask, YT)

Oropsylla arctomys (Alta, BC, Man, NB,

NWT, Ont, Que, Sask)

O. idahoensis (Alta, BC, YT)

$O$. petiolatus (Alta)

O. t. tuberculata (Alta)

Tamiophila grandis (Alta)

Thrassis petiolatus (Alta, BC)

SIPHUNCULATA

Enderleinellus suturalis (Alta)

Neohaematopinus laeviusculus (Alta)

Spermophilus columbianus columbianus (Ord)

Syn.: Citellus columbianus columbianus of Holland (1944)

CHELICERATA

ACARI

Dermacentor andersoni (BC)

UNIRAMIA

SIPHONAPTERA

Foxella ignota recula $(\mathrm{BC})$

Megabothris abantis (Alta, BC)

Monopsyllus eumolpi (Alta, BC, Ont, Sask)

M. vison (Alta, BC, Man, Nfld, NS, NWT,

Ont, Que, Sask, YT)

Neopsylla inopina (Alta, BC, Sask)

Opiscrostis t. tuberculatus (BC)

Oropsylla idahoensis (Alta, BC)

O. petiolatus $(\mathrm{BC})$
O. tuberculatus (Alta, BC)

Rhadinopsylla fraterna $(\mathrm{BC})$

R. s. sectilis (BC)

Thrassis acamantis $(\mathrm{BC})$

T. petiolatus $(\mathrm{BC})$

SIPHUNCULATA

Neohaematopinus laeviusculus (BC)

Spermophilus franklinii (Sabine) Franklin's ground

Syn.: Citellus franklinii (Sabine)

squirrel

\section{CHELICERATA}

ACARI

Dermacentor variabilis (Man)

Ixodes sculptus (Alta)

UNIRAMIA

SIPHONAPTERA

Hystrichopsylla d. dippiei (Alta, Man, Ont)

Megabothris atrox (Alta, Man, Ont)

M. quirini (Alta, BC, NWT, Ont)

Neopsylla inopina (Alta, BC, Sask)

Opisocrostis bruneri (Alta, Man, Sask)

O. labis (Alta, Sask)

O. t. tuberculatus (Alta, Sask)

Orchopeas c. caedens (Alta, BC, NWT, Sask,

YT)

Oropsylla arctomys (Alta, BC, Man, NB,

NWT, Ont, Que, Sask)

$O$. bacchi (unspecified)

$O$. rupestris (Alta, Man, Sask)

Thrassis b. bacchi (Alta, Man, Sask)

SIPHUNCULATA

Enderleinellus suturalis (Alta)

Spermophilus lateralis (Say)

Golden-mantled

UNIRAMIA

SIPHONAPTERA

Amalaraeus dissimilis (Alta, BC, NWT, YT)

Oropsylla idahoensis (Alta, YT)

Rhadinopsylla difficilis (BC)

Thrassis petiolatus (Alta, BC)

Spermophilus lateralis tescorum (Hollister)

UNIRAMIA

SIPHONAPTERA

Oropsylla idahoensis (Alta)

Spermophilus parryii (Richardson) Arctic ground squirrel

Syn.: Spermophilus undulatus of Spencer (1966)

S. u. parryii of Spencer (1966)

UNIRAMIA

SIPHONAPTERA

Megabothris groenlandicus (Nfld, NWT, YT)

M. quirini (Alta, BC, Man, NB, Nfld, NS,

NWT, Ont, Que, Sask, YT)

Ceratophyllus lunatus tundrensis (Man, Nfld, NWT, Que, YT)

Opisocrostis t. tuberculatus (Alta, BC, Sask, YT)

Orchopeas c. caedens (Alta, BC, Man, Que,

YT) 
Oropsylla alaskensis (NWT, YT)

O. idahoensis (Alta, BC, YT)

O. rupestris (Alta, Man, Sask, YT)

Rhadinopsylla linta (BC, NWT, YT)

SIPHUNCULATA

Neohaematopinus laeviusculus (NWT)

Spermophilus parryii parryii (Richardson)

UNIRAMIA

SIPHONAPTERA

Ceratophyllus lunatus tundrensis (NWT,

Que)

Oropsylla alaskensis (NWT)

Spermophilus parryii plesius Osgood

Syn.: Spermophilus undulatus plesius

UNIRAMIA

SIPHONAPTERA

Monopsyllus vison (Alta, BC, Man, Nfld, NS,

NWT, Ont, Que, Sask, YT)

Oropsylla idahoensis (Alta, BC, YT)

Spermophilus richardsonii (Sabine) Richardson's

Syn.: Citellus richardsonii (Sabine)

ground squirrel

CHELICERATA

ACARI

Dermacentor andersoni (Alta)

Haemaphysalis leporis-palustris (Alta)

Hirstionyssus occidentalis (Alta)

Ixodes kingi (Alta)

I. sculptus (Alta)

Liponyssus montanus (Man)

L. occidentalis (Man)

Pneumonyssus spp. (Alta)

UNIRAMIA

DIPTERA

Cuterebra grisea (Sask)

SIPHONAPTERA

Catallagia decipiens (Alta, BC, Man, Sask,

YT)

Epitedia inopina (Alta)

E. w. wenmanni (Alta, BC, Man, NB, Nfld,

NS, NWT, Ont, Que, Sask, YT)

Hystrichopsylla d. dippiei (Alta, Man, Ont,

Sask)

Nearctopsylla brooksi (Alta, BC)

Neopsylla inopina (Alta, BC, Sask)

Opisocrostis bruneri (Alta, Man, Sask)

O. labis (Alta, Sask)

O. t. tuberculatus (Alta, Sask, YT)

Oropsylla arctomys (Alta)

O. idahoensis (Alta)

O. rupestris (Alta, Man, Sask, YT)

Rhadinopsylla fraterna (Alta, BC, Sask)

Tamiophila grandis (Alta)

Thrassis b. bacchi (Alta, Man, Sask)

T. petiolatus (Alta, BC)

SIPHUNCULATA

Enderleinellus suturalis (Alta)

Neohaematopinus laeviusculus (Alta)
Spermophilus richardsonii richardsonii (Sabine)

Syn.: Citellus richardsonii richardsonii (Sabine)

UNIRAMIA

SIPHONAPTERA

Ctenophthalmus p. pseudagyrtes (Alta, Man, Ont, Sask)

Epitedia w. wenmanni (Alta, BC, Man, NB,

NWT, Ont, Sask)

Hystrichopsylla d. dippiei (Alta, Sask)

Megabothris asio megacolpus (Sask)

M. lucifer (Alta, BC, Sask)

Neopsylla inopina (Alta, Sask)

Opisocrostis bruneri (Alta, Man, Sask)

O. labis (Alta, Sask)

O. t. tuberculatus (Alta, BC, Sask, YT)

Oropsylla idahoensis (Alta, BC)

$O$. rupestris (Alta, Man, Sask, YT)

Peromyscopsylla selenis (Alta, BC, Man,

NWT, Sask)

Rhadinopsylla fraterna (Alta, BC, Sask)

Thrassis b. bacchi (Alta, Man, Sask, unspecified)

Spermophilus saturatus (Rhoads) Cascade goldenmantled ground squirrel

UNIRAMIA

SIPHONAPTERA

Oropsylla idahoensis (Alta, BC, YT)

Spermophilus tridecemlineatus (Mitchell) Thirteenlined ground squirrel

Syn.: Citellus tridecemlineatus (Mitchell)

CHELICERATA

ACARI

Dermacentor variabilis (Man)

Liponyssus montanus (Man)

L. occidentalis (Man)

UNIRAMIA

SIPHONAPTERA

Ceratophyllus bruneri (Man)

Opisocrostis bruneri (Alta, Man, Sask)

O. t. tuberculatus (Alta, BC, Sask)

Oropsylla idahoensis (Alta, BC)

Thrassis b. bacchi (Alta, Man, Sask)

Spermophilus tridecemlineatus tridecemlineatus

Syn.: Citellus t. tridecemlineatus (Mitchell)

UNIRAMIA

SIPHONAPTERA

Opisocrostis bruneri (Alta, Man, Sask)

O. t. tuberculatus (Alta, Sask)

Thrassis b. bacchi (Alta, Sask)

Spermophilus spp.

Syn.: Citellus spp.

CHELICERATA

ACARI

Dermacentor andersoni (BC, Man)

UNIRAMIA

SIPHONAPTERA

Monopsyllus vison (Alta, BC, Man, Nfld, NS,

NWT, Ont, Que, Sask, YT) 
Oropsylla alaskensis (NWT, YT)

O. idahoensis (Alta, BC, YT)

O. rupestris (Alta, Sask)

Rhadinopsylla linta (BC, NWT, YT)

Thrassis petiolatus (Alta, BC)

SIPHUNCULATA

Linognathoides montanus (Canada)

Tamiasciurus douglasii (Bachman) Douglas'squirrel CHELICERATA

ACARI

Ixodes spinapalpus $(\mathrm{BC})$

UNIRAMIA

SIPHONAPTERA

Hystrichopsylla o. occidentalis (BC)

Megarthroglossus d. divisus (BC)

M. p. procus $(\mathrm{BC})$

Monopsyllus ciliatus protinus (BC)

$M$. wagneri ophidius (BC)

Opisodasys vesperalis $(\mathrm{BC})$

Orchopeas caedens durus (Alta, BC, NB,

Nfld, NWT, Ont, Que, Sask)

O. nepos (BC)

Tamiasciurus douglasii mollipilosus (Audubon and Bachman)

Syn.: Tamiasciurus douglasii cascadensis (Allen)

CHELICERATA

ACARI

Ixodes angustus $(\mathrm{BC})$

UNIRAMIA

SIPHONAPTERA

Dasypsyllus gallinulae perpinnatus $(\mathrm{BC})$

Megarthroglossus p. procus (BC)

Monopsyllus ciliatus protinus (BC)

Nosopsyllus fasciatus (BC, Ont, Que, Sask)

Orchopeas caedens durus (Alta, BC, NB,

Nfld, NWT, Ont, Que, Sask)

O. nepos (BC)

Rhadinopsylla sectilis goodi $(\mathrm{BC})$

SIPHUNCULATA

Hoplopleura sciuricola (BC)

Tamiasciurus hudsonicus (Erxleben)

Syn.: Sciurus hudsonicus Erxleben

Red squirrel

CHELICERATA

ACARI

Dermacarus hypudaei septentrionalis (Que)

D. newyorkensis (Que)

D. sciurinus (Que)

Dermacentor variabilis (NS)

Gliricoptes spp. (Que)

Glycephagidae gen. spp. (Ont)

Haemaphysalis leporis-palustris (NS)

Haemolaelaps glasgowi (Ont)

Ixodes angustus (Alta)

I. marxi (NS, Ont, Que)

I. muris (NS)

Labidophorids (Ont)

Neotrombicula microti(NS)

Trombicula spp. (Que)

Trombiculidae gen. spp. (Ont)

Zumptiella bakeri (Que)
UNIRAMIA

SIPHONAPTERA

Catallagia charlottensis (BC)

C. decipiens (Alta, BC, Man, Sask, YT)

Ceratophyllus lunatus tundrensis (Man, Nfld,

NWT, Que, YT)

C. niger (BC)

Ctenophthalmus p. pseudagyrtes (Alta, BC,

Man, NB, NS, Ont, PEI, Que, Sask)

Epitedia w. wenmanni (Alta, BC, Man, NB,

Nfld, NS, NWT, Ont, Que, Sask, YT)

Hystrichopsylla dippiei ssp. (NB, Ont)

H. d. truncata $(\mathrm{BC})$

Megabothris abantis (Alta, BC, YT)

M. asio megacolpus (Alta, BC, Man, NWT,

Ont, Que, Sask, YT)

M. quirini (Alta, BC, Man, NB, Nfld, NS,

NWT, Ont, Que, Sask, YT)

Megarthroglossus d. divisus (Alta)

M. d. exsecatus (BC)

Monopsyllus ciliatus protinus (BC)

M. eumolpi (Alta, BC, Ont, Sask)

M. e. eumolpi (Alta, BC, Man, NWT, Ont,

Sask, YT)

M. vison (Alta, BC, Man, NB, Nfld, NS,

NWT, Ont, Que, Sask, YT)

M. w. wagneri (Alta, BC)

Opisocrostis bruneri (Man)

O. t. tuberculatus (Alta, BC, Sask, YT)

Opisodasys pseudarctomys (Alta, BC, Man,

NS, Ont, Que)

Orchopeas caedens (NWT, Ont)

O. c. caedens (Alta, BC, Man, NWT, Que,

Sask, YT)

O. c. durus (BC, Nfld, NS, NWT)

O. h. howardi (Alta, Ont)

O. leucopus (Que)

O. sexdentatus agilis (Alta, BC, YT)

Oropsylla arctomys (Alta, BC, Man, NB,

NWT, Ont, Que, Sask)

Peromyscopsylla catatina (Man, Nfld, NS,

Ont)

P. selenis (Alta, BC, Man, NWT, Sask, YT)

Rhadinopsylla arborea (Alta, BC)

R. fraterna (Alta, BC, Sask)

$R$. linta (BC, NWT, YT)

R. s. sectilis $(\mathrm{BC})$

Stenoponia americana (NB)

Tarsopsylla octodecimdentata coloradensis

(Alta, BC, Man, NWT, Ont, Sask YT)

Trichopsylla vison (Alta)

SIPHUNCULATA

Enderleinellus nitschi(Ont, Que)

Hoplopleura hesperomydis (Que)

H. sciuricola (Alta, BC)

Neohaematopinus sciurinus (Alta)

N. semifasciatus (Ont)

Tamiasciurus hudsonicus baileyi (Allen)

Syn.: Sciurus richardsoni baileyi of Brown (1944)

UNIRAMIA

SIPHONAPTERA 
Malaraeus euphorbi (Alta, BC, NWT, Sask)

Megarthroglossus d. divisus (Alta)

Monopsyllus vison (Alta, BC, Man, Nfld, NS,

NWT, Ont, Que, Sask, YT)

Rhadinopsylla arborea (Alta, BC)

Tamiasciurus hudsonicus columbiensis Howell

UNIRAMIA

SIPHONAPTERA

Megabothris quirini (Alta, BC, NWT, Ont)

Megarthroglossus divisus exsecatus (BC)

Monopsyllus vison (Alta, BC, Man, Nfld, NS,

NWT, Ont, Que, Sask, YT)

Orchopeas caedens durus (BC, Nfld, NWT)

SIPHUNCULATA

Neohaematopinus sciurinus (BC)

Solenopotes ferrisi (BC)

Tamiasciurus hudsonicus gymnicus (Bangs)

CHELICERATA

ACARI

Haemaphysalis leporis-palustris (NS)

UNIRAMIA

SIPHONAPTERA

Ctenophthalmus p. pseudagyrtes (NB)

Epitedia w. wenmanni (NB)

Megabothris quirini (NB)

Monopsyllus vison (NB)

Nearctopsylla g. genalis (NB)

Orchopeas caedens durus (NB)

O. h. howardi (Que)

Tamiasciurus hudsonicus hudsonicus (Erxleben)

UNIRAMIA

SIPHONAPTERA

Megabothris quirini (Alta, BC, Man, NWT,

Ont)

Monopsyllus vison (Alta, BC, Man, Nfld, NS,

NWT, Ont, Que, Sask, YT)

Opisocrostis bruneri (Alta, Man, Sask)

Orchopeas caedens durus (Alta, BC, NB,

Nfld, NWT, Ont, Que, Sask)

O. leucopus(Man)

Peromyscopsylla catatina(Ont)

Tamiasciurus hudsonicus lanuginosus (Bachman)

UNIRAMIA

SIPHONAPTERA

Monopsyllus ciliatus protinus $(\mathrm{BC})$

SIPHUNCULATA

Neohaematopinus sciurinus $(\mathrm{BC})$

Tamiasciurus hudsonicus laurentianus Anderson

UNIRAMIA

SIPHONAPTERA

Megabothris quirini (Que)

Tamiasciurus hudsonicus loquax (Bangs)

CHELICERATA

ACARI

Ixodes marxi (Ont)

UNIRAMIA

SIPHONAPTERA

Orchopeas h. howardii (Ont)
Tamiasciurus hudsonicus minnesota (Allen)

UNIRAMIA

SIPHONAPTERA

Monopsyllus vison (Alta, BC, Man, Nfld, NS,

NWT, Ont, Que, Sask, YT)

Tamiasciurus hudsonicus picatus (Swarth)

UNIRAMIA

SIPHONAPTERA

Ceratophyllus petrochelidoni $(\mathrm{BC})$

Monopsyllus ciliatus protinus (BC)

M. vison (Alta, BC, Man, Nfld, NS, NWT,

Ont, Que, Sask, YT)

Tamiasciurus hudsonicus preblei Howell

UNIRAMIA

SIPHONAPTERA

Ceratophyllus garei (Alta, BC, Man, NWT,

Que, YT)

Monopsyllus vison (Alta, BC, Man, Nfld, NS,

NWT, Ont, Que, Sask, YT)

Orchopeas c. caedens (Alta, BC, NWT, Sask,

YT)

O. c. durus (NWT)

O. sexdentatus agilis (Alta,YT)

Tamiasciurus hudsonicus richardsoni (Bachman)

Syn.: Sciurus hudsonicus richardsoni Bachman

UNIRAMIA

SIPHONAPTERA

Megarthroglossus d. divisus (Alta)

Monopsyllus vison (Alta, BC, Man, Nfld, NS,

NWT, Ont, Que, Sask, YT)

Orchopeas sexdentatus agilis (Alta)

Tamiasciurus hudsonicus streatori (Allen)

CHELICERATA

ACARI

Ixodes hearlei $(\mathrm{BC})$

UNIRAMIA

SIPHONAPTERA

Atyphloceras m. multidentatus (BC)

Epitedia w. wenmanni (Alta, BC, Man, NB,

NWT, Ont, Sask)

Megabothris abantis (BC)

Megarthroglossus divisus exsecatus (BC)

Monopsyllus eumolpi (Alta, BC, Ont, Sask)

M. vison (Alta, BC, Man, Nfld, NS, NWT,

Ont, Que, Sask, YT)

Opisodasys pseudarctomys (Alta, Ont)

Orchopeas caedens durus (Alta, BC, NB,

Nfld, NWT, Ont, Que, Sask)

Rhadinopsylla s. sectilis (BC)

Thrassis acamantis (Alta)

SIPHUNCULATA

Hoplopleura sciuricola (BC)

Neohaematopinus sciurinus (BC)

Tamiascurus hudsonicus ungavensis Anderson

UNIRAMIA

SIPHONAPTERA

Monopsyllus vison (Alta, BC, Man, Nfld, NS,

NWT, Ont, Que, Sask, YT) 
Orchopeas caedens durus (Nfld, Que)

Tamiasciurus spp.

CHELICERATA

ACARI

Ixodes pacificus $(\mathrm{BC})$

UNIRAMIA

SIPHONAPTERA

Atyphloceras m. multidentatus (BC)

Cororhinopsylla stanfordi (Ont)

Ctenophthalmus p. pseudagyrtes (Alta, BC,

Man, NB, NS, Ont, PEI, Que, Sask)

Epitedia w. wenmanni (Alta, BC, Man, NB,

Nfld, NS, NWT, Ont, Que, Sask, YT)

Megabothris quirini (Alta, BC, Man, NB,

Nfld, NS, NWT, Ont, Que, Sask, YT)

Megarthroglossus divisus exsecatus (BC)

Monopsyllus cileatus protinus (BC)

M. e. eumolpi (Alta, BC, Man, NWT, Ont,

Sask, YT)

M. vison (Alta, BC, Man, Nfld, NS, NWT,

Ont, Que, Sask, YT)

Orchopeas c. caedens (Alta, BC, Man, Que,

YT)

O. c. durus (Alta, BC, NB, Nfld, NWT, Ont,

Que, Sask)

O. nepos (BC)

Tarsopsylla octodecimdentata coloradensis

(Alta, BC, Man, NWT, Ont, Sask, YT)

Tamias striatus (L.)

CHELICERATA

Eastern chipmunk

ACARI

Dermacarus hylandi (NB)

Dermacentor variabilis (Man, NS)

Euschoengastia setosa (Que)

Haemaphysalis leporis-palustris (PEI)

Haemolaelaps glasgowi (Ont)

Ixodes angustus (Ont)

I. muris (NS)

Miyatrombicula esoensis (NB)

Myocoptes spp. (Que)

Neotrombicula harperi (NB)

Sarcoptidae gen. spp. (Ont)

Trombiculidae gen. spp. (Ont)

\section{UNIRAMIA}

DIPTERA

Cuterebra emasculator (Ont)

Cuterebra sp. (Ont)

SIPHONAPTERA

Ctenophthalmus p. pseudagyrtes (Alta, BC,

Man, NB, NS, Ont, PEI, Que, Sask)

Hoplopleura erratica (Ont, Que)

Megabothris acerbus (Man, Ont)

M. asio megacolpus (Man)

M. quirini (Alta, BC, Man, NB, Nfld, NS,

NWT, Ont, Que, Sask, YT)

Monopsyllus e. eumolpi (Alta, BC, Man,

NWT, Ont, Sask, YT)

$M$. vison (Ont)

Orchopeas caedens durus (Alta, BC, NB,

Nfld, NWT, Ont, Que, Sask)
O. leucopus (Alta, BC, Man, NB, Nfld, NS, NWT, Ont, Que, Sask, YT)

Tamiophila grandis (Ont, Que)

SIPHUNCULATA

Hoplopleura erratica (Ont, Que)

Neohaematopinus sciuropteri (Ont)

Tamias striatus griseus Mearns

UNIRAMIA

SIPHONAPTERA

Ctenophthalmus p. pseudagyrtes (Alta, BC,

Man, NB, NS, Ont, PEI, Que, Sask)

Megabothris acerbus (Ont)

M. asio megacolpus (Alta, BC, Man, NWT,

Ont, Que, Sask, YT)

M. quirini (Alta, BC, Man, NB, Nfld, NS,

NWT, Ont, Que, Sask, YT)

Tamiophila grandis (Ont)

Tamias striatus lysteri (Richardson)

UNIRAMIA

DIPTERA

Cuterebra emasculator (unspecified)

SIPHONAPTERA

Epitedia w. wenmanni (Ont)

Megabothris acerbus (Ont)

Orchopeas h. howardi(Ont)

Tamiophila grandis (Ont)

Trichopsylla acerbus (Ont)

Tamias spp.

UNIRAMIA

SIPHONAPTERA

Megabothris acerbus (Man, Ont)

Orchopeas leucopus (Alta, BC, Man, NB,

Nfld, NS, NWT, Ont, Que, Sask)

\section{SCIURIDAE OF UNDETERMINED SPECIES}

Chipmunk

CHELICERATA

ACARI

Dermacentor andersoni (Alta, BC, Ont)

Eulaelaps stabularis (Ont)

Haemaphysalis leporis-palustris (BC)

Ixodes angustus $(\mathrm{BC})$

I. $\operatorname{marxi}(\mathrm{BC})$

Laelaps pedalis (Ont)

UNIRAMIA

SIPHONAPTERA

Megabothris abantis (Alta, BC, YT)

M. acerbus (Man, Ont)

Monopsyllus e. eumolpi (Alta, BC, Man,

NWT, Ont, Sask, YT)

Opisodasys k. keeni (Alta, BC)

Orchopeas caedens durus (Alta, BC, NB,

Nfld, NWT, Ont, Que, Sask)

Tamiophila grandis (Ont)

Thrassis acamantis (Alta, BC)

Gopher

UNIRAMIA

DIPTERA 
Cuterebra sp. (Sask)

SIPHONAPTERA

Catallagia decipiens (Alta, BC, Man, Sask,

YT)

Foxella ignota recula $(\mathrm{BC})$

Groundhog

CHELICERATA

ACARI

Dermacentor andersoni (BC, Ont)

Haemaphysalis leporis-palustris (BC)

Ixodes cookei (BC, Ont)

Laelaps propheticus (Ont)

UNIRAMIA

SIPHONAPTERA

Siphonaptera gen. spp. (BC)

Thrassis acamantis (Alta, BC)

Ground squirrel

CHELICERATA

ACARI

Dermacentor andersoni (Alta, BC)

Ixodes sculptus (Sask)

UNIRAMIA

SIPHONAPTERA

Hystrichopsylla dippiei (Alta)

Neopsylla inopina (Alta)

Opisocrostis labis (Alta)

Rhadinopsylla fraterna (Alta)

Siphonaptera gen. spp. (BC)

Marmot

UNIRAMIA

SIPHONAPTERA

Siphonaptera gen. spp. (BC)

Red squirrel

UNIRAMIA

SIPHONAPTERA

Monopsyllus ciliatus protinus (BC)

M. e. eumolpi (Alta, BC, Man, NWT, Ont,

Sask, YT)

M. vison (Alta, BC, Man, Nfld, NS, NWT,

Ont, Que, Sask, YT)

Orchopeas c. caedens (Alta, BC, Man, Que,

YT)

O. c. durus $(\mathrm{BC})$

Squirrels

CHELICERATA

ACARI

Dermacentor andersoni (BC)

Gemasus spp. (Ont)

Haemaphysalis cinnabarina $(\mathrm{BC})$

H. leporis-palustris (Sask)

Ixodes angustus (BC)

I. californicus $(\mathrm{BC})$

I. cookei (BC)

I. marxi (BC, Ont)

I. spinipalpus $(\mathrm{BC})$

I. texanus $(\mathrm{BC})$

UNIRAMIA

SIPHONAPTERA

Ceratophyllus gallinae (Man)
Megarthroglossus p. procus (BC)

Monopsyllus ciliatus protinus (BC)

M. vison (Alta, BC, Man, NB, Nfld, NS,

NWT, Ont, Que, Sask, YT)

Orchopeas caedens durus (Alta, BC, NB,

Nfld, NWT, Ont, Que, Sask)

Tarsopsylla octodecimdentata coloradensis

(Alta, BC, Man, NWT, Ont, Sask, YT)

Streator's red squirrel

CHELICERATA

ACARI

Liponyssus spp. (Ont)

Neoschongastia blarinae (BC)

Tree squirrel

CHELICERATA

ACARI

Dermacentor andersoni (Alta)

UNIRAMIA

SIPHONAPTERA

Orchopeas c. caedens (Alta, BC, Man, Que, YT)

Family ZAPODIDAE

Napaeozapus insignis (Miller) Woodland jumping

CHELICERATA

ACARI

Androlaelaps fahrenholzi (NS, PEI)

Androlaelaps sp. (NS)

Dermacarus hypudaei (NS)

D. newyorkensis (NB, NS, PEI)

Dermacentor variabilis (NS)

Echinonyssus isabellinus (PEI)

Euryparasitus spp. (NB)

Gliricoptes zapus (NB)

Gliricoptes sp. (Que)

Glycyphagus hypudaei (NB)

Haemogamasus ambulans (NB)

Haemogamasus spp. (NS)

Ixodes angustus (NB)

I. muris (NS)

Laelaps kochi (NS, PEI)

Listrophorus mexicanus (NS, Que)

Miyatrombicula esoensis (PEI)

Myobia sp. (Que)

Neotrombicula harperi (NB, NS, PEI, Que)

N. microti (NS, Que)

Pygmephorus horridus (NB)

P. mahunkai (PEI)

Radfordia ewingi (NB, PEI)

Radfordia sp. (NB)

UNIRAMIA

SIPHONAPTERA

Ctenophthalmus p. pseudagyrtes(NS)

Megabothris a. asio (NS)

M. quirini (Alta, BC, Man, NB, Nfld, NS,

NWT, Ont, Que, Sask, YT)

Orchopeas leucopus (Que)

Peromyscopsylla catatina (Man, NB, NS,

Ont) 
Stenoponia americana (NS)

SIPHUNCULATA

Enderleinellus nitschi (Que)

Napaeozapus insignis frutectanus Jackson

UNIRAMIA

SIPHONAPTERA

Megabothris quirini (Alta, BC, NWT, Ont)

Peromyscopsylla catatina (Ont)

Napeozapus insignis insignis (Miller)

CHELICERATA

ACARI

Ixodes muris (NS)

UNIRAMIA

SIPHONAPTERA

Megabothris quirini (Alta, BC, Man, NB,

Nfld, NS, NWT, Ont, Que, Sask, YT)

Peromyscopsylla catatina (Man, Nfld, NS,

Ont)

Stenoponia americana (Man, NS, Ont, Que)

Napaeozapus spp.

UNIRAMIA

SIPHONAPTERA

Ctenophthalmus p. pseudagyrtes (Alta, BC,

Man, NB, NS, Ont, PEI, Que, Sask)

Megabothris quirini (Alta, BC, Man, NB,

Nfld, NS, NWT, Ont, Que, Sask,YT)

Zapus hudsonius (Zimmerman) Meadow jumping

CHELICERATA

ACARI

Androlaelaps fahrenholzi (NB, NS, PEI)

Androlaelaps sp. (NS)

Dermacarus newyorkensis (NB, NS, PEI)

Dermacentor variabilis (Man, NS)

Echinonyssus isabellinus (PEI)

Haemogamasus ambulans (PEI)

Hyperlaelaps sp. (NS)

Ixodes angustus (NB)

I. muris $(\mathrm{NB}, \mathrm{NS}, \mathrm{PEI})$

Laelaspis sp. (NS)

Listrophorus mexicanus (PEI)

Myobiiidae gen. spp. (Ont)

Neotrombicula harperi (NB, NS, PEI, Que)

N. lipovskyi (NB)

N. microti (NS)

Ololaelaps sp. (NS)

Orycteroxenus soricis (NS)

Pygmephorus mahunkai (PEI)

Radfordia ewingi (NB)

Trombiculidae gen. spp. (Ont)

Xenoryctes latiporus (NB)

UNIRAMIA

SIPHONAPTERA

Catallagia decipiens (Alta, BC, Man, Sask,

YT)

Corrodopsylla c. curvata (Alta, BC, Man,

NB, Nfld, NS, NWT, Ont, Que, Sask, YT)

Megabothris abantis (Alta, BC, YT)

M. asio (NS)
M. a. asio (Nfld, Ont, Que, Sask, YT)

M. a. megacolpus (Alta, BC, Man, NWT,

Ont, Que, Sask, YT)

M. quirini (Alta, BC, Man, NB, Nfld, NS,

NWT, Ont, Que, Sask, YT))

Peromyscopsylla selenis (Alta, BC, Man,

NWT, Sask, YT)

Zapus hudsonius acadicus (Dawson)

CHELICERATA

ACARI

Dermacentor variabilis (NS)

Ixodes muris (NS)

Zapus hudsonius canadensis (Davies)

Syn.: Zapus hudsonius ontarioensis Anderson

CHELICERATA

ACARI

Androlaelaps fahrenholzi (Ont)

Haemogamasus hirsutus (Ont)

Zapus princeps Allen Western jumping

UNIRAMIA

SIPHONAPTERA

Hystrichopsylla d. dippiei (Alta, Sask)

Megabothris quirini (Alta, BC, Man, NB,

Nfld, NS, NWT, Ont, Que, Sask, YT)

Peromyscopsylla selenis (Alta, BC, Man,

NWT, Sask, YT)

Zapus princeps idahoensis Davis

UNIRAMIA

SIPHONAPTERA

Catallagia decipiens (Alta, BC, Sask)

Megabothris abantis (Alta, BC)

Zapus princeps minor Preble

UNIRAMIA

SIPHONAPTERA

Epitedia w. wenmanni (Alta, BC, Man, NB,

NWT, Ont, Sask)

Hystrichopsylla d. dippiei (Alta, Sask)

Megabothris quirini (Alta, BC, NWT, Ont)

Monopsyllus eumolpi (Alta, BC, Ont, Sask)

Zapus trinotatus trinotatus Rhoads Pacific jumping

UNIRAMIA

SIPHONAPTERA

Hystrichopsylla o. occidentalis (BC)

Megabothris abantis (Alta, BC)

Zapus spp.

UNIRAMIA

SIPHONAPTERA

Catallagia decipiens (Alta, BC, Man, Sask,

YT)

Corrodopsylla c. curvata (Alta)

Ctenophthalmus p. pseudagyrtes (Que)

Epitedia scapani $(\mathrm{BC})$

Malaraeus telchinus (Alta, BC)

Megabothris abantis (Alta, BC, YT)

M. a. asio (NB, Nfld, NS, Ont, PEI, Que) 
M. a. megacolpus (Man)

M. quirini (Alta, BC, Man, NB, Nfld, NS, NWT, Ont, Que, Sask, YT)

Monopsyllus wagneri systaltus (Alta, Man, Ont, Sask)

M. w. wagneri (Alta, BC)

Opisodasys k. keeni (Alta, BC)

Peromyscopsylla selenis (Alta)

\section{RODENTIA OF UNDETERMINED SPECIES}

\author{
Rodents \\ CHELICERATA \\ ACARI \\ Dermacentor venustus $(\mathrm{BC})$ \\ Ixodidae gen. sp. (Alta, BC) \\ UNIRAMIA \\ SIPHONAPTERA
}

Rectofrontia fraterna $(\mathrm{BC})$

Siphonaptera gen. spp. (BC)

MAMMALIA OF UNDETERMINED SPECIES

Mammalia gen. spp.

CHELICERATA

ACARI

Dermacentor albipictus (BC)

D. andersoni (Alta)

D. variabilis (NS)

Haemaphysalis expositicius (Man)

UNIRAMIA

DIPTERA

Cuterebra americana var. polita $(\mathrm{BC})$

C. fasciata (BC, NB)

C. similis $(\mathrm{BC})$

C. tenebrosa $(\mathrm{BC})$

SIPHONAPTERA

Nosopsyllus fasciatus (Ont) 


\section{References}

ADDISON, E.M., F.J. JOHNSON, AND A. FYVIE. 1979. Dermacentor albipictus on moose (Alces alces) in Ontario. Journal of Wildlife Diseases 15: 281-284.

ADDISON, E.M., M.J. PYBUS, AND H.J. RIETVELD. 1978. Helminth and arthropod parasites of black bear, Ursus americanus, in central Ontario. Canadian Journal of Zoology 56: 2122-2126.

ANON. 1930. Experimental fur farm. Report of the Ontario Department of Game and Fisheries for 1929 23: 9-12.

ARNETT, R.H. JR. 1968. The beetles of the United States. The American Entomological Institute. Ann Arbor, Michigan. $1112 \mathrm{pp}$.

ARNETT, R.H., JR., N.M. DOWNIE, AND H.E. JAQUES. 1980. How to know the beetles. 2nd ed. Wm. C. Brown Co. Dubuque, Iowa. 416 pp.

ARTHUR, D.R., AND K.R. SNOW. 1968. Ixodes pacificus Cooley and Kohls, 1943: Its life-history and occurrence. Parasitology 58: 893-906.

BAKER, C.F. 1896. A new Pulex from Queen Charlotte Islands. The Canadian Entomologist 28: 234.

1898. Notes on Siphonaptera, with descriptions of four new species. Journal of the New York Entomological Society 6: 53-56.

1904. A revision of American Siphonaptera or fleas, together with a complete list and bibliography of the group. Proceedings of the United States National Museum 27: 365-469.

BAKER, J.A. 1946. A rickettsial infection in Canadian voles. Journal of Experimental Medicine 84: 37-50.

BANFIELD, A.W.F. 1954. Preliminary investigation of the barren ground caribou. Part II. Life history, ecology, and utilization. Wildlife Management Bulletin 1 (10B): $32-43$.

1956. An investigation of ticks as disease vectors in Banff National Park, Alberta. Canadian Journal of Zoology 34: 417-423.

BANKS, N. 1909. New Canadian mites. [Arachnoidea, Acarina]. Proceedings of the Entomological Society of Washington 11: 133-143.

BENNETT, G.F. 1955. Studies on Cuterebra emasculator Fitch 1856 (Diptera: Cuterebridae) and a discussion of the status of the genus Cephenomyia Ltr. 1818. Canadian Journal of Zoology 33: 75-98.

1962. On the biology of Cephenomyia phobifera (Diptera: Oestridae), the pharyngeal bot of the whitetailed deer, Odocoileus virginianus. Canadian Journal of Zoology 40: 1195-1210.

1972. Further studies on the chipmunk warble, Cuterebra emasculator (Diptera: Cuterebridae). Canadian Journal of Zoology 50: 861-864.

1973. Some effects of Cuterebra emasculator Fitch (Diptera: Cuterebridae) on the blood and activity of its host, the eastern chipmunk. Journal of Wildlife Diseases 9: 85-93.
BENNETT, G.F., AND C.W. SABROSKY. 1962. The Nearctic species of the genus Cephenomyia (Diptera: Oestridae). Canadian Journal of Zoology 40: 431-448.

BEQUAERT, J.C. 1935. The American species of Lipoptena (Diptera, Hippoboscidae). Bulletin of the Brooklyn Entomological Society 30: 170

1937. Notes on Hippoboscidae. 5. The American species of Lipoptena. Bulletin of the Brooklyn Entomological Society 32: 91-101.

1942. A monograph of the Melophaginae, or ked-flies, of sheep, goat, deer and antelopes (Diptera,

Hippoboscidae). Entomologica Americana 22: 1-220.

1945. The ticks, or Ixodoidea, of the northeastern United States and eastern Canada. Entomologica Americana 25: 121-224.

1957. The Hippoboscidae or louse-flies (Diptera) of mammals and birds. Part II. Taxonomy, evolution and revision of American genera and species. Entomologia Americana 36: 417-611.

BLOOD, D.A. 1963. Parasites from California bighorn sheep in southern British Columbia. Canadian Journal of Zoology 41: 913-918.

BOONSTRA, R. 1977. Effect of the parasite Wohlfahrtia vigil on Microtus townsendii populations. Canadian Journal of Zoology 55: 1057-1060.

BROWN, J.H. 1944. The fleas (Siphonaptera) of Alberta, with a list of the known vectors of sylvatic plague. Annals of the Entomological Society of America 37: 207-213.

BROWN, J.H., AND G.M. KOHLS. 1950. The ticks of Alberta with special reference to distribution. Canadian Journal of Research (series D) 28: 197-205.

BROWN, N.R. 1955. Additional host and distribution records of some eastern Canadian Siphonaptera. Canadian Entomologist 87: 362-365.

1968. Notes on the Siphonaptera of New Brunswick. Canadian Entomologist 100: 486-498.

BRUCE, E.A. 1920. Tick paralysis in British Columbia. Dominion of Canada, Department of Agriculture, Bulletin Number 133: 1-7.

BUCKELL, E.R. 1934. Notes on ticks and insect parasites of game animals in British Columbia. Proceedings of the Entomological Society of British Columbia 31: 10-16.

BUCKNER, C.H. 1964. Fleas (Siphonaptera) of Manitoba mammals. Canadian Entomologist 96: 850-856.

BUCKNER, C.H., AND G.G. BLASKO. 1969. Additional range and host records of the fleas (Siphonaptera) of Manitoba. Manitoba Entomologist 3: 65-69.

BURACHYNSKY, V.I., AND T.D. GALLOWAY. 1985. Seasonal dynamics and distribution of American dog tick, Dermacentor variabilis (Say), larvae and nymphs at Bird Hill Park, Manitoba. Canadian Journal of Zoology 63: 2748-2755.

CAMERON, A.E. 1926. The occurrence of Cuterebra 
(Diptera: Oestridae) in western Canada. Parasitology 18: 430-435.

CAMERON, A.E., AND J.S. FULTON. 1927. A local outbreak of the winter or moose tick, Dermacentor albipictus, Pack. (Ixodoidea) in Saskatchewan. Bulletin of Entomological Research 17: 249-257.

CHOQUETTE, L.P.E., L.K. WHITTEN, G. RANKIN, AND C.M. SEAL. 1957. Notes on parasites found in reindeer (Rangifer tarandus) in Canada. Canadian Journal of Comparative Medicine 21: 199-203.

COOPER, K.W. 1946. The occurrence of the mite Cheyletiella parasitivorax (Megnin) in North America, with notes on its synonymy and "parasitic" habit. Journal of Parasitology 32: 480-482.

COWAN, I. MCT. 1943. Notes on the life history and morphology of Cephenomyia jellisoni Townsend and Lipoptena depressa Say, two dipterous parasites of the Columbian black-tailed deer (Odocoileus hemionus columbianus (Richardson)). Canadian Journal of Research (section D) 21: 171-187.

1944. Parasites, diseases and injuries of game animals in Banff, Jasper and Kootenay National Parks. Lands, Parks, and Forest Branch, National Parks Bureau. Pp. $1-42$.

1946. Parasites, diseases, injuries, and anomalies of the Columbian black-tailed deer, Odocoileus hemionus columbianus (Richardson), in British Columbia. Canadian Journal of Research (section D) 24: 71-103. 1955. An instance of scabies in the marten (Martes americana). Journal of Wildlife Management 19: 499.

DE VOS, A. 1957. Pregnancy and parasites of marten. Journal of Mammalogy 38: 412.

DODDS, D.G., A.M. MARTELL, AND R.E. YESCOTT. 1969. Ecology of the American dog tick, Dermacentor variabilis (Say), in Nova Scotia. Canadian Journal of Zoology 47: 171-181.

DREW, M.L., AND W.M. SAMUEL. 1985. Factors affecting transmission of larval winter ticks,

Dermacentor albipictus (Packard), to moose, Alces alces L. in Alberta, Canada. Journal of Wildlife Diseases 21: 274-282.

1986. Reproduction of the winter tick, Dermacentor albipictus, under field conditions in Alberta, Canada. Canadian Journal of Zoology 64: 714-721.

DYMOND, J.R. 1938. External parasites of bats. Canadian Entomologist 20: 20-21.

EIDMANN, H. 1935. Zur Kenntnis der Insektenfauna von Sud Labrador. Arbeiten Uber Morphologische und Taxonomische Entomologie 2: 81-105.

EMERSON, K.C. 1972. Checklist of the Mallophaga of North America (North of Mexico). Deseret Test Center. Dugway, Utah.

ERICKSON, A.B., AND P.R. HIGHBY. 1942. Parasites of the woodland caribou. Journal of Parasitology 28: 423.

EWING, H.E., AND E.W. BAKER. 1947. Myonyssus jamesoni, a new liponyssid mite (Acarina: Laelaptidae) from Blarina brevicauda (Say). Journal of Parasitology 33: 376-379.

FAIN, A. 1970. Redescription du type de Listrophorus validus Banks, 1910 (Acarina: Sarcoptiformes). Revue de Zoologie et de Botanique Africaines 81: 125-132.

FAIN, A., AND K. HYLAND. 1974. The listrophoroid mites in North America. II. The family Listrophoridae Megnin and Trovessart (Acarina: Sarcoptiformes). Bulletin Institut Royal des Sciences Naturelles de Belgique. Entomologie 50: 1-69.

FAIN, A., AND J.O. WHITAKER, JR. 1976. Dermacarus jonesi sp. n. (Acari Labidophorinae: Glycyphagidae) from Peromyscus maniculatus from western North America. Journal of Parasitology 62: 119-120.

FERRIS, G.F., AND F.R. COLE. 1922. A contribution to the knowledge of the Hippoboscidae (Diptera Pupipara). Parasitology 14: 178-205.

FIRLOTTE, W.R. 1948. A survey of the parasites of the brown Norway rat. Canadian Jounal of Comparative Medicine 12: 187-191.

FLOOK, D.R., AND J.E. STENTON. 1969. Incidence and abundance of certain parasites in wapiti in the national parks of the Canadian Rockies. Canadian Journal of Zoology 47: 795-803.

FULLER, H.S. 1943. Studies on Siphonaptera of eastern North America. Bulletin of the Brooklyn Entomological Society 38 : 18-23.

FYVIE, A. 1966. A review and current survey of the common diseases and parasites of wildlife in Ontario. Ontario Department of Lands and Forests Section Report (Wildlife) 63: 1-21.

GABBUTT, P.D. 1966. The distribution of some small mammals and their associated fleas from central Labrador. Ecology 42: 518-525.

GARVIE, M.B., J.A. MCKIEL, D.E. SONENSHINE, AND A. CAMPBELL. 1978. Seasonal dynamics of American dog tick, Dermacentor variabilis (Say), populations in southwestern Nova Scotia. Canadian Journal of Zoology 56: 28-39.

GIBBONS, R.J. 1939. Survey of Rocky Mountain spotted fever and sylvatic plague in western Canada during 1938. Canadian Public Health Journal 30: 184-187.

GIBBONS, R.J., AND F.A. HUMPHREYS. 1941. Plague surveys in western Canada. Canadian Public Health Journal 32: 24-28.

GIBBS, H.C. 1960. Disease investigation of barren-ground caribou. Wildlife Management Bulletin 15: 119-135.

GREGSON, J.D. 1941a. Host poisoning by Ixodes californicus Banks. Proceedings of the Entomological Society of British Columbia 37: 5-6.

1941b. Two new species of ticks from British Columbia (Ixodidae). Canadian Entomologist 73: 220-228.

$1941 \mathrm{c}$. Host immunity to ticks (Acarina). Proceedings of 
the Entomological Society of British Columbia 37: 12-13.

1942. The coast tick (Ixodes californicus Banks) problem in British Columbia. The Canadian

Entomologist 74: 3-5.

1953. Records of the tick Otobius megnini (Duges) from British Columbia (Acarina: Argasidae). Proceedings of the Entomological Society of British Columbia 49: 30.

1958. Host susceptibility to paralysis by the tick Dermacentor andersoni Stiles (Acarina: Ixodidae). Canadian Entomologist 90: 421-424.

GREGSON, J.D., AND G.M. KOHLS. 1952. The male of Ixodes soricis Gregson (Acarina: Ixodidae). Canadian Entomologist 84: 185-188.

GYORKOS, T.W., AND D.F.J. HILTON. 1982. Range extensions for some ectoparasites from rodents of southeastern Quebec. Canadian Journal of Zoology 60: 486-488.

HADWEN, S. 1913. On "tick paralysis" in sheep and man following bites of Dermacentor venustus, with notes on the biology of the tick. Parasitology 6: 283-297.

HALL, R.E. 1981. The Mammals of North America. 2nd ed. John Wiley and Sons. New York.

HARPER, F. 1956. The mammals of Keewatin. University of Kansas Miscellaneous Publications 12: 1-94.

1961. Land and fresh water mammals of the Ungava Peninsula. University of Kansas Miscellaneous Publications 27: 1-178.

HEARLE, E. 1938. The ticks of British Columbia. Scientific Agriculture 18: 341-354.

HEWITT, C.G. 1915. A contribution to a knowlege of Canadian ticks. Transactions of the Royal Society of Canada 9: 225-239.

HILTON, D.F.J., AND J.L. MAHRT. 1971.

Ectoparasites from three species of Spermophilus (Rodentia: Sciuridae) in Alberta. Canadian Journal of Zoology 49: 1501-1504.

HOLLAND, G.P. 1938. Phenomenal infestation of ectoparasites on marmot, weasel and packrat. Proceedings of the Entomological Society of British Columbia 35: 27-28.

1939. Notes on the ecology of Dermacentor andersoni in southern Alberta. Proceedings of the Entomological Society of British Columbia 36: 8-11.

1940. New records of Siphonaptera for British

Columbia. Proceedings of the Entomological Society of British Columbia 36: 11-12.

1941a. Further records of Siphonaptera for British Columbia. Proceedings of the Entomological Society of British Columbia 37: 10-14.

1941b. A survey of the rat fleas of the southern British Columbia coast with relation to plague studies.

Proceedings of the Entomological Society of British Columbia 37: 1-5.

1944. The distribution of some plague-important rodents and fleas in western Canada. Proceedings of the Entomological Society of British Columbia 41: 5-12.

1949. The Siphonaptera of Canada. Canadian Department of Agriculture Technical Bulletin 70: 1-306.

1950. Notes on Megabothris asio (Baker) and M. calcarifer (Wagner) with the description of a new subspecies (Siphonaptera: Ceratophyllidae). Canadian Entomologist 82: 126-133.

1952a. Notes on some Siphonaptera from Canada. Canadian Entomologist 84: 65-73.

1952b. Descriptions of fleas from Northern Canada (Siphonaptera). Canadian Entomologist 84: 297-308.

1979. Three new species of fleas from Canada (Siphonaptera). Canadian Entomologist 111: 713-719.

1984. The Fleas of Canada, Alaska, and Greenland (Siphonaptera). Memoirs of the Entomological Society of Canada No. 130

HOPKINS, G.H.E. 1954. Notes on some Mallophaga from bears. The Entomologist (London) 87: 140-146.

1960. Notes on some Mallophaga from mammals. Bulletin of the British Museum of Natural History (Entomology) 10: 77-98.

HOPPING, G.R. 1949. Parasitic Coleoptera. Proceedings of the Entomological Society of British Columbia 45: 16-17.

HORN, G.H. 1872. Descriptions of some new North American Coleoptera. Transactions of the American Entomological Society 4: 143-152.

HUBBARD, C.A. 1949a. Fleas in the collection of the Royal Ontario Museum of Zoology. Canadian Entomologist 81: 11-12.

1949b. A pictorial review of the North American chipmunk fleas. Entomological News 60: 253-261

HUNTER, D.M., AND J.M. WEBSTER. 1974. Effects of cuterebrid larval parasitism on deer-mouse metabolism. Canadian Journal of Zoology 52: 209-217.

HUNTER, D.M., R.M.F.S. SADLEIR, AND J.M. WEBSTER. 1972. Studies on the ecology of cuterebrid parasitism in deermice. Canadian Journal of Zoology 50: 25-29.

JAMESON, E.W., JR. 1943. Notes on the habits and siphonapterous parasites of the mammals of Welland County, Ontario. Journal of Mammalogy 24: 194-197. 1948. Myobiid mites (Acarina: Myobiinae) from shrews (Mammalia: Soricidae) of eastern North America. Journal of Parasitology 34: 336-342.

1950. The external parasites of the short-tailed shrew, Blarina brevicauda (Say). Journal of Mammalogy 31: 138-145.

JARVIS, T.D. 1910. The Acarina, with a host index to the species found in Ontario. Entomological Society of Ontario Annual Report 40: 82-109.

JELLISON, W.L. 1945. Siphonaptera: the genus Oropsylla in North America. Journal of Parasitology 31: 83-97.

JONES, G.S., AND H.H. THOMAS. 1980. Ticks from 
mammals from Prince Edward Island, New Brunswick, northern Nova Scotia, and Gaspe Peninsula, Quebec.

Canadian Journal of Zoology 58: 1394-1397.

1982. Mites, ticks, and fleas of the mice Zapus hudsonius and Napaeozapus insignis from the Maritime Provinces and Gaspe Peninsula, Quebec, Canada. Canadian Entomologist 114: 1031-1035.

1983. Distribution and ectoparasites of little brown bats, Myotis lucifugus, on Prince Edward Island. Canadian

Field-Naturalist 97: 320-321.

JORDAN, K. 1929. Notes on North American fleas.

Novitates Zoologicae 35: 28-29.

1932. On some nearctic Siphonaptera. Novitates

Zoologicae 41: 119-124.

JUDD, W.W. 1950. Mammal host records of Acarina and Insecta from the vicinity of Hamilton, Ontario. Journal of Mammalogy 31: 357-358.

1953. Mammal host records of Acarina and Insecta from the vicinity of London, Ontario. Journal of Mammalogy 34: 137-139.

1954a. Some records of ectoparasitic Acarina and Insecta from mammals in Ontario. Journal of Parasitology 40: 483-484.

1954b. A warble fly, Cuterebra sp., reared from a house mouse, Mus musculus, in the vicinity of London, Ontario. Canadian Field-Naturalist 68: 140.

1955. A collection of fleas from the vicinity of Fort Simpson, Northwest Territories, Canada. Journal of Parasitology 41: 441-442.

KELSALL, J.P. 1975. Warble fly distribution among some Canadian caribou. Proceedings of the First International Reindeer and Caribou Symposium. August 9-11, 1972. Fairbanks, Alaska.

KERR, G.R., AND J.C. HOLMES. 1966. Parasites of mountain goats in west central Alberta. Journal of Wildlife Management 30: 786-790.

KNIGHT, I.W.M. 1951. A report on mites infesting the muskrat (Ondatra zibethica osoyoosensis) in British Columbia. Canadian Entomologist 83: 279-280.

KO, R.C. 1972a. Biology of Ixodes cookei Packard (Ixodidae) of groundhogs (Marmota monax Erxleben). Canadian Journal of Zoology 50: 433-436.

1972b. The transmission of Ackertia marmotae Webster, 1967 (Nematoda: Onchocercidae) of groundhogs (Marmota monax) by Ixodes cookei. Canadian Journal of Zoology 50: 437-450.

1973. The larva of Ixodes cookei (Acarina: Ixodidae). Canadian Entomologist 105: 245-248.

KOK, N.J.J., F.S. LUKOSCHUS, AND F.V. CLULOW. 1970. Psorobia castoris spec. nov. (Acarina:

Psorergatidae), a new itch mite from the beaver, Castor canadensis. Canadian Journal of Zoology 48: 1419-1423. 1971. Three new itch mites from Canadian small mammals (Acarina: Psorergatidae). Canadian Journal of Zoology 49: 1239-1248.

KRANTZ, G.W. 1978. A manual of Acarology. 2nd ed.
Oregon State University Book Store Inc. Corvallis, Oregon.

LAWRENCE, R.F. 1948. Studies on some parasitic mites from Canada and South Africa. Journal of Parasitology 34: 364-379.

MACNAY, C.G. 1952. Summary of the more important insect infestations and occurrences in Canada in 1951. Annual Report of the Entomological Society of Ontario 82: 91-115.

1958. Summary of important insect infestations, occurrences and damage in Canada in 1957. Annual Report of the Entomological Society of Ontario 88: 63-78.

MAHRT, J.L., AND S.J. CHAI. 1972. Parasites of red squirrels in Alberta, Canada. Journal of Parasitology 58: 639-640.

MARGOLIS, L., AND J.R. ARTHUR. 1979. Synopsis of the Parasites of Fishes of Canada. Bulletin of the Fisheries Research Board of Canada No. 199. Department of Fisheries and Oceans. Ottawa.

MARTELL, A.M., R.E. YESCOTT, AND D.G. DODDS. 1969. Some records for Ixodidae of Nova Scotia. Canadian Journal of Zoology 47: 183-184.

MCALPINE, J.F. 1965. Insects and related terrestrial invertebrates of Ellef Ringnes Island. Arctic 18: 73-103.

MCDANIEL, B., J.P. SHOEMAKER, AND S.J. JOY. 1967. The discovery of Listrophorus leuckarti Pagenstecher on Microtus pennsylvanicus pennsylvanicus from North America. Proceedings of the Entomological Society of Washington 69: 340-343.

MCKENZIE, C.E., AND H.E. WELCH. 1979. Parasite fauna of the muskrat, Ondatra zibethica (Linnaeus, 1766), in Manitoba, Canada. Canadian Journal of Zoology 57: 640-646.

MCKIEL, J.A., R.R. HALL, AND G. COUSINEAU. 1960. Distribution of Xenopsylla cheopis (Rothsch.) (Siphonaptera: Pulicidae) in Canada. Canadian Entomologist 92: 428-429.

MCLEOD, J.A. 1933. A parasitological survey of the genus Citellus in Manitoba. Canadian Journal of Research (section D) 9: 108-127.

MITCHELL, C.J., AND R. BEHIN. 1965. Notes on some small mammal ectoparasites from Northwest Territories, Canada (Anoplura, Siphonaptera, and Acarina). Proceedings of the Entomological Society of Washington 67: 112.

MORRISON, F.O. 1937. Myiasis in the foot of a rat by Wohlfahrtia meigenii Schiner (Diptera, Metopiidae). Canadian Entomologist 69: 266-269.

NUTTALL, G.H.F. 1916. Notes on ticks. IV. Relating to the genus Ixodes and including a description of three new species and two new varieties. Parasitology 8 : 294-337.

PARKS, J.J., AND J.W. BARNES. 1955. Notes on the family Leptinidae including a new record of Leptinillus validus (Horn) in North America (Coleoptera). Annals 
of the Entomological Society of America 48: 417-421.

PETERSON, R.L. 1955. North American moose. University of Toronto Press, Toronto.

RADFORD, C.D. 1955. Some new and little-known mites of the genus Myocoptes Claparede (Acarina:

Listrophoridae). Parasitology 45: 275-286.

RICH, G.B. 1957. The ear tick, Otobius megnini (Dugs) (Acarina: Argasidae), and its record in British Columbia. Canadian Journal of Comparative Medicine and Veterinary Journal 21: 415-418.

RITCEY, R.W., AND R.Y. EDWARDS. 1958. Parasites and diseases of the Wells Gray moose herd. Journal of Mammalogy 39: 139-145.

ROBERT, A. 1962. Siphonapteres recoletes sur les petits rongeurs du Parc du Mont Tremblant et leurs relations avec leurs hutes. Annals of the Entomological Society of Quebec 7: 3-18.

ROTHSCHILD, N.C. 1902. Some new Nearctic fleas. Entomologists Record and Journal of Variation 14: $62-63$.

1905. On North American Ceratophyllus, a genus of Siphonaptera. Novitates Zoologicae 12: 153-174.

1906. Three new Canadian fleas. Canadian Entomologist 38: 321-325.

SAMUEL, W.M. 1981. Attempted experimental transfer of sarcoptic mange (Sarcoptes scabiei, Acarina: Sarcoptidae) among red fox, coyote, wolf and dog. Journal of Wildlife Disease 17: 343-346.

SAMUEL, W.M., AND M.J. BARKER. 1979. The winter tick, Dermacentor albipictus (Packard, 1869) on moose, Alces alces (L.), of central Alberta. Proceedings of the North American Moose Conference Workshop 15: 303-348.

SAMUEL, W.M., E.R. GRINNELL, AND A.J. KENNEDY. 1980. Ectoparasites (Mallophaga, Anoplura, Acari) on mule deer, Odocoileus hemionus, and white-tailed deer, Odocoileus virginianus, of Alberta, Canada. Journal of Medical Entomology 17: 15-17.

SAMUEL, W.M., W.K. HALL, J.G. STELFOX, AND W.D. WISHART. 1978. Parasites of mountain goat, Oreamnos americanus (Blainville), of west central Alberta with a comparison of the helminths of mountain goat and Rocky Mountain bighorn sheep, Ovis c. canadensis Shaw. Proceedings of the First International Mountain Goat Symposium. pp. 212-225.

SCHOLTEN, T.H., K. RONALD, AND D.M. MCLEAN. 1962. Parasite fauna of the Manitoulin Island region. I. Arthropoda parasitica. Canadian Journal of Zoology 40: 605-606.

SEALANDER, J.A. 1961. Haematological values of deer mice in relation to botfly infection. Journal of Mammalogy 42: 57-60.

SHEMANCHUK, J.A., AND J.W. KICENIUK. 1970. Ixodes spinipalpis Hadwen and Nuttall (Ixodidae) from southern Alberta. Canadian Journal of Zoology 48: 1142-1143.
SILLMAN, E.I. 1956. Studies on the biology of a cuterebrid (Cuterebridae: Diptera) infesting Peromyscus leucopus noveboracensis, Fischer, the white-footed mouse, in southern Ontario. Annual Report of the Entomological Society of Ontario (1955) 86: 89-97.

1957. Further laboratory and field observations on the ecology of some Ontario Cuterebridae (Diptera), in particular, Cuterebra angustifrons Dalmat, 1942. Entomological Society of Ontario Annual Report 87: 28-40.

SILLMAN, E.I., AND M.V. SMITH. 1959. Experimental infestation of Peromyscus leucopus with larvae of Cuterebra angustifrons. Science 130: 165-166.

SMITH, H.C. 1981. First record for Canada of the bat mite Spinturnix globosus and a new host, Myotis lucifugus. Canadian Field-Naturalist 95: 206-207.

SPENCER, G.J. 1934. The bedbugs of British Columbia. Proceedings of the Entomological Society of British Columbia 30: 43-45.

1937. The menace of rat parasites in Vancouver in 1936. Proceedings of the Entomological Society of British Columbia 33: 44-45.

1938a. Ectoparasites of deer in British Columbia. Proceedings of the Entomological Society of British Columbia 34: 15-19.

1938b. Ectoparasites of birds and mammals of British Columbia. II. A preliminary list of the Pupipara, louse flies. Proceedings of the Entomological Society of British Columbia 34: 39-45.

1939a. Ectoparasites of birds and mammals of British Columbia. IV. The parasites of bats. Proceedings of the Entomological Society of British Columbia 36: 16-18.

1939b. Ectoparasites of birds and mammals of British Columbia. V. Parasites of Domestic Animals (Mammals). Proceedings of the Entomological Society of British Columbia 36: 19-23.

1940. Ectoparasites of birds and mammals in British Columbia. VI. A preliminary list of parasitic mites. Proceedings of the Entomological Society of British Columbia 36: 14-18.

1956. Some records of ectoparasites from flying squirrels. Proceedings of the Entomological Society of British Columbia 52: 32-34.

1957. North American beetles infesting mammals. Proceedings of the Entomological Society of British Columbia 53: 21-22.

1966. Anoplura from British Columbia and some adjacent areas. Journal of the Entomological Society of British Columbia 63: 23-30.

STELFOX, J.G. 1962. Liver, lungs and larvae. Parasites and disease in moose, deer and elk in Alberta. Alberta Landa, Forests, and Wildlife Annual Report 5: 5-12.

STRICKLAND, E.H. 1938. An annotated list of the Diptera (flies) of Alberta. Canadian Journal of Research (section D) 16: 175-219. 
SWEATMAN, G.K. 1958a. Redescription of Chorioptes texanus, a parasitic mite from the ears of reindeer in the Canadian arctic. Canadian Journal of Zoology 36: 525-528.

1958b. The biology of Otodectes cynotis, the ear canker mite of carnivores. Canadian Journal of Zoology 36: 849-862.

THOMPSON, G.B. 1934. Records of Siphunculata and Mallophaga from Canadian hosts. Canadian Entomologist 66: 279-281.

TIMMERMANN, H.R., AND M.W. LANKESTER. 1980. Studies of winter tick, Dermacentor albipictus, on the bell of moose in northwestern Ontario. Proceedings of the North American Moose Conference Workshop 16: $137-151$.

TYRRELL, J.B. 1883. On the occurrence in Canada of two species of parasitic mites. Proceedings of the Canadian Institute 1: 332-342.

VAN ZYLL DE JONG, C.G. 1966. Parasites of the Canada lynx, Felis ( Lynx) canadensis (Kerr). Canadian Journal of Zoology 44: 499-509.

WAGNER, J.N. 1933. Aphanipteren-material aus der sammlung des Zoologischen Museum der Berliner Universitat. Mitteilungen aus dem Zoolog. Museum in Berlin 18: 338-362.

1936a. The fleas of British Columbia. Canadian Entomologist 68: 193-201.

1936b. Neue nordamerikanische Floharten. Zeitschrift fur Parasitenkunde 8: 543-658.

WALKER, M.L., AND W.W. BECKLUND. 1970. Checklist of the internal and external parasites of deer, Odocoileus hemionus and $O$. virginianus, in the United States and Canada. Special Publication 1. IndexCatalogue of Medical and Veterinary Zoology, National Animal Parasite Laboratory, Veterinary Sciences Research Division, Agricultural Research Services pp. $1-45$.

WATSON, T.G., AND R.C. ANDERSON. 1975. Seasonal changes in louse populations on white-tailed deer (Odocoileus virginianus). Canadian Journal of Zoology 53: 1047-1054.

1976. Ixodes scapularis Say on white-tailed deer (Odocoileus virginianus) from Long Point, Ontario. Journal of Wildlife Diseases 12: 66-71.
WEBSTER, W.A. 1966. Ixodes texanus in eastern Canada. Bulletin of the Wildlife Disease Association 2: 78-79.

WEBSTER, W.A., AND R.C. STEWART. 1964. Tricholipeurus lipeuroides (Mgnin) (Mallophaga) from a white-tailed deer in Quebec. Canadian Journal of Zoology 42: 323.

WHITAKER, J.O. JR., AND T.W. FRENCH. 1982. Ectoparasites and other associates of some insectivores and rodents from New Brunswick. Canadian Journal of Zoology 60: 2787-2797.

WHITAKER, J.O. JR., AND N. WILSON. 1974. Host and distribution lists of mites (Acari), parasitic and phoretic, in the hair of wild mammals of North America, north of Mexico. The American Midland Naturalist 91: $1-67$.

WHITEHEAD, W.E. 1934. Records of some Quebec Mallophaga and Anoplura. Report of the Quebec Society for the Protection of Plants 26: 84-87.

WILKINSON, P.R. 1965. A first record of paralysis of a deer by Dermacentor andersoni (Stiles) and notes on the "host-potential" of deer in British Columbia.

Proceedings of the Entomological Society of British Columbia 62: 28-30.

1970. Dermacentor ticks on wildlife and new records of paralysis. Journal of the Entomological Society of British Columbia 67: 24-29.

WILKINSON, P.R., R. FYFE, AND J.E.H. MARTIN. 1980. Further records of Ornithodoros ticks on prairie falcons and in bat-inhabited buildings in Canada. Canadian Field-Naturalist 94: 191-193.

WILSON, N. 1967. Ectoparasites of Canadian birds and mammals. Proceedings of the Entomological Society of Washington 69: 349-353.

WRIGHT, B. 1979. Mites, ticks, fleas, and lice in the Nova Scotia Museum and Acadia University Museum Collections. Proceedings of the Nova Scotia Institute of Science 29: 185-196. 


\section{SUPPLEMENTARY REFERENCES}

ANDERSON, R.C. 1962. The helminth and arthropod parasites of the white-tailed deer (Odocoileus virginianus): a general review.

ANDERSON, R.C., AND M.W. LANKESTER. 1974. Infectious and parasitic diseases and arthropod pests of moose in North America. Nature Canada (Quebec) 101: 23-50.

BAKER, C.F. 1895. Preliminary studies in Siphonaptera VII. The Canadian Entomologist 27: 221-222.

1904. A revision of American Siphonaptera, or fleas, together with a complete list and bibliography of the group. Proceedings of the United States National Museum 27: 365-469.

BAKER, E.W., T.M. EVANS, D.J. GOULD, W.B. HULL, AND H.L. KEEGAN. 1956. A manual of parasitic mites of medical or economic importance. Technical Publication of the National Pest Control Association. New York. See pages 96-97.

BAILEY, V. 1923. The combing claws of the beaver. Journal of Mammalogy 4: 77-79.

BANKS, N. 1908. A revision of the Ixodoidea, or ticks, of the United States. United States Department of Agriculture, Bureau of Entomology, Technical Series 15. See pages 28, 29, and 32 .

BECKLUND, W.W., AND C.M. SENGER. 1967. Parasites of Ovis canadensis canadensis in Montana, with a checklist of the internal and external parasites of the Rocky Mountain bighorn sheep in North America. Journal of Parasitology 53: 157-165.

BISHOP, F.C., AND H.L. TREMBLEY. 1945. Distribution and hosts of certain American ticks. Journal of Parasitology 31: 1-54.

BISHOPP, F.C. AND H.P. WOOD. 1913. The biology of some North American ticks of the genus Dermacentor. Parasitology 6: 153-187.

BROWN, J.H. 1944. The spotted fever and other Albertan ticks. Canadian Journal of Research (section D) 22: 36-51.

COOLEY, R.A., AND G.M. KOHLS. 1945. The genus Ixodes in North America. National Institute of Health Bulletin 184: 1-246.

COWAN, I. MCT. 1951. The diseases and parasites of big game mammals of western Canada. Proceedings of the 5th Annual Game Convention (1951): 37-64.

DREW, M.L., W.M. SAMUEL, G.M. LUKIWSKI, AND J.N. WILLMAN. 1985. An evaluation of burning for control of winter ticks, Dermacentor albipictus, in central Alberta. Journal of Wildlife Diseases 21: 313-315.

EADS, R.B., AND E.G. CAMPOS. 1984. Notes on the deer keds Neolipoptena ferrisi and Lipoptena depressa (Diptera: Hippoboscidae) from Colorado, U.S.A. Journal of Medical Entomology 21: 245.
EADS, R.B., B.C. NELSON, G.O. MAUPIN, AND A.M. BARNES. 1985. Orchopeas fleas (Siphonaptera: Ceratophyllidae) of the western gray squirrel, Sciurus griseus. Journal of Medical Entomology 22: 630-636.

FAIN, A., N.J.J. KOK, F.S. LUKOSCHUS, AND F.V. CLULOW. 1971. Notes on the hypopial nymphs phoretic on mammals in Canada with description of a new species (Acarina: Sarcoptiformes). Canadian Journal of Zoology 49: 15-18.

FAIN, A., AND J.O. WHITAKER, JR. 1973. Phoretic hypopi of North American mammals (Acarina: Sarcoptiformes, Glycyphagidae). Acarologia 15: 144-170.

FERRIS, G.F. 1951. The Sucking Lice. Memoirs of the Pacific Coast Entomological Society. Volume 1. California Academy of Sciences, San Francisco, California. 320pp.

GOLINI, V.I., S.M. SMITH, AND D.M. DAVIES. 1968. Probable larviposition by Cephenomyia phobifer (Clark) (Diptera: Oestridae). Canadian Journal of Zoology 46: 809-814.

HARPER, A.M. 1979. A bibliography of Alberta entomology. Alberta Agriculture Publication. Alberta, Canada $101 \mathrm{pp}$.

HERRIN, C.S. 1970. A systematic revision of the genus Hirstionyssus (Acari: Mesostigmata) of the nearctic region. Journal of Medical Entomology 7: 391-437.

HOLLAND, G.P. 1949. A revised check list of the fleas of British Columbia. Proceedings of the Entomological Society of British Columbia 45: 7-14.

1950. Notes on some British Columbian fleas, with remarks on their relationship and distribution. Proceedings of the Entomological Society of British Columbia 46: 5-13.

HOOKER, W.M. 1909. The geographical distribution of American ticks. Journal of Economic Entomology 2: 403-428.

HORN, G.H. 1882. Notes on some little known genara and species of Coleoptera. Transactions of the American Entomological Society 10: 113-126.

JOHNSTON, D.E. 1959. Some new synonymy in the Haemogamasidae, Laelaptidae and Diplogyniidae indicated by an examination of Banks' types of Mesostigmata (Acarina). Psyche 66: 60-62.

JOHNSTON, D.E., AND D.L. DEGIUSTI. 1961. Ecological and systematic notes on some chigger-mites of the genera Gahrliepia, Trombicula, and

Euschongastia (Acarina: Trombiculidae) in Michigan and Ontario. Journal of Parasitology 47: 11-12.

JOHNSTON, P.T., AND R. TRAUB. 1954. Revision of the flea genus Peromyscopsylla. Smithsonian Miscellaneous Collection 123: 1-68.

KELLOGG, V.L., AND G.F. FERRIS. 1915. The Anoplura and Mallophaga of North American Mammals. Stanford University Press. California. 74pp. +8 plates. 
LANKESTER, M.W. 1985. Pests, parasites and diseases of moose (Alces alces) in North America. In Press.

MAIL, G.A., AND J.D. GREGSON. 1938. Tick paralysis in British Columbia. Canadian Medical Association Journal: 532-537.

NUTTALL, G.H.F., AND C. WARBURTON. 1908. Ticks. A monograph of the Ixodidae. Part 1. Argasidae. pp. 1-104.

1911. Ticks. A monograph of the Ixodidae. Part II. Ixodidae. pp. 105-348.

RADOVSKY, F.J. 1960. Haemogamasus liponyssoides hesperus, n. ssp., with a discussion of the $H$. liponyssoides complex (Acarina: Haemogamasidae). Journal of Parasitology 46: 401-409.

SPENCER, G.J. 1936. A check list of the fleas of British Columbia, with a note on fleas in relation to sawdust in homes. Proceedings of the Entomological Society of British Columbia 32: 11-17.
SPENCER, J.D. 1938. Further notes on the fleas of British Columbia. Proceedings of the Entomological Society of British Columbia 35: 36-38.

WILKINSON, P.R. 1964. Factors affecting distribution of the Rocky Mountain wood tick (Dermacentor andersoni Stiles) in British Columbia. Bulletin of the Ecological Society of America 45: 53.

1967. The distribution of Dermacentor ticks in Canada in relation to bioclimatic zones. Canadian Journal of Zoology 45: 517-537.

1968. Phenology, behaviour, and host-relations of Dermacentor andersoni Stiles in outdoor "rodentaria", and in nature. Canadian Journal of Zoology 46: 677-689.

WILKINSON, P.R., H.R. ABBOTT, AND J.N.

WILLMAN. 1982. Location of larvae of the winter tick, Dermacentor albipictus (Pack.), in Elk Island National Park, Alberta. Canadian Field-Naturalist 96: 211-213. 
Acari 1

Acaridida 1

Actinedida 3

Allothrombium spp. 6

Amalaraeus athabascae $\quad 17$

Amalaraeus dissimilis $\quad 17$

Amorphacarus hengererorum 3

Amphalius necopinus

see Amphalius runatus necopinus

Amphalius runatus necopinus 17

Amphipsylla sibirica pollionis $\quad 38$

Amphipsylla washingtona

39

Androlaelaps casalis 6

Androlaelaps fahrenholzi 6

Androlaelaps glasgowi

see Androlaelaps fahrenholzi

Androlaelaps spp. $\quad 7$

Anomiopsyllus montanus

29

Arachnida 1

Arctopsylla ursi

see Chaetopsylla tuberculaticeps

Argasidae 9

Atheopsylla septentrionalis

see Oropsylla arctomys

Atricholaelaps glasgowi

see Androlaelaps fahrenholzi

Atyphloceras artius

see Atyphloceras multidentatus

multidentatus 29

Atyphloceras bishopi 29

Atyphloceras multidentatus multidentatus

Bakerdania spp. 4

Basilia forcipata 15

Basilia spp. $\quad 15$

Blarinobia simplex 3

Calliphoridae 14

Callistopsyllus campestris

see Callistopsyllus terinus campestris

29

Callistopsyllus terinus campestris

Callistopsyllus terinus terinus

Caratonyssus occidentalis see Steatonyssus occidentalis

9

Catallagia borealis $\quad 30$

Catallagia chamberlini see Catallagia sculleni sculleni

Catallagia charlottensis $\quad 30$

Catallagia dacenkoi fulleri

Catallagia decipiens 30

Catallagia jellisoni 31

Catallagia mathesoni 31

Catallagia sculleni chamberlini

Catallagia sculleni sculleni

Catallagia spp. 31

Cediopsylla inaequalis inaequalis

Cediopsylla simplex $\quad 40$

Cephenemyia apicata 15

Cephenemyia jellisoni $\quad 15$

Cephenemyia nasalis

see Cephenemyia trompe 15

Cephenemyia phobifer
Cephenemyia trompe

Cephenemyia spp. $\quad 15$

Ceratonyssus occidentalis see Liponyssus occidentalis

Ceratophyllidae 17

Ceratophyllus abantis see Megabothris abantis

Ceratophyllus acamantis see Thrassis acamantis

Ceratophyllus acerbus see Megabothris acerbus

Ceratophyllus adustus $\quad 17$

Ceratophyllus agilis see Orchopeas sexdentatus agilis

Ceratophyllus alaskensis see Oropsylla alaskensis $\quad 27$

Ceratophyllus arctomys see Oropsylla arctomys

Ceratophyllus asio see Megabothris asio

Ceratophyllus atrox see Megabothris atrox

Ceratophyllus bacchi see Thrassis bacchi bacchi

Ceratophyllus bakeri see Monopsyllus thambus

Ceratophyllus bruneri see Opisocrostis bruneri

Ceratophyllus caedens see Orchopeas caedens caedens

Ceratophyllus caedens durus see Orchopeas caedens durus

Ceratophyllus canadensis see Nosopsyllus fasciatus

Ceratophyllus charlottensis see Catallagia charlottensis

Ceratophyllus coloradensis see Tarsopsylla octodecimdentata coloradensis 28

Ceratophyllus diffinis

Ceratophyllus eumolpi see Monopsyllus eumolpi 22

Ceratophyllus eumolpi eumolpi see Monopsyllus eumolpi eumolpi

Ceratophyllus euphorbi see Malaraeus euphorbi

Ceratophyllus fasciatus see Nosopsyllus fasciatus

Ceratophyllus gallinae $\quad 17$

Ceratophyllus garei 18

Ceratophyllus idahoensis see Oropsylla idahoensis $\quad 28$

Ceratophyllus ignotus albertensis see Foxella ignotus albertensis

Ceratophyllus ignotus recula see Foxella ignota recula $\quad 18$

Ceratophyllus labis see Opisocrostis labis

Ceratophyllus leucopus see Orchopeas leucopus

Ceratophyllus lucifer see Megabothris lucifer 
Ceratophyllus lunatus tundrensis $\quad 18$

Ceratophyllus niger $\quad 18$

Ceratophyllus penicilliger see Amalaraeus dissimilis

Ceratophyllus petiolatus see Thrassis petiolatus 29

Ceratophyllus petrochelidoni 18

Ceratophyllus poeantis see Oropsylla idahoensis 28

Ceratophyllus pollionis see Amphipsylla sibirica pollionis

Ceratophyllus pseudarctomys see Opisodasys pseudarctomys

Ceratophyllus quirini see Megabothris quirini $\quad 21$

Ceratophyllus saundersi see Opisocrostis bruneri

Ceratophyllus telchinum see Malaraeus telchinus

Ceratophyllus telegoni see Delotelis telegoni 32

Ceratophyllus terinus see Callistopsyllus terinus terinus

Ceratophyllus thambus see Monopsyllus thambus 23

Ceratophyllus tuberculatus tuberculatus see Opisocrostis tuberculatus tuberculatus 24

Ceratophyllus vesperalis see Opisodasys vesperalis

Ceratophyllus vison see Monopsyllus vison

Ceratopsylla insignis see Myodopsylla insignis

Ceratopsylla palposus see Myodopsylla palposa 38

Cervophthirius ferrisi see Solenopotes ferris

Chaetopsylla lotoris

Chaetopsylla setosa

Chaetopsylla tuberculaticeps

Chelicerata 1

Cheyletidae 3

Cheyletiella parasitivorax

Chorioptes texanus 3

Cimicidae 15

Cimex pilosellus

Coleoptera 13

Comatacarus americanus 5

Conorhinopsylla stanfordi 31

Corrodopsylla curvata curvata

Corrodopsylla curvata obtusata

Corrodopsylla hamiltoni 32

Corypsylla jordani 32

Corypsylla kohlsi 32

Corypsylla ornata 32

Corypsylloides kohlsi see Corypsylla kohlsi

Ctenocephalides canis 40

Ctenocephalides felis

Ctenocephalides felis felis 41

Ctenophthalmus pseudagyrtes pseudagyrtes 32

Ctenophthalmus wenmanni see Epitedia wenmanni wenmanni
Ctenophyllus armatus

Ctenophyllus terribilis see Ctenophyllus armatus

Ctenopsyllus hamifer see Peromyscopsylla hamifer hamifer

Ctenopsyllus hygini see Nearctopsylla genalis hygini

Ctenopsyllus hyrtaci see Nearctopsylla hyrtaci 36

Ctenopsyllus ravalliensis see Peromyscopsylla ravalliensis

Ctenopsyllus rawailliensis see Peromyscopsylla ravalliensis

Ctenopsyllus segnis see Leptopsylla segnis

Ctenopsyllus selenis see Peromyscopsylla selenis

Cuterebra americana var. polita

Cuterebra angustifrons $\quad 14$

Cuterebra emasculator $\quad 14$

Cuterebra fasciata $\quad 14$

Cuterebra fontinella 14

Cuterebra grisea 14

Cuterebra similis $\quad 14$

Cuterebra tenebrosa

Cuterebra spp. $\quad 14$

Cuterebridae $\quad 14$

Cyrtolaelaps spp. 9

Dactylopsylla comis $\quad 18$

Dactylopsylla ignota albertensis see Foxella ignota albertensis

Dactylopsylla ignota recula see Foxella ignota recula

Dactylopsylla rara 18

Damalinia concavifrons $\quad 16$

Damalinia lipeuroides see Tricholipeurus lipeuroides

Damalinia longicornis 16

Damalinia oreamnidis $\quad 16$

Damalinia ovis 16

Damalinia sedecimdecembrii $\quad 16$

Dasypsyllus gallinulae perpinnatus

Delotelis hollandi 32

Delotelis telegoni 32

Dermacarus hylandi 1

Dermacarus hypudaei 1

Dermacarus hypudaei septentrionalis

Dermacarus jonesi 1

Dermacarus newyorkensis 1

Dermacarus ondatrae 1

Dermacarus sciurinus 1

Dermacarus validus 1

Dermacarus sp. 1

Dermacentor albipictus $\quad 10$

Dermacentor andersoni $\quad 10$

Dermacentor variabilis 11

Dermacentor venustus see Dermacentor andersoni

Dermanyssidae 6

Diamanus montanus see Thrassis spenceri spenceri 
Diptera $\quad 14$

Dolichopsyllus stylosus

Doratopsylla blarinae

Doratopsylla curvata see Corrodopsylla curvata curvata

Doratopsylla curvata obtusata see Corrodopsylla curvata obtusata

Doratopsylla hamiltoni see Corrodopsylla hamiltoni

Eadiea condylurae 3

Echinolaelaps echidninus 7

Echinonyssus blarinae 7

Echinonyssus isabellinus 7

Echinonyssus talpae 7

Echinonyssus spp. 7

Enderleinellus marmotae $\quad 42$

Enderleinellus nitzschi $\quad 42$

Enderleinellus suturalis $\quad 42$

Enderleinellus uncinatus see Microphthirus uncinatus

Epitedia faceta 34

Epitedia inopina see Neopsylla inopina 36

Epitedia scapani 33

Epitedia stewarti 33

Epitedia wenmanni wenmanni

Euhaemogamasus liponyssoides see Haemogamasus liponyssoides

Euhaemogamasus microti see Haemogamasus longitarsus

Euhoplopsyllus glacialis affinis

Euhoplopsyllus glacialis glacialis

Euhoplopsyllus glacialis lynx

Eulaelaps stabularis 7

Euryparasitus spp. $\quad 9$

Euschoengastia blarinae 5

Euschoengastia setosa 5

Euschoengastia spp. 5

Eutrichophilus setosus $\quad 16$

Eutrombicula harperi 5

Fahrenholzia pinnata 42

Felicola spenceri 16

Foxella ignota albertensis

Foxella ignota ignota $\quad 18$

Foxella ignota recula $\quad 18$

Gamasida 6

Gemasus spp.

Geomydoecus spp.

Gliricoptes zapus 2

Gliricoptes spp. 2

Glycyphagidae 1

Glycyphagidae gen. spp. 1

Glycyphagus hypudaei 1

Haemaphysalis cinnabarina 11

Haemaphysalis leporis-palustris

Haematopinidae 42

Haematopinus spinulosus see Polyplax spinulosa

Haemodipsus ventricosus

Haemodipsus spp.
Haemogamasus ambulans 7

Haemogamasus hirsutus 7

Haemogamasus liponyssoides 7

Haemogamasus longitarsus 8

Haemogamasus reidi 8

Haemogamasus spp. 8

Haemolaelaps glasgowi 8

Halarachnidae 6

Hemiptera 15

Hippoboscidae 14

Hirstionyssus isabellinus $\quad 8$

Hirstionyssus occidentalis 8

Hirstionyssus talpae $\quad 8$

Hirstionyssus spp. $\quad 8$

Hoplopleura acanthopus $\quad 42$

Hoplopleura arboricola $\quad 42$

Hoplopleura erratica $\quad 42$

Hoplopleura hesperomydis 42

Hoplopleura oenomydis 42

Hoplopleura sciuricola 42

Hoplopleura trispinosa $\quad 42$

Hoplopleuridae 42

Hoplopsyllus affinis

see Euhoplopsyllus glacialis affinis

Hoplopsyllus glacialis

see Euhoplopsyllus glacialis glacialis

Hoplopsyllus glacialis lynx

see Euhoplopsyllus glacialis lynx

41

Hyperlaelaps spp. $\quad 8$

Hypoaspis spp. $\quad 8$

Hystrichopsylla americana

see Stenoponia americana 38

Hystrichopsylla dippiei ssp. $\quad 34$

Hystrichopsylla dippiei dippiei 34

Hystrichopsylla dippiei spinata 34

Hystrichopsylla dippiei truncata 34

Hystrichopsylla gigas dippiei

see Hystrichopsylla occidentalis occidentalis 34

Hystrichopsylla occidentalis occidentalis

Hystrichopsylla schefferi 35

Hystrichopsylla spinata

see Hystrichopsylla dippiei spinata

Hystrichopsylla tahavuana 35

Hystrichopsyllidae 29

Insecta 13

Ixodes angustus 11

Ixodes californicus 11

Ixodes cookei 12

Ixodes dentatus spinipalpis

see Ixodes spinipalpus

Ixodes hexagonus var. cookei

see Ixodes cookei $\quad 12$

Ixodes hearlei 12

Ixodes hexagonus 12

Ixodes kingi 12

Ixodes marmotae 12

Ixodes marxi 12

Ixodes muris 12

Ixodes ochotonae $\quad 12$

Ixodes pacificus $\quad 12$

Ixodes ricinus $\quad 12$ 
Ixodes rugosus 13

Ixodes scapularis 13

Ixodes sculptus 13

Ixodes soricis 13

Ixodes spinipalpus 13

Ixodes texanus 13

Ixodes spp. 13

Ixodida 9

Ixodidae 10

Laelapidae 6

Laelaps alaskensis $\quad 8$

Laelaps clethrionomydis

Laelaps kochi 8

Laelaps microti see Laelaps kochi

Laelaps multispinosus

8

Laelaps muris 8

Laelaps pedalis see Eulaelaps stabularis

Laelaps propheticus 9

Laelaps spp. 9

Laelaspis spp. $\quad 9$

Leptinidae 13

Leptinillus aplodontiae

Leptinillus validus 13

Leptinus americanus

Leptinus aplodontiae see Leptinillus aplodontiae

Leptinus validus see Leptinillus validus

Leptopsylla segnis $\quad 39$

Leptopsyllidae $\quad 38$

Linognathidae 43

Linognathoides montanus

Linognathoides inornatus see Neohaematopinus inornatus 43

Linognathus ferrisi see Solenopotes ferrisi 43

Liponyssus montanus 6

Liponyssus occidentalis 6

Liponyssus spp. 6

Lipoptena depressa $\quad 14$

Lipoptena ferrisi see Neolipoptena ferrisi $\quad 15$

Listrophoridae 2

Listrophoridae gen. spp. 2

Listrophorus americanus 2

Listrophorus dicrostonyx 2

Listrophorus leuckarti 2

Listrophorus mexicanus 2

Listrophorus mexicanus squamiferus

Listrophorus mustelae

see Lynxacarus mustelae 2

Listrophorus ondatrae 2

Listrophorus phenacomys 2

Listrophorus synaptomys 2

Listrophorus validus 2

Listrophorus spp. 2

Lynxacarus mustelae 2

Macronyssidae 9

Macronyssus crosbyi
Malaraeus bitterrootensis

Malaraeus euphorbi 19

Malaraeus penicilliger athabascae see Amalaraeus athabascae

Malaraeus penicilliger dissimilis see Amalaraeus dissimilis

Malaraeus telchinus 19

Mallophaga 16

Megabothris abantis $\quad 19$

Megabothris acerbus $\quad 19$

Megabothris asio $\quad 20$

Megabothris asio asio $\quad 20$

Megabothris asio megacolpus

Megabothris atrox 20

Megabothris calcarifer gregsoni 20

Megabothris clantoni clantoni 21

Megabothris groenlandicus $\quad 21$

Megabothris immitis see Megabothris groenlandicus

Megabothris lucifer

21

Megabothris obscurus

see Megabothris clantoni clantoni

Megabothris quirin

Megarthroglossus divisus divisus

35

Megarthroglossus divisus exsecatus

Megarthroglossus longispinus

see Megarthroglossus divisus divisus 35

Megarthroglossus procus procus $\quad 35$

Megarthroglossus pygmaeus see Megarthroglossus spenceri

Megarthroglossus sicamus $\quad 35$

Megarthroglossus similis

see Megarthroglossus procus procus 35

Megarthroglossus spenceri 35

Melophagus ovinus montanus

14

Meringis parkeri $\quad 35$

Meringis shannoni 35

Microphthirus uncinatus 43

Miyatrombicula esoensis 5

Monopsyllus ciliatus protinus

Monopsyllus eumolpi 22

Monopsyllus eumolpi canadensis see Monopsyllus eumolpi eumolpi

Monopsyllus eumolpi eumolpi 22

Monopsyllus thambus 23

Monopsyllus tolli 23

Monopsyllus vison 23

Monopsyllus wagneri ophidius

Monopsyllus wagneri systaltus

Monopsyllus wagneri wagneri

Myobia caudata 3

Myobia musculi 3

Myobia simplex

see Blarinobia simplex

3

Myobia spp. 3

Myobiidae 3

Myocoptes canadensis

see Myocoptes japonensis canadensis

Myocoptes japonensis japonensis 2

Myocoptes squamosus 2

Myocoptes tenax see Trichoecius tenax 3 
Myocoptes spp. 2

Myocoptidae 2

Myodopsylla gentilis 38

Myodopsylla insignis $\quad 38$

Myodopsylla palposa 38

Myodopsylloides palposus

see Myodopsylla palposa

Myonyssus jamesoni 6

Nearctopsylla brooksi 35

Nearctopsylla genalis $\quad 36$

Nearctopsylla genalis genalis $\quad 36$

Nearctopsylla genalis hygini 36

Nearctopsylla grahami 36

Nearctopsylla hygini columbiana see Nearctopsylla jordani 36

Nearctopsylla hygini laurentina see Nearctopsylla genalis genalis 36

Nearctopsylla hyrtaci $\quad 36$

Nearctopsylla jordani 36

Nearctopsylla traubi 36

Neohaematopinus antennatus semifasciatus see Neohaematopinus sciurinus

Neohaematopinus inornatus 43

Neohaematopinus laeviusculus 43

Neohaematopinus marmotae

Neohaematopinus sciurinus

Neohaematopinus sciuropteri

Neohaematopinus semifasciatus see Neohaematopinus sciurinus

Neolipoptena ferrisi $\quad 15$

Neopsylla faceta see Epitedia faceta 33

Neopsylla inopina 36

Neopsylla scapani see Epitedia scapani

Neoschongastia blarinae 6

Neotrichodectes mephitidis $\quad 16$

Neotrombicula basignata 5

Neotrombicula harperi 5

Neotrombicula lipovskyi 5

Neotrombicula microti 6

Nosopsyllus fasciatus $\quad 24$

Nycteribiidae $\quad 15$

Nycteridopsylla vancouverensis

Odontopsyllus dentatus $\quad 39$

Odontopsyllus multispinosus

Oedemagena tarandi

15

Oestridae 15

Ologamasidae 9

Ololaelaps spp. $\quad 9$

Ondatralaelaps multispinosa see Laelaps multipinosus

Opisocrostis bruneri 24

Opisocrostis labis $\quad 24$

Opisocrostis tuberculatus tuberculatus

Opisodasys keeni keeni 25

Opisodasys pseudarctomys

Opisodasys vesperalis 25

Orchopeas caedens $\quad 25$

Orchopeas caedens caedens

Orchopeas caedens durus
Orchopeas howardi howardi

Orchopeas leucopus 26

Orchopeas nepos 27

Orchopeas sexdentatus agilis

Orchopeas wickhami

see Orchopeas howardi howardi

26

Ornithodoros kelleyi 9

Ornithodoros megnini see Otobius megnini $\quad 10$

Ornithodoros spp. $\quad 9$

Ornithonyssus bacoti 9

Oropsylla acamantis see Thrassis acamantis

Oropsylla alaskensis $\quad 27$

Oropsylla arctomys 27

Oropsylla bacchi see Thrassis bacchi bacchi

Oropsylla bruneri see Opisocrostis bruneri

Oropsylla idahoensis $\quad 28$

Oropsylla rupestris $\quad 28$

Oropsylla tuberculata tuberculata see Opisocrostis tuberculatus tuberculatus 24

Orycteroxenus canadensis 1

Orycteroxenus soricis 1

Otodectes cynotis 3

Otobius megnini 10

Parasitiformes 6

Paraspinturnix globosus see Spinturnix globosus 9

Peromyscopsylla catatina 39

Peromyscopsylla hamifer hamifer

Peromyscopsylla hamifer markworthi see Peromyscopsylla hamifer hamifer $\quad 39$

Peromyscopsylla hesperomys adelpha $\quad 40$

Peromyscopsylla hesperomys hesperomys 40

Peromyscopsylla hesperomys pacifica $\quad 40$

Peromyscopsylla ostsibirica longiloba $\quad 40$

Peromyscopsylla ravalliensis $\quad 40$

Peromyscopsylla scotti $\quad 40$

Peromyscopsylla selenis $\quad 40$

Phormia regina 14

Platypsyllus castoris $\quad 14$

Pneumonyssus spp. 6

Polyplax alaskensis 43

Polyplax auricularis $\quad 43$

Polyplax spinulosa 43

Prolabidocarpus canadensis 2

Protomyobia americana 3

Protomyobia brevisetosa 3

Protomyobia claparedei 3

Psorergates canadensis 4

Psorergates cinereus 4

Psorergates simplex 4

Psorergates watsoni 4

Psorergates spp. 4

Psorergatidae 4

Psorobia castoris 4

Psoroptidae 3

Psoroptes ovis 3

Pulex bruneri see Opisocrostis bruneri 
Pulex charlottensis see Catallagia charlottensis 30

Pulex coloradensis see Tarsopsylla octodecimdentata coloradensis 28

Pulex fasciatus see Nosopsyllus fasciatus

Pulex irritans 41

Pulex keeni see Opisodasys keeni keeni

Pulex longispinus see Megarthroglossus divisus divisus

Pulex ursi see Chaetopsylla tuberculaticeps

Pulicidae $\quad 40$

Pygmephoridae 4

Pygmephorus horridus 4

Pygmephorus johnstoni 4

Pygmephorus lutterloughae 5

Pygmephorus mahunkai 5

Pygmephorus moreohorridus

Pygmephorus nidicolus 5

Pygmephorus whitakeri 5

Pygmephorus spp. 5

Radfordia ewingi 4

Radfordia hylandi

see Dermacarus hylandi 1

Radfordia lemnina 4

Radfordia subuliger $\quad 4$

Radfordia spp. $\quad 4$

Rectofrontia fraterna see Rhadinopsylla fraterna $\quad 37$

Rectofrontia sectilis see Rhadinopsylla sectilis goodi

Rhadinopsylla arborea $\quad 37$

Rhadinopsylla difficilis $\quad 37$

Rhadinopsylla fraterna $\quad 37$

Rhadinopsylla linta $\quad 37$

Rhadinopsylla media 37

Rhadinopsylla rauschi $\quad 37$

Rhadinopsylla sectilis goodi

Rhadinopsylla sectilis sectilis $\quad 37$

Rhinolophopsylla palposus see Myodopsylla palposa

Saphiopsylla bishopi see Atyphloceras bishopi

Sarcophagidae $\quad 15$

Sarcoptes scabiei 3

Sarcoptidae 3

Sarcoptidae gen. spp.

Siphonaptera 17

Siphunculata 42

Solenopotes ferrisi 43

Spinturnicidae 9

Spinturnix americanus 9

Spinturnix globosus 9

Spinturnix spp. 9

Stachiella kingi 16

Steatonyssus occidentalis 9

Stenistomera alpina 38

Stenoponia americana $\quad 38$
Streblidae $\quad 15$

Tamiophila grandis 38

Tarsopsylla coloradensis see Tarsopsylla octodecimdentata coloradensis 28

Tarsopsylla octodecimdentata coloradensis 28

Tetragonyssus microti see Laelaps kochi $\quad 8$

Thrassis acamantis $\quad 28$

Thrassis bacchi bacchi 29

Thrassis gladiolis gladiolis see Thrassis bacchi bacchi

29

Thrassis petiolatus 29

Thrassis spenceri spenceri 29

Thrassis spenceri vancouverensis see Thrassis spenceri spenceri

Trichobius spp. $\quad 15$

Trichodectes canis 16

Trichodectes ermineae $\quad 16$

Trichodectes kingi see Stachiella kingi 16

Trichodectes minutus $\quad 16$

Trichodectes octomaculatus

Trichodectes osborni $\quad 16$

Trichodectes parallelus $\quad 16$

Trichodectes pinguis euarctidos

Trichodectes tibialis $\quad 16$

Trichodectidae 16

Trichoecius tenax 3

Trichoecius spp. 3

Tricholipeurus lipeuroides $\quad 16$

Tricholipeurus parallelus $\quad 17$

Tricholipeurus virginianus see Tricholipeurus lipeuroides $\quad 16$

Tricholipeurus spp.

Trichopsylla abantis see Megabothris abantis 19

Trichopsylla acerba see Megabothris acerbus $\quad 19$

Trichopsylla asio asio see Megabothris asio asio $\quad 20$

Trichopsylla eumolpi eumolpi see Monopsyllus eumolpi eumolpi

Trichopsylla lotoris see Chaetopsylla lotoris

Trichopsylla quirini see Megabothris quirini

Trichopsylla setosus see Chaetopsylla setosa

Trichopsylla ursi see Chaetopsylla tuberculaticeps

Trichopsylla vison see Monopsyllus vison $\quad 23$

Trichopsylloides oregonensis 38

Trombicula jamesoni 6

Trombicula spp. 6

Trombiculidae 5

Trombiculidae gen. spp. 6

Trombidiidae 6

Typhlopsylla charlottensis see Catallagia charlottensis 
Typhlopsylla grandis see Tamiophila grandis

Uniramia 13

Vermipsyllidae

Wohlfahrtia meigenii see Wohlfahrtia vigil
Wohlfahrtia vigil $\quad 15$

Xenopsylla cheopis 41

Xenoryctes latiporus 1

Xenoryctes nudus 1

Zumptiella bakeri 6

\section{HOST INDEX}

Alces alces $\quad 44$

Alces alces americana $\quad 44$

Alces americanus

see Alces alces americana $\quad 44$

Alopex lagopus $\quad 36$

Alopex lagopus innuitus see Alopex lagopus lagopus

46

Alopex lagopus lagopus $\quad 46$

Antilocapra americana americana

Antilocapridae $\quad 44$

Aplodontia pacifica see Aplodontia rufa pacifica

61

Aplodontia rufa 61

Aplodontia rufa pacifica

Aplodontia rufa rufa 61

Aplodontia spp. $\quad 61$

Aplodontidae 61

Artiodactyla $\quad 44$

Bison bison 44

Blarina brevicauda 53

Blarina brevicauda angusta $\quad 54$

Blarina brevicauda manitobensis

Blarina brevicauda pallida 54

Blarina brevicauda talpoides 54

Blarina spp. 54

Bovidae 44

Canidae $\quad 46$

Canidae undetermined spp. $\quad 46$

Canis latrans $\quad 46$

Canis latrans incolatus $\quad 46$

Canis latrans lestes

46

Canis lupus 46

Canis lupus columbianus $\quad 46$

Canis spp. $\quad 46$

Carnivora 46

Castor canadensis

Castoridae 61

Castoridae undetermined spp.

Cavidae 61

Cervidae $\quad 44$

Cervidae undetermined spp. $\quad 45$

Cervus canadensis see Cervus elaphus canadensis

Cervus canadensis nelsoni see Cervus elaphus nelsoni $\quad 44$

Cervus elaphus 44

Cervus elaphus canadensis $\quad 44$

Cervus elaphus nelsoni $\quad 44$

Chiroptera 52

Citellus columbianus columbianus see Spermophilus columbianus

columbianus $\quad 82$

Citellus franklinii

see Spermophilus franklinii

82

Citellus richardsonii

see Spermophilus richardsonii

83

Citellus richardsonii richardsonii

see Spermophilus richardsonii

richardsonii 83

Citellus spp.

see Spermophilus spp.

Citellus tridecemlineatus

see Spermophilus tridecemlineatus

83

Citellus tridecemlineatus tridecemlineatus see Spermophilus tridecemlineatus tridecemlineatus 83

Clethrionomys dawsoni see Clethrionomys rutilus dawsoni

Clethrionomys dawsoni dawsoni see Clethrionomys rutilus dawsoni

Clethrionomys gapperi 62

Clethrionomys gapperi athabascae

Clethrionomys gapperi cascadensis

Clethrionomys gapperi caurinus

Clethrionomys gapperi gapperi 63

Clethrionomys gapperi gaspeanus

Clethrionomys gapperi loringi 63

Clethrionomys gapperi ochraceus $\quad 64$

Clethrionomys gapperi pallescens $\quad 64$

Clethrionomys gapperi proteus $\quad 64$

Clethrionomys gapperi saturatus $\quad 64$

Clethrionomys gapperi ungava $\quad 64$

Clethrionomys rutilus $\quad 64$

Clethrionomys rutilus dawsoni $\quad 64$

Clethrionomys spp. $\quad 65$

Condylura cristata 57

Condylura cristata cristata $\quad 57$

Condylura cristata nigra $\quad 57$

Cynomys ludovicianus $\quad 78$

Cynomys ludovicianus ludovicianus

Dicrostonyx groenlandicus 65

Dicrostonyx groenlandicus kilangmiutuk 65

Dicrostonyx groenlandicus richardsoni 65

Dicrostonyx hudsonius 65

Dicrostonyx spp. 65

Dicrostonyx torquatus richardsoni see Dicrostonyx groenlandicus richardsoni 65

Dipodomys ordii $\quad 62$

Dipodomys ordii terrasus

Eptesicus fuscus $\quad 52$ 
Eptesicus fuscus fuscus 52

Eptesicus fuscus pallidus 52

Erethizon dorsatum 61

Erethizon dorsatum dorsatum

Erethizon dorsatum epixanthium

Erethizon dorsatum nigrescens

Erethizon sp. 61

Erethizontidae 61

Eumops perotis californicus

52

Eutamias amoenus $\quad 78$

Eutamias amoenus affinis 79

Eutamias amoenus felix $\quad 79$

Eutamias amoenus ludibondus

Eutamias amoenus luteiventris

Eutamias borealis

see Eutamias minimus borealis

Eutamias minimus 79

Eutamias minimus borealis 79

Eutamias minimus minimus $\quad 79$

Eutamias minimus selkirki 79

Eutamias spp. $\quad 79$

Eutamias townsendii 79

Eutamias townsendii cooperi

Evotomys gapperi

see Clethrionomys gapperi

Evotomys gapperi saturatus

see Clethrionomys gapperi saturatus

64

Evotomys saturatus

see Clethrionomys gapperi $\quad 62$

Evotomys ungava

see Clethrionomys gapperi ungava

64

Felidae $\quad 47$

Geomyidae 61

Geomys sp. $\quad 61$

Glaucomys sabrinus $\quad 80$

Glaucomys sabrinus alpinus $\quad 80$

Glaucomys sabrinus canescens $\quad 80$

Glaucomys sabrinus columbiensis $\quad 80$

Glaucomys sabrinus fuliginosus $\quad 80$

Glaucomys sabrinus gouldi $\quad 80$

Glaucomys sabrinus macrotis $\quad 80$

Glaucomys sabrinus makkovikensis

Glaucomys sabrinus oregonensis

Glaucomys spp. $\quad 80$

Glaucomys volans $\quad 80$

Glaucomys volans volans

Gulo gulo see Gulo luscus $\quad 47$

Gulo gulo luscus see Gulo luscus $\quad 47$

Gulo luscus $\quad 47$

Heteromyidae 62

Insectivora 53

Lagomorpha 58

Lagurus curtatus 66

Lasionycteris noctivagans

Lemmus sibiricus $\quad 66$

Lemmus sibiricus trimucronatus

66

Lemmus spp. $\quad 66$

Lemmus trimucronatus

see Lemmus sibiricus

66
Lemmus trimucronatus trimucronatus see Lemmus sibiricus trimucronatus

Leporidae $\quad 58$

Leporidae undetermined spp.

59

Lepus americanus $\quad 58$

Lepus americanus bairdii $\quad 58$

Lepus americanus columbiensis

Lepus americanus dalli 58

Lepus americanus macfarlani see Lepus americanus dalli

Lepus americanus struthopus

Lepus americanus virginianus

Lepus arcticus $\quad 59$

Lepus arcticus arcticus $\quad 59$

Lepus campestris

see Lepus townsendii campanius 59

Lepus capensis $\quad 59$

Lepus capensis hybridus

Lepus europeaus see Lepus capensis

Lepus glacialis see Lepus arcticus arcticus

Lepus spp. 59

Lepus townsendii $\quad 59$

Lepus townsendii campanius

Lynx baileyi see Lynx rufus baileyi

Lynx canadensis $\quad 47$

Lynx canadensis canadensis

Lynx fasciatus see Lynx rufus fasciatus

Lynx rufus 47

Lynx rufus baileyi

Lynx rufus fasciatus 47

Lynx spp. $\quad 47$

Mammalia $\quad 44$

Mammalia undetermined spp.

Marmota caligata

81

Marmota caligata cascadensis

Marmota caligata okanagana

Marmota flaviventris

81

Marmota flaviventris avara

Marmota monax 81

Marmota monax canadensis

Marmota monax petrensis

Marmota monax rufescens

81

Marmota spp. 81

Marmota vancouverensis

Martes actuosa

see Martes americana actuosa

Martes americana 47

Martes americana abietinoides

Martes americana actuosa

Martes americana americana

Martes americana caurina

Martes pennanti 48

Martes pennanti pennanti

48

Mephitis mephitis 48

Mephitis mephitis mephitis

Mephitis spp. $\quad 48$

Microsorex hoyi 54

Microsorex hoyi hoyi 55 
Microsorex spp. $\quad 55$

Microtus chrotorrhinus 66

Microtus chrotorrhinus chrotorrhinus 66

Microtus drummondii

see Microtus pennsylvanicus drummondii 68

Microtus enixus

see Microtus pennsylvanicus enixus 69

Microtus longicaudus 66

Microtus longicaudus macrurus 66

Microtus longicaudus mordax

see Microtus longicaudus

66

Microtus longicaudus vellerosus

Microtus minor

see Microtus ochrogaster minor

Microtus miurus 67

Microtus montanus canascens 67

Microtus nanus canascens

see Microtus montanus canascens

Microtus ochrogaster $\quad 67$

Microtus ochrogaster minor

Microtus oeconomus $\quad 67$

Microtus oeconomus macfarlani

Microtus oeconomus operarius

Microtus operarius

see Microtus oeconomus operarius

Microtus oregoni

67

Microtus oregoni serpens

67

Microtus pennsylvanicus

68

Microtus pennsylvanicus acadicus

Microtus pennsylvanicus drummondii

Microtus pennsylvanicus enixus - $\quad 69$

Microtus pennsylvanicus insperatus

Microtus pennsylvanicus labradorius

Microtus pennsylvanicus pennsylvanicus 69

Microtus pennsylvanicus terraenovae 69

Microtus pinetorum scalopsoides

Microtus richardsoni $\quad 69$

Microtus richardsoni richardsoni

Microtus serpens see Microtus oregoni serpens

Microtus spp. $\quad 70$

Microtus tetramerus see Microtus townsendii tetramerus

Microtus townsendii $\quad 69$

Microtus townsendii tetramerus

Microtus townsendii townsendii

Microtus vellerosus

see Microtus longicaudus vellerosus

Microtus xanthognathus $\quad 70$

Molossidae 52

Muridae 62

Muridae undetermined spp.

Mus musculus $\quad 70$

Mus musculus musculus

see Mus musculus

Mus spp. $\quad 70$

Mustela americana see Martes americana

Mustela americana actuosa see Martes americana actuosa

Mustela arctica see Mustela erminea
Mustela arizonensis see Mustela frenata arizonensis

Mustela cicognanii cicognanii see Mustela erminea cicognanii

Mustela cicognanii richardsonii see Mustela erminea richardsonii

Mustela erminea 48

Mustela erminea anguinae $\quad 48$

Mustela erminea arctica 48

Mustela erminea bangsi 48

Mustela erminea cicognanii 49

Mustela erminea fallenda 49

Mustela erminea invicta 49

Mustela erminea richardsonii

Mustela frenata 49

Mustela frenata arizonensis

Mustela frenata longicauda

Mustela frenata nevadensis $\quad 49$

Mustela frenata novaboracensis

Mustela frenata oribasus $\quad 50$

Mustela longicauda see Mustela frenata longicauda

Mustela nivalis rixosa $\quad 50$

Mustela putorius $\quad 50$

Mustela rixosa see Mustela nivalis rixosa

Mustela rixosa rixosa see Mustela nivalis rixosa

Mustela spp. $\quad 50$

Mustela vison 50

Mustela vison energumenos

Mustela vison vison $\quad 50$

Mustelidae 47

Mustelidae undetermined spp.

Myotis keenii septentrionalis $\quad 52$

Myotis lucifugus 53

Myotis lucifugus alascensis $\quad 53$

Myotis lucifugus lucifugus 53

Myotis lucifugus pernox $\quad 53$

Myotis spp. $\quad 53$

Myotis yumanensis saturatus

Napaeozapus insignis $\quad 87$

Napaeozapus insignis frutectanus

Napaeozapus insignis insignis 88

Napaeozapus spp. $\quad 88$

Neotoma cinerea $\quad 70$

Neotoma cinerea cinerea 71

Neotoma cinerea drummondii 71

Neotoma cinerea occidentalis $\quad 71$

Neotoma cinerea saxamans see Neotoma cinerea occidentalis

Neotoma spp. $\quad 71$

Neurotrichus gibbsii 57

Neurotrichus gibbsii gibbsii

Neurotrichus spp. $\quad 57$

Ochotona collaris $\quad 60$

Ochotona princeps $\quad 60$

Ochotona princeps brooksi $\quad 60$

Ochotona princeps brunnescens

Ochotona princeps cuppes $\quad 60$

Ochotona princeps fenisex $\quad 60$

Ochotona princeps princeps $\quad 60$
49

88

1 
Ochotona spp. $\quad 60$

Ochotonidae $\quad 60$

Odocoileus hemionus

44

Odocoileus hemionus columbianus

Odocoileus hemionus hemionus

Odocoileus spp. $\quad 45$

Odocoileus virginianus 45

Odocoileus virginianus borealis

Ondatra spp. $\quad 71$ 71

Ondatra zibethicus

Ondatra zibithicus osoyoosensis

Ondatra zibethicus zibethicus 71

Onychomys leucogaster 71

Onychomys leucogaster missouriensis

Oreamnos americanus 44

Oreamnos americanus missoulae

Ovis canadensis 44

Ovis canadensis californiana

Ovis canadensis canadensis

Parascalops breweri $\quad 57$

Parascalops spp. $\quad 58$

Pedomys ochrogaster see Microtus ochrogaster

Perognathus lordi lordi see Perognathus parvus lordi

Perognathus parvus laingi $\quad 62$

Perognathus parvus lordi $\quad 62$

Peromyscus arcticus see Peromyscus maniculatus borealis

Peromyscus canadensis see Peromyscus maniculatus $\quad 72$

Peromyscus leucopus 71

Peromyscus leucopus caudatus $\quad 72$

Peromyscus leucopus noveboracensis

Peromyscus maniculatus 72

Peromyscus maniculatus abietorum 73

Peromyscus maniculatus algidus 73

Peromyscus maniculatus alpinus 73

Peromyscus maniculatus anticostiensis

Peromyscus maniculatus artemisiae 73

Peromyscus maniculatus austerus $\quad 73$

Peromyscus maniculatus bairdii 73

Peromyscus maniculatus borealis $\quad 74$

Peromyscus maniculatus gracilis $\quad 74$

Peromyscus maniculatus interdictus

Peromyscus maniculatus keeni 74

Peromyscus maniculatus macrorhinus

Peromyscus maniculatus maniculatus

Peromyscus maniculatus nebrascensis

Peromyscus maniculatus oreas $\quad 74$

Peromyscus maniculatus osgoodi see Peromyscus maniculatus nebrascensis 74

Peromyscus maniculatus prevostensis $\quad 74$

Peromyscus sitkensis $\quad 74$

Peromyscus sitkensis prevostensis see Peromyscus maniculatus prevostensis 74

Peromyscus spp. 75

Phenacomys intermedius $\quad 75$

Phenacomys intermedius celatus $\quad 76$

Phenacomys intermedius intermedius $\quad 76$

Phenacomys spp. $\quad 76$

Phenacomys ungava see Phenacomys intermedius celatus

Phenacomys ungava ungava see Phenacomys intermedius celatus

Pitymys pinetorum scalopsoides see Microtus pinetorum scalopsoides

Procyon lotor 52

Procyon lotor vancouverensis 52

Procyon spp. 52

Procyonidae 52

Putorius cicognanii see Mustela erminea cicognanii

Putorius richardsonii see Mustela erminea richardsonii

Rangifer caribou sylvestris see Rangifer tarandus caribou

Rangifer montanus see Rangifer tarandus caribou

Rangifer tarandus $\quad 45$

Rangifer tarandus caribou $\quad 45$

Rangifer tarandus groenlandicus

Rattus norvegicus 76

Rattus rattus 76

Rattus rattus alexandrinus $\quad 76$

Rattus rattus norvegicus see Rattus norvegicus

Rattus rattus rattus 76

Rattus spp. $\quad 76$

Rodentia 61

Rodentia undetermined spp.

Scalopus spp. $\quad 58$

Scapanus aquaticus $\quad 58$

Scapanus latimanus $\quad 58$

Scapanus orarius $\quad 58$

Scapanus orarius orarius

Scapanus orarius schefferi 58

Scapanus spp. $\quad 58$

Scapanus townsendii $\quad 58$

Sciuridae $\quad 78$

Sciuridae undetermined spp. $\quad 86$

Sciuropterus spp. see Glaucomys spp. $\quad 80$

Sciurus carolinensis 81

Sciurus carolinensis leucotis see Sciurus carolinensis $\quad 81$

Sciurus carolinensis pennsylvanicus

Sciurus hudsonicus see Tamiasciurus hudsonicus

Sciurus hudsonicus richardsoni see Tamiasciurus hudsonicus richardsoni 85

Sciurus niger $\quad 82$

Sciurus richardsoni baileyi see Tamiasciurus hudsonicus baileyi

Sciurus spp. $\quad 82$

Sorex arcticus $\quad 55$

Sorex arcticus arcticus

Sorex arcticus laricorum

55

Sorex cinereus 55

Sorex cinereus acadicus

Sorex cinereus cinereus

Sorex cinereus haydeni

55

Sorex cinereus miscix 
Sorex fumeus 56

Sorex gaspensis 56

Sorex obscurus

see Sorex vagrans obscurus

Sorex obscurus obscurus

see Sorex vagrans 56

Sorex obscurus setosus see Sorex vagrans 56

Sorex palustris 56

Sorex richardsonii see Sorex arcticus arcticus

Sorex spp. 57

Sorex trowbridgii 56

Sorex trowbridgii trowbridgii 56

Sorex vagrans 56

Sorex vagrans obscurus $\quad 56$

Sorex vagrans vagrans $\quad 56$

Sorex vagrans vancouverensis

Soricidae 53

Soricidae undetermined spp.

Spermophilus columbianus $\quad 82$

Spermophilus columbianus columbianus

82

Spermophilus franklinii $\quad 82$

Spermophilus lateralis $\quad 82$

Spermophilus lateralis tescorum

Spermophilus parryii $\quad 82$

Spermophilus parryii parryii

Spermophilus parryii plesius

Spermophilus richardsonii

Spermophilus richardsonii richardsonii 83

Spermophilus saturatus 83

Spermophilus spp. $\quad 83$

Spermophilus tridecemlineatus

Spermophilus tridecemlineatus

tridecemlineatus 83

Spermophilus undulatus see Spermophilus parryii $\quad 82$

Spermophilus undulatus parryii see Spermophilus parryii $\quad 82$

Spermophilus undulatus plesius see Spermophilus parryii plesius

Spilogale gracilis latifrons see Spilogale putorius gracilis

Spilogale gracilis olympica see Spilogale putorius gracilis

Spilogale phenax see Spilogale putorius phenax

Spilogale putorius $\quad 50$

Spilogale putorius gracilis $\quad 50$

Spilogale putorius latifrons 51

Spilogale putorius phenax 51

Spilogale spp. 51

Sylvilagus floridanus $\quad 59$

Sylvilagus floridanus mearnsii

Sylvilagus nuttalii $\quad 59$

Sylvilagus nuttalii grangeri

Sylvilagus nuttalii nuttalii

Sylvilagus spp. $\quad 59$

Synaptomys borealis $\quad 76$

Synaptomys borealis borealis

Synaptomys borealis chapmani

Synaptomys borealis medioximus
Synaptomys borealis truei $\quad 77$

Synaptomys borealis wrangeli see Synaptomys borealis truei

Synaptomys cooperi 77

Synaptomys cooperi cooperi $\quad 77$

Synaptomys spp. $\quad 77$

Talpidae $\quad 57$

Talpidae undetermined spp. $\quad 58$

Tamiasciurus douglasii $\quad 84$

Tamiasciurus douglasii cascadensis see Tamiasciurus douglasii mollipilosus 84

Tamiasciurus douglasii mollipilosus $\quad 84$

Tamiasciurus hudsonicus $\quad 84$

Tamiasciurus hudsonicus baileyi 84

Tamiasciurus hudsonicus columbiensis 85

Tamiasciurus hudsonicus douglasii see Tamiasciurus douglasii $\quad 84$

Tamiasciurus hudsonicus gymnicus

Tamiasciurus hudsonicus hudsonicus

Tamiasciurus hudsonicus lanuginosus

Tamiasciurus hudsonicus laurentianus $\quad 85$

Tamiasciurus hudsonicus loquax 85

Tamiasciurus hudsonicus minnesota

Tamiasciurus hudsonicus picatus

Tamiasciurus hudsonicus preblei $\quad 85$

Tamiasciurus hudsonicus richardsoni

Tamiasciurus hudsonicus streatori

Tamiasciurus hudsonicus ungavensis

Tamiasciurus spp. $\quad 86$

Tamias spp. $\quad 86$

Tamias striatus $\quad 86$

Tamias striatus griseus $\quad 86$

Tamias striatus lysteri $\quad 86$

Taxidea taxus 51

Taxidea taxus taxus 61

Thomomys fuscus see Thomomys talpoides

Thomomys fuscus fuscus see Thomomys talpoides

Thomomys spp. 62

Thomomys talpoides 61

Thomomys talpoides andersoni

Thomomys talpoides fuscus

$62^{62}$

Thomomys talpoides incensus

Ursidae $\quad 52$

Ursidae undetermined spp. $\quad 52$

Ursus americanus $\quad 52$

Ursus americanus americanus $\quad 52$

Ursus americanus cinnamomum 52

Ursus arctos $\quad 52$

Ursus horribilis see Ursus arctos

52

Ursus spp. $\quad 52$

Vespertilio fuscus see Eptesicus fuscus

Vespertilionidae 52

Vulpes fulva rubricosa see Vulpes vulpes rubricosa

Vulpes spp. $\quad 46$

Vulpes vulpes $\quad 46$ 
Zapodidae $\quad 87$

Zapus hudsonius $\quad 88$

Zapus hudsonius acadicus

Zapus hudsonius canadensis 88

Zapus hudsonius ontarioensis see Zapus hudsonius canadensis 88 Zapus princeps $\quad 88$

Zapus princeps idahoensis Zapus princeps minor

Zapus spp. $\quad 88$

Zapus trinotatus trinotatus

88 

